\title{
Review of Metastable States in Heavy Nuclei
}

\section{G. D. Dracoulis *}

Department of Nuclear Physics, R.S.P.E. Australian National University, Canberra, A.C.T. 0200, Australia

\section{P. M. Walker}

Department of Physics, University of Surrey, Guildford, Surrey GU2 7XH, United Kingdom

E-mail: P.Walker@Surrey.ac.uk

\section{F. G. Kondev}

Nuclear Engineering Division, Argonne National Laboratory, Argonne, Illinois, 60439, USA

E-mail: kondev@anl.gov

\begin{abstract}
.
The structure of nuclear isomeric states is reviewed in the context of their role in contemporary nuclear physics research. Emphasis is given to high-spin isomers in heavy nuclei, with $A \gtrsim 150$. The possibility to exploit isomers to study some of the most exotic nuclei is a recurring theme. In spherical nuclei, the role of octupole collectivity is discussed in detail, while in deformed nuclei the limitations of the $K$ quantum number are addressed. Isomer targets and isomer beams are considered, along with applications related to energy storage, astrophysics, medicine, and experimental advances.
\end{abstract}

\footnotetext{
* deceased
} 


\section{Contents}

1 Introduction $\quad 3$

1.1 History . . . . . . . . . . . . . . . . . . . . . . . . 3

1.2 Imperatives . . . . . . . . . . . . . . . . . . . . 4

1.3 Scope ................................. 6

2 Background to isomer formation $\quad 7$

2.1 Transition strengths and lifetimes . . . . . . . . . . . . . . 9

2.2 Seniority isomers in spherical nuclei . . . . . . . . . . . . . . . 10

$2.3 K$-isomers in deformed nuclei . . . . . . . . . . . . . . . . . . 12

$2.4 \quad K$-hindered decays $\ldots \ldots \ldots \ldots$

3 Deformed and transitional nuclei with $A \approx 160-190 \quad 14$

3.1 Multi-quasiparticle excitations and common building blocks . . . . . . . 14

3.2 Multi-quasiparticle calculations . . . . . . . . . . . . . 16

3.3 Multi-quasiparticle isomers in the mass-180 region . . . . . . . . . . . 19

$3.4 \quad K$-hindrances . . . . . . . . . . . . . . . . . . . . . . . 20

3.5 Static electromagnetic moments of multi-quasiparticle isomers . . . . . 24

$3.6 \quad A \sim 190$; transitional nuclei . . . . . . . . . . . . . . . . 25

3.6.1 Isomers and transition strengths in transitional nuclei . . . . . . 27

3.6.2 Predictions of low-lying high- $K$ intrinsic states . . . . . . . . 28

4 Spherical regions $\quad 30$

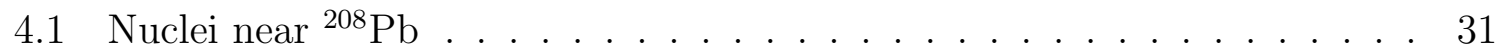

4.1.1 Residual interactions and the empirical shell model (ESM) . . . . 31

4.1.2 Octupole-vibration mixing . . . . . . . . . . . . . . . 33

4.1.3 Valence configurations: the example of ${ }^{212} \mathrm{Po} \ldots . . . . . . . .33$

4.1.4 Valence particles for $N \geq 126$ and predicted isomers in the $N=$ 128 chain . . . . . . . . . . . . . . . . . . 34

4.1.5 Isomerism and shape coexistence in the neutron-deficient $\mathrm{Pb}$ region 37

4.1.6 Particle and hole nuclei neighbouring $Z=82 \ldots \ldots$. . . . . . 37

4.1.7 Neutron-rich $\mathrm{Hg}$ and $\mathrm{Pb}$ cases . . . . . . . . . . . . . . . . 38

4.1.8 Multiparticle core-excited isomers: ${ }^{212} \mathrm{Rn}$ and the radon region . 39

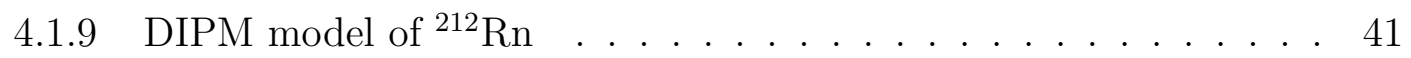

4.1 .10 Multi-particle isomers and blocking . . . . . . . . . . . . . 43

4.2 Nuclei near ${ }^{146} \mathrm{Gd} \quad \ldots \ldots \ldots$. . . . . . . . . . . . . . . 43

5 Deformed nuclei with $A>190$ : extreme shapes, fission and $K$ hindrance

5.1 Second-well fission isomers f . . . . . . . . . . . . . . . . . 47

5.2 Superdeformed bandheads . . . . . . . . . . . . . . . . . . . . 48

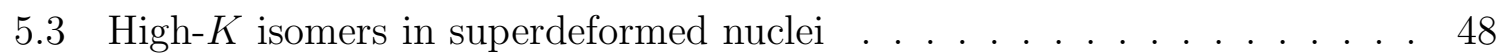


5.4 High- $K$ isomers in superheavy nuclei . . . . . . . . . . . . . . . . 49

5.4.1 Predictions for $Z=100-110 \ldots \ldots \ldots$. . . . . . 50

$5.4 .2 K$-hindrance in superheavy nuclei . . . . . . . . . . . 52

$6 \quad$ Isomer targets $\quad 52$

$\begin{array}{lll}7 & \text { Isomer beams } & 53\end{array}$

8 Energy traps, induced deexcitation, lasers and astrophysics $\quad 54$

$8.1{ }^{180} \mathrm{Ta} \ldots \ldots \ldots \ldots \ldots \ldots$

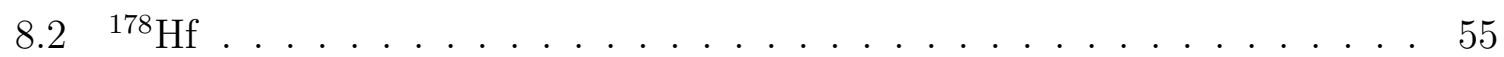

8.3 NEEC and NEET . . . . . . . . . . . . . . . . . . . 56

8.4 Radioactive beams . . . . . . . . . . . . . . . . . . . 57

8.5 Isomers and nucleosynthesis . . . . . . . . . . . . . . . 58

9 Isomers for medicine $\quad 59$

10 Key experimental developments $\quad 60$

10.1 Neutron-deficient and very heavy nuclei . . . . . . . . . . . . . . . 60

10.1.1 Recoil-decay tagging and related techniques . . . . . . . . . . 60

10.2 Neutron-rich nuclei . . . . . . . . . . . . . . . . . . . . 62

10.2.1 Relativistic projectile fragmentation . . . . . . . . . . . . 62

10.2.2 Incomplete fusion reactions . . . . . . . . . . . . . 63

10.2.3 Fusion-evaporation reactions with radioactive beams . . . . . . . 63

10.2.4 Multi-nucleon transfer and deep-inelastic reactions . . . . . . . . 64

10.2.5 Isomers in storage rings . . . . . . . . . . . . 66

10.3 Sub-nanosecond timing detectors: $\mathrm{LaBr}_{3} \ldots \ldots \ldots$. . . . . . . . . 68

10.4 Separating isomers from ground states . . . . . . . . . . . . . . . . . . . . . . . 69

10.4.1 Mass selection . . . . . . . . . . . . . . . . 69

10.4.2 Hyperfine structure . . . . . . . . . . . . . . . . 69

$\begin{array}{ll}11 \text { Open questions and closing comments } & 70\end{array}$

12 References $\quad 71$

\section{Introduction}

\subsection{History}

The discovery of nuclear isomerism is credited to the work of Otto Hahn in 1921 [1] although the term isomer in the nuclear context probably came from Frederick Soddy who applied it to nuclei, in analogy with chemical isomers, to describe long-lived nuclear states with nuclear properties substantially different from others formed by the same number of protons and neutrons [2]. At a time when all nuclear states were anticipated to 
have lifetimes less than $10^{-13} \mathrm{~s}$, there was considerable speculation as to their origin (see for example Ref. [3]). A crucial theoretical insight came in 1936 from von Weizsäcker [4], who showed that the combination of a large angular momentum change and low transition energy could lead to a long half-life for electromagnetic decay.

The early review by Segrè and Helmholz [5] attempted to quantify von Weizsäcker's idea and by the early 1950's, the discovery of isomers in many nuclei and their interpretation $[6,7,8]$ were playing a pivotal role in the development of models describing spherical and deformed nuclei. In spherical odd-A nuclei the shell model with spin-orbit splitting predicted that orbitals with significant differences in orbital angular momentum would be present at the Fermi surface, giving close-lying low-energy states with very different quantum numbers. The probabilities for the electromagnetic transitions connecting such states were likely to be low, hence isomers could be expected. Their observation and classification was a direct test of the properties of the orbitals present, and the purity of the configurations, and therefore the nuclear potential: a snapshot, essentially, of the important intrinsic states in nuclei.

In parallel, evidence for the existence, and contributions to the understanding, of the characteristics of deformed nuclei evolved: the seminal 1953 paper of Bohr and Mottelson [9] interpreted the $\gamma$-ray cascade following the decay of an unusual 5.5 hour isomer [10] in ${ }^{180} \mathrm{Hf}$ as a sequence of transitions within the rotational band of an axially-symmetric deformed nucleus. Surprisingly, the moment-of-inertia deduced from the state energyspacing was only about a third of that expected for rigid rotation of a nucleus with the deformation implied by the quadrupole moments [11, 12], a fact subsequently attributed to pairing-induced superfluidity in nuclei [13]. The long half-life turned out not to be due to a large angular momentum change but to the breaking of a (new) selection rule related to conservation of $K$, the projection of the nuclear total angular momentum on the deformation axis. This terminology came into currency in 1955 [14], with robust selection rules. Discovery of the high- $K$ isomer in ${ }^{180} \mathrm{Hf}$ was clearly a trigger for a number of major steps in the understanding of collective nuclear structure, as highlighted by Aage Bohr in his Nobel prize lecture of 1975 [15].

The high-spin studies of the late 70s and early 80 s resulted in the discovery of many isomeric, multiparticle states in several regions, formed by aligning particles in the equatorial plane of spherical nuclei or the belt of deformed nuclei. The excitement this generated was reflected in the statement recorded by Bohr and Mottelson in the Nobel symposium of 1980: The presence of many quasiparticles with aligned angular momenta presents us with nuclear matter under fascinating new conditions, representing a novel type of symmetry breaking [16]. One question that has yet to be answered perhaps, is how many particles can be aligned before the concept of a mean-field is disrupted.

\subsection{Imperatives}

From the theoretical point-of-view the terms "isomer" and "isomeric" are used generally where different characteristics or configurations arising from the same constituents will 
result in a reduced overlap between initial and final state wave functions, and hence significant inhibition for transitions between them - as with shape isomers, for example.

On the technical side, any state which has a directly measurable lifetime, in the sense of an electronic measurement, even down to the sub-nanosecond regime, could be termed an isomer, or more properly a metastable state. The semantics are less important than the consideration that the states are relatively long-lived compared to normal states in a particular nucleus or nuclear regime [17]. In other words, they are metastable. There is an additional distinction to be made between intrinsic states and collective states that might, coincidentally, have directly measurable lifetimes. As well as the nuclear structure considerations, lifetimes of the order of a few nanoseconds or longer provide an experimental tool which facilitates a variety of techniques: accurate measurement of transition strengths, time-differential measurement of nuclear moments [18], unambiguous placement of transitions in nuclear level schemes through temporal ordering, and substantially improved sensitivity to weakly populated states. Indeed, it can be the case that it is states populated in an isomer's decay that are of primary interest, rather than the isomer itself. The sensitivity is gained because otherwise complex spectra can be dramatically simplified by separation into different time regimes, either electronically, physically in space, or both. The time correlations allow precise identification of transitions following and preceding high-lying isomers, opening up the prospect of unambiguous placement and the ability to bootstrap to otherwise inaccessible regions of high excitation energy and spin.

The idea that the lifetime of a nuclear state decaying by a single transition depends on the transition energy, $\Delta E$, and the change in quantum numbers between the isomer and the state to which it decays translates to the relation

$$
\tau \propto|<f| T_{\lambda}|i>|^{2} /(\Delta E)^{2 \lambda+1},
$$

where $\lambda$ is the multipole order and $T_{\lambda}$ is the transition operator between the initial, $i$, and final, $f$, states. This depends on three factors, the energy and multipole order (combined) and the underlying transition matrix element, which itself may be the product of a number of factors. If $\Delta E$ is small and the multipole order is large, the lifetime is likely to be relatively long. This can occur by chance, in the sense of a random energy spacing, or because the configuration of the upper state represents an unusually efficient way for the nucleus to carry angular momentum (a nuclear structure effect), hence its relatively low energy and high spin. Alternatively, a long lifetime might signal a distinct change in nuclear structure, resulting in a small overlap and therefore a small matrix element. This could indicate a large configuration change (the rearrangement of many orbits) or a large shape change, or even a major re-orientation of a spin vector, any one of which will lead to inhibited transitions. The underlying factor here is that different modes of excitation are not confined to particular regions of the nuclear chart but can develop within a single nucleus, since the excitations themselves modify the nuclear mean-field.

Studies of isomers aimed at elucidating nuclear structure therefore exploit the 
related prospects for:

- making new discoveries with high sensitivity

- characterising unusual nuclear structures and configurations

- probing both mean-field potentials and dynamics

- classifying shapes - spherical, deformed and coexisting

- exposing aspects of wave-function hybridisation, such as configuration mixing and $K$-mixing.

Other imperatives include the possibility of applications stemming from the serendipitous discovery of states with specific properties. These range from opportunities for the storage and release of energy [17] and studies of coupled atomic-nuclear effects $[19,20,21,22]$, to applications in medicine (see for example [23, 24, 25]). The existence of isomers could well have a role in stellar processes $[26,27]$ while the existence of possible $K$-violating paths that could connect the low-lying ground and isomeric states in nuclides such as ${ }^{176} \mathrm{Lu}$ and ${ }^{180} \mathrm{Ta}$ via photo-excitation in a stellar environment, thus controlling their abundances, continues to challenge both theory and experiment $[26,28,29,30,31,32,33,34,35]$.

\subsection{Scope}

This review will begin with an outline of some of the nuclear-structure factors operative in the formation of isomers, such as seniority cancellation and $K$-forbiddenness (Sect. 2). Examples will be drawn from the "traditional" regions of isomerism, near the spherical and deformed shells. The high- $K$ isomers in well-deformed nuclei near mass-180 and also in the transitional nuclei near mass-190 will be covered in Sect. 3, while spherical regions and multi-particle shell-model isomers will be discussed in Sect. 4. These will include the trans-lead nuclei (Sect. 4.1) where coupling to octupole vibrations is important, and the mass-150 region where similar high-spin multi-particle configurations occur, but where shape changes may play a more significant role (Sect. 4.2).

Dynamic and static shape changes are related to the formation of competing minima in the nuclear potential that leads to shape coexistence. The high-spin superdeformation, that was discovered several decades ago, falls into this category. Low-spin superdeformation was already well-known in heavy nuclei to lead to fission isomers. Implications for the existence and properties of either bandhead or $K$-isomers in the second well will be covered in Sect. 5. The status of studies on superheavy nuclei will also be included in this section. These studies hark back to the role played earlier by isomers in identifying key orbitals near the Fermi surface (Sect. 5.4) with the present hope of discriminating between mean-field theories that aim to predict the existence and stability of superheavy nuclei.

The possibilities to exploit isomer targets and isomer beams are discussed in Sect. 6 and 7 , respectively. In parallel with these (predominantly) nuclear structure studies, the expanding role of isomers in inter-disciplinary research, including opportunities for direct 
applications such as induced deexcitation for energy release, or for probing subtle effects at the nuclear/atomic interface, will be outlined in Sect. 8, together with astrophysical applications. Medical applications are discussed in Sect. 9.

Selected additional aspects of experimental development will be covered in Sect. 10, including recoil methods for the study of neutron-deficient nuclei; the use of deepinelastic and fragmentation reactions, particularly for studies in neutron-rich nuclei, including direct mass measurements with storage rings; fast-timing with $\mathrm{LaBr}_{3}$ detectors; and isomer separation techniques.

Taken together, these topics constitute too broad a canvas to be covered in detail, but hopefully we can provide some of the signposts to developments of current interest, as well as prospects for the future. In this context, a detailed analysis and review of the structure of high-spin states including isomers was given by de Voigt et al. three decades ago [36]. More recent reviews of isomers include a study of deformed nuclei in the mass180 region [37], a comprehensive review of shape coexistence where isomers play a role [38], as well as various popular commentaries (see for example Refs. [17, 23, 39, 40]). An extensive data review covering the decay properties and configurations of high- $K$ isomers in deformed nuclei with $A>100$ has also recently been completed [41]. The present work draws both on those results and on the Nobel symposium report by Dracoulis [42]. In addition, theoretical aspects of high- $K$ isomers have been discussed recently by Walker and $\mathrm{Xu}$ [43]. On the purely experimental side, the recent compilation associated with the 2012 NUBASE evaluation of Audi et al. [44] includes excited isomeric states with halflives greater than $100 \mathrm{~ns}$, as well the nuclear ground states; isomers with half-lives greater than $10 \mathrm{~ns}$ are tabulated by Jain et al. [45]; and electromagnetic moments have been compiled by Stone [46]. Furthermore, data for isomers and their decay properties are regularly published in the journal Nuclear Data Sheets and are included in the nuclear structure databases Evaluated Nuclear Structure Data File (ENSDF) and Unevaluated Nuclear Data List (XUNDL) [47].

In figure 1, a prespective is given of the occurrence of isomers throughout the nuclear chart, with data from Ref. [44]. From this figure, it is clear that the occurrence of isomers is not random, with concentrations both at closed shells and away from closed shells. It is the underlying physics associated with this non-random distribution that forms the basis of this review.

A contemporary issue is to what extent isomers might, at the limits of nuclear binding, provide extra stability, in the sense that their half-lives can exceed those of their corresponding ground states. There is discussion of this aspect in Sect. 5 .

\section{Background to isomer formation}

While much is known of the nuclear structure that controls the excitation energies and decay rates of nuclear states, it is essentially impossible to predict precisely their likely occurrence, and especially their lifetimes. This is because small energy differences between the initial state and possible final states can have a large effect on transition 


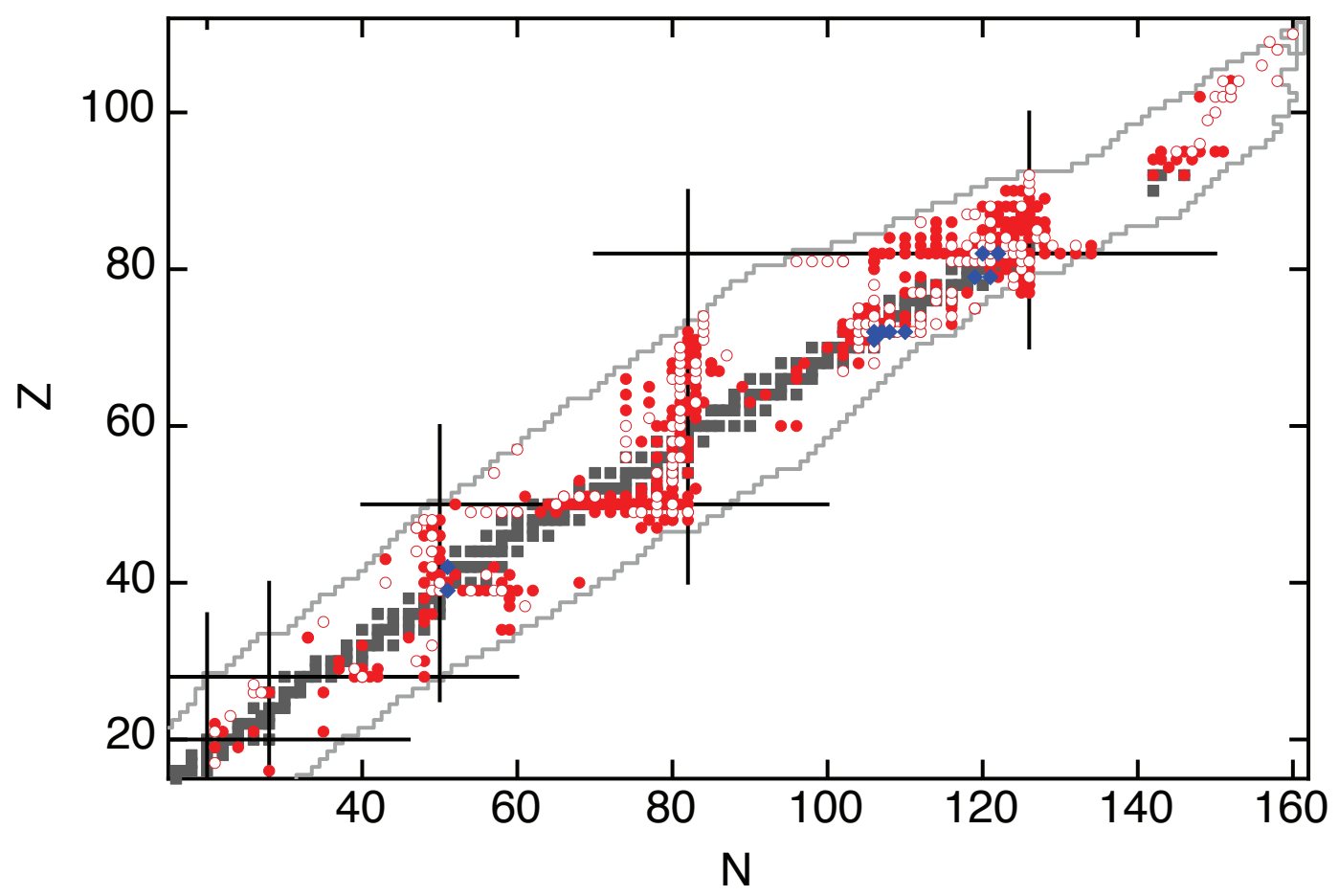

Figure 1. Nuclear chart illustrating the distribution of isomers with excitation energies greater than $600 \mathrm{keV}$, with data from Audi et al. [44]. The filled red circles correspond to $200 \mathrm{~ns}<T_{1 / 2}<100 \mu \mathrm{s}$, the open red circles correspond to $100 \mu \mathrm{s}$ $<T_{1 / 2}<1 \mathrm{hr}$, and the filled blue diamonds are for $T_{1 / 2}>1 \mathrm{hr}$.

rates. In the extreme case a small change in energy might result in the reversal of the disposition of close-lying states, so where a decay path may have been predicted, in reality, no path exists, or vice versa. Furthermore, since the transitions which do occur may be ones which are, in principle, forbidden, they must only occur through miniscule wave-function admixtures which are impossible to predict accurately. Hence, there is an inevitable reliance on phenomenology driven by experiment and discovery.

The traditional areas where such states are found, and indeed where more are expected, are in medium-heavy to heavy nuclei close to closed shells, where the valence particles can occupy orbits with high spin. This occurs near the closed neutron and proton shells with $N=82$ and $Z=50(A \approx 130), N=82, Z=64(A \approx 150)$, and $N=126, Z=82(A \approx 210)$, giving rise to multi-particle states with high-spin at relatively low energies. While these states have their origin in spherical configurations, coupling to the octupole vibration turns out to play a crucial role in their formation. In the well-deformed nuclei, which occur between closed shells, collective (rotational) states tend to dominate the yrast line $\ddagger$ except in the $A \approx 180$ region. There, high- $K$ multi-quasiparticle states, formed by summing the projections on the symmetry axis of a number of high-spin unpaired nucleons, are sufficiently low in energy to compete with $\ddagger$ the locus of the lowest energy states as a function of spin 
purely collective excitations.

\subsection{Transition strengths and lifetimes}

It is useful to classify transitions in terms of their strength with respect to nominal single-particle transitions, that is, those in which the orbit of one particle changes, under conditions which imply that there are no particular inhibitions due to changes in the quantum numbers etc. This corresponds to the Weisskopf unit (W.u.) for the various electric and magnetic radiations of different multipole order, detailed formulae for which can be found in textbooks. The half-life in nanoseconds equivalent to a Weisskopf unit is

$$
\begin{aligned}
& T_{1 / 2}(E 1)=6.76 \times A^{-2 / 3} E^{-3} \times 10^{-6} \\
& T_{1 / 2}(M 1)=2.20 \times E^{-3} \times 10^{-5} \\
& T_{1 / 2}(E 2)=9.52 \times A^{-4 / 3} E^{-5} \\
& T_{1 / 2}(M 2)=3.10 \times A^{-2 / 3} E^{-5} \times 10^{1} \\
& T_{1 / 2}(E 3)=2.04 \times A^{-2} E^{-7} \times 10^{7}
\end{aligned}
$$

where the transition energy $E$ is in $\mathrm{MeV}$ and $A$ is the atomic mass number [41].

Taking a mass number of $A=200$ and a transition energy of $200 \mathrm{keV}$, we can calculate half-lives for what would be typical decays in a given structure context, remembering that internal conversion will reduce the half-lives further. Electrical dipole (E1) transitions, for example, are normally retarded with respect to single-particle values by factors of $\sim 10^{-5}$ because of the absence of intrinsic nuclear orbitals which can be connected by the dipole operator so that

$$
T_{1 / 2}(E 1,200 \mathrm{keV}) \sim 2 \text { ns. }
$$

Electric quadrupole transitions in nuclei between single-particle or many-particle states have strengths which are less than the single-particle values (modified as will be discussed below by specific coupling effects) so that

$$
T_{1 / 2}(E 2,200 \mathrm{keV}) \gtrsim 25 \mathrm{~ns} .
$$

Collective E2 transitions, however, between members of rotational bands associated with deformed nuclei may be several hundred times faster, making their half-lives a small fraction of a nanosecond. Except in special cases, $M 1$ transitions are not usually associated with collective modes, and typically would have strengths of about 0.1 W.u., giving values similar to the collective $E 2$ case:

$$
T_{1 / 2}(M 1,200 \mathrm{keV}) \sim 0.03 \mathrm{~ns} .
$$

Magnetic quadrupole $(M 2)$ transitions are rarely stronger than a single-particle unit and then only between specific $j$-allowed single-particle orbits. Even those have longhalf-lives with

$$
T_{1 / 2}(M 2,200 \mathrm{keV}) \gtrsim 3 \mu \mathrm{s} .
$$


The lifetimes in general increase rapidly as the multipole order increases, so that singleparticle electric-octupole transitions of such a low energy are very slow, with

$$
T_{1 / 2}(E 3,200 \mathrm{keV}) \sim 40 \mathrm{~ms},
$$

and would be most unlikely to compete with other available decay paths. More importantly, E3 transitions associated with collective vibrations are a feature of some nuclei near closed shells and are 10-50 times stronger (see Sect. 4), hence the associated lifetimes are correspondingly shorter. Nevertheless, even for an enhancement of a factor of forty, a $200 \mathrm{keV} \mathrm{E3}$ transition would still result in a half-life of about a millisecond.

It should be clear from the above that isomers will occur even for "normal transitions". Nuclear structure effects which act to retard transitions further, or to lower the transition energies, will be superimposed on this.

\subsection{Seniority isomers in spherical nuclei}

As will be discussed later (Sect. 4), there are many isomers known in regions near closed shells, such as in the trans-lead nuclei. Most are spin traps [17] - low energy states that are forced to decay by high-multipole transitions - while others involve multiple changes in particle orbits resulting in significant inhibitions. Even at relatively low spins, however, most spherical even-even nuclei exhibit isomers, due not to orbital changes, but to wave-function cancellations.

Near the middle of specific shells, the $E 2$ transition strengths are reduced because of seniority cancellation $[48,49,50]$. For members of a $j^{n}$ multiplet in a single- $j$ shell, as a function of seniority, $s$, the reduced transition probability from spin $J$ to $J-2$ can be written:

$$
B(E 2 ; J \rightarrow(J-2)) \propto\left(\frac{\Omega-n}{\Omega-s}\right)^{2} \quad,
$$

where $n$ is the number of active particles and $2 \Omega=2 j+1$ is the shell degeneracy. This translates to a linear dependence of $\sqrt{B(E 2)}$ on $n$ and to a cancellation of the $B(E 2)$ strength in the middle of a shell. The situation in more complicated cases when the shells are not isolated is usually analysed in terms of quasiparticles by incorporating the particle and hole occupation probability factors $u$ and $v$ and an effective charge $\mathrm{e}_{e f f}$, so that

$$
B(E 2 ; J \rightarrow(J-2)) \propto\left(u^{2}-v^{2}\right)^{2} \times e_{e f f}^{2} \quad,
$$

which will again go to zero at the effective midshell.

Combined with the decrease in energy spacing as a function of increasing spin in a typical multiplet (see Sect. 4) relatively long lifetimes inevitably occur, even though the states connected by $E 2$ transitions arise from the same configuration. For the maximally-aligned spins, isomers with a wide range of lifetimes, from nanoseconds to many microseconds, can result.

An excellent example is provided by the long chain of isomers discovered in the tin isotopes $\left([51,52]\right.$ and references therein) and attributed to the $\nu h_{11 / 2}^{n}$ configuration 


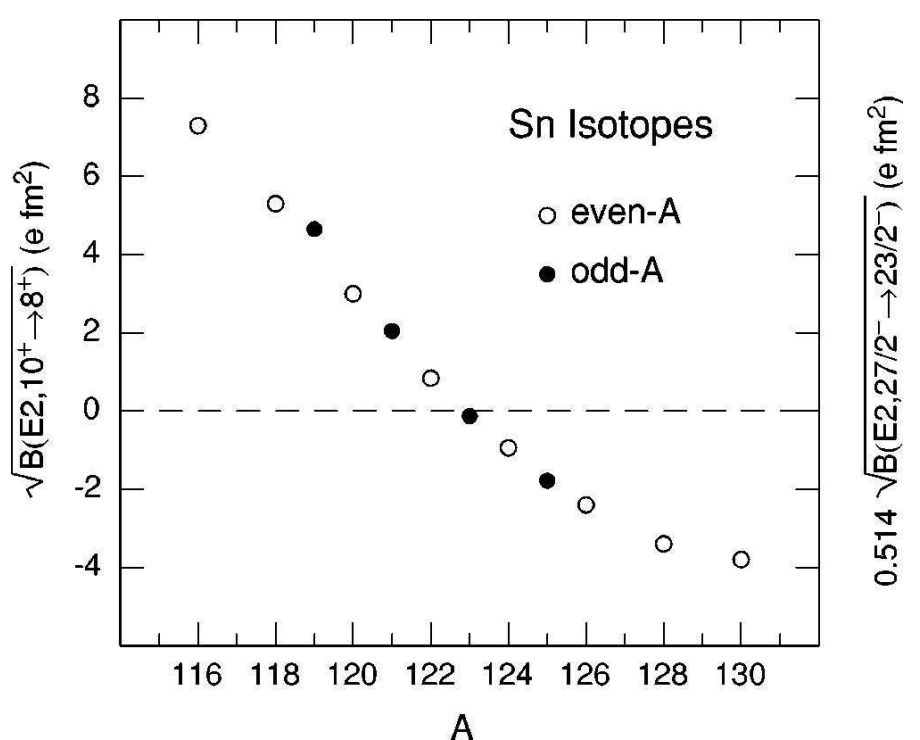

Figure 2. Compilation of $B(E 2)$ values for the $h_{11 / 2}^{2}$ and $h_{11 / 2}^{3}$ neutron chain in the Sn isotopes given by Zhang et al. [52] (see also Lozeva et al. [53]). Note the right-hand scale which is adjusted for the difference in geometrical factors for the seniority-two and seniority-three cases.

with seniority two and three. The consistency of this interpretation can be seen from the systematics of $\sqrt{B(E 2)}$ values given in figure 2: the results for the $10^{+}$and $27 / 2^{-}$ isomers in the odd and even isotopes all fall on the same locus once adjustment is made for the difference in geometric factors. (Note that the sign change at mid-shell is assumed, not measured.)

Seniority isomers can thus be used as a signature of the presence of shells or isolated sub-shells and the concomitant wave-function purity, as well as of a transition to collectivity [54]. For example, the presence of $\pi h_{11 / 2}^{n}$ isomers is part of the argument put forward for the substantive nature of the $Z=64$ sub-shell gap at $N=82$ in the neutron-deficient Dy-Er-Hf region [55, 56, 57], and was used early on to study exotic neutron-rich nuclei such as ${ }^{68} \mathrm{Ni}$, providing evidence for a shell closure at $N=40$ [58]. These isomers continue to play a significant role in the characterisation of a range of neutron-deficient and neutron-rich nuclei (see for example Refs. [59, 60, 61, 62] and Sect. 4).

Higher-spin states can usually be formed by progressively breaking paired orbits near the Fermi surface, resulting in isomers at the head of each new sequence, mimicking the procedure that leads to the lowest-seniority isomers. This is a relatively energyefficient process because of the underlying residual interactions, as will be discussed later (Sect. 4) using ${ }^{212} \mathrm{Po}$ as an example (Sect. 4.1.3). 


\subsection{K-isomers in deformed nuclei}

In nuclei which are well deformed in their ground state, states of high angular momentum can be constructed by collective rotation around an axis perpendicular to the symmetry axis. The additional breaking of pairs of particles and alignment of their individual angular momenta with the rotation, occurs when the Coriolis effects are large [63]. This is favourable for orbitals with high intrinsic spin $(j)$ and small projection of that spin on the symmetry axis $(\Omega)$. However, when $\Omega$ is large the Coriolis effects are generally small, and if both protons and neutrons have high- $\Omega$ orbitals near their Fermi surfaces, the projections can be coupled to a large total parallel to the symmetry axis,

$$
J^{\pi}=K^{\pi}=\sum_{i} \Omega_{i}^{\Pi\left(\pi_{i}\right)}
$$

at a cost in energy which is dominated by the breaking of pairs so that (to first order)

$$
E^{*} \approx \sum_{k} \sqrt{\left(\epsilon_{k}-\epsilon_{F}\right)^{2}+\Delta^{2}}
$$

where $\epsilon_{k}$ is the single-particle energy, $\epsilon_{F}$ is the Fermi energy and $\Delta$ is the pairing gap parameter, which can be obtained from odd-even mass differences. High- $K$ intrinsic states provide a large base (or bandhead) angular momentum upon which higher-spin states can be built through collective rotation. The angular-momentum coupling scheme for high- $K$ states is shown in figure 3 .

In the mass-180 region, the high- $K$ intrinsic states often compete to form the yrast line with states produced by collective rotation of lower-seniority configurations. As an example, in the odd-odd nucleus ${ }^{178} \mathrm{Ta}(Z=73, N=105)$, one can construct a three-quasiproton, three-quasineutron state at the cost of about $2\left(\Delta_{p}+\Delta_{n}\right) \sim 3 \mathrm{MeV}$. This is precisely what is seen experimentally in ${ }^{178} \mathrm{Ta}$ which has a $K^{\pi}=21^{-}$isomer at $2912 \mathrm{keV}$ and a $22^{+}$isomer at $3132 \mathrm{keV}$ [64], both of which are lower in energy than band members of the same spin based on lower two- and four-quasiparticle states. In general, and for obvious reasons, the energy favoured intrinsic states usually involve balanced excitations with similar numbers of neutrons and protons present. Few cases are known involving only four neutrons $\left(\nu^{4}\right)$, and to date, no four-proton isomers have been identified. More sophisticated approaches to the calculation of multi-quasiparticle states will be discussed in a subsequent section (3.2).

From a general nuclear-structure perspective, the high- $\Omega$ orbitals are located in the upper half of any given shell, assuming prolate deformation, thereby constraining the regions of $N$ and $Z$ where high- $K$ isomers are most likely to be observed. Furthermore, if the Fermi level is too high in a shell, the deformation weakens and axial symmetry is lost. The overall result is that $K$ isomerism is most favoured approximately two thirds of the way through a shell. For example, hafnium, with $Z=72$, is close to $70 \%$ through the $50-82$ proton shell. 

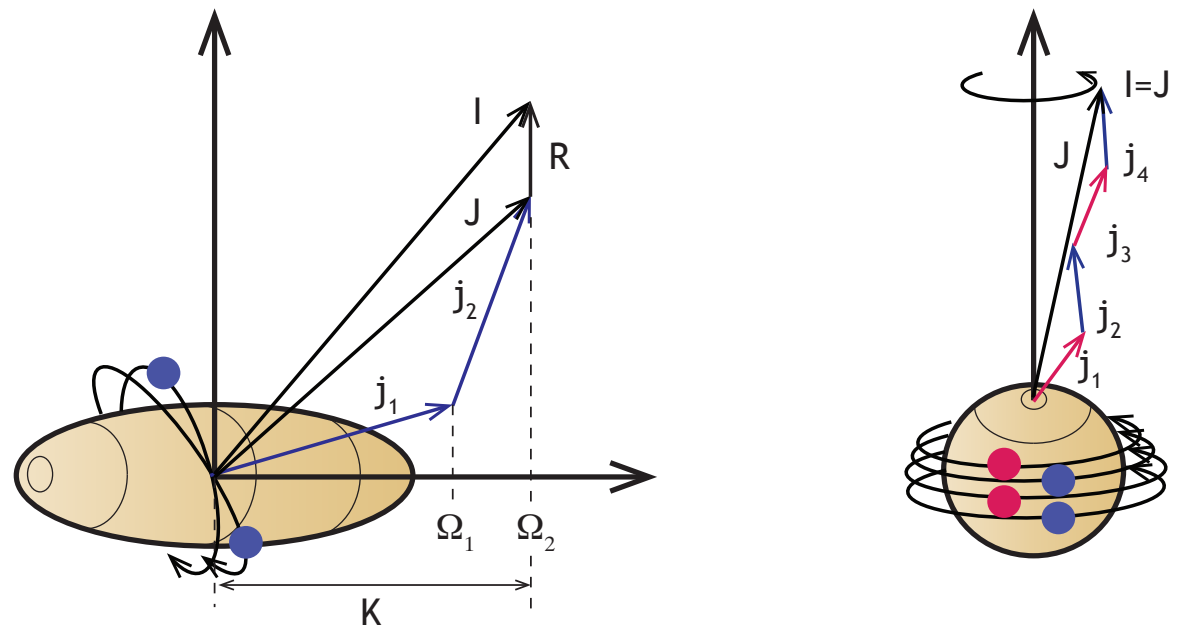

Figure 3. Schematic angular-momentum coupling schemes: on the left is an illustration of high- $K$ coupling in a deformed nucleus, where, in the absence of collective rotation $(\mathrm{R})$, the total particle angular momentum $(\mathrm{J})$ would precess around the nuclear symmetry axis. The right portion shows the contrasting scheme of many-particle alignment in a spherical nucleus.

\subsection{K-hindered decays}

The decay of high- $K$ intrinsic states is a non-collective process whose rate depends on the change in $K$ and the transition energy required to connect them to lower- $K$ states. If the difference in $K$ is larger than the multipole order $(\lambda)$, the transitions are essentially forbidden, so that isomers are formed. For configurations in which the particle-particle interactions are attractive, the corresponding energy depressions can lead to smaller energy gaps and, therefore, even slower transitions.

The degree of forbiddenness is usually classified in terms of the magnitude of the hindrance $(F)$ with respect to single-particle estimates. This hindrance is found to be strongly correlated with the degree of forbiddenness, $\nu=\Delta K-\lambda[41,65]$. A further step commonly taken involves extracting a "reduced" hindrance $f_{\nu}=F^{1 / \nu}=\left(\tau^{\gamma} / \tau^{W}\right)^{1 / \nu}$, where $\tau^{\gamma}$ is the partial $\gamma$-ray mean-life and $\tau^{W}$ is the Weisskopf single-particle estimate. While the expectation that the reduced hindrance should fall within the range $f_{\nu} \sim$ $30-300$ is regularly used as a "rule-of-thumb" for interpreting assignments and decay properties, largely based of the early analysis of Löbner [65] which showed that $f_{\nu} \sim 100$ 
for a range of $\nu$ and $\lambda$, many cases fall outside this guideline due to systematic effects and for specific reasons. It should be borne in mind that for highly forbidden transitions, small differences in $f_{\nu}$ correspond to very large differences in $F$. Furthermore, values of $F$ exceeding $10^{11-13}$ are not uncommon, implying very small $K$-admixtures.

In the broader context, with the larger body of data accumulated since the early compilation of Löbner, a more comprehensive analysis has been possible leading to improved guidelines for extracting reduced hindrances. This essentially involves factorising the total hindrance into an intrinsic component, such as the factor of $10^{3}$ or $10^{4}$ used sometimes arbitrarily for modifying $E 1$ strengths, and a $K$-forbidden component, as is discussed elsewhere [41]. The new systematics generally indicate a significantly lower value of the reduced hindrance than previously adopted [65].

\section{Deformed and transitional nuclei with $A \approx 160-190$}

In this section, the focus is on the rare-earth deformed region, extending through refractory elements up to lead. Heavier deformed nuclei are discussed in more detail in Sect. 5 .

\subsection{Multi-quasiparticle excitations and common building blocks}

Table 1. Example two-quasiparticle excitations in prolate deformed nuclei.

\begin{tabular}{cc}
\hline neutron & proton \\
\hline$Z \sim 70-74 N \sim 100-108$ & \\
\hline $6^{-}: 5 / 2^{-}[512], 7 / 2^{+}[633]$ & \\
$6^{+}: 5 / 2^{-}[512], 7 / 2^{-}[514]$ & $6^{+}: 5 / 2^{+}[402], 7 / 2^{+}[404]$ \\
$8^{-}: 9 / 2^{+}[624], 7 / 2^{-}[514]$ & $8^{-}: 9 / 2^{-}[514], 7 / 2^{+}[404]$ \\
$Z \sim 76 N \sim 110-116$ & \\
\hline $10^{-}: 9 / 2^{-}[505], 11 / 2^{+}[615]$ & \\
$10^{+}: 9 / 2^{+}[624], 11 / 2^{+}[615]$ & $10^{+}: 9 / 2^{-}[514], 11 / 2^{-}[505]$ \\
$12^{+}: 11 / 2^{+}[615], 13 / 2^{+}[606]$ & \\
\hline$Z \sim 102-108 N \sim 150-164$ & \\
\hline $8^{-}: 7 / 2^{+}[624], 9 / 2^{-}[734]$ & $8^{-}: 7 / 2^{-}[514], 9 / 2^{+}[624]$ \\
$8^{-}: 7 / 2^{+}[613], 9 / 2^{-}[734]$ & \\
$10^{-}: 9 / 2^{+}[615], 11 / 2^{-}[725]$ & $10^{-}: 9 / 2^{-}[505], 11 / 2^{+}[615]$ \\
\hline
\end{tabular}

As stated above, in regions such as $Z \sim 72, N \sim 104$, intrinsic excitations the multi-quasiparticle states - compete with collective levels based on lower-energy intrinsic states of less complexity, and the intrinsic states are often isomers.

For a number of nuclei in the Hf-Ta-W region accessible by fusion-evaporation reactions, the bandheads and associated rotational states are close enough to the yrast line for both to be populated. The structures can then be readily characterised, often because a particular spin and parity for a bandhead is only obtainable from a restricted, or even unique, combination of orbitals near the Fermi surface; the in-band decay properties can differentiate between alternative proton and neutron configurations and 
between specific orbitals; and band spacings (alignments) indicate whether component particles which suffer Coriolis effects are present. This was the rationale behind the approach and analyses of Ref. [66] aimed at isolating effects such as seniority-dependent and configuration-dependent pairing, that deduced a step-wise progression towards rigid rotation caused by blocking.

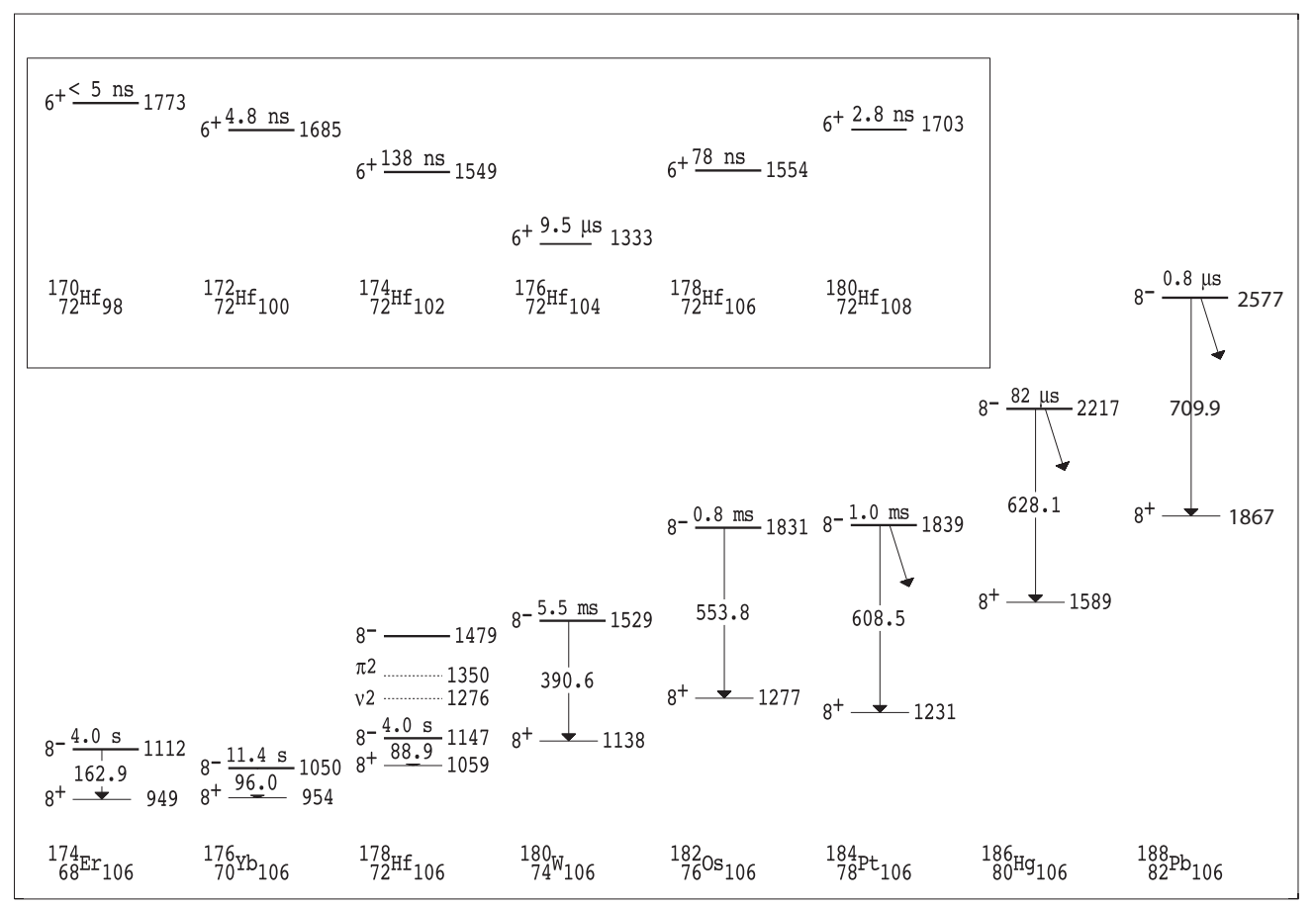

Figure 4. Half-life systematics of $K^{\pi}=6^{+}$isomers in the $Z=72$ (Hf) isotopes (inset) and the $K^{\pi}=8^{-}$isomers in the $N=106$ isotones (adapted from Ref. [41]). Level and transition energies are given in $\mathrm{keV}$. The ${ }^{180} \mathrm{Hf} K^{\pi}=6^{+}$half-life is from Tandel [68].

The two-particle configurations that recur as the main components of high- $K$ isomers in the well-deformed regions are listed in table 1 . These are combinations of the high $\Omega$ orbitals that lie near both proton and neutron Fermi surfaces at deformations of $\beta_{2} \sim 0.25$. In the hafnium region, for example, alternative pairs of $6^{+}$and $8^{-}$ configurations arise, the lower one of each pair commonly leading to a $6^{+}$and an $8^{-}$ isomer. Significant mixing occurs when the intrinsic states from the proton and neutron alternatives fall close in energy, as is the case for the $8^{-}$states in ${ }^{178} \mathrm{Hf}$. The robust nature of the $8^{-}$excitation also makes it a useful tool as a signature of prolate deformation, even in nuclei that are not necessarily deformed in their ground states. Figure 4 shows the energy systematics for the $8^{-}$isomers in the $\mathrm{N}=106$ isotones and $6^{+}$isomers in the $Z=72$ isotopes. It is remarkable that the $8^{-}$isomer occurs from the neutronrich nuclide ${ }^{174} \operatorname{Er}$ (see Ref. [67]), in all well-deformed isotones up to Os, through the transitional region $\left({ }^{184} \mathrm{Pt}\right)$, into the region of oblate-prolate shape coexistence $\left({ }^{186} \mathrm{Hg}\right)$ and finally in the very neutron-deficient isotope ${ }^{188} \mathrm{~Pb}$. In ${ }^{186} \mathrm{Hg}$ it is a signature of a prolate sub-minimum in its potential well (the ground state being soft and approximately 
oblate) while in ${ }^{188} \mathrm{~Pb}$, its observation has been taken as evidence for the existence of a prolate sub-minimum in a nucleus which exhibits triple shape coexistence [69, 70], as emphasised in the recent review of the shape coexistence phenomenon by Heyde and Wood [38]. The nature of the configuration is only disturbed in ${ }^{178} \mathrm{Hf}$, where interaction occurs with the alternative $8^{-}$state from the two-quasiproton configuration, with the result that the lower experimental state is strongly mixed, being about $64 \% \nu^{2}+36 \% \pi^{2}$ $[71,72,73]$. Despite the apparently stable character of the isomer, its lifetime varies by seven orders of magnitude from $11 \mathrm{~s}$ in ${ }^{176} \mathrm{Yb}$ to $0.8 \mu \mathrm{s}$ in ${ }^{188} \mathrm{~Pb}$, a result of the increase in energy of the main $E 1$ branch, and a gradual reduction in the reduced hindrance [74] in the less deformed nuclei.

\subsection{Multi-quasiparticle calculations}

The simple form for calculating the energies of multi-quasiparticle states (eqn. 2) needs to be modified to account for changes in the pairing correlations and the Fermi level as more and more individual orbitals are blocked. In recent years, a large number of comparisons with experiment and predictions for neutron-rich cases have been reported (see for example Ref. [37]) based on fixed deformation models in which each multiquasiparticle state is calculated separately, taking into account, self-consistently, the effect of blocking. The Fermi-level is calculated with fixed proton and neutron pairing strengths, $G_{p}$ and $G_{n}$, usually taken from the systematics of mass differences, with either a Nilsson or Woods-Saxon basis for the initial single-particle states. The models detailed in Refs. [64, 75, 76] differ mainly through the choice of either BCS pairing or the LipkinNogami method with particle-number projection. An important factor is the inclusion of spin-spin residual interactions (generalised from those that produce the well-known Gallagher-Moszkowski splittings [77, 78]) estimated where possible from empirical data. These are essentially a sum of (signed) proton-proton, proton-neutron and neutronneutron splittings. The corrections are significant and can amount to energy shifts of several hundred keV [76].

The calculations allow one to identify which configurations will lead to relatively low-lying high- $K$ states. They do not, as indicated earlier, directly predict the decay properties of isomers. A comparison of the ${ }^{177} \mathrm{Hf}$ predicted intrinsic states with the observed intrinsic states, together with associated rotational bands, is given in figure 5, on an expanded energy scale, from the work of Mullins et al. [79]. The agreement in terms of energies is typically within $\pm 150 \mathrm{keV}$ and usually better. The fact that the $37 / 2^{-}$and $39 / 2^{+}$states in ${ }^{177} \mathrm{Hf}$ lie nearly one $\mathrm{MeV}$ below the collective yrast line, represented by the $9 / 2^{+}$and $7 / 2^{-}$rotational bands with relative energies close to zero, explains their strong population. Note that ${ }^{177} \mathrm{Hf}$ is stable in its ground state and has been studied at high spin largely by incomplete-fusion reactions, so that the input angular momentum is limited. Low-lying higher- $K$ states are predicted, such as the $K^{\pi}=43 / 2^{-}$and $45 / 2^{+}$seven-quasiparticle states in ${ }^{177} \mathrm{Hf}$, that are expected to lead to isomers. 


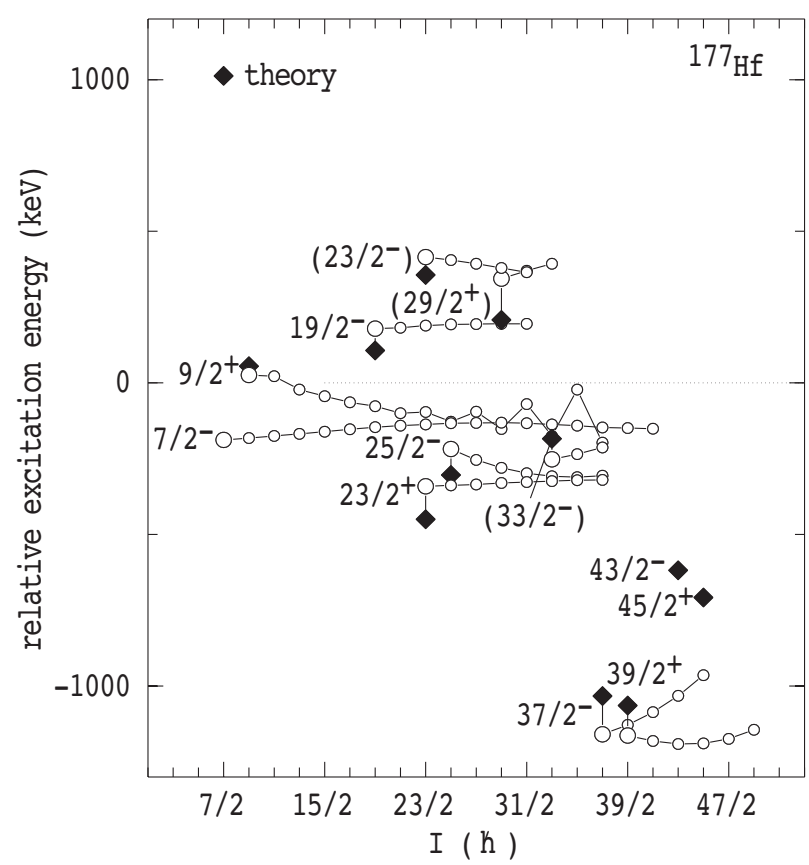

Figure 5. Energies relative to an arbitrary rigid rotor of observed states and predicted multi-quasiparticle intrinsic states in ${ }^{177} \mathrm{Hf}$.

It should be remembered that the experimental situation is ideal when the isomers are relatively short-lived ( $T_{1 / 2}$ less than a few microseconds) making it possible to correlate events above and below the isomeric states. It becomes more challenging when the state lifetimes exceed the microsecond region, resulting in separated structures that have to be treated, in terms of identification, as essentially separate nuclei.

While these models assumed fixed deformations, calculations that take into account the configuration-dependence of the deformation are likely to produce somewhat different expectations when the nuclei become softer at the deformed perimeter - in general when $N=116$ is approached. In some cases, prolate deformation tends to be stabilised by the quasiparticle excitations, such as for hafnium [80], but this is unlikely to always be the situation for transitional nuclei.

It is not surprising that the combination of the two $8^{-}$configurations leads to the very low-lying $\left(\pi^{2} \nu^{2}\right)$ four-quasiparticle $K^{\pi}=16^{+}, 31$ yr isomer in ${ }^{178} \mathrm{Hf}$. Both $K^{\pi}=8^{-}$, two-quasiparticle excitations are known in ${ }^{178} \mathrm{Hf}$ and a simple sum of their energies (1147 and $1479 \mathrm{keV}$ ) gives $2626 \mathrm{keV}$, well below the collective yrast line and not far above the experimental $K^{\pi}=16^{+}$isomer at $2447 \mathrm{keV}$. The $179 \mathrm{keV}$ difference is attributed to residual proton-neutron interactions. This demonstrates that one is dealing with excitations that are approximately independent, and thus have additive properties, justification for the validity of a quasiparticle transformation. From the Nilsson energies, one could anticipate that in the neutron-rich hafnium isotopes, as the $\nu^{2}\left[8^{-}\right]$configuration rises, a low-lying $K^{\pi}=18^{+}$intrinsic state would instead occur, formed by combining the two-proton $K^{\pi}=8^{-}$configuration and the $K^{\pi}=10^{-}$neutron 
configuration (table 1) that falls in energy. (Related predictions for the hafnium isotopes were made decades ago by Åberg [81] on the basis of a model with schematic pairing.)

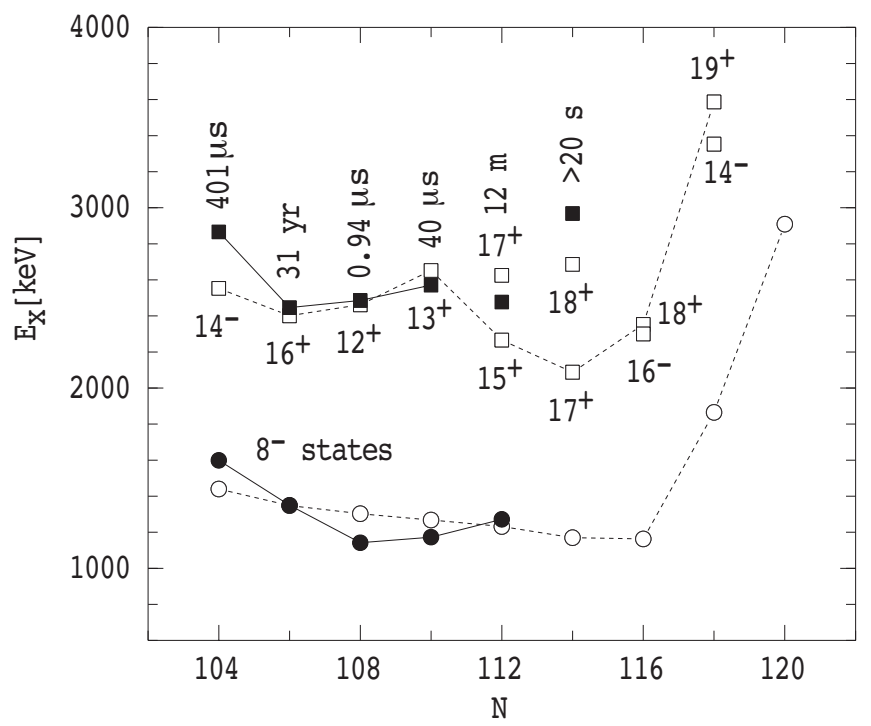

Figure 6. The lowest lying four-quasiparticle isomers in the hafnium $(Z=72)$ isotopes (filled symbols) compared to the predictions (open symbols) of the lowest states expected from configuration-constrained multi-quasiparticle calculations (adapted from Ref. [80]). Half-lives of observed four-quasiparticle isomers are given [41].

The more sophisticated multi-quasiparticle calculations such as those reported by Liu et al. [80] for the very neutron-rich hafnium isotopes (see also Sect. 3.6) allow for the configuration-dependent deformation changes expected with increasing $N$. As figure 6 shows, the two-quasiparticle $8^{-}$isomers and the lowest lying four-quasiparticle isomers, $K^{\pi}=14^{-}, 16^{+}, 12^{+}$and $13^{+}$in ${ }^{176} \mathrm{Hf},{ }^{178} \mathrm{Hf},{ }^{180} \mathrm{Hf}$ and ${ }^{182} \mathrm{Hf}$, respectively, are reasonably well reproduced, possibly with the exception of the $K^{\pi}=14^{-}$case in ${ }^{176} \mathrm{Hf}$. All of the lowest four-quasiparticle states have two neutrons added to the $\pi^{2}\left[8^{-}\right]$ core component. The $K^{\pi}=16{ }^{+},{ }^{178} \mathrm{Hf}$ case is the lowest-lying, highest-spin case (see also Refs. [17, 37]). At and beyond $N=112$, the situation is less clear, theoretically because the information on single-particle orbitals is very limited, and experimentally because current knowledge is confined to the identification of a few long-lived isomers using storage-ring techniques (see Refs. [82, 83] and Sect. 10.2.5) with little spectroscopic information. The association between predicted and experimental states in ${ }^{184} \mathrm{Hf}$ and ${ }^{186} \mathrm{Hf}\left(K^{\pi}=15^{+}\right.$and $17^{+}$possibilities in the former and $K^{\pi}=17^{+}$and $18^{+}$in the latter) is tenuous. The predicted low-lying $K^{\pi}=18^{+}$state in the $N=116$ isotope ${ }^{188} \mathrm{Hf}$, should lead to a (presumably) long-lived isomer, but this remains to be identified. It is also interesting to note that ${ }^{190} \mathrm{Hf}$ is calculated to have prolate multi-quasiparticle isomers, but the ground state is predicted to be oblate [80].

As stated before, it is not possible in any of these cases to predict a specific lifetime, 
since this is critically dependent on the decay energies and which decay paths might be open. The $K^{\pi}=10^{-}$building block involved in ${ }^{188} \mathrm{Hf}$ is a key configuration in higher- $Z$ nuclei, as are the $K^{\pi}=10^{+}$and $12^{+}$configurations, but these occur in nuclei which have competing modes of excitation at oblate, prolate and triaxial shapes, as will be discussed later (see Sect. 3.6).

\subsection{Multi-quasiparticle isomers in the mass-180 region}

There are now approximately 370 known isomers in the deformed regions, not including the one-quasiparticle cases in odd- $A$ nuclei or the two-quasiparticle cases in odd-odd nuclei [41]. Of these 370 cases, approximately $65 \%$ have well-established spins and parities and most have associated rotational bands identified, leading to firm configuration assignments [41]. As already intimated, these configurations in neighbouring nuclei - either isotopic or isotonic chains - are usually simply related, with the qualification that the additional particle might result in either an attractive or repulsive residual interaction, thus disturbing the superficial relationship between neighbours. This is more complicated in cases involving the addition of an $\Omega=1 / 2$ orbital since that can result in two intrinsic states (with $K \pm 1 / 2$ ) initially in close proximity but significantly split after the interaction.

Figure 7 illustrates multi-quasiparticle isomers in $A=175$ isobars. The isobars range from ${ }^{175} \mathrm{Re}$ (neutron deficient) to ${ }^{175} \mathrm{Tm}$ (neutron rich). The intermediate cases of ${ }^{175} \mathrm{Ta}$ and ${ }^{175} \mathrm{Lu}$ are omitted for clarity. The central nuclide is ${ }^{175} \mathrm{Hf}$, which has the isomer with the (to-date) maximum identified number of quasiparticles (nine) [84, 85]. Initially, the energy spacing varies approximately linearly with spin, reflecting that the pair-breaking energy dominates. However, it can be seen that the seven- and ninequasiaprticle states ( $K=45 / 2$ and $57 / 2$, respectively) are increasingly higher in energy than linear behaviour would suggest. This is because the high- $K$ orbitals near to the Fermi surface(s) are already singly occupied, and additional quasiparticles come from further away from the Fermi surface(s). However, this need to occupy orbitals increasingly distant from the Fermi surface(s) is not necessarily a limiting feature for the formation of very-high- $K$ isomers, because the collective energies themselves increase quadratically with spin. The collective and non-collective states are seen to be very close in energy at high spin in ${ }^{175} \mathrm{Hf}$. The high-spin limit to $K$ isomerism remains an open question.

On the neutron-deficient side of ${ }^{175} \mathrm{Hf}$, illustrated in figure 7 for ${ }^{175} \mathrm{Re}$, isomerism is weakened because the multi-quasiparticle energies tend to increase (they become further from yrast) and the deformation decreases, with both effects leading to shorter lifetimes. On the neutron-rich side, illustrated for ${ }^{175} \mathrm{Tm}$, the isomers tend to be formed at lower energies, and the dominant effect in the apparent loss of isomerism for higher $K$ values is the experimental difficulty in synthesising and studying the nuclei at high angular momentum. The corresponding situation in the hafnium isotopic chain, where particular favouring of high- $K$ isomers is calculated for neutron-rich ${ }^{188} \mathrm{Hf}$, has been 


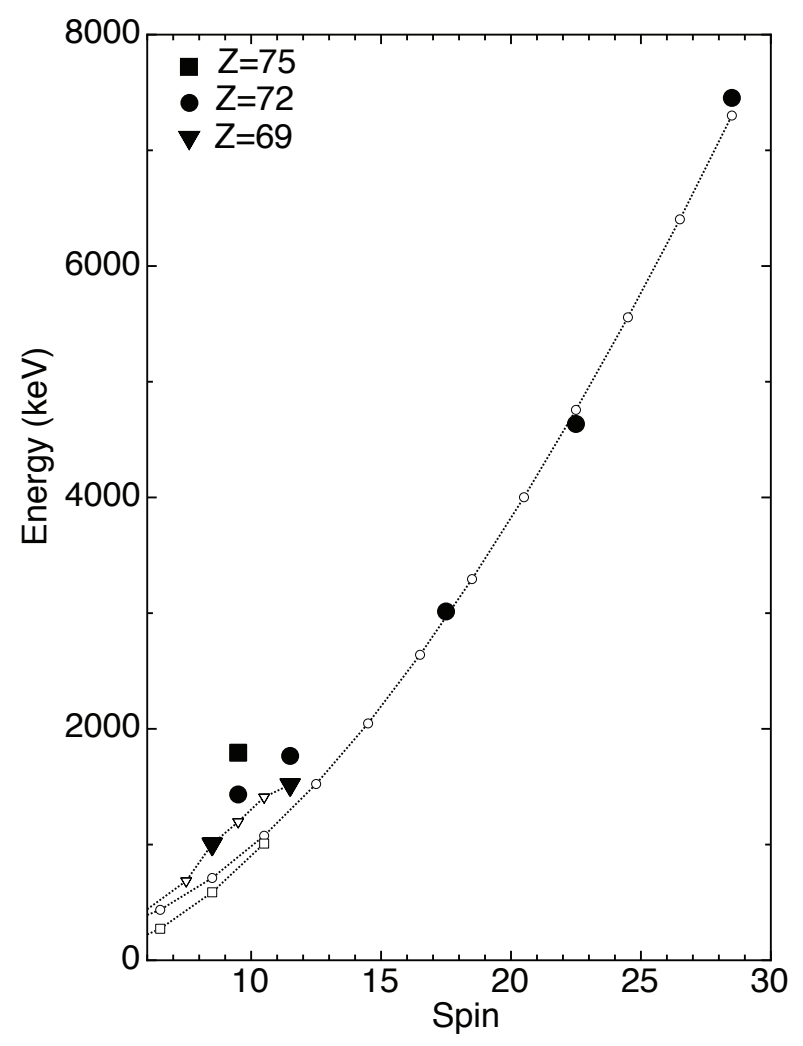

Figure 7. Multi-quasiparticle isomers with $T_{1 / 2}>1$ ns in selected $A=175$ isobars: $Z=75$ (rhenium), 72 (hafnium) and 69 (thulium). Some collective yrast states (open symbols) are also shown (mainly $Z=72$ [86]) with connecting lines. The isomer data are from Ref. [41].

discussed in the previous section. See also Sect. 10 for recent isomer discoveries.

\subsection{K-hindrances}

Numerous attempts have been made to isolate the factors which (might) govern the strength of nominally forbidden decays, although the situation is complicated since more than one factor is usually at play, and the correlations are generally masked when a broad range of data are included. This can be seen from figure 8(a) which includes the reduced hindrances for $E 2$ transitions, restricted already to cases with forbiddenness $\nu \geq 4$, plotted as a function of the product of the valence nucleon numbers, $N_{p} N_{n}$. This parameter can be taken as a global surrogate for well-localised deformation [87] in an approach promoted by Walker and collaborators [88, 89, 90, 91, 92]. There is an obvious trend of increasing $f_{\nu}$ with $N_{p} N_{n}$ at the upper boundary, but also a large number of relatively low values. A clearer situation emerges in figure 8(b) which is restricted to two- and three-quasiparticle cases in the mass-180 region. There is a distinctive correlation, with significant differences in the actual magnitudes, depending on whether the decays involve a change in the proton or neutron component of the configuration. The highest and lowest examples marked correspond to ${ }^{174} \mathrm{Yb}$ at the upper end, and 

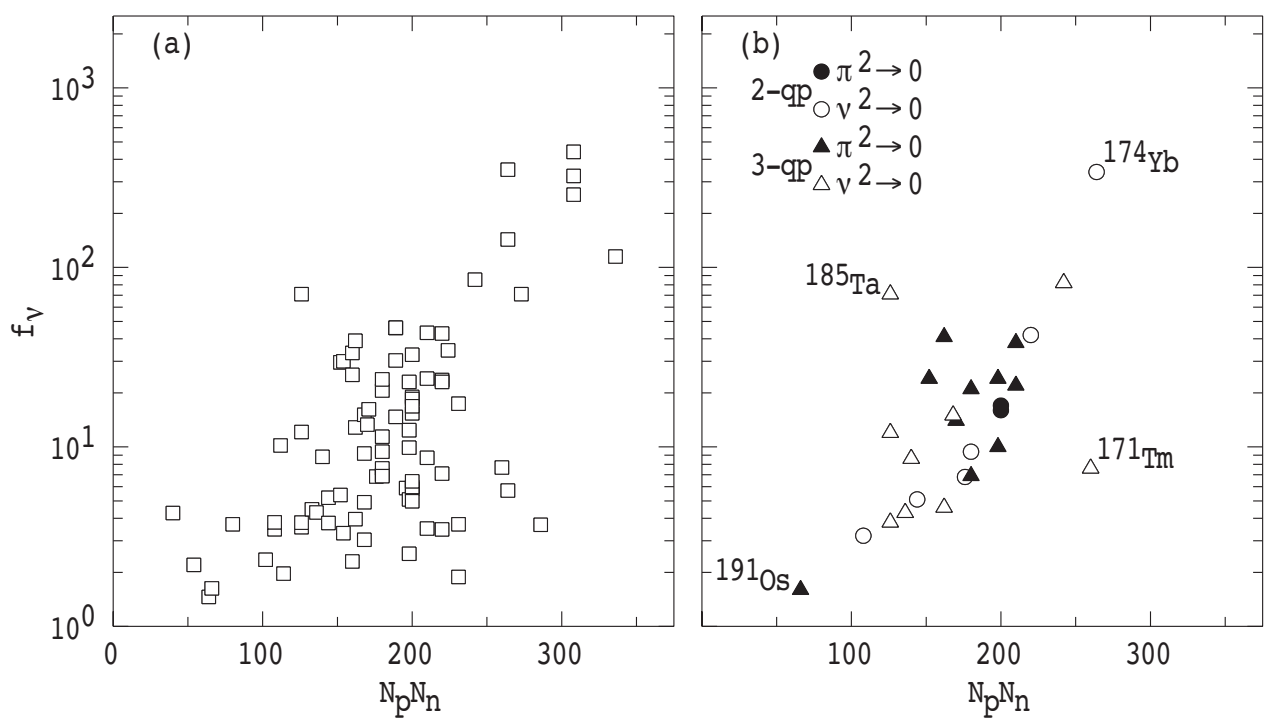

Figure 8. Reduced hindrances for a) $E 2$ decays with $\nu \geq 4$ and in the mass $170-250$ region and b) $E 2$ decays with $\nu \geq 4$ from two- and three-quasiparticle isomers in the mass 180 region. In b), proton and neutron configuration changes are distinguished by the symbols indicated (adapted from Ref. [91]).

to the transitional nucleus, ${ }^{191}$ Os at the lower end. Two "outliers" are marked: the very low value for ${ }^{171} \mathrm{Tm}$ has been explained as an example of random two-state mixing between the isomeric intrinsic state and a collective (band) state (see Ref. [93] and the following discussion); and the high value for ${ }^{185} \mathrm{Ta}$ could be due to its relatively low excitation energy [91].
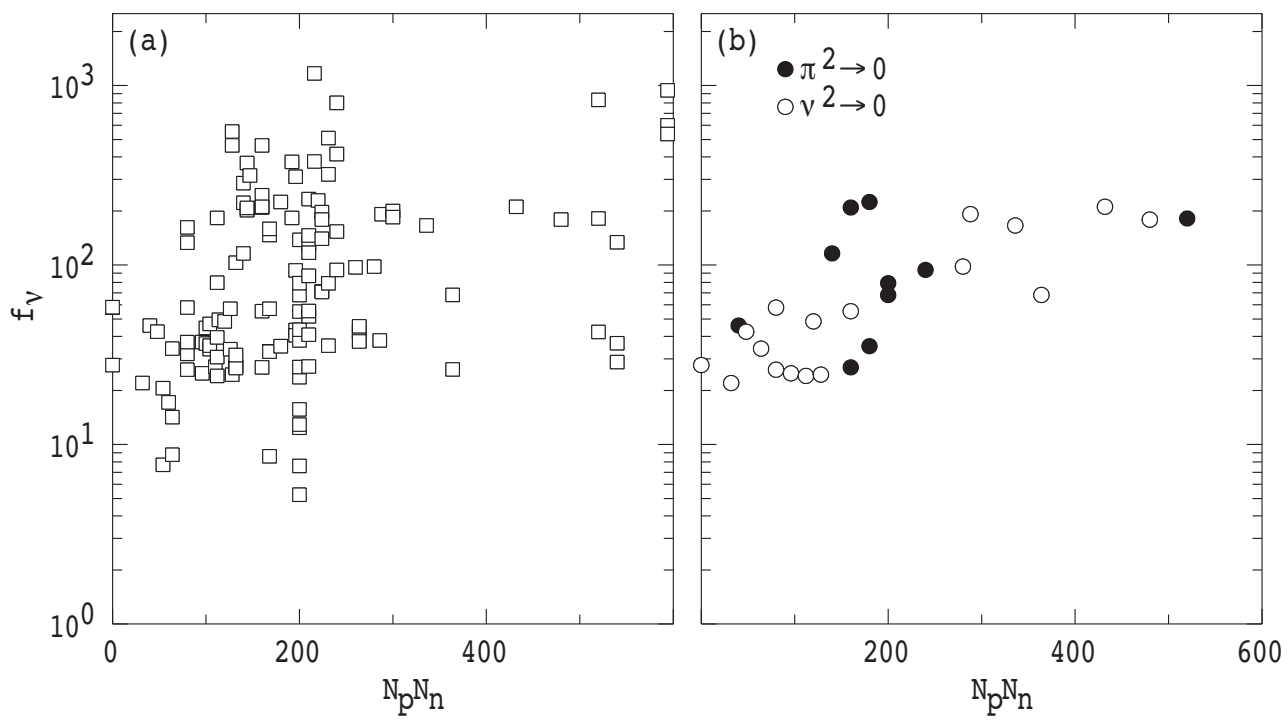

Figure 9. Reduced hindrances for a) $E 1$ decays with $\nu \geq 4$ in the mass 130-250 region and b) $E 1$ decays from $K^{\pi}=8^{-}$isomers. In b), proton and neutron configuration changes are distinguished by the symbols indicated. 
Representative $E 1$ decays are given in figure 9. Figure 9(a) is a collection of reduced hindrances for a broad mass range (including the three main deformed regions) but including only $\nu \geq 4$ transitions. Even with this constraint a large variation in $f_{\nu}$ is observed, from which it would be difficult to claim a correlation. A clearer dependence is seen for $\nu=7$ decays from the $K^{\pi}=8^{-}$states, but still with significant variation. Some of these cases differ in that proton or neutron configuration changes are involved in the decays, but again, no clear pattern emerges.

The factors which are known to control the hindrances, all related in some way to $K$-mixing, were discussed in detail in Ref. [41] and also covered in Ref. [42]. In outline, they relate to interactions which can induce mixing in either the initial or final states, or in both. For example, the cases of chance degeneracies between an isomer and a collective state of the same spin and parity result in very small admixtures that induce relatively large (collective) transition components. These cases provide a very sensitive probe of the residual matrix elements and their dependence on the $K$-difference [94]. More generally, configuration-dependent rotational mixing occurs through Coriolis effects in cases involving high- $j$ orbitals, such as the $i_{13 / 2}$ neutron. These effects are amenable to calculation and when corrections are made for the $K$-mixing, essentially normal hindrances are extracted in cases that otherwise appear anomalous [74, 94], including instances where mixing occurs in both initial and final states.

The background to the $N_{p} N_{n}$ dependence discussed earlier is that there will be a correlation between how large and well-confined the deformation is and the stiffness of the ground-state (or near ground-state) bands to which the isomers decay. This is largely a final-state effect and does not necessarily imply a correlation with the properties of the initial isomeric states. A related but more specific parameter is the ratio of dynamic and kinematic moments-of-inertia that has been associated with $K$-mixing in members of the ground-state bands (see Ref. [95] for example). This provides a strong inverse correlation with $\Delta K=8, E 1$ reduced hindrance factors [90, 95, 96].

At high level densities, less specific effects occur. High- $K$ states that lie significantly above the yrast line are likely to reside in a region where even very small interactions with the dense background of low- $K$ states will lead to an effective dilution of the $K$ quantum number. This situation is of significant interest for studies that aim to quantify the excitation-energy dependence of mixing [97]. Of more general interest is elucidation of the transition from an ordered to a chaotic quantum system (see $[98,99,100,101]$ for example) and it is useful to link $\gamma$-ray data from discrete-line studies [97] with data from quasi-continuum studies [101]. However, the connection between the two methodologies $[97,101]$ needs further investigation of the different degrees of freedom, including the choice of transition multipolarities.

The current status from discrete-line spectroscopy is represented by figure 10, with data from Kondev et al. [41] and spins ranging from $12 \hbar\left({ }^{164} \mathrm{Er}\right)$ to $57 / 2 \hbar\left({ }^{175} \mathrm{Hf}\right)$. In the figure, at least four quasiparticles are reqired for the decaying state. Note that many of the low- $f_{\nu}$ data points, that fall below the expected level-density dependence, can be understood to be affected by Coriolis $K$-mixing, related to $\left(i_{13 / 2}\right)^{2}$ neutron excitations 


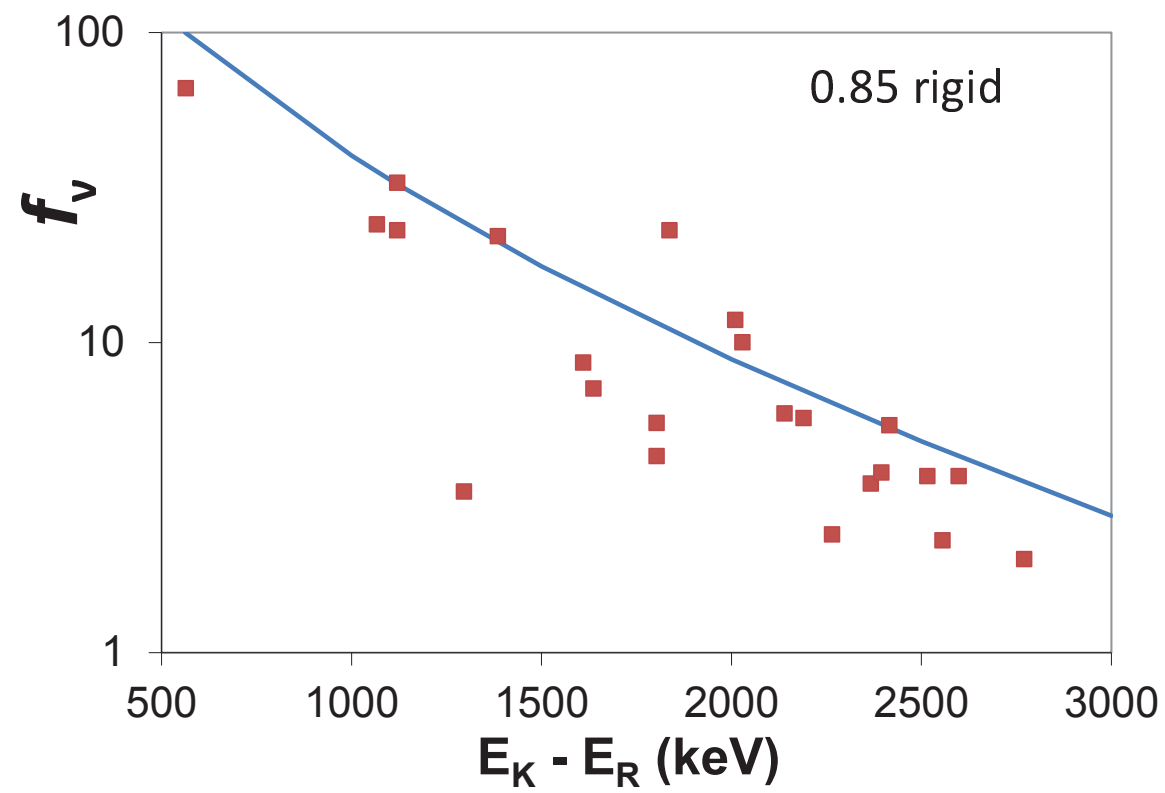

Figure 10. Reduced hindrances for $E 2$ and $E 3$ decays with $\Delta K>5$ from multiquasiparticle isomers in even-even and odd- $A$ nuclei, as a function of excitation energy relative to a rotor with $85 \%$ of the full rigid-body moment of inertia, updated from Walker [102]. At least four quasiparticles are required. For odd- $A$ nuclei, a pairing-gap energy of $0.9 \mathrm{MeV}$ has been added. The full line represents the expected level-density dependence [97]. The data are from Kondev et al. [41].

$[41,97]$. A leading issue that arises, at least for $K$-forbidden $E 2$ transitions, is how to understand the evolution of reduced-hindrance systematics, from $N_{p} N_{n}$ dependence for two-quasiparticle isomer decays (see figure 8) to excitation-energy dependence for multi-quasiparticle isomer decays (figure 10). It can be supposed that the blocking of pairing correlations plays an important role [103] but a theoretical description is lacking. Furthermore, the treatment of level density has to-date been schematic, and a microscopic approach, e.g. [104], would be beneficial.

Recent predictions for the presence of very long-lived high- $K$ isomers in superdeformed nuclei [105] (see also Ref. [106]) offer a distinct experimental challenge and may shed light on the above problems. Although the states will be very non-yrast, the lifetimes are predicted to be so long that even with significant mixing, as is bound to occur, there may be sufficient residual $K$-hindrance that the intrinsic states are still isomeric, perhaps in the nanosecond region (see Sect. 5.3). They would then constitute a highly sensitive experimental probe of the proposed mixing effects. 
Table 2. Magnetic dipole and electric quadrupole moments of multi-quasiparticle $K$ isomers, from Stone [46] unless otherwise indicated.

\begin{tabular}{cccccccc}
\hline Nuclide & $T_{1 / 2}$ & $K^{\pi}$ & $E^{*}(\mathrm{keV})$ & $\mu\left(\mu_{N}\right)$ & $Q(\mathrm{eb})$ & $\mu$ Ref. & $Q$ Ref. \\
\hline${ }^{177} \mathrm{Lu}$ & $160 \mathrm{~d}$ & $23 / 2^{-}$ & 970 & $+2.32(1)$ & $+5.71(5)$ & & \\
${ }^{173} \mathrm{Hf}$ & $20 \mathrm{~ns}$ & $23 / 2^{-}$ & 1982 & $+6.6(2)$ & & & \\
${ }^{177} \mathrm{Hf}$ & $51 \mathrm{~min}$. & $37 / 2^{-}$ & 2740 & $7.33(9)$ & & {$[110]$} & \\
${ }^{178} \mathrm{Hf}$ & $31 \mathrm{y}$ & $16^{+}$ & 2446 & $+8.16(4)$ & $+6.00(7)$ & & \\
${ }^{179} \mathrm{Hf}$ & $25 \mathrm{~d}$ & $25 / 2^{-}$ & 1106 & $7.4(3)$ & & & \\
${ }^{173} \mathrm{Ta}$ & $132 \mathrm{~ns}$ & $21 / 2^{-}$ & 1713 & $+6.51(16)$ & $6.23(18)$ & & {$[111]$} \\
${ }^{177} \mathrm{Ta}$ & $5.3 \mu \mathrm{s}$ & $21 / 2^{-}$ & 1355 & $+0.080(14)$ & & & \\
${ }^{176} \mathrm{~W}$ & $41 \mathrm{~ns}$ & $14^{+}$ & 3747 & $+6.7(2)$ & $6.0(8)$ & & \\
${ }^{179} \mathrm{~W}$ & $750 \mathrm{~ns}$ & $35 / 2^{-}$ & 3348 & & $+3.9(10)$ & {$[108]$} & \\
${ }^{182} \mathrm{Re}$ & $82 \mathrm{~ns}$ & $16^{-}$ & 2256 & $+3.82(13)$ & & & \\
${ }^{182} \mathrm{Os}$ & $146 \mathrm{~ns}$ & $25^{+}$ & 7050 & $+10.6(2)$ & $4.2(2)$ & & \\
\hline
\end{tabular}

\subsection{Static electromagnetic moments of multi-quasiparticle isomers}

Key information about the structure of high- $K$ isomers comes from magnetic dipole moments in combination with electric quadrupole moments. For example, rotational bands associated with high- $K$ states yield experimental $\gamma$-ray mixing ratios and branching ratios, from which (based on the rotational model) the quantity $\left(g_{K}-g_{R}\right) / \mathrm{Q}_{0}$ may be deduced (see for example Refs. [75, 107]) where $g_{K}$ is the intrinsic gyromagnetic ratio ( $g$-factor), $g_{R}$ is the rotational $g$-factor and $\mathrm{Q}_{0}$ is the intrinsic quadrupole moment. Often, assumptions about the values of $g_{R}$ and $\mathrm{Q}_{0}$ are made, enabling "experimental" $g_{K}$ values to be determined, and compared with Nilsson model predictions for specific (multi)-quasiparticle configurations. Even limited precision may be sufficient to distinguish between competing configurations, leading to this approach being widely used and of essential value.

Additional insights come from the determination of the static electromagnetic moments [18] of the isomers themselves. Data for multi-quasiparticle states are summarised in table 2. In terms of configurations these results have not yielded any particular surprises. Rather, they have generally confirmed the proposed configurations or distinguished between different alternatives. The measured state $g$-factors, however, are dependent on $g_{K}$ and $g_{R}$, through the relation

$$
g=g_{R}+\frac{K^{2}}{I(I+1)}\left(g_{K}-g_{R}\right) .
$$

If the properties of the associated rotational band are also known, and if the quadrupole moment $\mathrm{Q}_{0}$ is known or can be estimated, an independent value of $g_{R}$ can be obtained. Since the magnitude of $g_{R}$ will depend on the partition of collective motion between the proton and neutron fluids (see for example Ref. [66]), which is itself dependent on the magnitude of the pairing, the extraction of $g_{R}$ as a function of the number of quasiparticles may provide a useful test of the effect of blocking and the possible quenching of pairing in multi-quasiparticle states. Measurements in ${ }^{179} \mathrm{~W}$, which allow 
a comparison between related three- and five-quasiparticle states in the same nucleus [108], suggest that static pairing is quenched with as few as three neutron orbitals blocked. A similar analysis applied to a range of nuclei has been reported recently by Stone et al. [109], resulting in the suggestion of a surprisingly strong systematic behaviour of $g_{R}$.

By implantation into a (non-cubic) osmium crystal, the electric quadrupole moment was also obtained [112] for the ${ }^{182} \mathrm{Os} 25^{+}$isomer. This is an especially interesting case, because the ${ }^{182}$ Os isomer has an anomalously fast decay to the yrast band [113]. The low quadrupole moment measured is consistent with the theoretically predicted $\gamma$-soft shape [114]. In such a case, fluctuations will break axial symmetry and may thus enhance the otherwise $K$-forbidden decay rate, although a quantitative relationship between $\gamma$ asymmetry and $K$-mixing in such cases is not yet available.

The technique of LEvel Mixing Spectroscopy (LEMS), whereby combined magnetic and electric fields enable the ratio of the magnetic and electric moments to be obtained, has been applied [115] to the $35 / 2^{-}$isomer in ${ }^{179} \mathrm{~W}$. Taken together with the measurement of time-dependent perturbed angular distributions (TDPAD) which yield the magnetic dipole moment, as discussed above [108], the electric quadrupole moment can also be deduced [115]. The result reported for the quadrupole moment is substantially smaller than theoretical evaluations, which do not predict any significant difference in shape between lower-spin states and the five-quasiparticle isomer. This difference remains to be understood theoretically, if the experimental result can be substantiated.

Quadrupole moment measurements are not readily accessible, but for longer halflives, of milliseconds or more, laser hyperfine spectroscopy offers a powerful method that can yield both electromagnetic moments and mean-square charge radii. To date, the only multi-quasiparticle (seniority greater than three) isomer properties determined by laser spectroscopy are $[116,117]$ those of the $16^{+}, 31 \mathrm{yr}$ isomer in ${ }^{178} \mathrm{Hf}$. This is the highest-seniority isomer for which both the quadrupole moment and the charge radius have been determined. The charge radius is also known for the ground state. The observation that the isomer has a slightly smaller (mean square) charge radius than the ground state is suggested to be a pairing effect from the blocking of four quasiparticles, which leads to a lower surface diffuseness, an effect subsequently explored with a number of theoretical approaches $[118,119]$. Further measurements of isomer charge radii may soon be achieved, if recent experimental advances $[120,121]$ can be exploited.

\section{6. $A \sim 190$; transitional nuclei}

As the neutron approaches $N=116$ and the proton number increases past the sub-shell gap at $Z=76$, the magnitude of the deformation tends to decrease and vibrational structures fall to lower energies. The $N=116$ nuclide ${ }^{192} \mathrm{Os}$, for example, has the lowest $\gamma$-vibrational state in the region at $E\left(2_{\gamma}^{+}\right)=489 \mathrm{keV}$, while the nominally-prolate ground-state-band $2^{+}$state has risen to $206 \mathrm{keV}$. The region near $N=116$ and $Z=78$ 
is well-known for a prolate-to-oblate shape transition [122] and potential-energy-surface calculations with mean-field models (see for example Refs. [123, 124]) have catalogued expected deformation changes across the region. The theoretical results of Wheldon et al. [125] predict a prolate but very soft potential minimum for the ground state in ${ }^{190} \mathrm{Os}$ (see figure 11) with a transition that develops through ${ }^{192}$ Os and ${ }^{194}$ Os, in which dual mimima are seen, connected through a valley in the $\gamma$ direction, progressing on to a well-defined oblate minimum in the neutron-rich isotope ${ }^{196} \mathrm{Os}$.

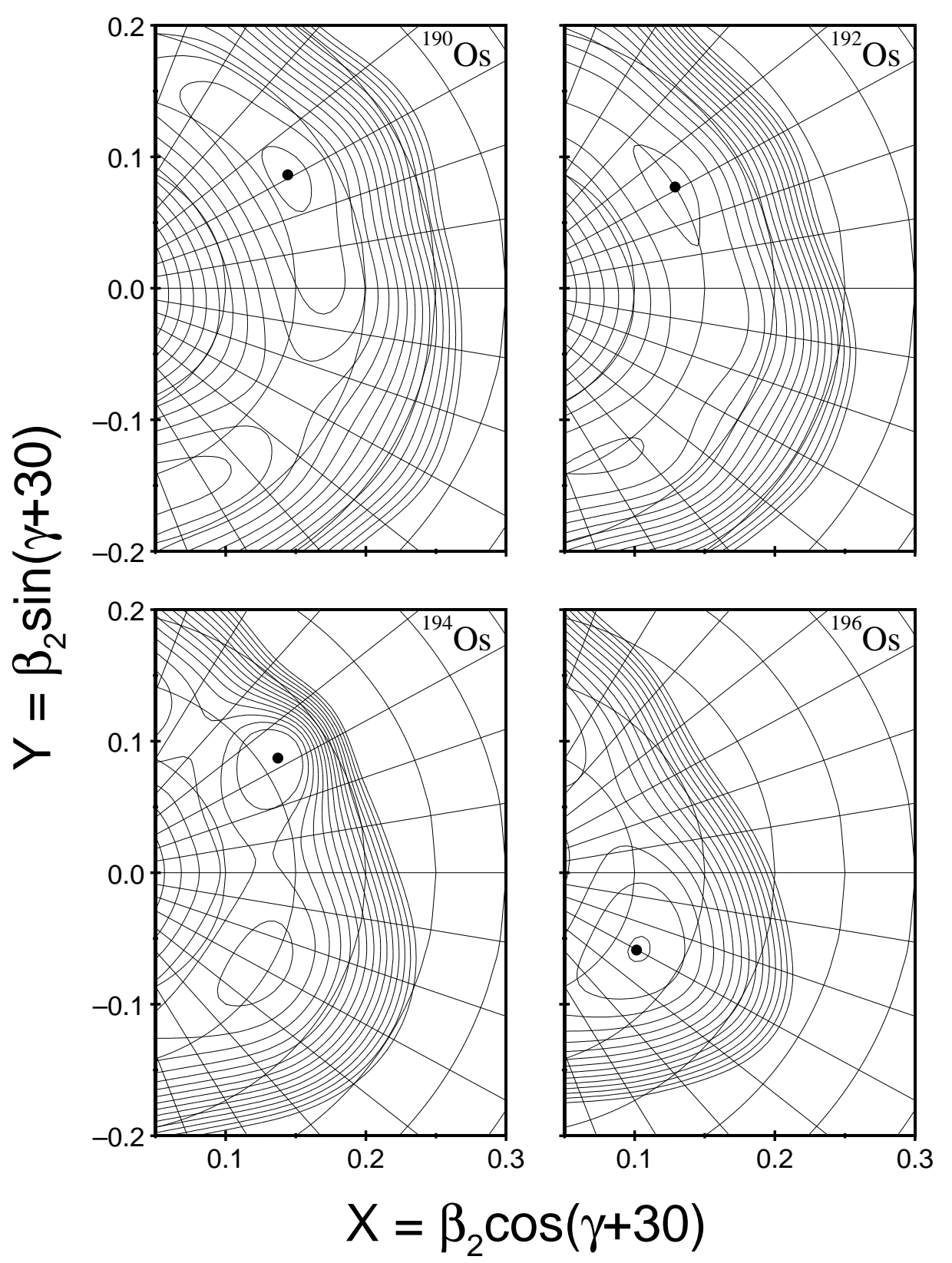

Figure 11. Potential energy surfaces for even-even osmium isotopes, ${ }^{190-196}$ Os, illustrating the transition between prolate and oblate shapes, from Ref. [125]. 
It should be noted that most broad-range calculations relate to the ground states and thus might not be relevant to multi-quasiparticle isomers, since the preferred deformation is bound to be sensitive to the population of specific orbitals, and to the dynamics introduced by rotation. The multi-quasiparticle structures that could lead to isomers are very likely to have shapes different to those occurring at low seniority. These configuration-dependent effects are not simple to incorporate in a general theory and they imply a need for analyses on a case-by-case basis.

In the more tractable cases of axial symmetry, the governing factors that define the main structures expected at high spin depend largely on the relative positions of the Fermi surfaces amongst the Nilsson orbitals. The proton Fermi surface is near the top of the unique-parity $h_{11 / 2}$ shell, thus prolate high-spin (high- $K$ ) structures are likely to involve the high- $\Omega, 11 / 2^{-}[505]$ orbital. In contrast, the proximity to the low- $\Omega$, $1 / 2^{-}[550]$ orbital at oblate deformation favours rotation-aligned structures, a maximum of $10 \hbar$ of angular momentum being available from the complete alignment of a pair of $h_{11 / 2}$ protons. Proton alignment can also occur at prolate deformation by population of the $h_{9 / 2}$ low- $\Omega$ orbitals that intrude from above the $Z=82$ shell. In the case of the neutrons, the Fermi level relative to the $i_{13 / 2}$ neutron shell is in a position analogous to that of the protons in the $h_{11 / 2}$ shell, resulting in the possibility of high- $K$ prolate structures, with a maximum of $K=12 \hbar$ obtained by summing the $11 / 2^{+}[615]$ and $13 / 2^{+}[606]$ orbital projections, or of oblate rotation-aligned structures with the same angular momentum (12 $\hbar$ ) being available from the alignment of a pair of $i_{13 / 2}$ neutrons. The net result will be a competition between different modes and configurations at prolate and oblate deformation (see Sect. 3.6.1).

Fluctuations or shifts in the triaxial direction mean that the projection is not well-defined implying that $K$ will not be a "good" quantum number. This is often stated, but in contrast to rotational effects in well-deformed axially-symmetric nuclei, the elucidation of $K$-distributions when $\gamma$ fluctuations are present is not well developed quantitatively. One qualitative expectation is that $K$-hindrances will be diluted, but this expectation is not necessarily well correlated with the existence of isomers, or with how long their lifetimes might be.

3.6.1. Isomers and transition strengths in transitional nuclei Experimentally, a distinct progression is seen in, for example, the $\mathrm{W}$ isotopes. Clear rotational structures are observed in ${ }^{188} \mathrm{~W}(N=114)$ including several two-quasiparticle high- $K$ isomers [126] while the $N=116$ isotope, ${ }^{190} \mathrm{~W}$ has an anomalous $E_{4^{+}} / E_{2+}$ ratio and a longlived isomer [127] attributed to the $K^{\pi}=10^{-}$two-neutron building block. The long half-life of $166 \mu \mathrm{s}$ is now explained as an allowed $M 2$ transition to a $K^{\pi}=8^{+}$state rather than a $K$-hindered decay [126]. Unlike the ${ }^{188} \mathrm{~W}$ case, because of the long halflife the associated rotational band for the $10^{-}$isomer, which could provide a test of the proposed configuration, has not been identified, and only a fragment of the $8^{+}$band has been found [126].

In the Os isotopes, the $10^{-}$, two-neutron (prolate) state produces long-lived 
isomers in both ${ }^{190} \mathrm{Os}$ and ${ }^{192} \mathrm{Os}$, while in ${ }^{190} \mathrm{Os}$, a $K^{\pi}=7^{-}$isomer from the $\nu 3 / 2^{-}[512], 11 / 2^{+}[615]$ prolate Nilsson configuration has been assigned [128, 129]. Notably, in ${ }^{192}$ Os $J^{\pi}=12^{+}$and $20^{+}$isomers have been identified and characterised as arising from rotation alignment of the $i_{13 / 2}^{2}$ neutrons at oblate deformation: the nearmaximal alignment gains result in $E 2$ transitions that are collectively enhanced but are low in energy [129]. Several high- $K$ prolate isomers are predicted but not, as yet, observed (see Sect. 3.6.2).

Other isomers occur in the transitional region that arise from effects such as spintrapping or rotation alignment. The former is proposed to be the case for the isomers discovered in the stable iridium isotopes, ${ }^{191} \mathrm{Ir}$ and ${ }^{193} \mathrm{Ir}$ [130]. Each has a long-lived $J^{\pi}$ $=31 / 2^{+}$isomer $\left(T_{1 / 2}=5.7 \mathrm{~s}\right.$ and $125 \mu$ s respectively $)$ that eventually decays into the triaxial $11 / 2^{-}$[505] one-quasiparticle band. On the basis of $K$-constrained potentialenergy-surface calculations with a non-axial deformed Woods-Saxon potential similar to those of Refs. [80, 114], Ref. [130] concluded that the most likely three-quasiparticle configurations giving rise to these isomers, were triaxial, with $\gamma \sim 27^{\circ}$ and $\beta_{2}=0.157$ in ${ }^{191} \mathrm{Ir}$ and $\gamma \sim 14^{\circ}, \beta_{2}=0.135$ in ${ }^{193} \mathrm{Ir}$, both arising from the $h_{11 / 2}$ proton coupled to the $10^{-}$, two-neutron core (again one of the building blocks of Sect. 3.1). These states are forced to decay by high-multipole transitions such as E3 and $M 3$. No other isomeric states have been identified in these cases, but the experimental situation is complicated by the long lifetimes that make it difficult to establish feeding transitions or higher-lying isomers.

Considering $K$-forbidden decays, there is increasing experimental evidence that the $K$-hindrances are reduced in nuclei that are susceptible to $\gamma$ vibrations [131]. For example, low $f_{\nu}$ values that have been observed for decays from states that superficially resemble $K$-isomers in the heavier tungsten and osmium isotopes, such as ${ }^{188} \mathrm{~W},{ }^{190} \mathrm{~W}$ and ${ }^{190,191,192}$ Os, are discussed by Kondev et al. [41]. Note that there are other cases discussed in the literature where the perceived absence of isomers is taken as evidence for triaxial effects, but this is less easy to justify or quantify.

3.6.2. Predictions of low-lying high-K intrinsic states The observation in the osmium isotopes of states associated with oblate deformation and the predicted dynamical effects due to rotation alignment, together with prolate high- $K$ isomers, is generally in line with expectations. However, a number of other high- $K$ multi-quasiparticle states at prolate deformation, specifically associated with the $K^{\pi}=12^{+}$configuration from the $i_{13 / 2}^{2}$ neutrons, are predicted to be low in energy [129]. It was also shown from configurationconstrained calculations that in the ${ }^{191} \mathrm{Ir}$ and ${ }^{193} \mathrm{Ir}$ cases, related high-spin intrinsic states would fall into the region of the observed isomers, and perhaps lie even lower in energy than the $J^{\pi}=31 / 2^{+}$states [130]. These are $K^{\pi}=33 / 2^{-}$and $35 / 2^{-}$levels formed from the $9 / 2^{-}[514]$ and $11 / 2^{-}[505], h_{11 / 2}$ proton orbitals also coupled to the prolate $12^{+}, i_{13 / 2}^{2}$ neutron configuration.

The predicted rapid fall in energy with neutron number is illustrated for the osmium cases in figure 12. In fact, the potential minima in the osmium cases are not purely 


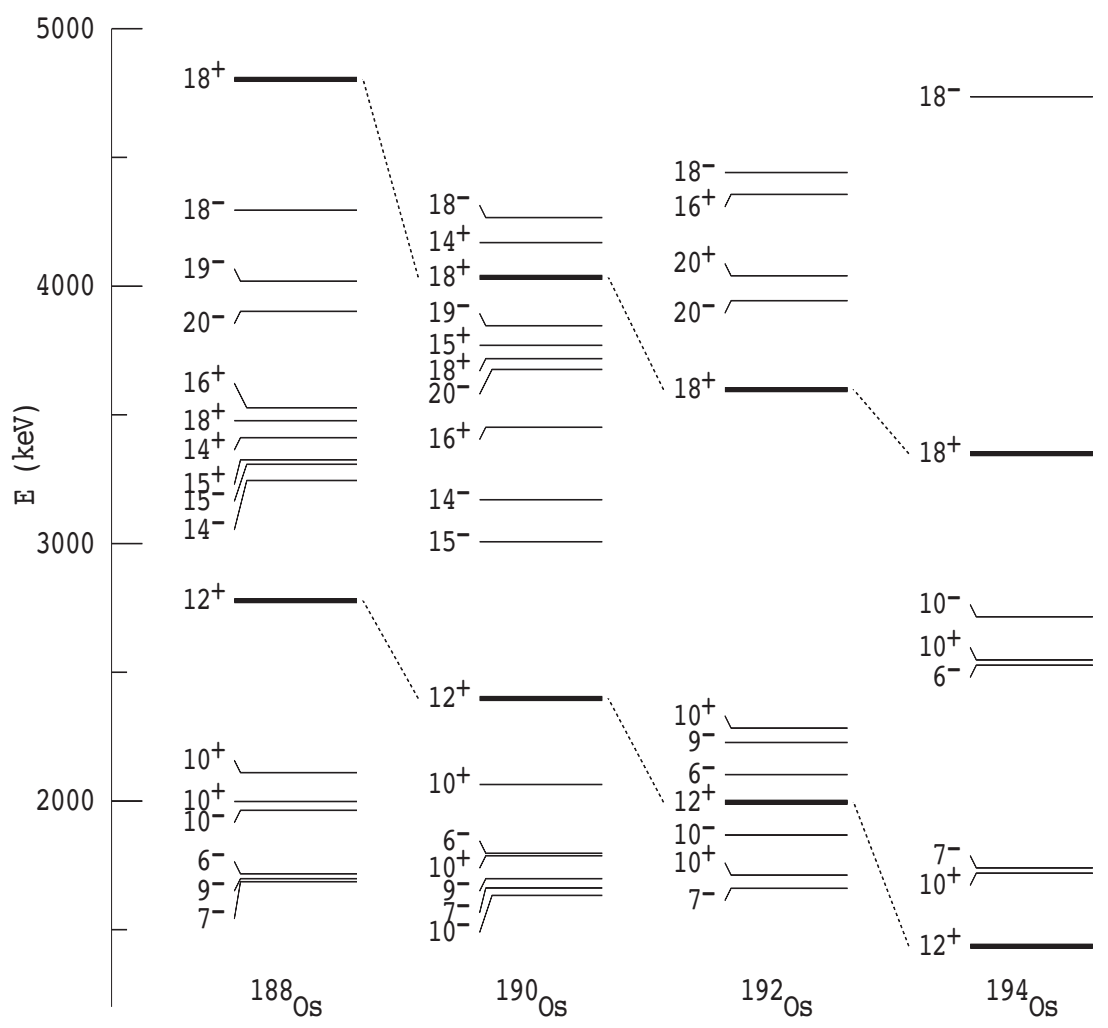

Figure 12. Predicted multi-quasiparticle states calculated using configurationconstrained potential-energy surfaces with a Woods-Saxon potential, from Ref. [129]. Each configuration has been independently minimised with respect to $\beta_{2}, \gamma$ and $\beta_{4}$ deformations.

prolate but have varying amounts of triaxiality. Aside from that factor, as can be seen from figure 12 , the $12^{+}$states are predicted to fall almost linearly, to the extent that in ${ }^{192}$ Os, and even more so in ${ }^{194} \mathrm{Os}$, they would lie below any states, intrinsic or collective, to which they could reasonably decay. Similarly, the related $18^{+}$state produced by coupling the $3 / 2^{-}[512]$ and $9 / 2^{-}[505]$ neutrons to the $12^{+}, i_{13 / 2}^{2}$ configuration is predicted to fall in a parallel fashion.

If these and the equivalent levels in the iridium isotopes indeed exist, they should be strongly populated in deep-inelastic or fragmentation reactions since they would lie well below the collective yrast line. They would only decay via $\beta^{-}$emission and thus be long lived. Presumably, the isomers would be evidenced by their long-time components in the population of excited states in the neighbours, although, experimentally, such decays might not have a simple signature, depending on disposition of levels in the daughters. This problematic situation leads naturally into a consideration of the prospects for the identification of such isomers using storage-ring techniques, techniques which do not rely on the identification of specific decays (see Sect. 10.2.5). 


\section{Spherical regions}

The main factors that govern the high-spin nuclear structure and the formation of isomers around the closed shells are the availability of high- $j$ proton and neutron orbitals and their coupling to vibrational collective modes. Major advances have been made with large-scale shell models that are now able to carry out unrestricted calculations in the full $f p$ shell, see for example Ref. [132]. In the heavier nuclei, particle-particle and particlehole interactions have been determined over a broad proton and neutron orbital space (see for example Ref. [133] and references therein). Use of these interactions has resulted in good reproduction of level schemes for nuclei near the $Z=50$ and $Z=82$ closed shells, particularly those involving only a few valence particles. This success encompasses the treatment of high-spin states when one type of valence particle is involved, such as the case of proton chains with a closed $N=126$ shell [132] as well as situations when valence protons and neutrons of the same type (all holes or all particles) are active. The most extensive application (in a formal sense) is the calculation of states in ${ }^{208} \mathrm{~Pb}$ itself by Brown [134], with excitations up to two-particle-two-hole within a 24-orbit model space. Good reproduction was obtained of both the low-lying energy spectrum and the distribution of quadrupole and octupole strength, using, of necessity, effective charges. However, extension to cases where both valence particles and also (particle-hole) core excitations occur, as is common in the formation of isomers with spins above $20 \hbar$ in the trans-lead region, is yet to be achieved $[135,136]$, hence the continuing reliance on the semi-empirical shell model (Sect. 4.1.1).

A key factor is the coupling to the $3^{-}$octupole vibration near $Z=64$ (Sect. 4.2) but more importantly, near $Z=82$ (with the $3^{-}$state at $2.615 \mathrm{MeV}$ in ${ }^{208} \mathrm{~Pb}$ ) which can be amplified for specific multiparticle configurations (Sect. 4.1.2). Finally, whether deformation plays a role is a continuing question. This is a natural expectation given that increasing numbers of particles congregate near the nuclear equator as high angular momentum states are formed by the mutual alignment of the spins of individual nucleons. This idea is captured in various versions of the deformed independent particle model (DIPM) [137, 138, 139, 140, 141] as will be discussed with reference to both the radon region and the gadolinium region (Sects. 4.1 .9 and 4.2, respectively).

As more protons and neutrons are added to the ${ }^{208} \mathrm{~Pb}$ core, a transition occurs to the regime of octupole deformation manifested in the Ra-Th nuclei, where collective modes dominate and multi-particle isomers are rare [142]. Even before reaching this region, subtle changes occur in the $E 2$ collectivity of structures that otherwise have properties that resemble shell-model isomers [143], and in the strength of characteristic decays such $j_{15 / 2} \rightarrow g_{9 / 2} E 3$ transitions in the $N=127$ isotones [144] which increase, implying a fall in energy and increase in collectivity of the core $3^{-}$vibration. This proposition is partly supported by the low $3^{-}$energy of $1687 \mathrm{keV}$ observed in ${ }^{216} \mathrm{Th}$ [145]. 
4.1. Nuclei near ${ }^{208} \mathrm{~Pb}$

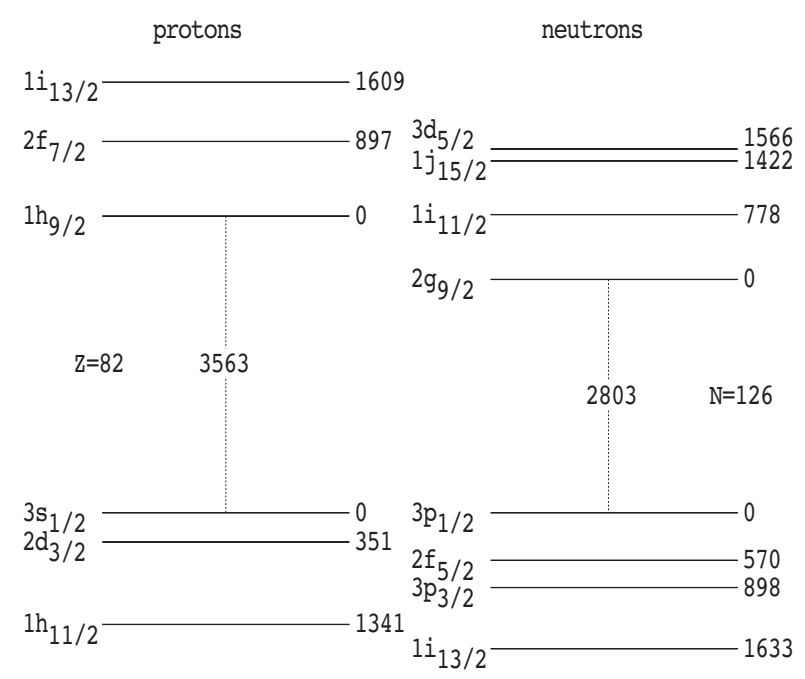

Figure 13. Relative energies of single-particle orbitals near the ${ }^{208} \mathrm{~Pb}$ doubly closed shell.

The relative energies of single-particle excitations around the doubly-closed ${ }^{208} \mathrm{~Pb}$ core are shown schematically in figure 13. As is the case with deformed nuclei, particular component configurations regularly occur in the formation of high-spin states and highspin isomers. Some of these are listed in table 3, including the important neutron-core excitations that play a major role at spins above $\sim 20 \hbar$.

Table 3. Common two-particle excitations in spherical nuclei near $Z \sim 82$ and $N \simeq 126$.

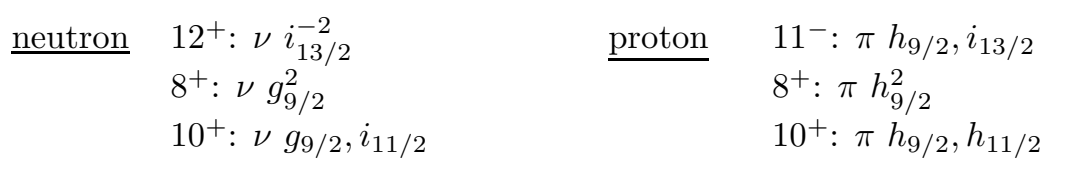

$$
\begin{aligned}
& 5^{-}: \nu p_{1 / 2}^{-1}, \frac{\text { neutron-core excitations }}{g_{9 / 2}} 7^{-}: \nu f_{5 / 2}^{-1}, g_{9 / 2} \\
& 6^{-}: \nu p_{1 / 2}^{-1}, i_{11 / 2} \quad 8^{-}: \nu f_{5 / 2}^{-1}, i_{11 / 2} \\
& 8^{+}: \nu p_{1 / 2}^{-1}, j_{15 / 2} \quad 10^{+}: \nu f_{5 / 2}^{-1}, j_{15 / 2}
\end{aligned}
$$

4.1.1. Residual interactions and the empirical shell model (ESM) As was discussed in Sect. 2.2, the basic case of two identical particles in a single- $j$ shell interacting through a short-range attractive interaction, such as a $\delta$ force or a quadrupole-quadrupole force [146], leads naturally to isomers. The $0^{+}$state in which the particle angular momenta are anti-aligned (or more generally, paired) is depressed in energy, and a gradual bunching of 
levels up to the maximum spin of $2 j-1$ occurs. As well as the small energy gap between the $j$ and $j_{\max }-2$ states, cancellation effects peak at mid-shell and reduce further the $E 2$ transition rate connecting them. These features persist for more complicated interactions and for larger numbers of particles.

The ESM approach to calculating spectra that originated with Blomqvist [147] relies on the fact that, for simple configurations in nuclei near closed shells, the effective interaction can be extracted from experimental multiplet energies measured relative to the one-nucleon spectra in adjacent nuclei. These interactions are generally well understood from the study of two-particle multiplets [148, 149, 150, 151, 152, 153, 154]. The dependence of the interaction on the alignment of the angular momenta can be viewed semi-classically as a preference for co-planar orbits intended to maximise the overlap. This overlap of orbitals (which are more confined spatially for higher $j$ ) can be parametrised as a function of the classical angle of relative orientation $\theta_{12}$ between the individual spins of the two particles:

$$
\cos \theta_{12}=\frac{J(J+1)-j_{1}\left(j_{1}+1\right)-j_{2}\left(j_{2}+1\right)}{2 \sqrt{j_{1} j_{2}\left(j_{1}+1\right)\left(j_{2}+1\right)}}
$$

Typical residual interactions are shown in figure 14 . The $j^{2}$ interaction for like particles (illustrated for $g_{9 / 2}$ neutrons) results in a bunching of levels as they approach the maximum spin $J_{\max }=2 j-1=8$.

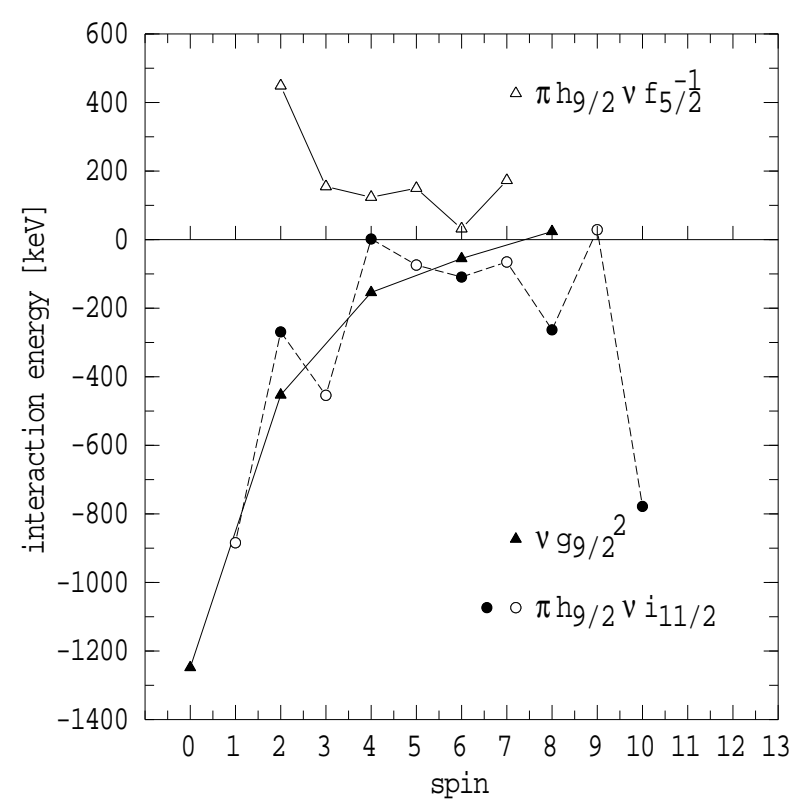

Figure 14. Examples of residual interactions near $N \sim 82$ and $Z \sim 126$.

The proton-neutron interaction for the $\pi h_{9 / 2} \nu i_{11 / 2}$ combination is strongly attractive, giving an energy gain of nearly $800 \mathrm{keV}$ when the proton and neutron angular momenta are fully aligned. In contrast the proton-neutron-hole interaction is generally repulsive if the particle spins are parallel or anti-parallel. 
To reiterate, the energy gain from the residual interaction is maximised between pairs of particles of the same character: that is, if they are both protons or neutrons, or both particles or holes. The proton-neutron interaction is also attractive under the same circumstances. In contrast, the particle-hole interaction is repulsive. Mutual alignment of the angular momentum vectors of a high-spin particle and a high-spin hole is thus not favoured. The treatment of many-particle cases requires more complicated angular momentum recoupling (see Ref. [155] for examples) but the main features are retained.

More subtle dynamical effects lead to the observation in some mercury and lead nuclei of so-called "shears bands" $[156,157,158]$. These originate essentially from proton particles coupled to maximum spin, and neutron holes coupled to maximum spin, but with those two components arranged at the base of the sequence, approximately at right angles, to compensate for the unfavourable residual interactions. Again, depending on the decay paths, the bandheads might, or might not, be isomeric. This is the mechanism proposed in Ref. [159] for the formation of the $32 \mu$ s isomer in ${ }^{189} \mathrm{~Pb}$.

4.1.2. Octupole-vibration mixing Coupling of the many-particle configurations to the octupole vibration is a major factor in the formation of favoured configurations in heavy nuclei. Octupole correlations become important when there are pairs of single-particle orbitals near the Fermi surface, which are related by $\Delta j^{\pi}=3^{-}$. Examples are the $i_{13 / 2} / f_{7 / 2}$ proton orbitals and the $j_{15 / 2} / g_{9 / 2}$ neutron orbitals above the $Z=82$ and $N=126$ shell gaps, respectively. As well as contributing to the energy lowering discussed above, the favoured configurations are often those which are related by a spin/parity difference of $3^{-}$, leading to strong E3 transitions and isomers (because of the high multipole order) in the formation of the yrast line.

The important point is that such E3 transitions can be used as indicators of specific configuration changes and relationships. In the first instance, "spin-flip" transitions corresponding to an orbital change of either $\nu j_{15 / 2} \rightarrow \nu i_{11 / 2}$ or $\pi i_{13 / 2} \rightarrow$ $\pi h_{9 / 2}$, are known as type- $B$ in the categorization of Ref. [160], for which an $E 3$ strength of $3-5$ W.u. is expected. Type- $A$ transitions are an order of magnitude stronger, $\sim 20$ W.u. and often larger, and can usually be associated with the $\nu j_{15 / 2}$ $\rightarrow \nu g_{9 / 2}$ or $\pi i_{13 / 2} \rightarrow \pi f_{7 / 2}$ configuration changes. As shown in detail in a number of publications [161, 162, 163, 164], even larger enhancements are observed due to constructive interference in cases involving both proton and neutron admixtures and configuration changes.

This phenomenon turns out to be a dominant factor in the formation of high-spin isomers in the radon region and implies configuration constraints on both initial and final states. It should be noted that at the time of the review of de Voigt et al. [36] this particular aspect was overlooked in the assignment of configurations to high-spin isomers in the radon region, as will be discussed (Sect. 4.1.8).

4.1.3. Valence configurations: the example of ${ }^{212} \mathrm{Po}_{\mathrm{o}}$ The level scheme of the nucleus ${ }^{212} \mathrm{Po}$, which has two neutrons and two protons outside a ${ }^{208} \mathrm{~Pb}$ core, provides an 
archetype of the structures expected where there are equal numbers of valence protons and neutrons. Multiplets up to spin 18 are associated with $j^{2}$ and $\pi\left(j_{1}^{2}\right) \nu\left(j_{1} j_{2}\right)$ configurations [165].

Successive alignment of particles leads to energy sequences which roughly follow inverted parabolas. The first manifestation is an isomer at spin 8, mainly from alignment of the $g_{9 / 2}^{2}$ neutrons. Initially it was proposed that the long-lived, $\alpha$-decaying isomer in ${ }^{212} \mathrm{Po}$ arose from the $16^{+}$state formed by adding a neutron $g_{9 / 2}^{2}$ excitation to the proton $h_{11 / 2}^{2}, 8^{+}$structure. An $18^{+}$alternative has also been suggested arising from the $\pi h_{9 / 2}^{2}, \nu i_{11 / 2}^{2}$ configuration, but it is estimated to be about $800 \mathrm{keV}$ higher. Instead, the current consensus favours an $18^{+}$assignment from the addition of the $\nu\left[g_{9 / 2}, i_{11 / 2}\right] 10^{+}$ excitation to the $8^{+}$state from the $h_{11 / 2}^{2}$ protons [165].

This combination of configurations leads to a number of the states above the $8^{+}$ isomer. The state of maximum spin, $J^{\pi}=18^{+}$, falls lower in energy than a simple sum of the $8^{+}$and $10^{+}$components because of the strongly attractive proton-neutron interaction, culminating in the $45 \mathrm{~s}$ isomer. Its energy is so low that $\gamma$ decays to lower states in ${ }^{212} \mathrm{Po}$ are severely inhibited, forcing the state to $\alpha$ decay. Largely because of this absence of electromagnetic decays, the isomer is still lacking a definitive experimental spin assignment. $J^{\pi}=18^{+}$is preferred since if the state had $J^{\pi}=16^{+}$, in principle there would be a decay path to the proposed $14^{+}$state below [165], but this has not been observed. A weak $(36 \pm 14 \mathrm{keV}) E 4$ branch to the $14^{+}$state has been observed by Kudo et al. [166] with a relative total intensity of about $0.07 \%$ compared to the dominant $\alpha$ branch to ${ }^{208} \mathrm{~Pb}$, with a roughly determined strength about 1 W.u. A similar branch is seen in the decay of the $25 / 2^{+}$isomer in ${ }^{211} \mathrm{Po}$, the main issue in ${ }^{212} \mathrm{Po}$ being the uncertainty in the transition energy which leads to a large uncertainty in the internal conversion coefficient and therefore the $\gamma$-ray transition strength.

\subsubsection{Valence particles for $N \geq 126$ and predicted isomers in the $N=128$ chain}

The nuclei obtained by adding a few particles to ${ }^{208} \mathrm{~Pb}$ are both sources of key residual interactions and important tests of the shell model. However, they also present experimental challenges, since none is accessible with conventional high-spin reactions and the likelihood of long-lived isomers can either help or hinder their study. Complementary but sometimes only incremental advances have been made in this region using incomplete-fusion reactions for ${ }^{210} \mathrm{Po},{ }^{211} \mathrm{Po},{ }^{212} \mathrm{Po}$ and ${ }^{213} \mathrm{At}[165,167,168,169]$, deep-inelastic reactions for ${ }^{210} \mathrm{~Pb},{ }^{211} \mathrm{Bi}$ and ${ }^{211} \mathrm{Po}$ [169, 170, 171], fusion-evaporation reactions with radioactive beams such as ${ }^{8} \mathrm{He}$ for ${ }^{212} \mathrm{Po}$ [172], as well as particle-transfer reactions with oxygen beams $[173,174,175,176]$, which have identified a number of states in the ${ }^{211-214}$ Po isotopes including an $J^{\pi}=8^{+}$isomer in ${ }^{214} \mathrm{Po}$.

As a specific example of the expectations, figure 15 shows the results of calculations (based on those of Refs. $[165,169,177]$ ) aimed at predicting the yrast sequence in the $\pi \nu^{2}, \pi^{2} \nu^{2}$ and $\pi^{3} \nu^{2}$ nuclei (the $N=128$ isotones) ${ }^{211} \mathrm{Bi},{ }^{212} \mathrm{Po}$ and ${ }^{213} \mathrm{At}$. In the case of ${ }^{212} \mathrm{Po}$, although not yet identified, higher-spin states above the $J^{\pi}=18^{+}$isomer are predicted to be formed at the yrast line, and therefore should be preferentially 
populated. These are obtained by adding the (high- $j) \nu\left[g_{9 / 2}, i_{11 / 2}\right]_{10^{+}}, \nu\left[g_{9 / 2}, j_{15 / 2}\right]_{12^{-}}$and $\nu\left[i_{11 / 2}, j_{15 / 2}\right]_{13^{-}}$neutron configurations, to the $\pi\left[h_{9 / 2}, i_{13 / 2}\right]_{11^{-}}$building block (table 3 ), giving the $J^{\pi}=21^{-}, 23^{+}, 24^{+}$sequence shown in figure 15 . The precise details of isomeric decays will depend on the transition energies and also on the energies of states which could intercede and provide a pathway for faster branches. Nevertheless, several of these states are very likely to be isomers, depending on the decay multipolarities and configuration changes. The $J^{\pi}=24^{+}$state, for example, would have a fast decay to the $J^{\pi}=23^{+}$state, but a $23^{+} \rightarrow 21^{-}, M 2$ transition, corresponding to the $\nu j_{15 / 2} \rightarrow i_{11 / 2}$ configuration change, would lead to a lifetime of a few ns. More importantly, the decay from the predicted $J^{\pi}=21^{-}$state directly above the $J^{\pi}=18^{+}$state would be via a moderately enhanced $E 3$ of about $310 \mathrm{keV}$ (a $\pi i_{13 / 2} \rightarrow h_{9 / 2}$ type-B E3, see Sect. 4.1.2). This translates to an expected lifetime of more than $150 \mu$ s for the $J^{\pi}=21^{-}$yrast state. Identifying such a long-lived state directly above the very long-lived $J^{\pi}=18^{+}$ isomer would present a significant challenge to experiment, but also a possible means of identification. It should be noted that most $\gamma$-ray techniques in current use would fail to observe such a state.

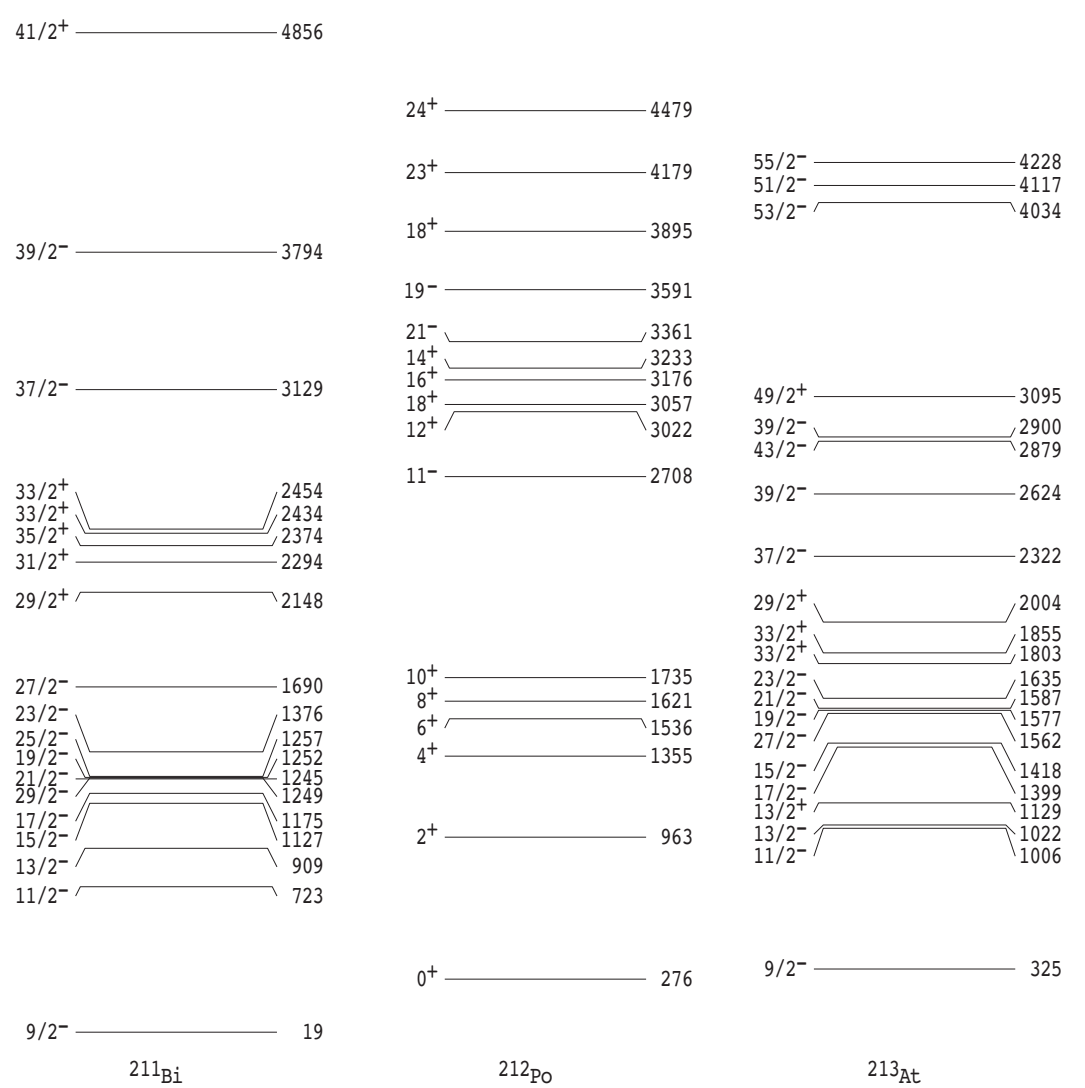

Figure 15. Predicted near-yrast states in the $N=128$ chain (see Refs. $[165,169,177]$ ).

Prediction of these specific properties depends on the knowledge and reliability of the energies of other states, such as the $J^{\pi}=19^{-}$level from the $\pi\left[h_{9 / 2}, i_{13 / 2}\right]_{11^{-}} \nu\left[g_{9 / 2}^{2}\right]_{8^{+}}$ configuration. While it is predicted to lie $230 \mathrm{keV}$ above the $J^{\pi}=21^{-}$state, and is 
unlikely to fall below the $J^{\pi}=21^{-}$state, if it did, it would provide a much faster decay path and therefore a shorter-lived state. Further to this point, the typical accuracy and robustness of the $J^{\pi}=18^{+}$configuration assignment for the 45-s, $\alpha$-decaying isomer were discussed by McGoram et al. [168]. They showed from an analysis of the energies of associated states in ${ }^{211} \mathrm{Po}$, that a minor modification of the residual interactions would give a "prediction" of $2937 \mathrm{keV}$ for the $J^{\pi}=18^{+}$isomer in ${ }^{212} \mathrm{Po}$, essentially reproducing experiment. Moreover, Ref. [168] identified a $J^{\pi}=43 / 2^{+}, 4 \mu$ s isomer decaying via an enhanced $E 3$ transition to a $J^{\pi}=37 / 2^{-}$state in ${ }^{211} \mathrm{Po}$, a pair of states that are related (by a core excitation and coupling to a $p_{1 / 2}$ neutron hole) to the predicted $J^{\pi}=21^{-}$ and $18^{+}$states in ${ }^{212} \mathrm{Po}$. However, the observed energy gap between them was $509 \mathrm{keV}$, rather than the $310 \mathrm{keV}$ predicted by the ESM. If a similar discrepancy applied in ${ }^{212} \mathrm{Po}$, this would lead to an E3 isomer of a few (rather than several hundred) microseconds.

Returning to the predictions for $N=128$ isotones, even the relatively simple nucleus ${ }^{211} \mathrm{Bi}$ has significant experimental uncertainties, as discussed in Refs. [169, 171]. The major issue is that the predicted low-lying $J^{\pi}=29 / 2^{-}$state (the analogue of the $J^{\pi}=18^{+}$isomer in ${ }^{212}$ Po with an $h_{9 / 2}$ proton removed) does not seem to correspond to the observed $1.4 \mu \mathrm{s}$ state at $1257 \mathrm{keV}$, whose properties instead suggest a $J^{\pi}=25 / 2^{-}$ assignment. The observed sequence of states above is also consistent with the predicted set of states (excitation energies and the lack of long lifetimes) decaying eventually to the $J^{\pi}=25 / 2^{-}$state that arises from the $\pi h_{9 / 2} \nu g_{9 / 2}^{2}$ configuration. The $J^{\pi}=29 / 2^{-}$ and $25 / 2^{-}$states are calculated to lie within a few $\mathrm{keV}$ of each other but there is no evidence, for example, for a possible $29 / 2^{-} \rightarrow 25 / 2^{-}$, E2 transition that could occur if the $J^{\pi}=29 / 2^{-}$state were actually about $100 \mathrm{keV}$ higher, i.e. within the theoretical uncertainty. To complicate the issue, a (missed) low-lying $J^{\pi}=29 / 2^{-}$state would be expected to be strongly populated and, in the absence of other decay paths, to either $\beta$ decay or $\alpha$ decay, the latter being the case for the $J^{\pi}=18^{+}$isomer in ${ }^{212}$ Po. Searches for $\alpha$ decays have not been successful, although their sensitivity will depend on the initial population and assumed lifetimes. An equivalent $J^{\pi}=18^{-}$isomer predicted from shell-model calculations for the aligned $\pi h_{9 / 2} \nu\left[g_{9 / 2}^{2}, i_{11 / 2}\right]$ configuration [178] has recently been observed in ${ }^{212} \mathrm{Bi}$ at $1478(30) \mathrm{keV}$ in storage ring measurements [179]. While electromagnetic decays are not observed in the storage ring and the spin and parity is assumed, the argument is made that the observed half-life of $>30 \mathrm{~min}$. compared to the value of 7.0(3) min. for a neutral atom implies that substantial internal conversion would occur when atomic electrons were present. Clearly these two cases are related, but both require more direct spectroscopic information to resolve what is presently a significant issue.

The experimental scheme for the three-proton case of ${ }^{213} \mathrm{At}$ is more extensive but has residual issues because of the likely presence of unobserved low-energy transitions [169]. Four isomers are established including a proposed $J^{\pi}=49 / 2^{+}$isomeric state with a half-life of 45(4) $\mu$ s placed at about $3161 \mathrm{keV}$ and associated with the predicted state at $3095 \mathrm{keV}$ in figure 15. It decays with an enhanced $E 3$ transition to a $J^{\pi}=43 / 2^{-}$state at $2855 \mathrm{keV}$ [169]. The transition energy of $306 \mathrm{keV}$ should be compared with the predicted 
Table 4. Shape-coexisting two-(quasi)particle states in ${ }^{188} \mathrm{~Pb}$.

\begin{tabular}{ccccc}
\hline$I^{\pi}$ & $T_{1 / 2}$ & $E^{*}(\mathrm{keV})$ & configuration & shape \\
\hline $8^{-}$ & $1.2 \mu \mathrm{s}$ & 2577 & $\nu 9 / 2^{+}[624], 7 / 2^{-}[514]$ & high- $K$ prolate \\
$11^{-}$ & $38 \mathrm{~ns}$ & 2702 & $\pi 9 / 2^{-}[505], 13 / 2^{+}[606]$ & high- $K$ oblate \\
$12^{+}$ & $136 \mathrm{~ns}$ & 2710 & $\nu i_{13 / 2}^{-2}$ & spherical \\
\hline
\end{tabular}

spacing of $216 \mathrm{keV}$ between the states arising from the $\left[\pi h_{9 / 2}^{2} i_{13 / 2}, \nu g_{9 / 2} i_{11 / 2}\right]_{49 / 2^{+}}$and $\left[\pi h_{9 / 2}^{2} f_{7 / 2}, \nu g_{9 / 2} i_{11 / 2}\right]_{43 / 2^{-}}$configurations. The $E 3$ decay corresponds to a $\pi i_{13 / 2} \rightarrow f_{7 / 2}$ type-A transition with the $J^{\pi}=18^{+},{ }^{212}$ Po core as a spectator.

4.1.5. Isomerism and shape coexistence in the neutron-deficient $\mathrm{Pb}$ region The neutron-deficient lead region, especially in the middle of the $N=82-126$ shell, is well known for its manifestation of shape coexistence [38], with examples of competition between spherical, prolate and oblate shapes in individual nuclei. Archetypical nuclides include ${ }^{186} \mathrm{~Pb}[180]$ and ${ }^{188} \mathrm{~Pb}[70]$. In the former case, the three coexisting $0^{+}$states may be subject to significant mixing [181]. However, in ${ }^{188} \mathrm{~Pb}$, high-spin isomerism enables clear differentiation to be made between the competing structures, and subsequent magnetic moment measurements [182] have provided supporting evidence. Spin, energy, half-life and configuration details are given in table 4 . It is notable that there are three high-spin, shape-coexisting states in an energy interval of less than $150 \mathrm{keV}$. (As discussed in Sect. 3.1, isomers based on the $K^{\pi}=8^{-}$prolate configuration have been identified in all $N=106$ even-even isotones, from ${ }^{188} \mathrm{~Pb}$ to ${ }^{174} \mathrm{Er}$. Isomers based on the $J^{\pi}=12^{+}$spherical configuration are known from ${ }^{188} \mathrm{~Pb}$ to ${ }^{200} \mathrm{~Pb}$ [44].)

For a comprehensive discussion of this complex and fascinating region of shape coexistence, the reader is referred to the review of Heyde and Wood [38]. In addition, the possibility of finding high- $K$ superdeformed isomers in this mass region has recently been discussed by Shi et al. [105] (see also Sect. 5.3); and a new theoretical description of shape coexistence, through the use of angular-momentum-conserved potential-energy surfaces, has been given by Jiao et al. [183], including predictions of high- $K$ oblate states. A further structural feature associated with this mass region, where isomeric states can provide useful information, is the generation of angular momentum by the shears mechanism - see Sect. 4.1.1.

4.1.6. Particle and hole nuclei neighbouring $Z=82$ Considering the four two-valenceparticle and hole nuclei in which the high-spin $j^{2}$ isomers are expected, ${ }^{206} \mathrm{Hg},{ }^{206} \mathrm{~Pb}$, ${ }^{210} \mathrm{~Pb}$ and ${ }^{210} \mathrm{Po}$, the two-proton-hole case, ${ }^{206} \mathrm{Hg}$, was the only one for which such experimental information was missing, largely because of the absence of experimental techniques to populate high-spin states in neutron-rich nuclei. Observation of the $10^{+}$, $92(8)$ ns isomer [184] then gave an effective charge of $1.60(7)$ for the $h_{11 / 2}$ protonhole orbital. Identification of the isomer was possible through the use of deep-inelastic 
reactions and the associated sensitivity available through the isomeric nature of the state. Recently, several isomers in the four-proton-hole nucleus ${ }^{204} \mathrm{Pt}$ have been identified in a fragmentation reaction [185]. One of these is tentatively assigned as the analogous $10^{+}$state. It would represent the lowest- $Z, N=126$ isotone to have its $j^{2}$ isomer identified, although modification of the two-body matrix elements was necessary to reproduce the observed spectrum of states.

Cases where significant information on isomers has now been obtained leading to more accurate effective nucleon-nucleon interactions include ${ }^{203} \mathrm{Hg}$ [152], ${ }^{204} \mathrm{Hg}$ [186], ${ }^{206} \mathrm{Hg}$ [184], and ${ }^{208} \mathrm{Hg}$ [187], ${ }^{204} \mathrm{Tl}$ [188], ${ }^{205} \mathrm{Tl}$ [189], and ${ }^{209} \mathrm{Tl}$ [187], ${ }^{210} \mathrm{~Pb}$ [171] and ${ }^{211} \mathrm{~Pb}$ [190], as well as the $\pi \nu^{-3}$ isotope, ${ }^{206} \mathrm{Bi}$ [191]. The ${ }^{206} \mathrm{Bi}$ scheme extends above $10 \mathrm{MeV}$ and includes two high-lying isomers $\left(J^{\pi}=31^{+}\right.$and $\left.28^{-}\right)$connected by an E3 transition of relatively low strength $(<0.4$ W.u. $)$. Shell-model calculations produce excellent agreement with the states up to $J^{\pi}=23^{+}(7.2 \mathrm{MeV})$, the maximum spin obtained from the $\pi i_{13 / 2}, \nu i_{13 / 2}^{-3}$ configuration composed of the aligned valence particles and holes. The higher states up to the $J^{\pi}=31^{+}$isomer are presumed [191] to involve either proton or neutron core excitations (or both), but those were not within the scope of the shell-model calculations reported.

The two-proton-hole, two-neutron-hole nucleus ${ }^{204} \mathrm{Hg}$ [186], includes a proposed $J^{\pi}=22^{+}$isomer at $7226 \mathrm{keV}$ obtained from the maximum alignment of the high-spin proton and neutron holes, $\pi h_{11 / 2}^{-2}, \nu i_{13 / 2}^{-2}$, predicted from a shell-model calculation to lie at $7262 \mathrm{keV}$. Similar agreement (within $\leq 50 \mathrm{keV}$ ) is found for essentially all observed and predicted states above $\sim 9 \hbar$. Considering further a very simple approach, the $\nu i_{13 / 2}^{-2}, J^{\pi}=12^{+}$state is known at $4027 \mathrm{keV}$ in ${ }^{206} \mathrm{~Pb}$ and the two-proton-hole $\pi h_{11 / 2}^{-2}$, $J^{\pi}=10^{+}$state is found at $3723 \mathrm{keV}$ in ${ }^{206} \mathrm{Hg}$. Combining the experimental energies of these two states and allowing for the residual interaction of $-543 \mathrm{keV}$ predicted for the fully aligned coupling, leads to an expected energy of $4027+3723-543=7207 \mathrm{keV}$, very close to both the observed state and the shell-model calculation (which effectively incorporates the same interactions).

The lifetime limit of $>700 \mathrm{~ns}$ for the $921 \mathrm{keV}$ decay to a $J^{\pi}=19^{-}$state corresponds to a relatively slow $E 3$ transition of $<2 \mathrm{~W}$.u., consistent with two alternative configurations for the yrast $J^{\pi}=19^{-}$state. Either would involve a $j$-forbidden $E 3$ corresponding to a $\pi h_{11 / 2}^{-1} \rightarrow d_{3 / 2}^{-1}$ configuration change in one case, or to a $\nu i_{13 / 2}^{-1} \rightarrow f_{5 / 2}^{-1}$ change in the other. These should be compared with the more enhanced E3 transitions outlined in Sect. 4.1.2 and observed in the radon region (Sect. 4.1.8). Note also that, in the ${ }^{204} \mathrm{Hg}$ case, the $J^{\pi}=10^{+}$state from the $h_{11 / 2}^{-2}$ configuration does not compete with more complicated configurations that involve contributions from both proton and neutron holes, such as the yrast $J^{\pi}=11^{-}$state.

4.1.7. Neutron-rich $\mathrm{Hg}$ and $\mathrm{Pb}$ cases In pioneering work carried out over a decade ago, Pfützner et al. [192] identified an isomer with $\mathrm{T}_{1 / 2}=5 \mu \mathrm{s}$ in ${ }^{212} \mathrm{~Pb}$, four neutrons beyond the stable isotope, ${ }^{208} \mathrm{~Pb}$, demonstrating the potential of fragmentation reactions for populating heavy neutron-rich nuclei (see Sect. 10.2.1). The isomer was tentatively 
associated with the expected seniority isomer from the neutron $g_{9 / 2}^{2}$ configuration, but a detailed scheme was not established. Recently, Gottardo et al. [193] have used similar reactions to revisit the case of ${ }^{212} \mathrm{~Pb}$ and to identify isomers in the more neutronrich, even-even isotopes, ${ }^{214-216} \mathrm{~Pb}$. They do not, however, identify the low-energy E2 transitions that connect the proposed $J_{\max }$ and $J_{\max }-2$ states of the multiplet. This is a recurring challenge in the spectroscopy of heavy nuclei where internal conversion dominates over $\gamma$ decay at low energies. Nevertheless, within these current limitations, the results have been used as a test of shell-model interactions with increasing neutron number at $Z=82$, leading to a claim of evidence for the influence of three-body forces, based on the absolute value and neutron-number dependence of the effective charge. This is re-iterated in the recent study by the same authors of the (proposed) $8^{+}$isomeric state in the neutron-rich isotone ${ }^{210} \mathrm{Hg}$ [194].

4.1.8. Multiparticle core-excited isomers: ${ }^{212} \mathrm{Rn}$ and the radon region With four valence protons, the maximum angular momentum that can be obtained at reasonably low energies in the radon nuclei is $J^{\pi}=20^{+}$from the $\pi\left[h_{9 / 2}^{2}, i_{13 / 2}^{2}\right]$ configuration. (The $\pi\left[h_{9 / 2}, i_{13 / 2}^{3}\right]_{21^{-}}$configuration is not competitive in energy.) Neutrons can be promoted out of the core so as to gain angular momentum by populating the high- $j$ orbitals above the neutron Fermi surface, the energy cost being partly offset by the strong residual proton-neutron interactions. In ${ }^{212} \mathrm{Rn}$, for example, states with higher spin can be formed by combining the valence protons and neutron-core excitations into the $g_{9 / 2}, i_{11 / 2}$, and $j_{15 / 2}$ orbitals (see table 3 ). The lowest of these are likely to involve coupling of the low-lying $\pi\left[h_{9 / 2}^{3}, i_{13 / 2}\right]_{17^{-}}$and $\pi\left[h_{9 / 2}^{2}, i_{13 / 2}^{2}\right]_{20^{+}}$components to double neutron-core excitations, with two $p_{1 / 2}$ holes coupled to zero, such as $\left[p_{1 / 2}^{-2}, g_{9 / 2}, i_{11 / 2}\right]_{10^{+}}$ and $\left[p_{1 / 2}^{-2}, j_{15 / 2}^{2}\right]_{14^{+}}$giving states with $J \leq 34 \hbar$. Beyond, triple neutron-core excitations also become competitive, with the maximum spins available from energetically favoured configurations being about $40 \hbar$. This spin and energy region has been probed in the most recent studies $[195,196]$, which include the highest-spin isomers so far identified.

Much of this programme of study began with the discovery of high-spin isomers in the $N=126$ isotope ${ }^{212} \operatorname{Rn}[197,198]$. At that time, the energy of the $8^{+} \rightarrow 6^{+}$transition was uncertain, but it was later measured as $54.2 \mathrm{keV}$ by Stuchbery et al. [199]. A $J^{\pi}$ $=22^{+}$isomer was originally placed by Horn et al. [197] at an energy of $6167.3+\Delta \mathrm{keV}$, and assumed to connect to the $J^{\pi}=20^{+}$state obtained from the alignment of spins of the valence protons by an unobserved low-energy transition, partly on the basis of ESM calculations that predicted high-spin core-excited isomers to intercede in this region of the yrast line. The $J^{\pi}=22^{+}$isomer was itself populated through a cascade of isomers with $J^{\pi}=25^{-}, 27^{-}$and $30^{+}$. In the recent comprehensive measurements of Ref. [196], an extension of the study which identified a $J^{\pi}=33^{-}$isomer feeding the $30^{+}$state via an enhanced E3 transition [163], the original assumptions of Refs. [197, 198] have been vindicated, with the definition (through the energies of new crossover transitions) of the gap $\Delta=7.6 \mathrm{keV}$. The level scheme was also extended to excitation energies above 13 $\mathrm{MeV}$, including six new isomers, the highest with $J^{\pi}=\left(38^{+}\right)$at $12.547 \mathrm{MeV}$, arising 
from triple neutron-core excitations.

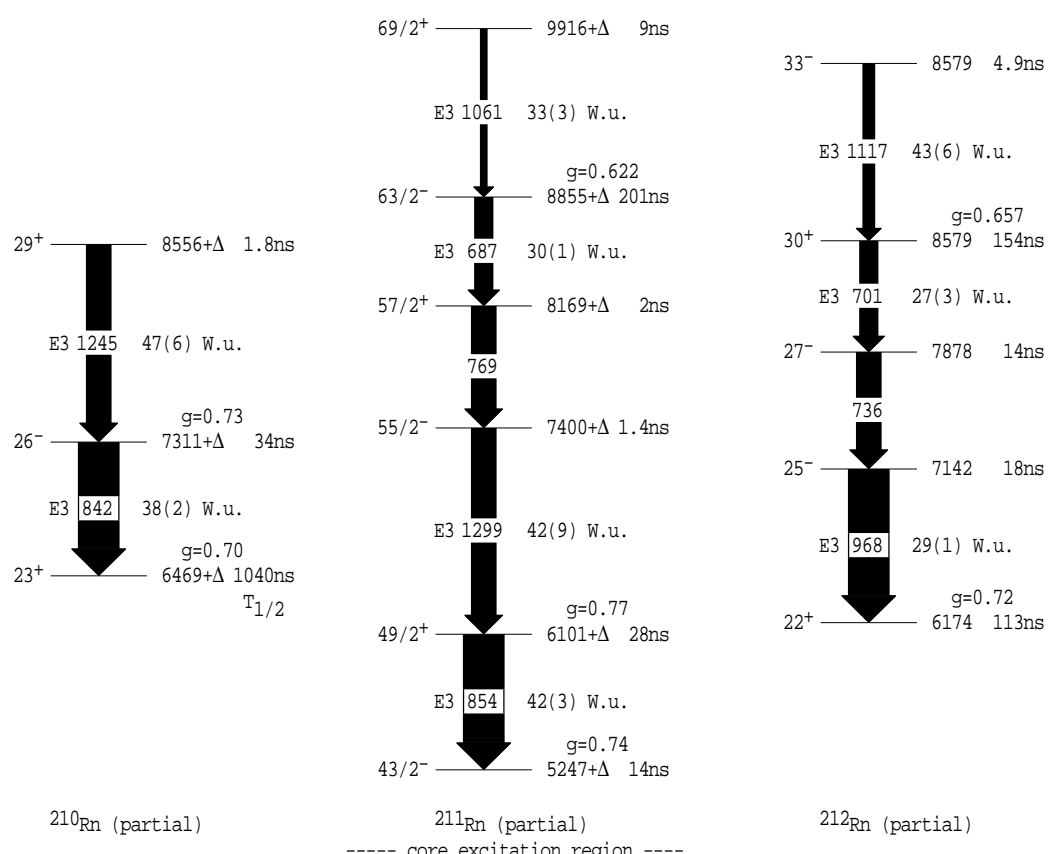

Figure 16. Partial level schemes for the radon neighbours in the core-excited region, with half-lives, measured $E 3$ strengths and $g$-factors.

Figure 16 shows partial schemes of the main yrast states in the region of core excitations in ${ }^{210} \mathrm{Rn}_{124},{ }^{211} \mathrm{Rn}_{125}$ and ${ }^{212} \mathrm{Rn}_{126}$. Most of the yrast states are isomers, but the striking feature is the presence of strongly enhanced $E 3$ transitions connecting pairs of states, such as the $J^{\pi}=30^{+}$and $27^{-}$levels in ${ }^{212} \mathrm{Rn}$, and the $J^{\pi}=63 / 2^{-}$and $57 / 2^{+}$ levels in ${ }^{211} \mathrm{Rn}$. Whatever the structure of each of the upper states, the state to which each decays must therefore be closely related. Their measured $g$-factors are relatively low and approximately given by $g \approx\left(1.1 J_{\pi}-0.1 J_{\nu}\right) / J$, consistent with core-excited configurations where about $40 \%$ of the angular momentum is provided by the high-spin neutron populated by the core excitation.

A compilation of the properties of high-spin isomers decaying by $E 3$ transitions in the At, Rn and Fr isotopes was reported some years ago in Ref. [200] and compared with the predictions of the Multiple Octupole Coupling (MOPC) model, with good overall agreement for the 15 cases known at that time. This agreement with experiment included excitation energies, $E 3$ strengths and $g$-factors, the last of which are largely controlled by the number of protons and neutrons in the configuration.

The background to the understanding that has been reached can be gleaned from the approximate wave-function configurations assigned to the $33^{-} \rightarrow 30^{+} \rightarrow 27^{-}$sequence in ${ }^{212} \mathrm{Rn}$ that includes a mixed configuration for the intermediate $30^{+}$state:

$33^{-}: \pi\left[h_{9 / 2}^{2}, i_{13 / 2}^{2}\right]_{20^{+}} \nu\left[p_{1 / 2}^{-2}, i_{11 / 2}, j_{15 / 2}\right]_{13^{-}}$;

$30^{+}: \alpha\left\{\pi\left[h_{9 / 2}^{2}, i_{13 / 2}^{2}\right]_{20^{+}} \nu\left[p_{1 / 2}^{-2}, g_{9 / 2}, i_{11 / 2}\right]_{10^{+}}\right\}+\beta\left\{\pi\left[h_{9 / 2}^{3}, i_{13 / 2}\right]_{17^{-}} \nu\left[p_{1 / 2}^{-2}, i_{11 / 2}, j_{15 / 2}\right]_{13^{-}}\right\}$;

$27^{-}: \pi\left[h_{9 / 2}^{3}, i_{13 / 2}\right]_{17^{-}} \nu\left[p_{1 / 2}^{-2}, g_{9 / 2}, i_{11 / 2}\right]_{10^{+}}$. 
The $33^{-} \rightarrow 30^{+}$E3 transition can proceed by either the $j_{15 / 2} \rightarrow g_{9 / 2}$ (type-A) transition to the first component of the $J^{\pi}=30^{+}$state wave function, or by $i_{13 / 2} \rightarrow h_{9 / 2}$ (type-B) transition to the second, with the possibility of constructive interference $[162,163,196]$. The $30^{+} \rightarrow 27^{-}$decay is similar, with two interfering components.

A similar scenario applies to the decay of numerous high-spin isomers with related configurations that have been discovered in this region, including the astatine isotopes [151, 169, 201, 202], other radon isotopes [203, 204] and the francium isotopes [205, 206, 207].

4.1.9. DIPM model of ${ }^{212}$ Rn The DIPM calculations of Andersson et al. [139] used the Strutinsky method and the modified harmonic oscillator potential to predict states (specifically yrast traps) up to $30 \hbar$ in ${ }^{212} \mathrm{Rn}$. In order to match the spherical singleparticle levels for the $i_{13 / 2}$ proton and $j_{15 / 2}$ neutron, they adjusted the parameters given by Nilsson et al. [208] to displace those levels through a reduction of the Nilsson parameter $\mu$, hence the label of displaced modified oscillator, or DMO. Pairing was not included. The calculations of Matsuyanagi et al. [141] were similar in approach but included blocked BCS pairing and a deformed axially-symmetric Woods-Saxon potential. The energies of some orbitals were again shifted to match spherical cases. The deformation was determined for each configuration. As well as analyzing yrast isomers up to spin $30 \hbar$ to compare with the experimental states known at the time, they also predicted a $J^{\pi}=35^{-}$yrast trap. Applications to ${ }^{212} \mathrm{Rn}$ and ${ }^{213} \mathrm{Rn}$ using essentially the same approach were also reported in Ref. [209].

More extensive calculations for ${ }^{212} \mathrm{Rn}$ have been reported recently [196]. These use the configuration-constrained approach (see $[80,114]$ ) with a non-axial WoodsSaxon potential, universal parameters [210], and the Lund convention for specifying the deformation [137]. For most configurations the minimum is found to be close to $\gamma=$ $60^{\circ}$ corresponding to oblate deformation, and no deformed solution was found for cases involving only valence protons, such as the $J^{\pi}=17^{-}$and $20^{+}$yrast states. In common with earlier calculations, the magnitude of predicted deformation is generally $|\beta| \leq 0.10$, even when extended to configurations involving triple neutron-core excitations.

As outlined earlier, the historical development of the understanding of the structure of high-spin isomers in ${ }^{212} \mathrm{Rn}$ initially involved a shell-model perspective and then models incorporating (oblate) deformation. While there were common assignments for many configurations, different configurations were suggested for a number of the key states. At the time of the review of de Voigt et al. [36] the multiparticle configurations based on the predictions of the DIPM were mostly in agreement with each other, and generally accepted, although there remained some differences between the results.

However, a number of those assignments were questioned, both before that review and after (see for example Refs. $[162,163,211]$ ) since they were incompatible with the experimental feature of enhanced $E 3$ transitions between states such as the $J^{\pi}=30^{+}$ and $27^{-}$pair in ${ }^{212} \mathrm{Rn}$.

In ${ }^{212} \mathrm{Rn}$, for example, the DIPM and DMO assignments for the $J^{\pi}=30^{+}$and $27^{-}$ 


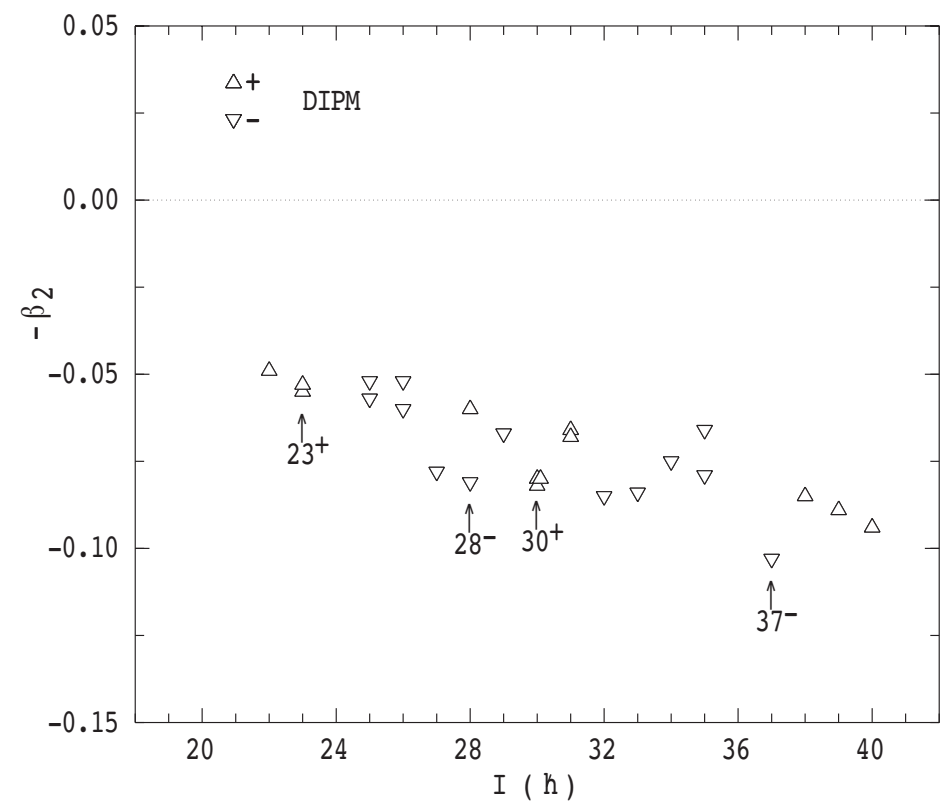

Figure 17. Equilibrium quadrupole deformations for ${ }^{212} \mathrm{Rn}$ from the DIPM model, from Ref. [196]. (Note that all have small values of $\beta_{4}$ and $\gamma \approx 60^{\circ}$.) The arrows mark states that have a common $J^{\pi}=18^{-}$proton component as discussed in the text. Note also that the calculations use the Lund convention, hence for $\gamma=60^{\circ}$, the $\beta_{2}$ parameter is of opposite sign to $\beta$ or $\epsilon_{2}$ in the other DIPM calculations discussed in the text.

states in ${ }^{212} \mathrm{Rn}$ were associated with the configurations:

$30^{+}: \quad \pi\left[h_{9 / 2}^{2}, f_{7 / 2}, i_{13 / 2}\right]_{18^{-}} \nu\left[p_{1 / 2}^{-2}, g_{9 / 2}, j_{15 / 2}\right]_{12^{-}} ; \quad \beta \sim-0.095\left(\epsilon_{2}=-0.10\right) ;$

$27^{-}: \quad \pi\left[h_{9 / 2}^{3}, i_{13 / 2}\right]_{17^{-}} \nu\left[p_{1 / 2}^{-2}, g_{9 / 2}, i_{11 / 2}\right]_{10^{+}} ; \quad \beta \sim-0.070\left(\epsilon_{2}=-0.08\right)$.

From simple angular-momentum coupling restrictions and given the number of orbital changes required, an E3 transitions between such configurations are essentially forbidden. The problem is compounded since an enhanced $33^{-} \rightarrow 30^{+}$transition is also observed, as discussed in the previous section, and cannot be explained by DIPM configurations. Similar arguments apply to the $69 / 2^{+} \rightarrow 63 / 2^{-} \rightarrow 57 / 2^{+}$sequence in ${ }^{211} \mathrm{Rn}$ [163].

While the deformation energy in the DIPM accounts for a considerable portion of the shell-model interaction, it is not precisely equivalent, the difference being configuration dependent. (A direct comparison of deformation energy and residual interactions for the configuration proposed for the $J^{\pi}=30^{+}$isomer in ${ }^{212} \mathrm{Rn}$ is given in Ref. [155].) Andersson et al. [139] showed that inclusion of the deformation in their DMO model dramatically improved the comparison between theory and experiment, compared to a pure "spherical" case. However, this improvement in energy should not be taken to imply that deformation is a necessary component, and it can be misleading if the configuration assignments disagree with other experimental properties.

A second problem which confronts the DIPM interpretation is the small quadrupole moment measured for the $J^{\pi}=63 / 2^{-}$isomer in ${ }^{211} \mathrm{Rn}$ [203]. Its magnitude can 
be explained by the mixed shell-model configurations, without requiring significant deformation [203]. A similar conclusion was reached [205, 206] from the measurement of the quadrupole moments in the francium isotopes, which have related configurations, although, in contrast, general agreement is obtained with MOPC shell-model calculations. The theoretical analysis of Sagawa and Arima [212], for a number of high-spin isomers in the lead region, also concurs.

It should be noted that while the magnitude of the deformation predicted by the calculations becomes larger as more neutron core excitations occur, it remains in the region of $\beta \leq 0.10$. The trend can be seen in figure 17, where several states are marked with arrows: each contains the $18^{-}$proton component discussed above which seems to be artificially low in the DIPM calculations, confusing the assignments when only energies are considered [196].

4.1.10. Multi-particle isomers and blocking As multiparticle states become more complex with the addition of more particles, the $E 3$ strengths that are characteristic of specific configuration changes are likely to be modified. Self-consistency becomes an issue since components of the underlying octupole vibration may be blocked, resulting in additional effects on the coupling and the lifetimes. This is illustrated in figure 18 with the chain of isomers observed in the range of astatine isotopes ${ }^{210-213}$ At $[151,201,202]$. The lower strength of the transition in ${ }^{212}$ At corresponding to the proton $i_{13 / 2} \rightarrow f_{7 / 2}$ transition was attributed by Byrne et al. to the additional $i_{11 / 2}$ neutron [202], and a similarly low strength is observed in ${ }^{213}$ At [169]. To make further progress with such studies, however, developments in theory which incorporate the octupole vibration in a microscopic rather than an ad hoc fashion, and that can treat neutron-core excitations, will be necessary.

\subsection{Nuclei near ${ }^{146}$ Gd}

High-spin isomers are known to dominate the yrast structure of many nuclei in the region near ${ }^{146} \mathrm{Gd}$, where spherical shape is stabilised by the $N=82$ shell gap combined with the $Z=64$ sub-shell closure. The properties of nuclei in this region were reviewed by de Voigt et al. [36], but since then many new isomers have been observed in nuclei near $\mathrm{N}=81-85$ and $\mathrm{Z}=60-71$, in parallel with studies in the radon region. The implementation of recoil mass separators in conjunction with heavy-ion fusion-evaporation reactions has allowed a number of long-lived states in proton-rich nuclei to be identified, including isomers in proton-unbound nuclei at the drip line, as discussed in Sect. 10.1.1.

Particularly impressive is the extensive work carried out on the $N=83$ isotones, where a suite low-, medium- and high-spin isomers, formed by coupling of proton $h_{11 / 2}$ and neutron $f_{7 / 2}, h_{9 / 2}$ and $i_{13 / 2}$ orbitals above the shell gaps at $Z=64$ and $N=82$, were identified, as summarized in figure 19. Because of the proximity of $\Delta j=3$, $\Delta l=3$ orbitals near the proton $\left(\pi h_{11 / 2} \leftrightarrow \pi d_{5 / 2}\right)$ and neutron $\left(\nu i_{13 / 2} \leftrightarrow \nu f_{7 / 2}\right)$ Fermi surfaces, octupole excitations are also important in this region. This is manifested by 


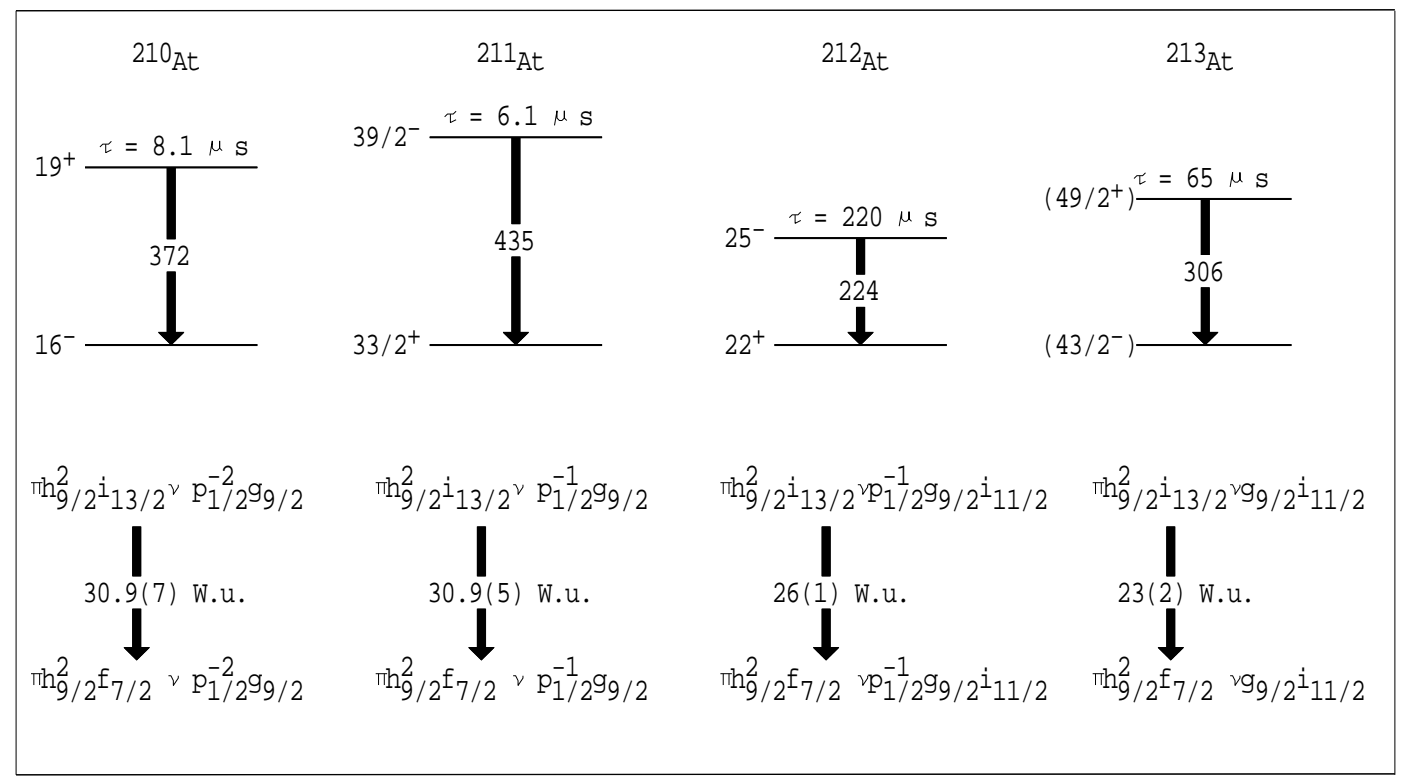

Figure 18. Related isomers in the At isotopes, their mean lives and their $E 3$ decay strengths. The lower part of the figure shows the dominant initial and final state configurations.

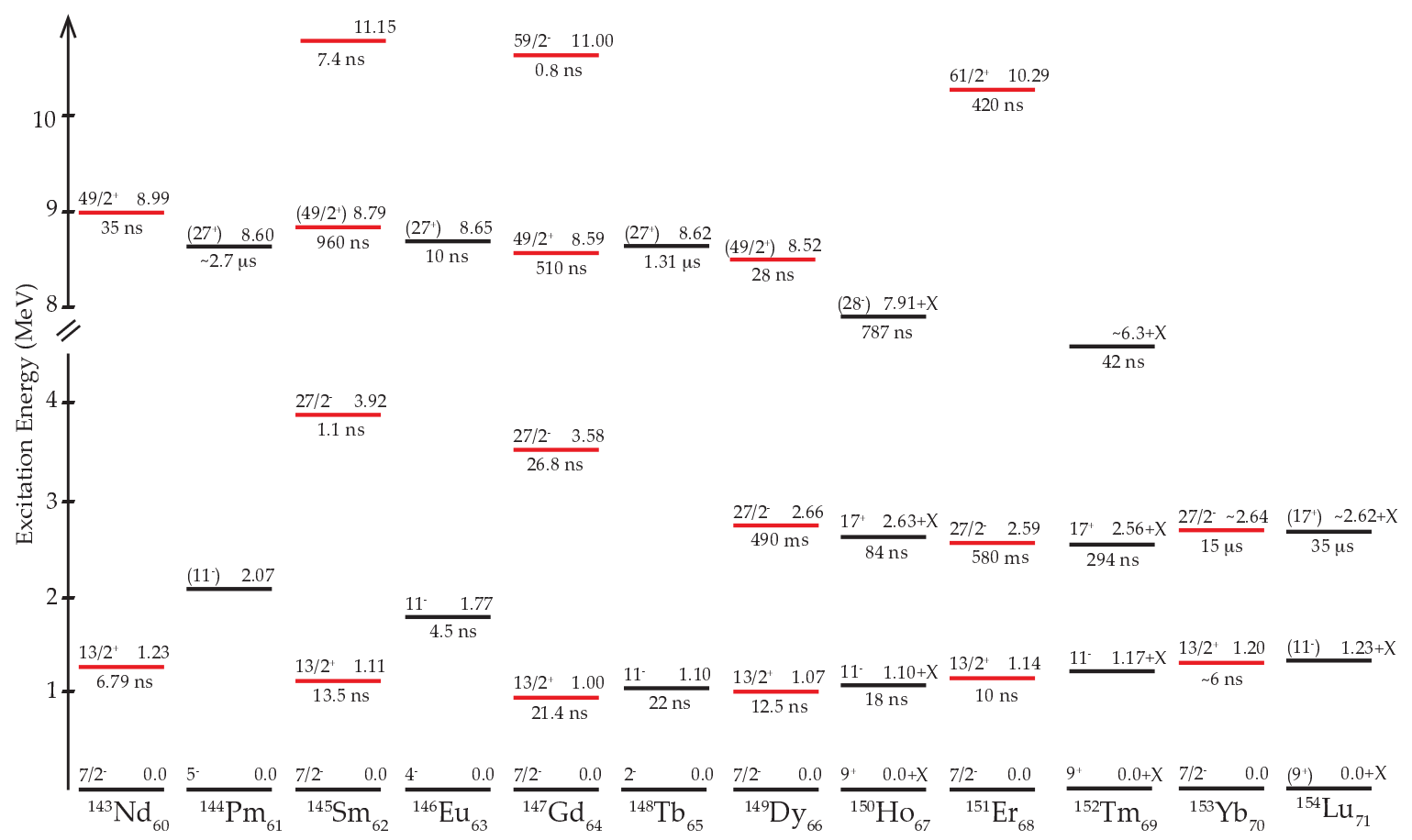

Figure 19. Systematics of isomers in the $N=83$ isotones. The data are from the review of de Voigt at al. [36] together with new results for ${ }^{154} \mathrm{Lu}$ [216], ${ }^{153} \mathrm{Yb}$ [217], ${ }^{152} \operatorname{Tm}[218,219],{ }^{151} \operatorname{Er}[220,221,222,223,224],{ }^{150} \mathrm{Ho}[219,225],{ }^{149}$ Dy [224, 226, 227], ${ }^{148} \mathrm{~Tb}[228],{ }^{147} \mathrm{Gd}[229,230],{ }^{146} \mathrm{Eu}[231,232],{ }^{145} \mathrm{Sm}[233,234,235,236,237]$, ${ }^{144} \mathrm{Pm}[238]$ and ${ }^{143} \mathrm{Nd}[239]$. 


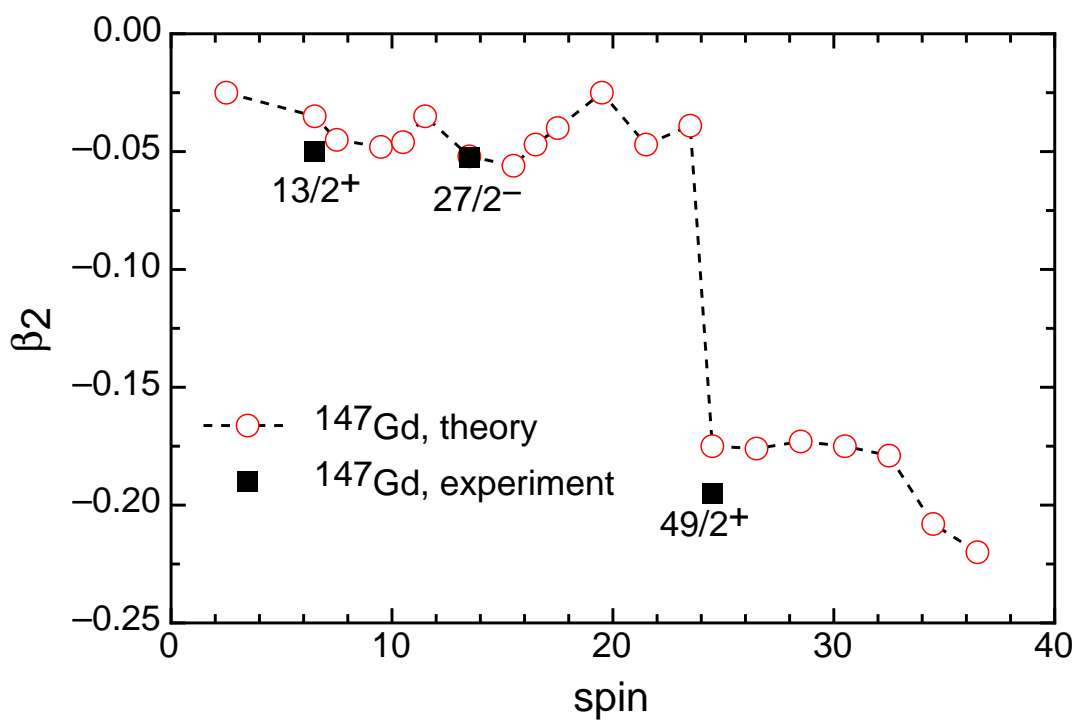

Figure 20. Predicted deformations of multi-particle states in ${ }^{147} \mathrm{Gd}$ [239] compared with the deformations extracted from measurements of quadrupole moments [242].

the observed enhanced $E 3$ strength of $35-40$ W.u. in decays of the $J^{\pi}=13 / 2^{+}$isomers, which are associated with an admixture of the $\nu f_{7 / 2} \otimes 3^{-}$and $\nu i_{13 / 2}$ configurations. Similarly, the $J^{\pi}=11^{-}$isomers in the odd-odd nuclei are assigned the $\left[\pi h_{11 / 2}\right.$ $\left.\nu f_{7 / 2}\right]_{9^{-}} \otimes 3^{-}$configuration. Calculations using the deformation independent particle model (DIPM) [213] and diabatic potential energy surface model (DPES) [214, 215] predict that the $N=83$ isomers have related multi-particle structure. For example, in the medium-spin regime, the $J^{\pi}=27 / 2^{-}$and $17^{+}$isomers are assigned the $\pi\left[h_{11 / 2}^{2}\right]_{10^{+}}$ $\nu f_{7 / 2}$ and $\pi\left[h_{11 / 2}^{3}\right]_{27 / 2^{-}} \nu f_{7 / 2}$ configurations, respectively.

Much of the progress in recent years was in the high-spin domain, where the fullyaligned $J^{\pi}=49 / 2^{+}, \pi\left[h_{11 / 2}^{2}\right]_{10^{+}} \nu\left[f_{7 / 2}, h_{9 / 2}, i_{13 / 2}\right]_{29 / 2^{+}}$and $J^{\pi}=27^{+}, \pi\left[d_{5 / 2}^{-1}, h_{11 / 2}^{2}\right]_{25 / 2^{+}}$ $\nu\left[f_{7 / 2}, h_{9 / 2}, i_{13 / 2}\right]_{29 / 2}+$ configurations are predicted to be yrast, and associated with the observed isomers. Magnetic-moment measurements in ${ }^{149}$ Dy [227] and ${ }^{147} \mathrm{Gd}[240]$ provided direct experimental evidence regarding such an interpretation. Remarkably, both DIPM and DPES predict a sudden shape change at $J^{\pi}=49 / 2^{+}$, where the yrast states are associated with larger oblate deformation of $\beta_{2} \sim-0.20$, compared to the nearly spherical (weakly oblate) shape of the lower-spin structures. An example of the evolution of deformation as a function of angular momentum in ${ }^{147} \mathrm{Gd}$ is shown in figure 20. The measured negative sign for the quadrupole moments for the $J^{\pi}=13 / 2^{+}$, $27 / 2^{-}$and $49 / 2^{+}$isomers in ${ }^{147} \mathrm{Gd}[241]$ and the corresponding values of $|Q|=0.73(7)$, $1.26(8)$ and 3.24(18) $b$ [242] provide direct evidence for such a phenomenon. A similar shape-isomerism interpretation was also invoked in the cases of ${ }^{143} \mathrm{Nd}[239,243]$, ${ }^{145} \mathrm{Sm}[233,234]$ and ${ }^{149} \mathrm{Dy}$ [226]. Furthermore, Odahara et al. [243] argued that the $E 1$ and $E 2$ decays from the $J^{\pi}=49 / 2^{+}$isomers in ${ }^{143} \mathrm{Nd}$ and ${ }^{147} \mathrm{Gd}$, respectively, are more hindered, and this was attributed to differences in deformation between the isomer 
and the final state. It was also noted in Ref. [226] that the observed similar excitation energies of the $J^{\pi}=49 / 2^{+}$and $27^{+}$isomers in the $N=83$ isotones, is indicative of the disappearance of the $Z=64$ sub-shell closure at high spin. The strength of the pairing correlations was also suggested to be surprisingly strong in such high-spin states [215], despite several proton and neutron orbitals being blocked.

\section{Deformed nuclei with $A>190$ : extreme shapes, fission and $K$ hindrance}

In the case of a wave function where the intrinsic and deformation components are separable,

$$
<\psi_{f}|\mathbf{T}| \psi_{i}>=<\chi_{f}|\mathbf{T}| \chi_{i}>\otimes<\beta_{f} \mid \beta_{i}>
$$

and if there is a significant difference between the mean deformations $\beta_{i}$ and $\beta_{f}$ associated within nuclear-potential sub-minima, there could be limited overlap and therefore retarded transitions connecting them, possibly leading to isomers. In practice, such wave functions have a finite extent so that very large differences in deformation with a minimum of fluctuations and a significant barrier would be necessary to cause hindrances at the level of those caused by $K$-forbiddenness, for example. Hence, manifestations in terms of observable lifetimes have been been relatively limited and elusive, the main exception to date being that of fission isomers. These are superdeformed states that are relatively stable at low spin, but also can be formed initially at low spin and low excitation energy, with very limited decay paths.

Stimulated partly by the discovery of high-spin superdeformation in non-fissile nuclei (see for example Ref. [244]) extensive theoretical efforts that now span a broad range of the periodic table, have been made in the last two decades, aimed at understanding both high-spin decays and fission. These have included non-fissile regions, typically using Hartree-Fock BCS or Strutinsky approaches, with and without full explorations over the $(\beta, \gamma)$ deformation plane $[245,246,247,248]$ with a number focussing on important details of the generator-coordinate methods [249, 250, 251]. There are numerous studies of fissile nuclei (for example Refs. [252, 253] and a comprehensive work using the Gogny force that extends into the superheavy region of the nobelium isotopes [254]). It includes enumeration of two-quasiparticle states in both normal and superdeformed wells. Although Ref. [255] previously concluded that fission isomers were unlikely in superheavy nuclei because a second well would be unstable, Delaroche et al. [254] do obtain superdeformed solutions in their study that might become the ground states, although there is significant sensitivity to the treatment of the outer barrier in this and other models. As well, the possibility of superdeformed states in oblate rather than prolate superheavy nuclei $(Z \geq 120)$, with longer-lived high- $K$ isomers, has been raised [256, 257]. Although it is difficult to predict specific decay rates, such a development may open new horizons for the observable limits of the nuclear landscape. Not unexpectedly, the influence of octupole and higher-order shape degrees of freedom will be important for isomers in transuranic nuclei [258, 259]. 
Finally, an extensive study with the macroscopic-microscopic model was also reported recently by Möller et al. [260, 261], covering in detail a large part of the known, and unknown, periodic table.

It should be noted that in the event that such deformed states occur at very low energies, one would be faced with a situation analogous to that of very long-lived superheavy nuclei, where the absence of decay modes could hamper their discovery. In this context, while the results have been greeted with some skepticism, Marinov and colleagues (see for example Refs. [262, 263, 264, 265, 266]) have reported long-lived activities from both induced radioactivity and abundances of rare isotopes in nature that they attribute to the existence of very long-lived isomers produced in secondary minima (superderformed) and tertiary minima (hyperdeformed) in nuclear species ranging from the tungsten region, thorium and other heavy isotopes, and also in superheavy nuclei.

\subsection{Second-well fission isomers}

The pioneering studies in the 1960s of Polikhanov and collaborators [267] led, over the ensuing years, to the unfolding of the "superdeformed" regime in nuclei with $92 \leq Z \leq 97$ [268, 269, 270, 271, 272, 273, 274]. For these atomic numbers, the repulsion between the protons is approximately balanced by the liquid-drop surface tension, leading to an enhanced role for shell effects, whereby a superdeformed shell gap provides a second minimum in the potential-energy surface. With highly elongated shapes, the superdeformed nuclei are susceptible to fission, enabling detection techniques to be applied with sensitivity to isomeric lifetimes down to a few picoseconds.

In more recent years, a rich spectroscopy of rotations and vibrations has been revealed in the superdeformed second well $[273,274]$. Nevertheless, the early indications of two-quasiparticle high- $K$ isomers in the second well of the even-even plutonium and curium isotopes $[269,270]$ have not been followed up by detailed spectroscopy. It is not known if they fission directly, or if they decay by $\gamma$-ray and conversion-electron emission to the corresponding second-well ground state, and then fission. This aspect remains a challenge for the future. However, despite the limited information, it is clear [270, 275] that excited states (presumably of high- $K$ ) in the second well can have substantially longer half-lives than their corresponding second-well minima. This extra stability apparently caused by the $K$ quantum number may have more general significance as the limits to nuclear binding are approached, such as in superheavy nuclei (see Sect. 5.4).

Theoretically, the macroscopic-microscopic shell-correction approach has been very successful in explaining the basic features of fission isomers [269, 270], with improved modelling in recent years $[276,277,278]$. As discussed in Sect. 5.4, the extended halflives of the supposed high- $K$, second-well isomers can be understood qualitatively with the help of configuration-constrained, potential-energy-surface calculations [275], but more work is required on this aspect. 


\subsection{Superdeformed bandheads}

Despite the large difference in shape between states in the superdeformed well compared to the normal states, there are no clear examples in high-spin studies of heavy nuclei where decays between the wells are sufficiently inhibited to result in isomerism. Apparently the mixing between the superdeformed states and the much higher density of states of normal deformation results in substantial tunnelling through the potential barrier between the deformations. The result is that at spins $\sim 10 \hbar$, the $\gamma$-ray cascade down the sequence of superdeformed states producing the "picket-fence" of regularly spaced transitions which identify the superdeformed bands, is funnelled out before the bandhead (presumably an excited $0^{+}$state in the even-even cases) is reached. While this is partly a consequence of the method of population (proceeding from high spin and high excitation energy) rotation is believed to be a key factor in stabilizing the very deformed configurations in non-fissile nuclei [279], hence differentiated low-lying $0^{+}$states might not exist. There may be exceptions to this, for example in the mercury isotopes where the superdeformed bands lie at relatively low energies. Whether such a $0^{+}$bandhead would have a significant lifetime depends on the available decay paths and transition energies. The most likely would be $E 1$ decays to negative-parity states which (because of octupole vibrations) often lie at low energies. Also important would be $E 0$ decay to the ground state, which could be used as a probe of the difference in deformation $[38,280]$. Identification of the low-spin states has been attempted by populating through $\beta$ decay (see for example Ref. [281]) but has not been successful.

\subsection{High-K isomers in superdeformed nuclei}

There remains the possibility that high- $K$ states within the superdeformed well would have additional decay constraints because of lower mixing that could reduce the tunnelling effects and even lead to the existence of high- $K$ isomers. The large deformation can be taken to imply well-defined $K$, but that is still likely to be eroded by mixing because of the high excitation energies. The hope would be to use time correlations to identify very weak paths (given that population of superdeformed structures is already low). This becomes an experimental challenge since such states obviously become more difficult to identify if they are very short lived.

In this context, recent predictions for the presence of very long-lived, high- $K$ isomers in superdeformed nuclei [105] offer an interesting possibility in the sense that the lifetimes are predicted to be so long, that even with significant mixing there may be sufficient residual $K$-hindrance that the intrinsic states are still isomeric, perhaps in the nanosecond region. The reduction in lifetime itself would constitute a highly sensitive probe of the mixing. Such mixing is also of more general interest in the elucidation of the transition from an ordered to a chaotic quantum system in hot rotating nuclei, that can, in principle, be probed through the study of isomers (see Refs. [98, 99, 100, 101] for example, and also Sect. 3.4). 


\subsection{High-K isomers in superheavy nuclei}

Compared to the lighter deformed regions, the transuranic region is relatively difficult to study. This is due to the combination of a lack of suitable beam and target combinations, together with strong fission competition in the entrance channel and in the decay of compound nuclei following fusion reactions. Nevertheless, following on the early discovery of $K$ isomers in ${ }^{250} \mathrm{Fm}$ and ${ }^{254} \mathrm{No}$ by Ghiorso et al. [282], there have been notable advances in studying long-lived states in superheavy nuclei during the past ten years (see for example Refs. [283, 284, 285, 286, 287, 288] and references therein).

There are several strong motivations for these studies. One is that the identification and characterisation of multi-quasiparticle isomers can be used to constrain the parameters that define the nuclear mean-field and thus improve the reliability of predictions in a region controlled critically by small shell effects.

Competing mean-field theories can produce very different sequences of intrinsic states near the neutron and proton Fermi surfaces. However, very limited firm experimental spectroscopic information is available on the one-quasiparticle spectrum for nuclei with $\mathrm{Z}>100$. The situation is complicated because of the difficulty in producing these nuclei under conditions suitable for spectroscopy, and also because of the combination of relatively high level densities near the ground state and the likelihood of very low-energy transitions proceeding by internal conversion. Study of high- $K$ states in the even-even nuclei offers the prospect of being somewhat more tractable since both in-band collective transitions and transitions connecting intrinsic states may well be of higher energy, while the favoured states are likely to involve the important configurations close to the Fermi surfaces, albeit as combinations of orbitals rather than in isolation. The other critical component that, in principle, can be directly extracted from experiment, is the pairing strength.

The main focus to date has been on ${ }^{254}$ No with the identification of two- and fourquasiparticle isomers $[96,289,290,291]$. While the results partly fulfill the promise outlined above, by indirectly constraining the position of the $\pi 1 / 2^{-}$[521] orbital, which arises from the spherical $2 f_{5 / 2}$ orbital above the $\mathrm{Z}=114$ shell gap, they also underline the problem of the ambiguities that arise when competing configurations give rise to states with the same quantum numbers. In the case of the $K^{\pi}=3^{+}$state, the properties (in-band branching ratios) of the associated rotational band can distinguish between alternative two-neutron or two-proton possibilities, leading to a two-proton, $\pi 1 / 2^{-}[521], 7 / 2^{-}[514]$ configuration assignment.

A similar situation arises with $K^{\pi}=8^{-}$states which can be formed by the $\pi 7 / 2^{-}[514], 9 / 2^{+}[624]$ configuration, or either of the $\nu 7 / 2^{+}[624], 9 / 2^{-}[734]$ and $\nu 7 / 2^{+}[613], 9 / 2^{-}[734]$ configurations. While in ${ }^{250} \mathrm{Fm}$ [292] and ${ }^{252} \mathrm{No}$ [293] measurements of the properties of the rotational band based on each $K^{\pi}=8^{-}$isomer have allowed a distinction to be made between the alternative configurations, with $\nu 7 / 2^{+}[624], 9 / 2^{-}[734]$ being favoured in both cases, in ${ }^{254}$ No the detailed assignments are disputed. Furthermore, definitive characterisation of the configuration of the four- 
quasiparticle, $T_{1 / 2} \approx 184 \mu$ s isomer and its decay path has been elusive, with both $K^{\pi}$ $=16^{+}$and $14^{+}$being proposed. The former case, as suggested in Refs. [96, 289], would correspond to the sum of proton and neutron $K^{\pi}=8^{-}$configurations, in analogy with the $K^{\pi}=16^{+}$isomer in ${ }^{178} \mathrm{Hf}$, but its precise excitation energy and decay properties, as well as which of the two $\nu\left[8^{-}\right]$alternatives is involved, have not been established. The suggested $K^{\pi}=14^{+}$assignment in Ref. [290] is also not firm. A cascade of somewhat shorter-lived isomers has also been reported in the heavier $N=152$ isotone ${ }^{256} \operatorname{Rf}[294,295,296]$, but the available spectroscopic information is too scarce to be able to make meaningful assignments.

While most of the information on decays from multi-quasiparticle transuranic isomers is from even-even nuclei, the $\gamma$-ray decay of three-quasiparticle isomers has been observed in odd- $N{ }^{253} \mathrm{No}[297,298,299]$ and ${ }^{257} \mathrm{Rf}[296,300,301]$, and in odd$Z{ }^{255} \operatorname{Lr}[302,303,304]$. However, the proposed decay schemes and corresponding spin/parity and configuration assignments are very tentative. Furthermore, there seem to be inconsistencies in the $E 1$ decay strengths and the decay pattern for the proposed $K^{\pi}=21 / 2^{+}$assignment of the isomer in ${ }^{257} \mathrm{Rf}$ [296].

5.4.1. Predictions for $Z=100-110$ In this context, predictions based on multiquasiparticle calculations for the nobelium and rutherfordium chain of even-even isotopes using the Woods-Saxon potential are informative [305, 306]. For example, the calculations of Kondev et al. [305] give comparable results for both chains, with three $K^{\pi}=8^{-}$alternatives lying in close proximity, as well as a single $\mathrm{K}^{\pi}=14^{+}$state, and two $\mathrm{K}^{\pi}=16^{+}$states, with different neutron-number dependencies. In fact, the configuration of the lower $K^{\pi}=16^{+}$state is expected to change between ${ }^{252}$ No and ${ }^{254}$ No. The various alternatives contribute to the difficulty in making clear associations, particularly when the experimental information is limited, as it has been to date. The significant role of $\beta_{6}$ deformation is well established $[307,308]$ and it has been emphasised recently by Liu et al. [306, 309]. Relativistic Hartree-Bogoliubov calculations aimed at investigating the role of the deformed shell gap at $N=162$ and its influence on two-quasiparticle excitation energies have recently been reported by Prassa et al. [310].

The multi-quasiparticle calculations predict that the $\mathrm{K}^{\pi}=16^{+}$state is particularly low in energy at $N=150$ in the $Z=102$ and 104 sequences, close to $2 \mathrm{MeV}$, as seen in Figure 21. Thus, ${ }^{252} \mathrm{No}$ and ${ }^{254} \mathrm{Rf}$ may have very long-lived four-quasiparticle isomers that have not been observed yet experimentally. Similar predictions have been made by Liu et al. [309], including an even more extreme case in ${ }^{270} \mathrm{Ds}$, where a lowlying $\mathrm{K}^{\pi}=20^{+}$state could be produced by a combination of favoured $K^{\pi}=10^{-}$, two-quasineutron and two-quasiproton building blocks. Candidates for the $\mathrm{K}^{\pi}=10^{-}$ components are known experimentally [311, 312, 313], but whether states as high as $K^{\pi}$ $=20^{+}$could be formed experimentally remains to be seen.

Kondev et al. [305] have suggested that the ${ }^{254} \mathrm{Rf}$ case may be of particular interest in terms of evaluating the contention that $K$-isomers could be more stable than their corresponding ground states, since identification of such a state and quantification 


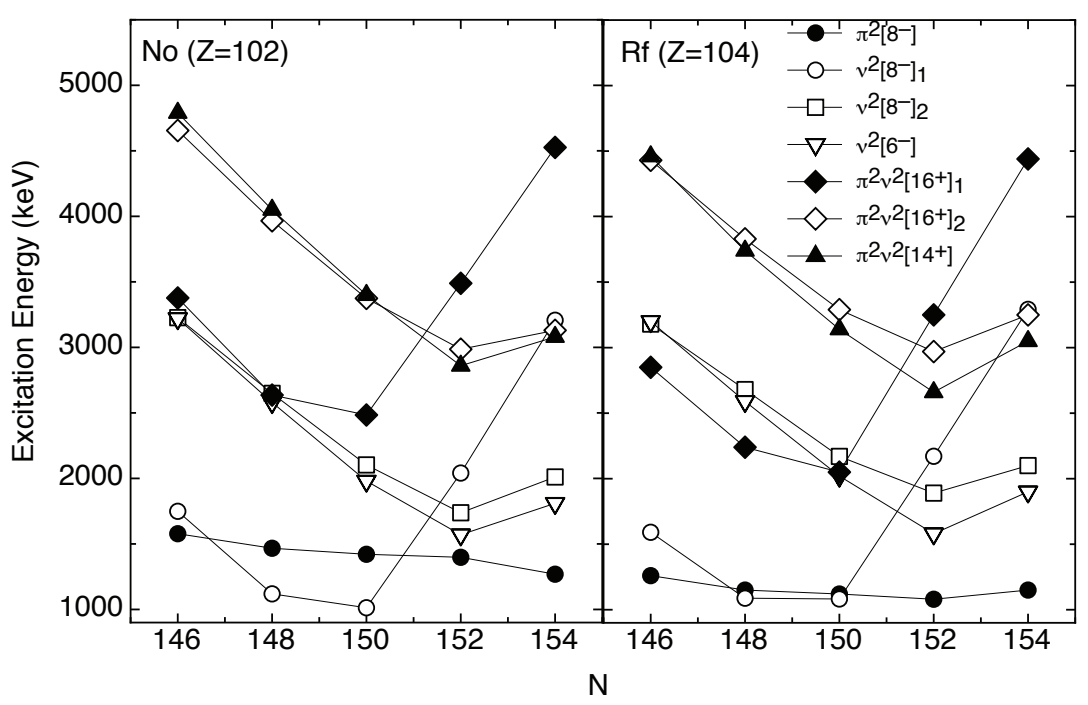

Figure 21. Predicted two- and four-quasiparticle states in No and Rf isotopes, adapted from Ref. [305].

of its decay properties would allow comparison with the ground state which decays predominantly by spontaneous fission. Indeed, a four-quasiparticle isomer has now been identified in ${ }^{254} \mathrm{Rf}$, with a half-life that is ten times longer than the ground state [314]. This issue of isomer vs. ground-state stability, especially at the limits of nuclear binding, is another motivating aspect that emerged recently [315] for pursuing studies in this region. A few cases have been found where longer-lived, excited states occur. The nuclide ${ }^{270} \mathrm{Ds}$, for example, has a two-quasiparticle, high- $K$ isomer with a half-life of $4 \mathrm{~ms}$ that is considerably longer than its $200 \mu$ s ground state [311, 312, 313]. Another case is ${ }^{250}$ No, where an isomer has a $40 \mu$ s half-life, compared to its $4 \mu$ s ground state [316]. The decay modes are $\alpha$ decay in ${ }^{270} \mathrm{Ds}$ (ground state and isomer) and fission in ${ }^{250}$ No.

In addition to early theoretical studies [317, 318, 319], new calculations have recently been carried out for the uranium, plutonium and californium isotopes [275] and specifically for the $K^{\pi}=6^{+}$and $7^{-}$isomers in ${ }^{250}$ No and ${ }^{256} \mathrm{Fm}$, respectively [320]. The contention is that specific configurations could have relatively higher and wider (onedimensional) potential barriers to fission, implying longer fission lifetimes. However, these studies do not yet include the fission dynamics (though see Refs. [321, 322]), which are likely to be crucial given the known complex dependence on shape and the fact that the process involves quantum tunnelling. Furthermore, the experimental information on fission widths of the known excited states is limited [41].

Alpha emission is one of the most common decay modes for superheavy nuclei that primarily limits their survival and (perhaps) discovery. In the presence of $\alpha$-decaying high-spin isomers, as in the case of ${ }^{270} \mathrm{Ds}$, for example, the strongest (favoured) decay is expected to proceed via an excited state in the daughter nucleus that has the same spin/parity and configuration. The direct $\alpha$ transition to the ground state would be 
severely hindered, as quantified recently by Delion et al. [323], leading to, among other issues, ambiguity in experimental assignments. It has also been shown by Rissanen et al. [324] that reduced pairing in the multi-quasiparticle states can affect the $\alpha$-decay half-life.

5.4.2. K-hindrance in superheavy nuclei Transition strengths are an important consideration in spin/parity and configuration assignments for high- $K$ states, particularly for transitions that are nominally $K$-forbidden. Unfortunately, in this region, there are only a limited number of cases where the spectroscopy is firmly established. Nevertheless, the trend, to the extent that it can be considered reliable, is that the reduced hindrances seem to be larger than in the lighter deformed regions, as reported in a recent review [41]. For example, in ${ }^{254} \mathrm{No}$, the long half-life of 266(10) ms for the $K^{\pi}=8^{-}$isomer corresponds to a very large reduced hindrance of $f_{\nu}=831$ for the 53 $\mathrm{keV}, E 1$ transition connecting it to the $I^{\pi}=7^{+}$state of the $K^{\pi}=3^{+}$band. This could be interpreted as evidence for a large configuration change, which would be consistent with either of the two-quasineutron or two-quasiproton configurations proposed for the $K^{\pi}=8^{-}$isomer, as discussed earlier, or it could imply a mis-assignment. As it stands, this strength falls at the extreme upper end of the known values for $E 1$ transitions [41].

In comparison, the highly $K$-forbidden $(\Delta K=8, \nu=7) E 1$ decays from $K^{\pi}=8^{-}$ isomers in ${ }^{246} \mathrm{Cm},{ }^{250} \mathrm{Fm}$ and ${ }^{252,254}$ No are also large $\left(f_{\nu} \approx 200\right)$ and in line with the systematic dependence as a function of the ratio of the dynamic and kinematic moments of inertia (see figure 5 of Ref. [96]). This parameter can be interpreted as a surrogate for the deformation and rotational properties, specifically those of the $I^{\pi}=8^{+}, K^{\pi}=0^{+}$ ground-state-band member to which the $E 1$ decay proceeds, as discussed in Ref. [41] and Sect. 3.4. Relatively large hindrances for $E 2$ transitions, such as the $f_{\nu}=326$ value for the ${ }^{244} \mathrm{Cm}, K^{\pi}=6^{+}$isomer decay, are also consistent with the expectation of welllocalised, axially symmetric, deformation. In comparison with similarly $K$-forbidden $E 2$ decays from two- and three-quasiparticle isomers in the $A \approx 180$ region [41, 325], only the decay of the $K^{\pi}=6^{+}$isomer in ${ }^{174} \mathrm{Yb}$ has a larger $E 2$ reduced hindrance of $f_{\nu}=340[326]$.

A different aspect of $K$ isomers in very heavy nuclei is the extent to which they might yield observables related to the octupole degree of freedom, which is already well established for some low- $K$ structures [142]. In particular, it has been suggested that the magnetic dipole moments of isomers can be sensitive to static octupole deformation [258], and potential-energy-surface calculations [259] indicate that the $K^{\pi}=6^{-}$isomer of ${ }^{234} \mathrm{U}$ is especially promising in this regard.

\section{Isomer targets}

Where isomeric states are sufficiently long lived (years or greater) it is possible to use them for reaction studies, though target purity is likely to be a limiting factor. Even with the "stable" isomer of ${ }^{180} \mathrm{Ta}\left(T_{1 / 2}>10^{16} \mathrm{yr}[327]\right)$ the natural abundance of $0.01 \%$ 
compared to ${ }^{181} \mathrm{Ta}$ limits enrichment to the few percent level. Nevertheless, key photoexcitation and Coulomb excitation measurements have been possible [29, 30, 328] (see also Sect. 8). Another accessible target is the $141 \mathrm{yr}$ isomer of ${ }^{242} \mathrm{Am}$, where remarkable purity (98\%) was obtained for Coulomb excitation studies [329].

Although approaching the limits for target production, the $31 \mathrm{yr}$ isomer of ${ }^{178} \mathrm{Hf}$ has received particular attention on account of its high spin $(16 \hbar)$ and excitation energy $(2.4 \mathrm{MeV})$. In addition to photo-activation and photo-destruction (see Sect. 8) it has been possible to study inelastic deuteron scattering [330], two-neutron transfer [331] and neutron capture [332] reactions. Furthermore, inelastic neutron acceleration has been identified with a ${ }^{178} \mathrm{Hf}$ isomer target [333], as well as from a ${ }^{177} \mathrm{Lu}$ isomer target $\left(T_{1 / 2}=160\right.$ days) $[334,335]$, building on earlier reports, e.g. with a ${ }^{180} \mathrm{Hf}$ isomer target $\left(T_{1 / 2}=5.5\right.$ hours $)[336]$.

Despite these successes, the half-life limitation in the production of isomer targets severely limits the range of experimental possibilities. However, the availability of radioactive beams now opens up a range of new opportunities, as discussed in the following sections.

\section{Isomer beams}

Rykaczewski et al. [337] pointed out early possibilities with isomeric beams produced in fragmentation, and recently Walker [338] has also discussed the use of isomeric beams for nuclear structure studies and other applications, including the issues of beam purification and decay rates (see also Sect. 10.4). In another approach, Ishii et al. [339] have demonstrated the development of beams of isomeric projectile-like fragments from deepinelastic reactions, for studing the products themselves. Other interesting prospects reside with producing high-spin isomers in relatively heavy nuclei (either with fusionevaporation or deep-inelastic reactions) and using these as samples for (self) excitation or even as beams to instigate secondary reactions. Coulomb excitation of a $K$-isomer in ${ }^{174} \mathrm{Hf}\left(T_{1 / 2}=2.4 \mu \mathrm{s}\right)$ produced as a secondary beam was demonstrated by Morikawa et al. [340], giving access to matrix elements and structures that cannot be reached directly from the ground states of such nuclei. Gono et al. have pursued a number of approaches [341, 342] producing beams of $J^{\pi}=27^{+},{ }^{144} \mathrm{Pm}\left(T_{1 / 2}=2.7 \mu \mathrm{s}\right)$ and $49 / 2^{+}$, ${ }^{145} \mathrm{Sm}\left(T_{1 / 2}=1 \mu \mathrm{s}\right)$ for example, while in Ref. [343] the reaction mechanism was studied using ${ }^{145 m} \mathrm{Sm}$ as a beam to instigate $\left({ }^{12} \mathrm{C}, x \mathrm{n}\right)$ reactions, thus reaching an entry region not normally accessible with conventional reactions.

Direct studies of fusion and the consequences of using exotic isomeric beams represent a likely productive direction for the future. The sensitivity of fusion between heavy nuclei at energies near the Coulomb barrier to the structure of both target and projectile [344], and the fact that very low cross sections can be measured if the product nuclei are detected, rather than by using indirect studies with $\gamma$-rays, for example, make this approach attractive. Significant cross-section differences will arise using a projectile (or target) of something like the ${ }^{178} \mathrm{Hf}$ isomer, whose large angular momentum of 
$K^{\pi}=16^{+}$would dramatically skew the input $l$-distributions compared to normal beams. Furthermore, Butt et al. [345] have demonstrated that fission angular distributions following fusion are very sensitive to the input angular momentum, providing another probe of the reaction mechanism. These predictions could be tested equally well with other isomers such as the $K=15$ state in ${ }^{184} \mathrm{Hf}$ identified in storage-ring measurements $[82,83]$. See also Sect. 8.4.

\section{Energy traps, induced deexcitation, lasers and astrophysics}

Every radionuclide stores energy, and the gradual release of that energy can, in principle, be used to power external devices, as is the case for the thermoelectric supplies common in space-craft that exploit the "heat" from the $\alpha$ decay of ${ }^{238} \mathrm{Pu}$. However, in general, the natural half-life will limit the applications since the energy is released at a pre-defined and probably unsuitable rate. Isomers may offer a path to future applications if this rate can be modified and/or controlled. Indeed, the potential to use relatively low-energy electromagnetic interactions, ideally photons from a laser or electrons in a plasma, to stimulate the release of the isomer's energy has attracted considerable attention (see for example Refs. [17, 19, 20, 21, 22, 23, 346, 347, 348, 349]). For illustration, a partial level scheme for ${ }^{129} \mathrm{Sb}$ is shown in figure 22 (see also Sect. 8.4).

Various schemes are envisaged. In the case of the $T_{1 / 2}=31 \mathrm{yr}, 2.4 \mathrm{MeV}$ isomer of ${ }^{178} \mathrm{Hf}$, for example, spontaneous decay directly to the ground state does not occur, but if a transition from the isomer to a higher-lying, "intermediate" or "gateway" state could be stimulated, then decay could proceed to the ground state, bypassing the isomer and releasing the stored energy. One problem for the ${ }^{178} \mathrm{Hf}$ isomer is that a suitable intermediate state has not been identified, so the required stimulation energy is unknown. This contrasts with ${ }^{129} \mathrm{Sb}$ (figure 22) where a $10 \mathrm{keV}, E 2$ excitation from the isomer would be appropriate. However, the short half-life (18 min.) of the ${ }^{129} \mathrm{Sb}$ isomer means that macroscopic quantities cannot be produced for experimental study.

8.1. ${ }^{180} \mathrm{Ta}$

Attempts were made to demonstrate energy release in the laboratory from the $75 \mathrm{keV}$, $K^{\pi}=9^{-}$isomer in ${ }^{180} \mathrm{Ta}$, whose natural half-life is more than $10^{16} \mathrm{yr}$ [327], in contrast to the short half-life ( 8.2 hours) of the ground state. Although early results were controversial, definitive photo-destruction of this isomer has been achieved with photon energies as low as $1 \mathrm{MeV}[29,30]$. While that energy is much greater than the $75 \mathrm{keV}$ of the isomer itself, so there is no real gain, this at least establishes the principle of isomer photo-destruction with energies well below the neutron binding energy.

Belic et al. [29, 30] in fact identified a series of strong resonances in the photoactivation yield, associated presumably with individual (or perhaps groups) of specific states in ${ }^{180} \mathrm{Ta}$. Candidates for such states have been proposed [31] but searches for the $\gamma$-ray branches, predicted on the basis of an analysis of the large resonance cross 


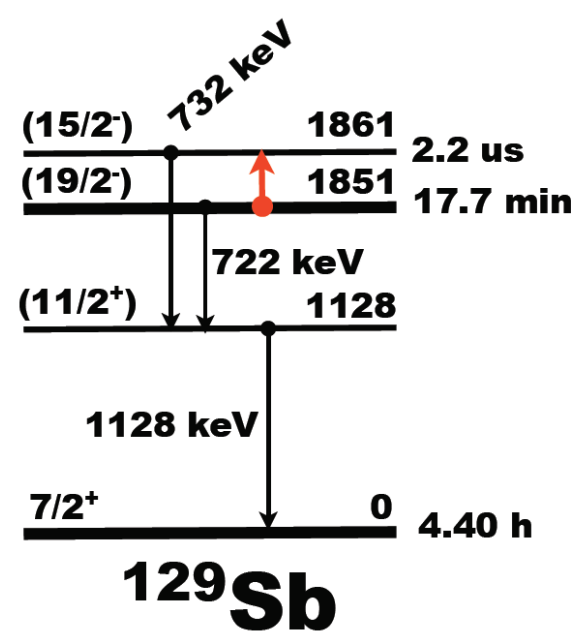

Figure 22. Partial level scheme for ${ }^{129} \mathrm{Sb}$, illustrating the $18 \mathrm{~min}$. isomer and a possible $E 2,10 \mathrm{keV}$ transition (up-pointing arrow, in red) that would enable its deexcitation via the $732 \mathrm{keV}$ transition from the $1861 \mathrm{keV}, 2.2 \mu$ s isomer.

sections, have yet to be identified [32]. This interpretation [31] also suggests that the E1 strengths involved are much larger than might be expected on the basis of the normal rates and the nominal $K$-changes. (For example, Ref. [350] postulates that octupole effects might need to be invoked.) The existence and character of states that could explain the large yields thus remains a significant issue for the understanding of both the process and the underlying nuclear structure. The "sister" nuclide ${ }^{176} \mathrm{Lu}$ will be discussed below in the context of stellar environments.

\section{2. ${ }^{178} \mathrm{Hf}$}

Given the imperative (in applications) of producing a significant energy gain, it is not surprising that the the $2.447 \mathrm{MeV}, T_{1 / 2}=31 \mathrm{yr}, K^{\pi}=16^{+}$isomer of ${ }^{178} \mathrm{Hf}$ has been intensively studied. As well as the high excitation energy implying high energy storage, an attraction of highly excited isomeric states is, at least superficially, because the high energy increases the likelihood of other short-lived states with similar quantum numbers falling in the immediate vicinity. States that could be connected by $E 1$ photons in the $\mathrm{eV}$ region might provide a path for excitation using lasers, while a high density of states of the same spin and parity (but different $K$ ) would lead to statistical $K$-mixing which could again provide an accessible pathway for deexcitation. However, the reason for the long lifetime of the ${ }^{178} \mathrm{Hf}$ isomer is because it is very yrast, that is, it lies well below other states of the similar spin, nullifying this argument.

Although positive indications for the destruction of the ${ }^{178} \mathrm{Hf}$ isomer using $<100$ $\mathrm{keV}$ photons were reported over a decade ago [351] and continued (see for example Ref. [352]), other measurements have established very low cross-section limits [353, 354] 
which effectively refute the earlier claims.

In parallel with such direct measurements, nuclear structure calculations have suggested that $\gamma$ deformation could play an important role in the photo-destruction of the ${ }^{178} \mathrm{Hf}$ isomer because it would result in $K$-mixing near the yrast line [355]. Furthermore, Coulomb excitation studies of ${ }^{178} \mathrm{Hf}$ leading indirectly to the isomer from the $K^{\pi}=0^{+}$ground state have identified significant (and unexpected) mixing in a variety of low- $K$ bands as well as evidence for an aligned near-yrast band providing possible paths for the (nominally forbidden) excitation of high- $K$ states [356, 357, 358].

While these are positive, if somewhat imprecise, aspects, a factor weighing against the possibility of successful low-energy photo-destruction of the ${ }^{178} \mathrm{Hf}$ isomer is the lack, to date, of a suitable higher-lying intermediate state. Indeed, this may be a general problem for high- $K$ isomers in deformed nuclei. If large changes in the $K$ quantum number are involved, it can be argued [179] that intermediate- $K$ states are unlikely to have the necessary properties since the stimulating transition needs to have a large matrix element. A significant change in $K$ implies either a high-multipole allowed transition or a low-multipole $K$-forbidden transition and also a substantial change in intrinsic structure, all of which would imply slow transitions. In a specific case, Karpeshin et al. [359] consider resonance conversion and the possibility of exciting via an $M 2$ transition to the $K^{\pi}=14^{-}, 68 \mu$ s isomer, $126 \mathrm{keV}$ above $K^{\pi}=16^{+}$isomer.

\subsection{NEEC and NEET}

In the consideration of low-energy mechanisms for isomer de-population, the coupling to atomic electrons seems especially promising. The principal processes are nuclear excitation by electronic transition (NEET), which was first identified in ${ }^{197} \mathrm{Au}$ as an excitation from the nuclear ground state [360], and nuclear excitation by free electron capture (NEEC), which is the inverse of electron conversion, but is a process yet to be observed. Both NEEC and NEET require appropriately matched atomic-shell vacancies, which can occur in plasmas [361] and energetic ion beams [22].

Several papers tabulate isomers that are potentially suitable for experimental investigation with plasmas $[22,362,363]$, where the basic requirement is a small energy difference between the isomer and the intermediate state. A good candidate for further study is the $2.4 \mathrm{MeV}, T_{1 / 2}=7 \mathrm{hr}, J^{\pi}=21 / 2^{+}$isomer of the spherical nuclide ${ }^{93} \mathrm{Mo}$ $[22,361]$ that has a structurally related $J^{\pi}=17 / 2^{+}$state only $5 \mathrm{keV}$ above the isomer. [364]. According to the usual seniority-isomer structure, the $17 / 2^{+}$state would have slightly lower energy than the $21 / 2^{+}$state, with the possibility of $E 2$ decay from the $21 / 2^{+}$state. However, residual proton-neutron interactions lead to the $17 / 2^{+}$state being at higher energy. The $21 / 2^{+}$state is then forced to decay by $E 4$ radiation to the $13 / 2^{+}$ state, resulting in the long lifetime. Furthermore, induced excitation to the $17 / 2^{+}$state is calculated to have a large matrix element of 3.5 W.u. [364]. Similar situations exist in other near-spherical nuclei, including the ${ }^{212} \mathrm{Bi}\left(18^{-}\right)[179]$ and ${ }^{212} \mathrm{Po}\left(18^{+}\right)[165,172]$ isomers, although in each of these cases the energy of a specific (calculated) intermediate 
state is not known experimentally.

Recently, a detailed theoretical evaluation has been given of the prospects for secondary (resonant) nuclear photoexcitation exploiting intense X-ray free-electron lasers [365], for the specific case of ${ }^{93}$ Mo mentioned above. The claim is made that secondary processes in the plasma result in an increase by orders of magnitude over direct laser-nucleus excitation.

\subsection{Radioactive beams}

From the point of view of the experimental investigation of isomer photo-depopulation, the ${ }^{180} \mathrm{Ta}$ and ${ }^{178} \mathrm{Hf}$ isomers are favourable due to their long half-lives and the consequent ability to make isomer targets, albeit with low purity. However, with the advent of intense radioisotope beams, the half-life constraint is now much less severe, at least for the investigation of the physical mechanisms of photo-depopulation, although probably not with respect to the eventual aim for an energy-storage device. While not applied to a heavy nucleus, a milestone in this regard has been the ISOL production, laser separation, re-acceleration and Coulomb depopulation of the $J^{\pi}=6^{-}, T_{1 / 2}=4 \mathrm{~min}$ isomer of ${ }^{68} \mathrm{Cu}[366]$. See also Sect. 10.4.

Isomers that are potentially suitable for further study have been detailed by other authors $[22,346,362,363]$, but only for half-lives greater than $20 \mathrm{~min}$. In table 5 we list isomers with half-lives in the range $20 \mathrm{~s}$ to $20 \mathrm{~min}$., with the additional requirement that the excitation energy should be greater than $1 \mathrm{MeV}$. The latter restriction means that significant energy release can be expected while the minimum half-life of $20 \mathrm{~s}$ is appropriate for storage-ring measurements.

The heavy nuclei $(A>180)$ in the table do not have known intermediate states that are good candidates for photo-excitation. However, the spherical nuclei $(A>200)$ are expected to have such states, since the long half-lives are due to the absence of available $E 2$ decays, with the corresponding states having been pushed above the isomer energy by residual interactions. These are therefore attractive for future study. An additional experimental consideration is the need to distinguish the normal (spontaneous) decay $\gamma$ rays from induced deexcitation $\gamma$ rays. While at least one of the induced $\gamma$ decays would anyway have a unique energy (i.e. the decay from the intermediate state) there could be an additional advantage if the isomer itself has little or no IT decay. This is true for several cases, notably ${ }^{52} \mathrm{Fe}$ and ${ }^{130} \mathrm{Sn}$, where the isomers $\beta$ decay, and ${ }^{211,212} \mathrm{Po}$, where the isomers predominantly $\alpha$ decay.

A novel measurement envisaged exploits the planned installation of the low-energy $C R Y R I N G$ at GSI, fed by beams from the Experimental Storage Ring (ESR) [368]. At this facility it will be possible to slow down bare isomeric ions, such as those of the species just mentioned, to energies well below the Coulomb barrier. If these ions were then implanted into a solid, the immediate capture of a large number of electrons would give the possibility of exciting the isomer by NEEC and NEET processes, releasing the isomer energy through a cascade of $\gamma$ rays. At least one of the $\gamma$ rays would be unique to 
Table 5. Isomers with $20 \mathrm{~s}<T_{1 / 2}<20 \mathrm{~min}$ and $E^{*}>1 \mathrm{MeV}$. Data are from Ref. [44] unless otherwise indicated. In addition to the half-life, spin-parity assignment (mostly tentative) and isomer excitation energy, the additional energy needed to reach a possible intermediate state is given, together with the multipole character of that transition, and the isomer decay mode ( $\alpha$ decay, $\beta$ decay or internal transition).

\begin{tabular}{cccccccc}
\hline Nuclide & $T_{1 / 2}$ & $I^{\pi}$ & $E^{*}(\mathrm{keV})$ & $\Delta E(\mathrm{keV})$ & $\sigma \lambda^{\text {a) }}$ & decay mode & Ref. \\
\hline${ }^{52} \mathrm{Fe}$ & $46 \mathrm{~s}$ & $12^{+}$ & 6958 & 424 & $E 2$ & $\beta$ & \\
${ }^{53} \mathrm{Fe}$ & $2.5 \mathrm{~m}$ & $19 / 2^{-}$ & 3040 & 422 & $E 2$ & $I T$ & \\
${ }^{129} \mathrm{Sb}$ & $18 \mathrm{~m}$ & $19 / 2^{-}$ & 1851 & 10 & $E 2$ & $I T$ & \\
${ }^{130} \mathrm{Sn}$ & $1.7 \mathrm{~m}$ & $7^{-}$ & 1947 & 138 & $E 2$ & $\beta$ & \\
${ }^{184} \mathrm{Hf}$ & $48 \mathrm{~s}$ & $8^{-}$ & 1272 & - & - & $\beta+I T$ & {$[83]$} \\
${ }^{184} \mathrm{Hf}$ & $12 \mathrm{~m} \mathrm{b)}$ & $15^{+}$ & 2477 & - & - & $\beta$ & {$[83]$} \\
${ }^{186} \mathrm{Hf}$ & $>20 \mathrm{~s})$ & $17^{+}$ & 2968 & - & - & & {$[83]$} \\
${ }^{187} \mathrm{Ta}$ & $22 \mathrm{~s})$ & $15^{+}$ & 1793 & - & - & $\beta+I T$ & {$[83]$} \\
${ }^{187} \mathrm{Ta}$ & $>5 \mathrm{~m} \mathrm{~b})$ & $41 / 2^{+}$ & 2933 & - & - & $\beta+I T$ & {$[83]$} \\
${ }^{190} \mathrm{Os}$ & $10 \mathrm{~m}$ & $10^{-}$ & 1705 & - & - & $I T$ & \\
${ }^{206} \mathrm{Tl}$ & $3.7 \mathrm{~m}$ & $12^{-}$ & 2643 & - & $(E 2)$ & $I T$ & \\
${ }^{211} \mathrm{Po}$ & $25 \mathrm{~s}$ & $25 / 2^{+}$ & 1462 & - & $(E 2)$ & $\alpha$ & \\
${ }^{212} \mathrm{Po}$ & $45 \mathrm{~s}$ & $18^{+}$ & 2923 & - & $(E 2)$ & $\alpha$ & \\
${ }^{212} \mathrm{Bi}$ & $7 \mathrm{~m}$ & $18^{-}$ & 1478 & - & $(E 2)$ & $\beta+I T$ & {$[179]$} \\
${ }^{213} \mathrm{Bi}$ & $>168 \mathrm{~s})$ & $25 / 2^{-}$ & 1353 & - & $(E 2)$ & $I T$ & {$[367]$} \\
${ }^{215} \mathrm{Bi}$ & $37 \mathrm{~s}$ & $25 / 2^{-}$ & 1348 & - & $(E 2)$ & $\beta+I T$ & \\
\hline
\end{tabular}

a Parentheses indicate that a low-energy $E 2$ transition is expected from shell-model considerations.

b The quoted half-life is for highly charged ions in a storage ring.

the induced depopulation pathway, thus providing a signature for the nuclear excitation together with information about depopulation probabilities. One such candidate [368] is the $T_{1 / 2}=18 \mathrm{~min}$., $1851 \mathrm{keV}$ isomer of ${ }^{129} \mathrm{Sb}$, whose level structure is illustrated in figure 22 .

\subsection{Isomers and nucleosynthesis}

A related issue is the effective lifetime and production/survival of isomers in astrophysical environments, where there is Maxwellian population of excited states and where the photon flux can both excite and de-excite isomers [26, 27, 369]. The photo-destruction of ${ }^{180 \mathrm{~m}} \mathrm{Ta}$, discussed above, has been of particular interest given the challenge of explaining its extremely low but finite natural abundance on Earth [29, 30, 31, 34, 370, 371, 372, 373].

Its sister odd-odd isotope ${ }^{176} \mathrm{Lu}$, produced in the slow neutron-capture process $(s$ process) exhibits a complementary structure. It has a very long-lived $K^{\pi}=7^{-}$ground state, and a relatively short-lived $K^{\pi}=0^{-}, J^{\pi}=1^{-}, \beta$-decaying isomer at $123 \mathrm{keV}$. The states are formed by the parallel and anti-parallel coupling of the same pair of proton and neutron orbitals. The $\beta$-decaying isomer will be more strongly populated than the ground state by neutron capture in stellar environments [374] as shown in the laboratory 
through the use of a quasi-Maxwellian neutron beam that mimics a temperature of $\mathrm{kT}=25 \mathrm{keV}$. Furthermore, photo-excitation via higher-lying intermediate- $K$ states provides a dual pathway $\left(7^{-} \rightarrow 0^{-}\right.$or $\left.0^{-} \rightarrow 7^{-}\right)$which can depopulate or populate the long-lived ground state. Together with the initial neutron-capture rates, these factors affect the production rates of ${ }^{176} \mathrm{Lu}$ and its hafnium daughter [33, 371, 375, 35]. Evidence from nuclear spectroscopy for the existence of intermediate states which could play a role in this process is still fragmentary and a consensus has not been reached (see for example Ref. [33]). Laboratory experiments on photo-destruction of the quality of those available for ${ }^{180} \mathrm{Ta}$ have yet to be reported.

Another interesting example in the same mass region is the very long-lived $\left(\mathrm{T}_{1 / 2}=2.0 \times 10^{5}\right.$ years $) \mathrm{K}^{\pi}=8^{+}$isomer in the odd-odd ${ }^{186} \mathrm{Re}$ nuclide, which can influence the ${ }^{187}$ Re- ${ }^{187}$ Os rapid neutron-capture process ( $r$-process) cosmo-chronometer proposed by Clayton [376]. Still little is known about the structure of the ${ }^{186}$ Re isomer and the excited states above it, which are needed to improve predictions of the various production and destruction cross sections of the isomer. While recent activation studies [377] indicated that the isomer may be of lesser importance to the ${ }^{187} \mathrm{Re}^{187}$ Os clock, the results depend sensitively on the half-life of the isomer, which is not yet well established.

For neutron-rich nuclides produced in $r$-process nucleosynthesis, detailed properties of $\beta^{-}$- and $\beta_{n}^{-}$-decaying isomers are needed for various network calculations $[369,378$, 379]. It is worth noting that important information relevant to the $r$-process has already been obtained from isomer spectroscopy, see for example Refs. [380, 381, 382]. With the development of the new rare-isotope beam facilities and improved experimental techniques, the discovery of neutron-decaying isomers [90] could also be within reach in the foreseeable future.

\section{Isomers for medicine}

Radioisotopes are widely used in medicine for imaging and therapeutic applications [25, 24, 383]. Isomers are particularly suitable for Single Photon Emission Computer Tomography (SPECT) imaging, where a single and relatively low-energy $\gamma$ ray is emitted. The most used SPECT isomer is ${ }^{99 m} \mathrm{Tc}\left(T_{1 / 2}=6\right.$ hours) which decays via a $142 \mathrm{keV} \gamma$ ray that can be easily detected with high efficiency. Other isomers that are used in medical applications are shown in table 6. Longer-lived isomers, such as ${ }^{193 m} \mathrm{Pt}$ and ${ }^{195 m} \mathrm{Pt}$, for example, are used in cancer treatments. Because of the high multipole order of the depopulating transitions, most of the decays occur via internal conversion, with subsequent cascade emission of Auger electrons that can be used to kill various cancer cells. Many of the known isomers have relatively short half-lives, which preclude their direct use in medical applications. However, there are cases where decays (mostly $\beta^{-}, \beta^{+}$or EC) of a longer-lived parent nuclide can populate the isomer via the so-called generator scheme. A number of such generator pairs that involve isomer decays are included in table 6 . There are also a few cases where the isomer decays by positron emission and is suitable for Positron Emission Tomography (PET) imaging, with the 
Table 6. Isomers commonly used in medical applications. Data are from Ref. [44].

\begin{tabular}{|c|c|c|c|c|}
\hline Generator/Nuclide & $T_{1 / 2}$ & $E^{*}(\mathrm{keV})$ & decay mode & Application \\
\hline${ }^{34 m} \mathrm{Cl}$ & $31.99 \mathrm{~m}$ & 146.360 & $\beta^{+}$ & PET \\
\hline${ }^{52} \mathrm{Fe} /{ }^{52 m} \mathrm{Mn}$ & $8.275 \mathrm{~h} / 21.1 \mathrm{~m}$ & 377.749 & $\mathrm{EC} / \beta^{+}$ & PET \\
\hline${ }^{80 m} \mathrm{Br}$ & $4.4205 \mathrm{~h}$ & 85.843 & IT & Auger \\
\hline${ }^{81} \mathrm{Rb} /{ }^{81 m} \mathrm{Kr}$ & $4.572 \mathrm{~h} / 13.10 \mathrm{~s}$ & 190.64 & $\mathrm{EC} / \beta^{+} / \mathrm{IT}$ & SPECT;PET \\
\hline${ }^{82 m} \mathrm{Rb}$ & $6.472 \mathrm{~h}$ & 69.0 & $\beta^{+}$ & PET \\
\hline${ }^{94 m} \mathrm{Tc}$ & $52.0 \mathrm{~m}$ & 76.0 & $\beta^{+}$ & PET \\
\hline${ }^{99} \mathrm{Mo} /{ }^{99 m} \mathrm{Tc}$ & $65.976 \mathrm{~h} / 6.0067 \mathrm{~h}$ & 142.6832 & $\beta^{-} / \mathrm{IT}$ & SPECT \\
\hline${ }^{103} \mathrm{Pd} /{ }^{103 m} \mathrm{Rh}$ & $16.991 \mathrm{~d} / 56.114 \mathrm{~m}$ & 39.753 & $\mathrm{EC} / \mathrm{IT}$ & Auger \\
\hline${ }^{113} \mathrm{Sn} /{ }^{113 m} \mathrm{In}$ & $115.09 \mathrm{~d} / 1.6579 \mathrm{~h}$ & 391.699 & $\mathrm{EC} / \mathrm{IT}$ & SPECT; Auger \\
\hline${ }^{115 m} \mathrm{In}$ & $4.486 \mathrm{~h}$ & 336.244 & IT & SPECT \\
\hline${ }^{117 m} \mathrm{Sn}$ & $14.0 \mathrm{~d}$ & 314.58 & IT & SPECT;Auger \\
\hline${ }^{167} \mathrm{Tm} /{ }^{167 m} \mathrm{Er}$ & $9.25 \mathrm{~d} / 2.269 \mathrm{~s}$ & 207.801 & $\mathrm{EC} / \mathrm{IT}$ & SPECT;Auger \\
\hline${ }^{178} \mathrm{Ta} /{ }^{178 m} \mathrm{Hf}$ & $2.36 \mathrm{~h} / 4.0 \mathrm{~s}$ & 1147.416 & $\mathrm{EC} / \mathrm{IT}$ & SPECT; Auger \\
\hline${ }^{193 m} \mathrm{Pt}$ & $4.33 \mathrm{~d}$ & 149.78 & IT & Auger \\
\hline${ }^{195 m} \mathrm{Pt}$ & $4.01 \mathrm{~d}$ & 259.30 & IT & Auger \\
\hline${ }^{191} \mathrm{Os} /{ }^{191 m} \mathrm{Ir}$ & $14.99 \mathrm{~d} / 4.899 \mathrm{~s}$ & 171.29 & IT & SPECT;Auger \\
\hline
\end{tabular}

most notable cases being ${ }^{34 m} \mathrm{Cl}$ and ${ }^{52 m} \mathrm{Mn}$.

\section{Key experimental developments}

For nuclear structure investigations, the continuing challenge is to produce and characterise exotic nuclei that, in general, are not accessible with conventional reactions. Selected aspects of this challenge are discussed in the following sections.

\subsection{Neutron-deficient and very heavy nuclei}

10.1.1. Recoil-decay tagging and related techniques As stated earlier, identification of the transitions feeding isomers is important for their characterization, but for some of the new techniques this is often impractical because of the large fluxes of prompt radiation produced at the targets, particularly in high-energy collisions.

An important historical development was that of the "recoil shadowing" technique where the forward momentum of reaction products enables them to fly out of the target, and detectors of isomeric radiations can then be shielded from the direct emissions from the target position. This was instrumental in the discovery of many fission $[269,270]$ and high-spin, $\gamma$-ray decaying isomers [36, 384], including the nine-quasiparticle $K$-isomer in ${ }^{175} \mathrm{Hf}[84]$.

For heavy-ion induced reactions, the high forward momentum enables in-flight selection techniques to be applied $[385,386]$ and in some cases $A$ and $Z$ identifications can be achieved on an ion-by-ion basis. The above approach made use of recoilseparation devices with several metres flight part and flight times that are significant fractions of a microsecond (at least). Thus, half-lives of hundreds of nanoseconds and 
longer are typically required for the isomeric states, in order for these to be detected. The recoil separators themselves (for fusion-evaporation reaction products) fall into two broad categories: vacuum separators and gas-filled separators [385, 387]. The former can give the best mass resolution for individual charge states, while charge averaging in the latter leads to a poorer mass resolution, but greater efficiency. After passing through the separator, the reaction residues are typically implanted into highlysegmented (pixelated) silicon detectors, located in the focal plane of the separator, and are correlated in space (within a single pixel) and time with the subsequent proton, conversion electron, $\alpha$-particle and/or fission decays. This allows decay properties of the stopped recoils to be studied in a reduced-background environment and to achieve an unprecedented sensitivity. Gamma rays emitted in the decay of isomers can also be detected at the focal plane using high-resolution germanium detectors. By the same token, the observation of prompt radiations from the target can be correlated in time with physically separated decay radiations, and this is referred to as recoildecay tagging (RDT) [388, 389]. The upper limit on the prompt-delayed correlation times depends on the counting rates, and for delayed proton and $\alpha$-particle decays, for example, correlations over many seconds have been observed.

The RDT technique is the essential tool for studying properties of isomers in neutron-deficient nuclei, where proton and $\alpha$-particle emissions are the main decay modes. A wealth of such studies were carried out in the last decade by coupling mass separators with the large $\gamma$-ray arrays, such as GAMMASPHERE at ANL (USA) and JUROSPHERE and JUROGAM at the University of Jyväskylä (Finland). For proton-emitting nuclei at the drip line, RDT studies were carried out mainly for lowseniority isomers [386], with a notable example being the observation of rotational bands above the $\mathrm{I}^{\pi}=7 / 2^{-}$ground state and the $\mathrm{I}^{\pi}=1 / 2^{+}$isomer in ${ }^{141} \mathrm{Ho}$ [390]. Many RDT studies for neutron-deficient nuclei in the $Z=70-88$ region were performed using $\alpha$-decay tagging. For example, the observed structures above the $\pi h_{11 / 2}$ isomers in odd- $Z$ Re, Ir and Au isotopes [391, 392, 393, 394] and the $\nu i_{13 / 2}$ isomers in odd- $N$ Pt and $\mathrm{Hg}$ nuclei [395, 396, 397, 398, 399, 400] allowed not only characterization of the structure of the isomers, but also revealed a unique interplay between competing shapecoexisting structures. Prompt radiations were also established above the $\alpha$-decaying, multi-quasiparticle isomers in ${ }^{151} \mathrm{Lu}$ [401], ${ }^{155} \mathrm{Lu}$ [402], ${ }^{156} \mathrm{Hf}[403]$ and ${ }^{158} \mathrm{Ta}$ [403, 404], whose structures are similar to those known in the $Z \sim 64$ and $N \sim 82$ region, as discussed in Sect. 4.2.

Initially, fission tagging was applied in studies of superdeformed isomers in the second well, where a key milestone was the observation of conversion electrons from rotational states above the $4 \mathrm{~ns},{ }^{240} \mathrm{Pu}$ fission isomer [271, 405]. Recent studies by means of $\alpha$-decay and fission tagging were instrumental in discovering excited structures in very heavy nuclei $[285,286,288]$. Of particular importance to isomer research was the technique proposed by Jones [406], where correlations are established between the $\alpha$-particle or fission decaying ground state and conversion electrons (CE) produced in the decay path of a long-lived isomer. This approach allowed the discovery of many 
isomers in very heavy nuclei in the trans-fermium region [285, 286, 288]. When there is a cascade of isomers, CE tagging at the focal plane of the mass separator can also be implemented, as demonstrated in the discovery of the four-quasiparticle isomer in ${ }^{254} \mathrm{No}[96,289,290,291]$ and the suite of isomers in ${ }^{256} \mathrm{Rf}[294,295]$. In addition, using the conventional RDT method, CE can be correlated with the in-beam $\gamma$ rays emitted at the target position. Rotational band structures above the $K^{\pi}=8^{-}$isomers in ${ }^{250} \mathrm{Fm}[292]$ and ${ }^{252}$ No [293] were identified using this approach, which provided valuable information about the possible configurations.

Of special interest for isomer studies is the correlation of prompt $\gamma$ rays from the target with delayed $\gamma$ rays from the stopper, first used by Cullen et al. [407] to identify rotational states above the $6 \mu \mathrm{s}, K^{\pi}=8^{-}$isomer of ${ }^{138} \mathrm{Gd}$. Since the recoil-ion properties do not in themselves enable an isomer to be distinguished from its corresponding ground state (unless injected into a storage ring - see Sect. 10.2.5) the ability to correlate with uniquely specific decay energies is of particular value. The application of the technique to delayed $\beta$ decays has proved more problematical, due to the continuous nature of the $\beta$-decay energy spectrum, though there has been some success $[408,409]$ including the first identification of excited states in ${ }^{78} \mathrm{Y}$.

For shorter half-lives, it may be preferable to have a target that is sufficiently thick to stop the reaction products, and use pulsed-beam techniques to help select the isomeric radiations.

\subsection{Neutron-rich nuclei}

10.2.1. Relativistic projectile fragmentation Fragmentation of heavy beams at relativistic energies (approaching $1 \mathrm{GeV} /$ nucleon) combined with advanced magnetic separators has been shown to be very effective in the production and identification of neutron-rich isotopes of heavy nuclei and the identification of at least medium-spin isomers in many of them [337]. The reaction properties have been quantified in terms of the key factors that govern their utility, such as the cross sections as a function of separation from the incident beam $[192,410,411]$ and angular-momentum input/highspin population $[410,412]$. The very high velocities mean that fully stripped ions of even the heaviest nuclei predominate, and thus unambiguous $Z$ and $A$ identification [192] is possible.

These techniques have been used successfully to identify states in a range of relatively heavy nuclei, including, for example, the seniority isomers in the neutron-rich lead isotopes discussed earlier [193] extending the original study of Pfützner et al. [192], high-spin states and isomers in the tungsten-osmium region [413, 414], and discoveries in many other nuclei in the mass 190-200 region (see for example Refs. [415, 416, 417, 418]). The early studies were somewhat limited in terms of the extent of firm spectroscopic information, although the situation continues to develop with the incorporation of more powerful $\gamma$-ray detections systems such as used in the RISING campaign [419]. Figure 23 is an example of the reach of these reactions for populating neutron-rich 


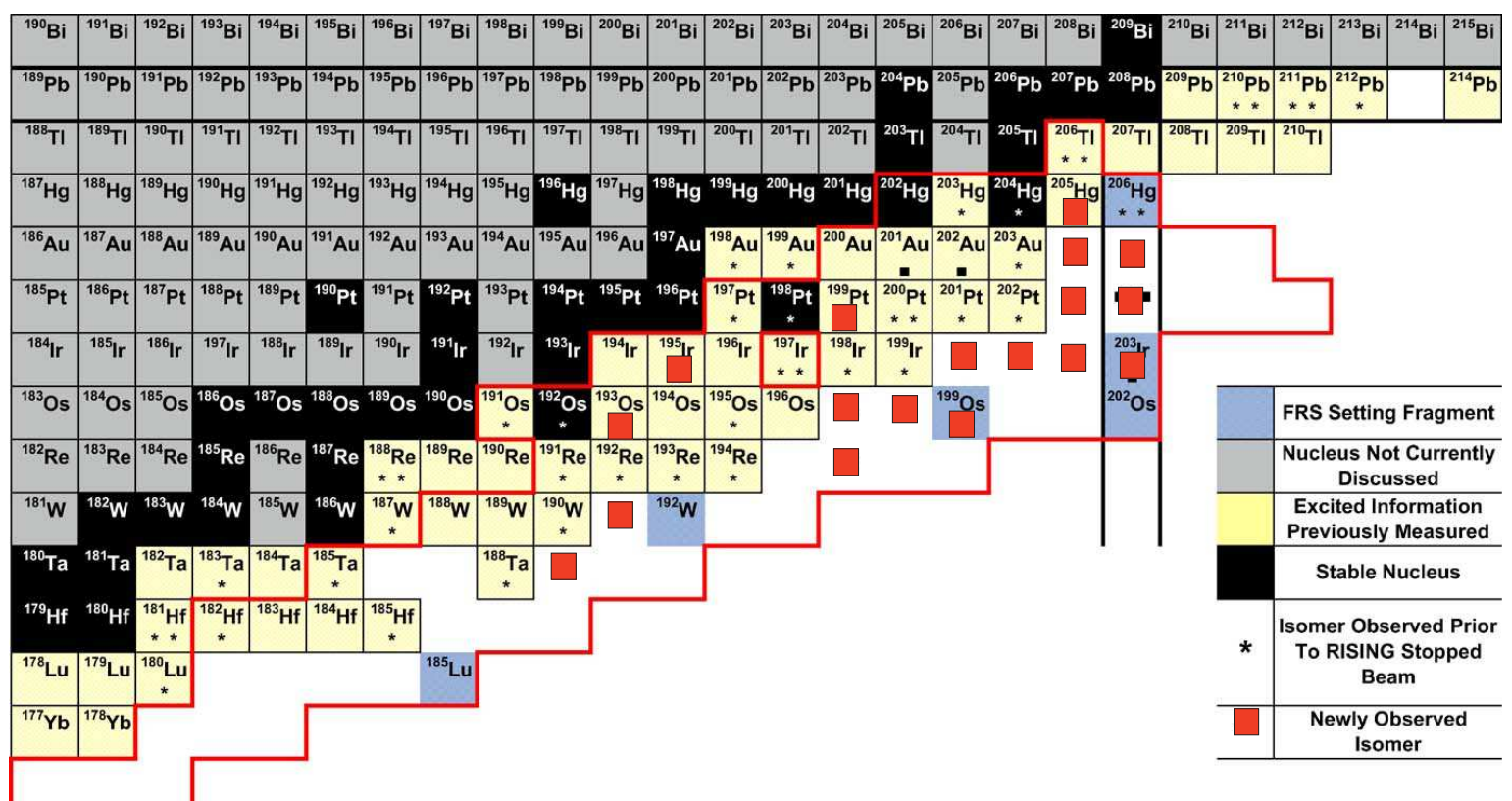

Figure 23. Isomers studied with projectile fragmentation, from Steer et al. [416]. The red solid line indicates the region explored with several settings of the FRS.

nuclei, resulting in the discovery of numerous isomers [416]. Studies of the high-spin population in terms of isomeric ratios indicate more favouring of higher-spins than expected $[412,420]$, reaching spins of $\leq 55 / 2 \hbar$.

RIKEN is currently provding the highest-intensity projectile fragmentation and fission products, with many new isomers being discovered [421], although the lower beam energies (for example, $345 \mathrm{MeV} /$ nucleon ${ }^{238} \mathrm{U}$ ) favour lower- $Z$ values for unambiguous identifications. Following the incorporation of RISING germanium detectors as part of the EURICA collaboration, it has been possible to identify isomers in the most exotic nuclei, such as ${ }^{138} \mathrm{Sn}$ [381], and reaching $Z$ values up to 64 in the discovery of isomers in ${ }^{164} \mathrm{Sm}$ and ${ }^{166} \mathrm{Gd}[382]$.

10.2.2. Incomplete fusion reactions The well-known and long-exploited fusionevaporation reactions with stable beams lead naturally to neutron-deficient nuclei. In the quest to study high-spin states in stable and slightly neutron-rich nuclei, significant advances were made with incomplete-fusion reactions (see Ref. [422] and Refs. therein) such as $\left({ }^{7} \mathrm{Li}, \alpha x n\right),\left({ }^{9} \mathrm{Be}, \alpha x n\right)$ and $\left({ }^{11} \mathrm{~B}, \alpha x n\right)$. For example, valuable data were obtained for ${ }^{212} \mathrm{Po}$ [165], ${ }^{180} \mathrm{Ta}$ [423], ${ }^{178} \mathrm{Hf}$ [73] and ${ }^{176} \mathrm{Lu}$ [424]. This technique has a relatively limited range of applicability, and further progress has largely depended on radioactive beams and multi-nucleon transfer reactions, as discussed in the accompanying sections.

10.2.3. Fusion-evaporation reactions with radioactive beams The advent of even relatively light neutron-rich beams such as ${ }^{6} \mathrm{He},{ }^{8} \mathrm{He},{ }^{10} \mathrm{Be}$ etc., from new radioactivebeam facilities, and the high cross sections in fusion-evaporation reactions, make it 
possible to study neutron-rich nuclei even with low beam intensities. Unfortunately such reactions will not be as effective as those with stable beams for making highspin states, and therefore high-spin isomers, because the low binding energies of the projectiles (such as ${ }^{8} \mathrm{He}$ ) result in high excitation energies in the compound nucleus, with the consequence that neutrons are evaporated more readily. The net result is to shift the optimum energies for, say, $\left({ }^{8} \mathrm{He}, 4 \mathrm{n}\right)$ reactions on heavy nuclei to about $25 \mathrm{MeV}$, much closer to the Coulomb barrier and therefore with much lower angular momentum input than in typical $\left({ }^{4} \mathrm{He}, 4 \mathrm{n}\right)$ reactions, which peak near $40 \mathrm{MeV}$. Beams of ${ }^{8} \mathrm{He}[425]$ and ${ }^{17} \mathrm{~N}$ [426], among a number developed, have been used, albeit with limited success and with significant challenges being posed by the residual activity presented by the beam particles.

Looking to the longer term, inverse reactions with heavy, neutron-rich beams (such as ${ }^{144} \mathrm{Xe}$, for example) offer the possibility of reaching neutron-rich product nuclei at high spin, and these may ultimately give detailed information about, for example, high- $K$ isomers in neutron-rich hafnium and tungsten isotopes.

10.2.4. Multi-nucleon transfer and deep-inelastic reactions Early studies have shown that reactions with heavy-ion beams with energies of about $6 \mathrm{MeV} /$ nucleon, not far above the Coulomb barrier, were capable of causing significant mass and energy transfer ([427, 428] and references therein). These reactions that began to be exploited in the early 90s $[429,430,431]$ are able to populate a broad range of excited nuclei, albeit in a non-selective way, through the (two-way) transfer of mass and charge, leading to significant excitation energy and angular momentum in the projectile-like and targetlike products [432]. Depending on the energy above the Coulomb barrier, there may well be more than a hundred products in any given reaction, made with progressively lower cross section as more particles are transferred, falling by about an order of magnitude for each successive particle.

Many studies over the last decade have continued to exploit this type of reaction, particularly given the advent of modern $\gamma$-ray arrays, giving access to the study of both projectile-like and target-like products. Coincidence $\gamma$-ray-fragment techniques have been used to provide $Z$ and $A$ identification of the projectile-like products with an appropriate recoil device, PRISMA for example Ref. [433] (see also Ref. [434] for neutron-rich medium-mass cases). Such measurements can also be used to identify transitions in the complementary heavy products. Although thin-target measurements are not usually appropriate for isomer studies, once some transitions are known the information can be subsequently exploited in thick-target type measurements. Less selective measurements are also possible with devices such as $C H I C O$ installed in GAMMASPHERE, where coarse identification of projectile-like and target-like fragments is possible, allowing selection on grazing-like collisions but still maintaining some sensitivity to isomers (e.g. [435]). Measurements that have signalled the opening up of the landscape for the identification of isomers in well-deformed nuclei, include studies of various lutetium, hafnium, tantalum and tungsten isotopes, carried out mainly 
by the Surrey and Argonne groups and collaborators [436, 437, 438, 439, 440, 441, 442].

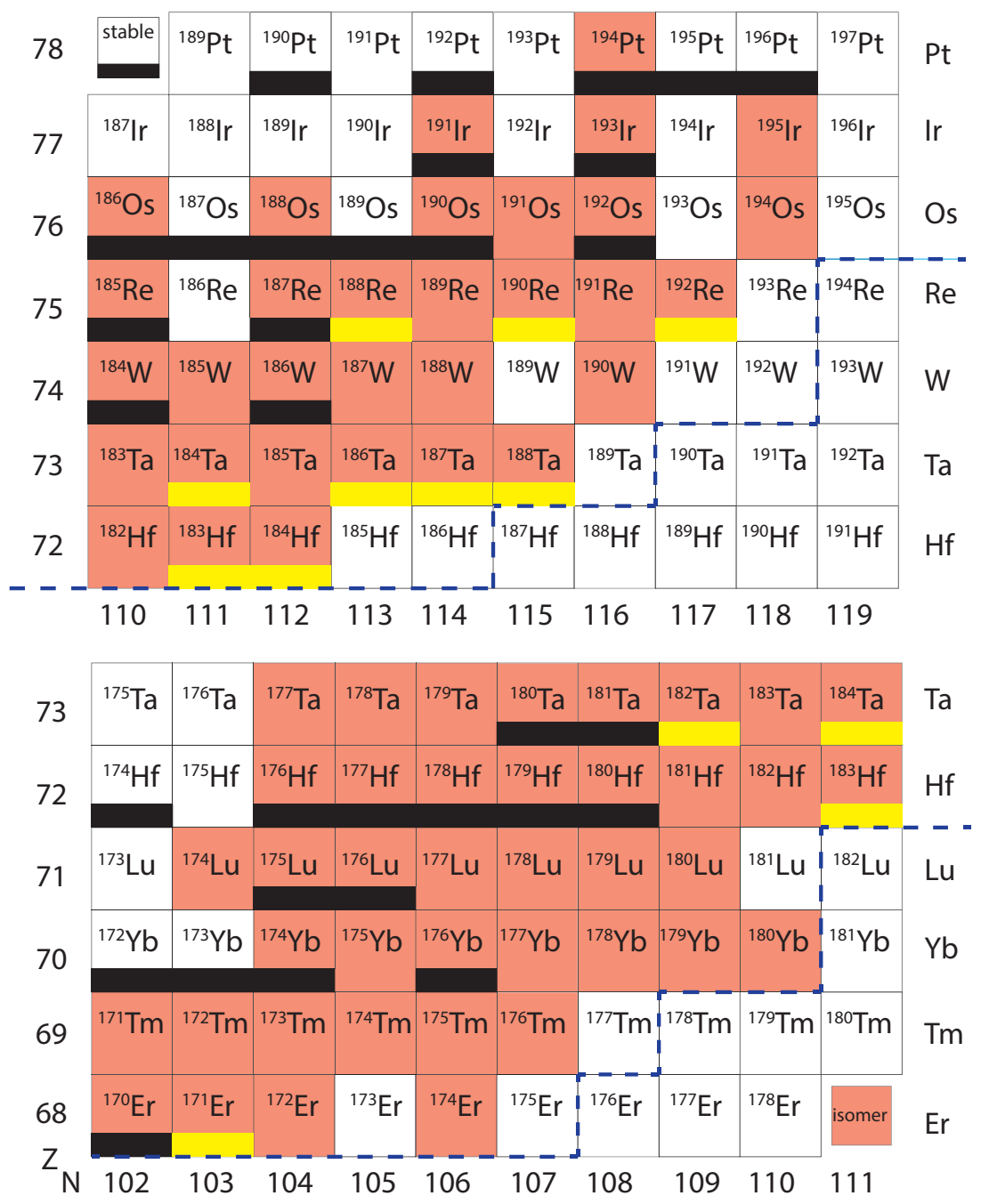

Figure 24. Approximate reach (dashed line) of deep-inelastic reactions with current detector sensitivities in the well-deformed region, updated from Ref. [42]. Nuclides in which new isomers have been identified, or significant information on existing isomers obtained, are indicated by the pink squares. Black bars refer to stable nuclides, and yellow bars refer to nuclides where isomer data are still under evaluation. References are given in the main text. See also Kondev et al. [41].

A systematic set of measurements have been carried out by a Canberra-Argonne collaboration, using similar conditions for each, with $\sim 6 \mathrm{MeV} /$ nucleon ${ }^{136} \mathrm{Xe}$ beams on a variety of the most neutron-rich stable targets available. All have exploited time correlations with GAMMASPHERE and a variety of beam pulsing and chopping. The measurements have led to extensive new information on isomers in a range of nuclei near stability and also somewhat neutron-rich, as depicted schematically in figure 24 . Analysis of these data continues but details of completed results are published in a series of papers [33, 67, 74, 94, 126, 128, 129, 130, 443, 444, 445, 446, 447, 448, 449, 450, 451]. They cover a broad range in terms of physics, from the characterisation of comprehensive 
multi-quasiparticle isomers in ${ }^{174} \mathrm{Yb}$ [445], for example, to the identification of transitions to intermediate states that could connect the high- $K$ and low- $K$ isomeric and ground states in ${ }^{176} \mathrm{Lu}$ by photoexcitation, thus affecting elemental abundances in a stellar environment [33].

Comparing the scope of these measurements with those obtained from the fragmentation studies discussed in the preceding section, the deep-inelastic approach generally reaches to about four neutrons beyond stability, considerably less than fragmentation, although with more extensive spectroscopic information being extracted, a function partly of the $\gamma$-ray detection systems currently available and yields. The approaches should be seen as complementary, since $Z$ and $A$ identification is limited in thick-target deep-inelastic studies, but unambiguous assignments are usually possible in fragmentation.

10.2.5. Isomers in storage rings Early measurements in storage rings were able to resolve $\beta$-decaying isomers in the mass-50 region [452]. More recent mass measurements exploiting time-resolved Schottky mass spectroscopy [453] in the ESR [454] have demonstrated a sensitivity down to single ions, and a precision of about $10 \mathrm{keV}$ for locating highly-excited isomeric states in relatively heavy nuclei, opening up new prospects for identifying low-yield exotic states [82, 455, 456]. At present, the crucial cooling stage takes a few seconds, which limits the technique to states with lifetimes down to a few seconds unless the initial yield is very high. The power of the technique is that it is essentially decoupled from the need to observe the decay radiation, although the signatures of the decay processes themselves can be "read" from the pattern of trajectories observed.

This can can be seen from the example of a recent measurement $[82,83]$ shown in figure 25. Ions of exotic isotopes were produced using fragmentation of a $\sim 500$ $\mathrm{MeV} /$ nucleon gold beam, separated with the FRS and injected into the ESR at GSI. Three sequential injections are shown in the top panel, involving states in fully stripped ${ }^{184} \mathrm{Hf}$ and also H-like ${ }^{184} \mathrm{Ta}$, in a map of the time evolution (running down the page) of Schottky signals as a function of their relative frequency. Essentially four different trajectories can be seen at different times: three associated with states in ${ }^{184} \mathrm{Hf}$ (ground and excited states as marked) and one with the ground state of ${ }^{184} \mathrm{Ta}$, all neutron-rich isotopes.

An important point is that the amplitude of the Schottky signal is directly proportional to the number of ions. The middle panel shows a detail of the third injection (reproduced in the bottom panel): the intensity at the beginning indicates the presence of two ions with the same mass, one of which (lower intensity track) proceeds and terminates at about $150 \mathrm{~s}$, while a second track of lower mass is born at about $20 \mathrm{~s}$ after injection. The signature here is that terminating tracks indicate a $\beta$-decay, leading to a fully stripped ion outside the field-of-view (other decays that produce large recoil momentum may have a similar effect) while the birth of the second track at lower mass indicates the $\gamma$-decay of one isomeric ion to a lower excited state. (Of course, for fully 


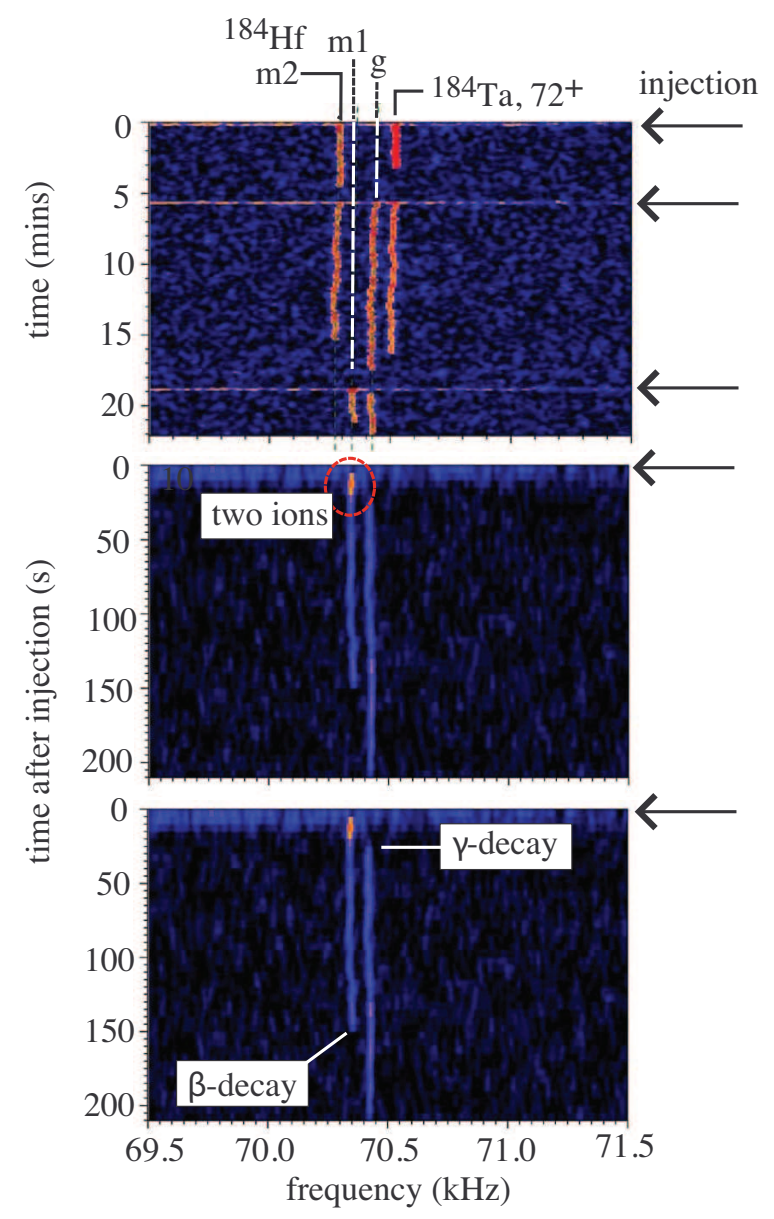

Figure 25. Schottky spectra for circulating $72+$ ions with $A=184[82,83]$. The figure is adapted from Ref. [42]. See text for explanation.

stripped ions internal electron conversion is not possible, so that the measured lifetimes will be correspondingly longer than those when atomic electrons are present, depending on decay paths.)

The $K^{\pi}=8^{-}, m 1$ isomer was originally identified using deep-inelastic reactions and on-line separation at GSI [457]. From that $\gamma$-decay work, the energy was precisely known at $1272.4 \mathrm{keV}$. This provides a good test of the mass measurements which give an excitation energy of $1264(10) \mathrm{keV}$. The higher lying $(\mathrm{m} 2)$ isomer observed at $2477(10)$ $\mathrm{keV}$ has been associated with a $K^{\pi}=15^{+}$state, expected from multi-quasiparticle calculations at about $2369 \mathrm{keV}$ [82]. The four-quasiparticle configuration contains the two-proton $8^{-}$"building block" as a component, together with the $3 / 2^{-}[512]$ and $11 / 2^{+}[615]$ neutrons that lie near the Fermi surface. The lifetime of the upper isomer is consistent with a weak $E 3 \gamma$-ray branch (see Refs. [82, 83] for details) which would proceed through the unknown states of the $K^{\pi}=8^{-}$band, while the $\beta$-decay is likely to pass through a high- $K$, high-spin path, within the excited states of ${ }^{184} \mathrm{Ta}$.

Although the spectroscopic information obtained with these measurements is very limited, and there will be ambiguities in relating the isomers to the spectrum of 
calculated states, since not all calculated states will necessarily lead to isomers in the accessible lifetime region, once some states are known, experimental studies tailored to observe the subsequent decays become feasible. This is an exciting prospect given that little, if anything, is known about these nuclei. Preliminary studies have already commenced at ISOLDE and more are planned for the future building on the established capabilities for producing neutron-rich hafnium beams [458].

Similar prospects exist for other cases such as the two isomers identified in ${ }^{187} \mathrm{Ta}$ with suggested spins of $27 / 2^{+}\left(1789 \mathrm{keV}, T_{1 / 2}=22(9) \mathrm{s}\right)$ and $41 / 2^{+}\left(2935 \mathrm{keV}, T_{1 / 2}\right.$ $>5$ min.). Gamma-ray decays into ${ }^{187} \mathrm{Ta}$ itself should reveal low- and high-seniority high-spin states in that nucleus, while the $\beta$-decays from both isomers provide a similar window into ${ }^{187} \mathrm{~W}$. The $\beta$-decay paths are forced to go to high- $K$ daughter states since only specific one-particle decays will proceed.

These developments can be viewed in the tradition of high-spin studies using radioactive sources of isomeric states, such as the 160 -day $K^{\pi}=23 / 2^{-}$isomer in ${ }^{177} \mathrm{Lu}$ whose decay exposed high- $K$ structures in both ${ }^{177} \mathrm{Lu}$ and in the $\beta$-decay daughter ${ }^{177} \mathrm{Hf}$ [459], and is still being exploited [460]. The much shorter lifetimes involved in the neutron-rich nuclei might not be usable for the production of exotic sources but they might yet find applications as (weak) exotic beams (samples) that can be studied themselves, or be used to study reactions. This is one of the expected applications for the programme of experiments when a storage ring is installed at ISOLDE [461].

\subsection{Sub-nanosecond timing detectors: $\mathrm{LaBr}_{3}$}

New arrays of $\mathrm{LaBr}_{3} \gamma$-ray detectors are now making their mark in the determination of half-lives $\gtrsim 50 \mathrm{ps}$. Typically, the detectors are incorporated into arrays of germanium detectors, to benefit from the superior germanium energy resolution, such as in ROSPHERE in Romania [462, 463], GAMMASPHERE in the USA [464], EXILL in France [465, 466], and EURICA in Japan [467].

Although germanium detectors can already be used to determine half-lives in the $1 \mathrm{~ns}$ region, this is rarely achieved in multi-detector arrays, so that the inclusion of $\mathrm{LaBr}_{3}$ detectors represents a significant advance. As with longer-lived states, the subnanosecond timing capability has two specific benefits: (i) the experimental ability to establish the temporal ordering of transitions [41], and (ii) the ability to determine short half-lives for the purpose of evaluating hindrance factors and their structure implications. For example, the $644 \mathrm{keV}, 5^{-} \rightarrow 4^{+} E 1$ transition in ${ }^{136}$ Ce has been measured to have a half-life of 496(23) ps [463], yielding a transition strength of $1.6(3) \times 10^{-6}$ W.u., which is interpreted as indicating no significant $K$ hindrance. Further measurements of this kind will help to determine the limiting features of the $K$ quantum number, with many candidate intrinsic states that have only upper-limit half-lives tabulated by Kondev et al. [41]. 


\subsection{Separating isomers from ground states}

In the earlier sections, there has been emphasis on using decay properties to enable isomer-selective investigations. In this section, we concentrate on the use of other isomer properties that enable them to be separated physically from their respective ground states, with the possibility of preparing pure isomeric beams. The key properties exploited here are masses and magnetic dipole moments. The associated techniques for isomer-beam preparation have not yet been fully developed, but they will become increasingly important as the new generation of radioactive-beam accelerators comes on-line.

10.4.1. Mass selection A mass resolution of better than 1 part in $10^{5}$ is one avenue for achieving physical isomer separation, and this can be provided by storage rings (Sect. 10.2.5) and Penning traps. In the former case, using the ESR at GSI, it is possible to insert a mechanical scraper to remove unwanted ions. While proof-of-principle for this has been demonstrated [468], it remains to be used in practice to generate a purified isomer beam. In addition, there are cases where an isomer is longer lived than its respective ground state. For example, the $J^{\pi}=18^{+}$isomer of ${ }^{210} \mathrm{Po}$ has a half-life of $45 \mathrm{~s}$, whereas the ground state has a half-life of $0.3 \mu \mathrm{s}$. Therefore, after a short storage time the ground state will have decayed and the isomer would be purified.

Penning traps are widely used for accurate mass measurements of radioisotopes [469]. In common with storage rings, Penning traps can detect isomers without the need for decay radiations, such as in the discovery of an isomer in ${ }^{65} \mathrm{Fe}$ [470] at the LEBIT facility at NSCL. Furthermore, with the ability to eject ions from the trap in a mass selective manner, it is possible to perform spectroscopy on the selected radioisotope. Indeed, isomer selection has been demonstrated with ${ }^{100} \mathrm{Nb}$ [471] at JYFLTRAP, Jyväskylä, and with thallium isomers [472] at ISOLTRAP, CERN. A further recent development capable of fast $(\sim 10 \mathrm{~ms})$ separation of isomers from ground states is that of multi-reflection time-of-flight (MR-ToF) mass spectrometry $[473,474,475]$, now successfully demonstrated with the $J^{\pi}=25 / 2^{+},{ }^{211}$ Po isomer [476].

10.4.2. Hyperfine structure Laser techniques exploit differences in hyperfine structure [477] such that an isomer can be distinguished from its corresponding ground state, not only without the need for decay radiations, but also without the need for a significant mass difference. Thus, in the case of ${ }^{80} \mathrm{Ga}$, measurements at ISOLDE enabled the discovery of a new isomer [478], whose excitation energy remains unknown but whose decay properties have since been studied in detail [479].

With an $I S O L$ facility, the ionisation stage can be implemented with in-source laser resonant ionisation. The ionisation efficiency is frequency dependent and sensitive to the hyperfine structure, especially that arising from the nuclear magnetic dipole moment. Since different chemical elements present different atomic structures, element-specific schemes for resonant ionisation need to be established. A very successful case has been 
the resonant ionisation of copper at ISOLDE, enabling, for example, the separation and reacceleration of the $J^{\pi}=66^{-}$isomer of ${ }^{68} \mathrm{Cu}$ for Coulomb excitation studies [366].

In-source resonant ionisation has limitations due to the thermal broadening of the hyperfine structure. Improved resolution can be achieved with the in-flight collinear technique [477], which has recently been developed at ISOLDE and successfully applied to francium isotopes and isomers [121, 480], including isomer-selected $\alpha$-coincidence spectroscopy.

In a separate development, the hyperfine structure of highly charged ions can be exploited in storage rings, through the process of dielectronic recombination, to separate isomers from ground states [481].

\section{Open questions and closing comments}

As discussed above, isomers can be used as probes of nuclear reactions and nuclear structure, with an essentially unlimited scope. In themselves, isomers are not necessarily the focal point of interest: after all, there are likely to be more isomers than there are nuclear species. As well as the possible applications that might eventuate, the primary interest arises, in our view at least, from the fact that isomerism can be indicative of unusual/unexpected nuclear structure and it can also, simultaneously, provide a means of characterising that structure. Isomers thus provide a highly sensitive tool whose utility is not decoupled from the primary imperatives of the discovery and study of exotic nuclei.

Within this context, the preceding sections have addressed a number of unresolved issues, not the least of which is the extent to which isomers might provide additional stability, or at least longer half-lives, at the outer reaches of the nuclear chart. There is the prospect here of a different perspective in the way that we view the limits to nuclear binding. To appreciate this possibility, it is necessary to have a better understanding of the controlling factors in isomer decay rates, but the theory of transition probabilities, for transitions that are essentially forbidden, is not yet well developed. When it comes to isomeric fission, the gap in understanding seems to widen even further, and here the empirical knowledge is also rudimentary.

A better understanding of isomer decay rates would surely also improve the prospects for isomer manipulation and control, with concomitant applications. From a societal perspective, this is an immense challenge. It seems to require not only interdisciplinary breakthroughs, but also the chance occurrence of an appropriate juxtaposition of nuclear states. The relationship between steady progress and serendipity is complex.

Significant progress has been made in developing reactions that can (i) access neutron-rich heavy nuclei, (ii) more generally reach further towards the outer reaches of the domain of nuclear binding, and (iii) at the same time provide isomeric beams with novel capabilities. While these advances have already been highly productive, they are far from being fully developed in terms of production and detection techniques, or 
exhausted in terms of their discovery potential. Furthermore, new accelerator facilities are currently under construction or being planned, and their exploitation will include studies of nuclear isomers. There is always a problem to be aware of with the study of isomers though, and that is that the popular techniques for their identification can be compromised if the lifetimes become very long, as might occur, paradoxically, in the more interesting cases.

\section{Acknowledgments}

Special thanks for their physics input go to Bogdan Fornal, Greg Lane, Matt Reed, and Furong Xu. George Dracoulis received support from the Australian Research Council Discovery programme. Phil Walker is supported by the UK Science and Technology Facilities Council under grant No. ST/L005743/1. Filip Kondev is supported by the U.S. Department of Energy, Office of Science, Office of Nuclear Physics under contract No. DE-AC02-06CH11357.

\section{References}


[1] Hahn O 1921 Über eine neue radioaktive Substanz im Uran Chem. Ber. 54 1131-42

[2] Soddy F 1917 The complexity of the chemical elements Nature 99 433-8

[3] Gamow G 1934 Negative Protons and Nuclear Structure Phys. Rev. 45 728-29

[4] von Weizsäcker C F 1936 Metastabile Zustände der Atomkerne Naturewissenschaften 24 813-814

[5] Segrè E and Hemholz A C 1949 Nuclear isomerism Rev. Mod. Phys. 21 271-305

[6] Goldhaber M and Hill R D 1952 Nuclear isomerism and shell structure Rev. Mod. Phys. 24 179-239

[7] Goldhaber M and Weneser J 1955 Electromagnetic transitions in nuclei Ann. Rev. Nuc. Sci. 5 $1-24$

[8] Goldhaber M and Sunyar A W 1951 Classification of nuclear isomers Phys. Rev. 83 906-18

[9] Bohr A and Mottelson B R 1953 Rotational states in even-even nuclei Phys. Rev. 90 717-19

[10] Burson S B, Blair K W, Keller H B and Wexler S 1951 The radiations from hafnium Phys. Rev. 83 62-8

[11] Bohr A and Mottelson B R 1953 Interpretation of isomeric transitions of electric quadrupole type Phys. Rev. 89 316-17

[12] Bohr A and Mottelson B R 1953 Collective and individual-particle aspects of nuclear structure K. Dan. Vidensk. Selsk. Mat. Fys. Medd. 27 nr. 16

[13] Bohr A and Mottelson B R 1955 Moments of inertia of rotating nuclei K. Dan. Vidensk. Selsk. Mat. Fys. Medd. 30 nr. 1

[14] Alaga G, Alder K, Bohr A and Mottelson B R 1955 Intensity rules for beta and gamma transitions to nuclear rotational states K. Dan. Vidensk. Selsk. Mat. Fys. Medd. 29 nr. 9

[15] Bohr A 1976 Rotational motion in nuclei Rev. Mod. Phys. 48 365-74

[16] Bohr A and Mottelson B R 1981 Single-Particle and Collective Aspects of Nuclear Rotation Phys. Scr. 24 71-76

[17] Walker P M and Dracoulis G D 1999 Energy traps in atomic nuclei Nature 399 35-40

[18] Neyens G 2003 Nuclear magnetic and quadrupole moments for nuclear structure research on exotic nuclei Rep. Prog. Phys. 66 633-689; Erratum Rep. Prog. Phys. 661251

[19] Becker J A 2006 Atomic-Nuclear Coupling Experiments AIP Conf. Proc. 819 396-401

[20] Matinyan S 1998 Lasers as a bridge between atomic and nuclear physics Phys. Rep. 298 199-249

[21] Gobet F, Plaisir C, Hannachi F, Tarisien M, Bonnet T, Versteegen M, Aléonard M M, Gosselin G, Méot V and Morel P 2011 Nuclear physics studies using high energy lasers Nucl. Instr. Meth. Phys. Res. A653 80-83

[22] Pálffy A, Evers J and Keitel C H 2007 Isomer triggering via nuclear excitation by electron capture Phys. Rev. Lett. 99 172502

[23] Walker P M and Carroll J J 2005 Ups and downs of nuclear isomers Phys. Today 58 39-44

[24] NSAC Isotopes Subcommittee 2009 Isotopes for the Nation's Future: A long range plan, August 27, 2009

[25] Nuclear Physics for Medicine 2014 Report from the Nuclear Physics European Collaboration Committee eds. F. Azaiez, A. Bracco, J. Dobes, A. Jokinen, G-E. Körner, A. Maj, A. Murphy and P. Van Duppen

[26] Langanke K and Wiescher M 2001 Nuclear reactions and stellar processes Rep. Prog. Phys. 64 $165 \%-701$

[27] Arnould M, Goriely S and Takahashi K 2007 The r-process of stellar nucleosynthesis: Astrophysics and nuclear physics achievements and mysteries Phys. Rep. 450 97-213

[28] Arnould M and Goriely S 2003 The p-process of stellar nucleosynthesis: astrophysics and nuclear physics status Phys. Rep. 384 1-84

[29] Belic D, Arlandini C, Besserer J, de Boer J, Carroll J J, Enders J, Hartmann T, Käppeler F, Kaiser H, Kneissl U, Loewe M, Maier H J, Maser H , Mohr P, von Neumann-Cosel P, Nord A, Pitz H H, Richter A, Schumann M, Volz S and Zilges A 1999 Photoactivation of ${ }^{180} \mathrm{Ta}^{m}$ and its implications for the nucleosynthesis of nature's rarest naturally occurring isotope Phys. Rev. Lett. 83 5242-45 
[30] Belic D, Arlandini C, Besserer J, de Boer J, Carroll J J, Enders J, Hartmann T, Käppeler F, Kaiser H, Kneissl U, Kolbe E, Langanke K, Loewe M, Maier H J, Maser H, Mohr P, von Neumann-Cosel P, Nord A, Pitz H H, Richter A, Schumann M, Thielemann F -K, Volz S and Zilges A 2002 Photo-induced depopulation of the ${ }^{180} \mathrm{Ta}^{m}$ isomer via low-lying intermediate states: Structure and astrophysical implications Phys. Rev. C 65035801

[31] Walker P M, Dracoulis G D and Carroll J J 2001 Interpretation of the excitation and decay of ${ }^{180} \mathrm{Ta}^{m}$ through a $\mathrm{K}^{\pi}=5^{+}$band Phys. Rev. C 64 061302(R)

[32] Dracoulis G D 2010 On the question of connections between high-K and low-K states in ${ }^{180} \mathrm{Ta}$ and ${ }^{176} \mathrm{Lu}$ AIP Conf. Proc. 1269 295-302

[33] Dracoulis G D, Kondev F G, Lane G J, Byrne A P, Carpenter M P, Janssens R V F, Lauritsen T, Lister C J, Seweryniak D and Chowdhury P 2010 Connections between high-K and low-K states in the s-process nucleus ${ }^{176} \mathrm{Lu}$ Phys. Rev. C81 011301(R)

[34] Mohr P, Käppeler F and Gallino R 2007 Survival of nature's rarest isotope ${ }^{180} \mathrm{Ta}$ under stellar conditions Phys. Rev. C 75 012802(R)

[35] Gintautas V, Champagne A E, Kondev F G and Longland R 2009 Thermal equilibration of ${ }^{176} \mathrm{Lu}$ via K mixing Phys. Rev. C80 015806

[36] de Voigt M J A, Dudek J and Szymanski S 1983 High-spin phenomena in atomic nuclei Rev. Mod. Phys. 55 949-1046

[37] Walker P M and Dracoulis G D 2001 Exotic Isomers in Deformed Atomic Nuclei Hyperfine Interact. 135 83-107

[38] Heyde K and Wood J L 2011 Shape coexistence in atomic nuclei Rev. Mod. Phys. 83 1467-521

[39] Aprahamian A and Sun Y 2005 Long live isomer research Nature Phys. 1 81-2

[40] Walker P M and Carroll J J 2007 Nuclear isomers: recipes from the past and ingredients for the future Nucl. Phys. News 17 11-15

[41] Kondev F G, Dracoulis G D and Kibédi T 2015 Configurations and hindered decays of K isomers in deformed nuclei with A>100 At. Data Nucl. Data Tables 103-104 50-105; Erratum At. Data Nucl. Data Tables 105-106 105-106

[42] Dracoulis G D 2013 Isomers, nuclear structure and spectroscopy Phys. Scr. T152 014015

[43] Walker P M and Xu F R 2016 High-K isomerism in rotational nuclei Phys. Scr. 91013010

[44] Audi G, Kondev F G, Wang M, Pfeiffer B, Sun X, Blachot J and MacCormick M 2012 The NUBASE2012 evaluation of nuclear properties Chinese Phys. C36 1157-286

[45] Jain A K, Maheshwari B, Garg S, Patial M and Singh B 2015 Atlas of nuclear isomers Nucl. Data Sheets $1281-130$

[46] Stone N J 2014 Table of nuclear magnetic dipole and electric quadruple moments IAEA Nuclear Data Section Report INDC(NDS)-0658, IAEA, Vienna, Austria

[47] Evaluated Nuclear Structure Data File (ENSDF) and Unevaluated Nuclear Data List (XUNDL), www.nndc.bnl.gov/ensdf

[48] de Shalit A and Talmi I 1963 Nuclear Shell Theory (Academic Press, NY) p. 315

[49] Lawson R D 1980 Theory of the Nuclear Shell Model (Clarendon, Oxford)

[50] Rowe D J and Wood J L 2010 Fundamentals of Nuclear Models (World Scientific, Singapore)

[51] Mayer R H, Nisius D T, Bearden I G, Bhattacharyya P, Richter L, Sferrazza M, Grabowski Z W, Daly P J, Broda R, Fornal B, Ahmad I, Carpenter M P, Henry R G, Janssens R V F, Khoo T L, Lauritsen T, Liang Y and Blomqvist J 1994 Phys. Lett. B336 308-12

[52] Zhang C T, Bhattacharyya P, Daly P J, Grabowski Z W, Broda R, Fornal B and Blomqvist J 2000 Yrast isomers of $\left(\nu \mathrm{h}_{11 / 2}\right)^{n}$ character in ${ }^{125} \mathrm{Sn}$ and ${ }^{126} \mathrm{Sn}$ Phys. Rev. C 62 057305

[53] Lozeva R L, Simpson G S, Grawe H, Neyens G, Atanasova L A, Balabanski D L, Bazzacco D, Becker F, Bednarczyk P, Benzoni G, Blasi N, Blazhev A, Bracco A, Brandau C, Caceres L, Camera F, Chamoli S K, Crespi F C L, Daugas J -M, Detistov P, De Rydt M, Doornenbal P, Fahlander C, Farnea E, Georgiev G, Gerl J, Gladnishki K A, Górska M, Grebosz J, Hass M, Hoischen R, Ilie G, Ionescu-Bujor M, Iordachescu A, Jolie J, Jungclaus A, Kmiecik M, Kojouharov I, Kurz N, Lakshmi S P, Lo Bianco G, Mallion S, Maj A, Montanari D, Perru 
O, Pfützner M, Pietri S, Pinston J A, Podolyák Zs, Prokopowicz W, Rudolph D, Rusev G, Saitoh T R, Saltarelli A, Schaffner H, Schwengner R, Tashenov S, Turzó K, Valiente-Dobón J J, Vermeulen N, Walker J, Werner-Malento E, Wieland O and Wollersheim H -J 2008 New sub- $\mu$ s isomers in ${ }^{125,127,129} \mathrm{Sn}$ and isomer systematics of ${ }^{124130} \mathrm{Sn}$ Phys. Rev. C 77064313

[54] Ressler J J, Casten R F, Zamfir N V, Beausang C W, Cakirli R B, Ai H, Amro H, Caprio M A, Hecht A A, Heinz A, Langdown S D, McCutchan E A, Meyer D A, Plettner C, Regan P H, Sciacchitano M J S and Yamamoto A D 2004 Transition from the seniority regime to collective motion Phys. Rev. C69 034317

[55] Lawson R D $1981\left(\pi \mathrm{h}_{11 / 2}\right)^{n}$ states expected in ${ }_{68}^{150} \operatorname{Er}_{82},{ }_{69}^{151} \mathrm{Tm}_{82}$ and ${ }_{70}^{152} \mathrm{Yb}_{82}$ Z. Phys. A303 $51-61$

[56] McNeill J H, Blomqvist J, Chishti A A, Daly P J, Gelletly W, Hotchkis M A C, Piiparinen M, Varley B J and Woods P J 1989 Exotic N=82 nuclei ${ }^{153} \mathrm{Lu}$ and ${ }^{154} \mathrm{Hf}$ and filling of the $\pi \mathrm{h}_{11 / 2}$ subshell Phys. Rev. Lett. 63 860-3

[57] McNeill J H, Blomqvist J, Chishti A A, Daly P J, Gelletly W, Hotchkis M A C, Piiparinen M, Varley B J and Woods P J 1993 Isomeric decay studies using a recoil mass separator Z. Phys. A344 369-79

[58] Broda R, Fornal B, Krolas W, Pawkat T, Bazzacco D, Lunardi S, Rossi-Alvarez C, Menegazzo R, de Angelis G, Bednarczyk P, Rico J, De Acuna D, Daly P J, Mayer R H, Sferrazza M, Grawe H, Maier K H and Schubart R 1995 N=40 neutron subshell closure in the ${ }^{68} \mathrm{Ni}$ nucleus Phys. Rev. Lett. 74 868-71

[59] Mazzocchi C, Grzywacz R, Batchelder J C, Bingham C R, Fong D, Hamilton J H, Hwang J K, Karny M, Krolas W, Liddick S N, Lisetskiy A F, Morton A C, Mantica P F, Mueller W F, Rykaczewski K P, Steiner M, Stolz A and Winger J A 2005 Low energy structure of even-even $\mathrm{Ni}$ isotopes close to ${ }^{78} \mathrm{Ni}$ Phys. Lett. B622 45-54

[60] Chiara C J, Walters W B, Stefanescu I, Alcorta M, Carpenter M P, Fornal B, Gürdal G, Hoffman C R, Janssens R V F, Kay B P, Kondev F G, Królas W, Lauritsen T, Lister C J, McCutchan E A, Pawłat T, Rogers A M, Seweryniak D, Sharp N, Wrzesiński J and Zhu S 2011 Seniority, collectivity, and B(E2) enhancement in ${ }^{72} \mathrm{Ni}$ Phys. Rev. C84 037304

[61] Van Isacker P and Heinze S 2008 Partial conservation of seniority and nuclear isomerism Phys. Rev. Lett. 100052501

[62] Van Isacker P 2011 Seniority isomers in nuclei J. Phys. Conf. Ser. 322 012003

[63] Stephens F S 1975 Coriolis effect and rotational alignment in nuclei Rev. Mod. Phys. 47 43-65

[64] Kondev F G, Dracoulis G D, Byrne A P and Kibédi T 1998 Intrinsic states and rotational bands in ${ }^{176} \mathrm{Ta}$ and ${ }^{178} \mathrm{Ta}$ Nucl. Phys. A632 $473-539$

[65] Löbner K E G 1968 Systematics of absolute transition probabilities of K-forbidden gamma-ray transitions Phys. Lett. B26 369-70

[66] Dracoulis G D, Walker P M and Kondev F G 1998 Pairing reduction and rotational motion in multi-quasiparticle states Phys. Lett. B419 7-13

[67] Dracoulis D G, Lane G J, Kondev F G, Byrne A P, Hughes R O, Nieminen P, Watanabe H, Carpenter M P, Janssens R V F, Lauritsen T, Seweryniak D, Zhu S, Chowdhury P and Xu F R 2006 Two-quasiparticle Kisomers and pairing strengths in the neutron-rich isotopes ${ }^{174} \mathrm{Er}$ and ${ }^{172}$ Er Phys. Lett. B635 200-6

[68] Tandel S K 2014 K-isomers as probes of nuclear structure AIP Conf. Proc. 1609 157-60

[69] Dracoulis G D, Byrne A P, Baxter A M, Davidson P M, Kibédi T, McGoram T R, Bark R A and Mullins S M 1999 Spherical and deformed isomers in ${ }^{188} \mathrm{~Pb}$ Phys. Rev. C60 014303

[70] Dracoulis G D, Lane G J, Byrne A P, Kibédi T, Baxter A M, Macchiavelli A O, Fallon P and Clark R M 2004 Spectroscopy of ${ }^{188} \mathrm{~Pb}$ : evidence for shape coexistence Phys. Rev. C69 054318

[71] de Boer F W N, Goudsmit P F A, Meijer B J, Kapteyn J C, Konijn J and Kamermans R 1976 The four quasi-particle ${ }^{178} \mathrm{Hf}$ isomeric state, its excitation energy and multipolarities of deexciting transitions Nucl. Phys. A263 397-409

[72] Khoo T L and Løvhøiden G 1977 Structural Changes in the Yrast States in ${ }^{178}$ Hf Phys. Lett. B67 
$271-4$

[73] Mullins S M, Dracoulis G D, Byrne A P, McGoram T R, Bayer S, Seale W A and Kondev F G 1997 Rotational band on the 31 yr $16^{+}$isomer in ${ }^{178} \mathrm{Hf}$ Phys. Lett. B393 279-84; Erratum Phys. Lett. B400 401

[74] Dracoulis G D, Lane G J, Kondev F G, Watanabe H, Seweryniak D, Zhu S, Chiara C J, Carpenter M P, Janssens R V F, Lauritsen T, Lister C J, McCutchan E and Stefanescu I 2009 Lifetime of the $\mathrm{K}^{\pi}=8^{-}$isomer in ${ }^{174} \mathrm{Er}$ and $\mathrm{E} 1$ systematics Phys. Rev. C79 061303(R)

[75] Walker P M, Dracoulis G D, Byrne A P, Fabricius B, Kibédi T, Stuchbery A E and Rowley N 1994 Multi-quasiparticle and rotational structures in ${ }^{179} \mathrm{~W}$ : Fermi alignment, the K-selection rule and blocking Nucl. Phys. A568 397-444

[76] Jain K, Burglin O, Dracoulis G D, Fabricius B, Rowley N and Walker P M 1995 Multiquasiparticle states in the mass-180 region Nucl. Phys. A591 61-84

[77] Gallagher C J and Moszkowski S A 1958 Coupling of Angular Momenta in Odd-Odd Nuclei Phys. Rev. 111 1282-90

[78] Gallagher C J 1962 Coupling of Angular Momenta in Two-Particle States in Deformed Even-Even Nuclei Phys. Rev. 126 1525-31

[79] Mullins S M, Byrne A P, Dracoulis G D, McGoram T R and Seale W A 1998 High-spin intrinsic and rotational states in the stable nucleus ${ }^{177} \mathrm{Hf}$ : evidence for reaction-dependent spin population Phys. Rev. C58 831-45

[80] Liu H L, Xu F R, Walker P M and Bertulani C A 2011 Deformation and its influence on K isomerism in neutron-rich Hf nuclei Phys. Rev. C 83067303

[81] Åberg S 1978 An investigation of yrast traps in some prolate Hf isotopes Nucl. Phys. A306 89-100

[82] Reed M W, Cullen I J, Walker P M, Litvinov Yu A, Blaum K, Bosch F, Brandau C, Carroll J J, Cullen D M, Deo A Y, Detwiller B, Dimopoulou C, Dracoulis G D, Farinon F, Geissel H, Haettner E, Heil M, Kempley R S, Knöbel R, Kozhuharov C, Kurcewicz J, Kuzminchuk N, Litvinov S, Liu Z, Mao R, Nociforo C, Nolden F, Plass W R, Prochazka A, Scheidenberger C, Steck M, Stöhlker Th, Sun B, Swan T P D, Trees G, Weick H, Winckler N, Winkler M, Woods P J and Yamaguchi T 2010 Discovery of Highly Excited Long-Lived Isomers in NeutronRich Hafnium and Tantalum isotopes through direct mass measurements Phys. Rev. Lett. 105 172501

[83] Reed M W, Walker P M, Cullen I J, Litvinov Yu A, Shubina D, Dracoulis G D, Blaum K, Bosch F, Brandau C, Carroll J J, Cullen D M, Deo A Y, Detwiller B, Dimopoulou C, Dong G X, Farinon F, Geissel H, Haettner E, Heil M, Kempley R S, Knöbel R, Kozhuharov C, Kurcewicz J, Kuzminchuk N, Litvinov S, Liu Z, Mao R, Nociforo C, Nolden F, Plass W R, Podolyak Zs, Prochazka A, Scheidenberger C, Steck M, Stöhlker Th, Sun B, Swan T P D, Trees G, Weick H, Winckler N, Winkler M, Woods P J, Xu F R and Yamaguchi T 2012 Long-lived isomers in neutron-rich Z=72-76 nuclides Phys. Rev. C86 054321

[84] Gjørup N L, Bentley M A, Fabricius B, Holm A, Sharpey-Schafer J F, Sletten G and Walker P M 1990 Seven and Nine Quasi-Particle K-Isomers in ${ }^{175} \mathrm{Hf}$ Z. Phys. A337 353-4

[85] Kondev F G $2005 \mathrm{~K}$-isomers as a probe of nuclear structure and advanced applications AIP Conf. Proc. 769 225-9

[86] Scholes D T, Cullen D M, Kondev F G, Janssens R V F, Carpenter M P, Hartley D J, Djongolov M K, Sletten G, Hagemann G, Wheldon C, Walker P M, Abu Saleem K, Ahmad I, Balabanski D L, Chowdhury P, Danchev M, Dracoulis G D, El-Masri H M, Goon J, Heinz A, Kaye R A, Khoo T L, Lauritsen T, Lister C J, Moore E F, Riedinger L L, Riley M A, Seweryniak D, Shestakova I, Wiedenhöver I, Zeidan O and Zhang J 2004 Highly deformed bands in ${ }^{175} \mathrm{Hf}$ Phys. Rev. C70 054314

[87] Casten R F 1985 A simple approach to nuclear transition regions Phys. Lett. B152 145-50

[88] Walker P M 1990 K-forbidden transitions in the $\mathrm{N}_{p} \mathrm{~N}_{n}$ scheme J. Phys. G16 L233-7

[89] Walker P M and Schiffer K 1991 Interpretation of $\left(\mathrm{h}_{11 / 2}\right)^{2}$ isomers in the $\mathrm{N} \approx 76$ region of transitional nuclei Z. Phys. A338 239-40 
[90] Walker P M 2010 High-spin isomers: structure and applications Nucl. Phys. A834 22c-27c

[91] Walker P M, Lalkovski S and Stevenson P D 2010 Configuration dependence of K-forbidden transition rates from three-quasiparticle isomers Phys. Rev. C81 041304(R)

[92] Swan T P D, Walker P M, Podolyák Zs, Reed M W, Dracoulis G D, Lane G J, Kibédi T and Smith M L 2011 Discovery of a nonyrast $\mathrm{K}^{\pi}=8^{+}$isomer in ${ }^{162} \mathrm{Dy}$, and the influence of competing K-mixing mechanisms on its highly forbidden decay Phys. Rev. C83 034322

[93] Walker P M, Wood R J, Dracoulis G D, Kibédi T, Bark R A, Bruce A M, Byrne A P, Davidson P M, El-Masri H M, Lane G J, Moon C, Orce J N, Prados Estevéz F M, Wheldon C and Wilson A N 2009 Fast decay of a three-quasiparticle isomer in ${ }^{171} \mathrm{Tm}$ Phys. Rev. C79 044321

[94] Dracoulis G D, Kondev F G, Lane G J, Byrne A P, McGoram T R, Kibédi T, Ahmad I, Carpenter M P, Janssens R V F, Lauritsen T, Lister C J, Seweryniak D, Chowdhury P and Tandel S K 2006 Anomalous isomeric decays in ${ }^{174} \mathrm{Lu}$ as a probe of K-mixing and interactions in deformed nuclei Phys. Rev. Lett. 97122501

[95] Walker P M, Dracoulis G D, Byrne A P, Kibédi T and Stuchbery A E $1994 \mathrm{~K}=6^{+}$and $8^{-}$isomer decays in ${ }^{172} \mathrm{Hf}$ and $\Delta \mathrm{K}=8 \mathrm{E} 1$ transition rates Phys. Rev. C49 1718-21

[96] Clark R M, Gregorich K E, Berryman J S, Ali M N, Allmond J M, Beausang C W, Cromaz M, Deleplanque M A, Dragojevic I, Dvorak J, Ellison P A, Fallon P, Garcia M A, Gates J M, Gros S, Jeppesen H B, Kaji D, Lee I Y, Macchiavelli A O, Morimoto K, Nitsche H, Paschalis S, Petri M, Stavsetra L, Stephens F S, Watanabe H and Wiedeking M 2010 High-K Multi-Quasiparticle States in ${ }^{254}$ No Phys. Lett. B690 1924

[97] Walker P M, Cullen D M, Purry C S, Appelbe D E, Byrne A P, Dracoulis G D, Kibédi T, Kondev F G, Lee I Y, Macchiavelli A O, Reed A T, Regan P H and Xu F R 1997 K-forbidden transitions from multi-quasiparticle states Phys. Lett. B408 42-6

[98] Benzoni G, Bracco A, Leoni S, Blasi N, Camera F, Grassi C, Million B, Paleni A, Pignanelli M, Vigezzi E, Wieland O, Matsuo M, Dossing T, Herskind B, Hagemann G B, Wilson J, Maj A, Kmiecik M, Lo Bianco G, Petrache C M, Castoldi M, Zucchiati A, De Angelis G, Napoli D, Bednarczyk P and Curien D 2005 Is the K-quantum number conserved in the order-to-chaos transition region? Phys. Lett. B615 160-6

[99] Leoni S, Benzoni G, Bracco A, Blasi N, Camera F, Grassi C, Million B, Paleni A, Pignanelli M, Vigezzi E, Wieland O, Matsuo M, Dossing T, Herskind B, Hagemann G B, Wilson J, Maj A, Kmiecik M, Lo Bianco G, Petrache C M, Castoldi M, Zucchiati A, De Angelis G, Napoli D, Bednarczyk P and Curien D 2005 Damping mechanisms and order-to-chaos transition in the warm rotating ${ }^{163}$ Er nucleus Phys. Rev. C72 034307

[100] Leoni S, Benzoni G, Blasi N, Bracco A, Brambilla S, Camera F, Corsi A, Crespi F C L, Mason P, Million B, Montanari D, Pignanelli M, Vigezzi E, Wieland O, Matsuo M, Shimizu Y R, Curien D, Duchene G, Robin J, Bednarczyk P, Castoldi M, Herskind B, Kmiecik M, Maj A, Meczynski W, Styczen J, Zieblinski M, Zuber K and Zucchiatti A 2008 Probing the order-to-chaos region in superdeformed ${ }^{151} \mathrm{~Tb}$ and ${ }^{196} \mathrm{~Pb}$ nuclei with continuum $\gamma$ transitions Phys. Rev. Lett. 101 142502

[101] Vandone V, Leoni S, Benzoni G, Blasi N, Bracco A, Brambilla S, Boiano C, Bottoni S, Camera F, Corsi A, Crespi F C L, Giaz A, Million B, Nicolini R, Pellegri L, Pullia A, Wieland O, Bortolato D, de Angelis G, Calore E, Gottardo A, Maron G, Napoli D R, Rosso D, Sahin E, Valiente-Dobon J J, Bazzacco D, Bellato M, Farnea E, Lunardi S, Menegazzo R, Mengoni D, Molini P, Michelagnoli C, Montanari D, Recchia F, Ur C A, Gadea A, Hüyük T, Cieplicka N, Maj A, Kmiecik M, Atac A, Akkoyun S, Kaskas A, Söderstrom P -A, Birkenbach B, Cederwall B, Coleman-Smith P J, Cullen D M, Désesquelles P, Eberth J, Görgen A, Grebosz J, Hess H, Judson D, Jungclaus A, Karkour N, Nolan P, Obertelli A, Reiter P, Salsac M D, Stezowski O, Theisen Ch, Matsuo M and Vigezzi E 2013 Global properties of K hindrance probed by the $\gamma$ decay of the warm rotating ${ }^{174} \mathrm{~W}$ nucleus Phys. Rev. C88 034312

[102] Walker P M 2005 High-K structures under extreme conditions Acta Phys. Pol. B36 1055-1061

[103] Walker P M 2006 Nuclear isomers: stepping stones to the unknown AIP Conf. Proc. 819 16-23 
[104] Uhrenholt H, Åberg S, Dobrowolski A, Døssing Th, Ichikawa T and Möller P 2013 Combinatorial nuclear level-density model Nucl. Phys. A 913 127-56

[105] Shi Y, Xu F R, Walker P M and Dracoulis G D 2012 Superdeformed multi-quasiparticle high-K states and possible isomers in $\mathrm{Pb}$ and $\mathrm{Po}$ isotopes Phys. Rev. C85 064304

[106] Åberg S 1995 High spins and exotic shapes Lect. Notes Phys. 441 211-29

[107] Kondev F G, Dracoulis G D, Byrne A P, Kibédi T and Bayer s 1997 Multi-quasiparticle states in ${ }^{179} \mathrm{Ta}$ and structural changes in the yrast line of the odd tantalum isotopes Nucl. Phys. A617 91-130

[108] Dracoulis G D 2000 High-K states as a probe of nuclear structure Phys. Scr. T88 54-61

[109] Stone N J, Stone J R, Walker P M and Bingham C R 2013 Quasi-particle and collective magnetism: Rotation, pairing and blocking in high-K isomers Phys. Lett. B726 675-9

[110] Muto S, Stone N J, Bingham C R, Stone J R, Walker P M, Audi G, Gaulard C, Koster U, Nikolov J, Nishimura K, Ohtsubo T, Podolyak Z, Risegari L, Simpson G S, Veskovic M and Walters W B 2014 Magnetic properties of ${ }^{177} \mathrm{Hf}$ and ${ }^{180} \mathrm{Hf}$ in the strong-coupling deformed model Phys. Rev. C89 044309

[111] Thakur P, Dogra R, Bhati A K, Bedi S C, Singh R P, Muralithar S and Bhowmik R K 2000 Measurement of the Electric Quadrupole Moment of the $9 / 2^{-}$and $21 / 2^{-}$Isomers in ${ }^{173} \mathrm{Ta}$ Hyperfine Interact. 131 103-109

[112] Broude C, Hass M, Goldring G, Alderson A, Ali I, Cullen D M, Fallon P, Hanna F, Roberts J W, Sharpey-Schafer J F 1991 The quadrupole moment of the K $=25$ isomer in ${ }^{182}$ Os Phys. Lett. B264 17-20

[113] Chowdhury P, Fabricius B, Christensen C, Azgui F, Bjørnholm S, Borggreen J, Holm A, Pedersen J, Sletten G, Bentley M A, Howe D, Mokhtar A R, Morrison J D, Sharpey-Schafer J F, Walker P M and Lieder R M 1988 Decay of high-spin isomers in Os nuclei by barrier penetration Nucl. Phys. A485 136-60

[114] Xu F R, Walker P M, Sheikh J A and Wyss R 1998 Multi-quasiparticle potential-energy surfaces Phys. Lett. B435 257-63

[115] Balabanski D L, Vyvey K, Neyens G, Coulier N, Coussement R, Georgiev G, Lepine-Szily A, Ternier S, Teughels S, Mineva M, Walker P M, Blaha P, Almehed D and Frauendorf S 2001 Static quadrupole moment of the five-quasiparticle $\mathrm{K}=35 / 2$ isomer in ${ }^{179} \mathrm{~W}$ studied with the level-mixing spectroscopy method Phys. Rev. Lett. 86 604-7

[116] Boos N, Le Blanc F, Krieg M, Pinard J, Huber G, Lunney M D, Le Du D, Meunier R, Hussonnois M, Constantinescu O, Kim J B, Briançon Ch, Crawford J E, Duong H T, Gangrski Y P, Kühl T, Markov B N, Oganessian Yu Ts, Quentin P, Roussiere B, Sauvage J 1994 Nuclear Properties of the Exotic High-Spin Isomer ${ }^{178 m}$ Hf from Collinear Laser Spectroscopy Phys. Rev. Lett. 72 2689-92

[117] Gangrsky Yu 2006 Laser spectroscopy of high spin isomers - a review Hyperfine Interact. 171 203-8

[118] Pillet N, Quentin P and Libert J 2002 Pairing correlations in an explicitly particle-number conserving approach Nucl. Phys. A697 141-63

[119] Bissell M L, Flanagan K T, Gardner M D, Avgoulea M, Billowes J, Campbell P, Cheal B, Eronen T, Forest D H, Huikari J, Jokinen A, Moore I D, Nieminen A, Penttilä H, Rinta-Antila S, Tordoff B, Tungate G and Äystö J 2007 On the decrease in charge radii of multi-quasi particle isomers Phys. Lett. B645 330-4

[120] Levins J M G, Benton D M, Billowes J, Campbell P, Cooper T G, Dendooven P, Evans D E, Forest D H, Grant I S, Griffith J A R, Huikari J, Jokinen A, Peräjärvi K, Tungate G, Yeandle G and Äystö J 1999 First On-Line Laser Spectroscopy of Radioisotopes of a Refractory Element Phys. Rev. Lett. 82 2476-2479

[121] Flanagan K T, Lynch K M, Billowes J, Bissell M L, Budincevic I, Cocolios T E, de Groote R P, De Schepper S, Fedosseev V N, Franchoo S, Garcia Ruiz R F, Heylen H, Marsh B A, Neyens G, Procter T J, Rossel R E, Rothe S, Strashnov I, Stroke H H and Wendt K D 
A 2013 Collinear Resonance Ionization Spectroscopy of Neutron-Deficient Francium Isotopes Phys. Rev. Lett. 111212501

[122] Cline D 1986 Nuclear shapes studied by Coulomb excitation Ann. Rev. Nucl. Part. Sci. 36 683716

[123] Sarriguren P, Rodriguez-Guzman R and Robledo L M 2008 Shape transitions in neutron-rich Yb, Hf, W, Os, and Pt isotopes within a Skyrme Hartree-Fock + BCS approach Phys. Rev. C 77 064322

[124] Robledo L M, Rodriguez-Guzman R and Sarriguren P 2009 Role of triaxiality in the ground-state shape of neutron-rich Yb, Hf, W, Os and Pt isotopes J. Phys. G36 115104

[125] Wheldon C, Garcés Narro J, Pearson C J, Regan P H, Podolyák Z, Warner D D, Fallon P, Macchiavelli A O and Cromaz M 2000 Yrast states in ${ }^{194}$ Os: The prolate-oblate transition region Phys. Rev. C63 011304

[126] Lane G J, Dracoulis G D, Kondev F G, Hughes R O, Watanabe H, Byrne A P, Carpenter M P, Chiara C J, Chowdhury P, Janssens R V F, Lauritsen T, Lister C J, McCutchan E, Seweryniak D, Stefanescu I and Zhu S 2010 Structure of the neutron-rich nuclei ${ }^{188} \mathrm{~W}$ and ${ }^{190} \mathrm{~W}$ and identification of a $10^{-}$isomer in ${ }^{190} \mathrm{~W}$ Phys. Rev. C82 051304(R)

[127] Podolyák Zs, Regan P H, Pfutzner M, Gerl J, Hellstrom M, Caamano M, Mayet P, Schlegel Ch, Aprahamian A, Benlliure J, Bruce A M, Butler P A, Cortina Gil D, Cullen D M, Doring J, Enqvist T, Rejmund F, Fox C, Garces Narro J, Geissel H, Gelletly W, Giovinazzo J, Gorska M, Grawe H, Grzywacz R, Kleinbohl A, Korten W, Lewitowicz M, Lucas R, Mach H, Mineva M, O'Leary C D, De Oliveira F, Pearson C J, Rejmund M, Sawicka M, Schaffner H, Schmidt K, Theisen Ch, Walker P M, Warner D D, Wheldon C, Wollersheim H J, Wooding S C and Xu F R 2000 Isomer Spectroscopy of Neutron Rich ${ }^{190} \mathrm{~W}_{116}$ Phys. Lett. B491 225-31

[128] Dracoulis G D, Lane G J, Byrne A P, Watanabe H, Hughes R O, Kondev F G, Carpenter M P, Janssens R V F, Lauritsen T, Lister C J, Seweryniak D, Zhu S, Chowdhury P, Shi Y and Xu F R 2014 Deep inelastic reactions and isomers in neutron-rich nuclei across the perimeter of the A $=180-190$ deformed region EPJ Web Conf. 6602033

[129] Dracoulis G D, Lane G J, Byrne A P, Hughes R O, N. Palalani, Kondev F G, Carpenter M P, Seweryniak D, Zhu S, Janssens R V F, Lister C J, Lauritsen T, Chowdhury P, Shi Y and Xu F R 2013 Isomers and excitation modes in the gamma-soft nucleus ${ }^{192}$ Os Phys. Lett. B720 330-5

[130] Dracoulis G D, Lane G J, Byrne A P, Watanabe H, Hughes R O, N. Palalani, Kondev F G, Carpenter M P, Seweryniak D, Zhu S, Janssens R V F, Lister C J, Lauritsen T, Chowdhury P, Shi Y and Xu F R 2012 Long-lived three-quasiparticle isomers in ${ }^{191} \mathrm{Ir}$ and ${ }^{193} \mathrm{Ir}$ with triaxial deformation Phys. Lett. B709 59-64

[131] Saitoh T R, Saitoh-Hashimoto N, Sletten G, Bark R A, Hagemann G B and Herskind B 2000 K-forbidden transition probabilities Phys. Scr. T88 67-71

[132] Caurier E, Martinez-Pinendo G, Nowacki F, Poves A and Zuker A P 2005 The shell model as a unified view of nuclear structure Rev. Mod. Phys. 77 427-88

[133] Maier K H and Rejmund M 2002 Shell model interaction between proton holes and between proton holes and neutron particles around ${ }^{208} \mathrm{~Pb}$ Eur. Phys. J. A14 349-53

[134] Brown B A 2000 Double-Octupole States in ${ }^{208} \mathrm{~Pb}$ Phys. Rev. Lett. 85 5300-3

[135] Coraggio L, Covello A, Gargano A, Itaco N and Kuo T T S 1999 Bonn potential and shell-model calculations for $\mathrm{N}=126$ isotones Phys. Rev. C60 064306

[136] Caurier E, Rejmund M and Grawe H 2003 Large-scale shell model calculations for the N=126 isotones Po-Pu Phys. Rev. C67 054310

[137] Andersson G, Larsson S E, Leander G, Möller P, Nilsson S G, Ragnarsson I, Åberg S, Bengtsson R, Dudek J, Nerlo-Pomorska B, Pomorski K and Szymanski Z 1976 Nuclear Shell Structure at Very High Angular Momentum Nucl. Phys. A268 205-56

[138] Andersson C G and Krumlinde J 1977 Oblate High Spin Isomers Nucl. Phys. A291 21-44

[139] Andersson C G, Hellstrom G, Leander G, Ragnarsson I, Åberg S, Krumlinde J, Nilsson S G and Szymanski Z 1978 Islands of High-Spin Yrast Isomers Nucl. Phys. A309 141-76 
[140] Døssing T, Neergård K, Matsuyanagi K and Hsi-Chen Chang 1977 Structure of Yrast traps Phys. Rev. Lett. 39 1395-7

[141] Matsuyanagi K, Døssing T and Neergård K 1978 High-Spin Isomers in Po, At and Rn in the Deformed Independent Particle Model Nucl. Phys. A307 253-76

[142] Butler P A and Nazarewicz W 1996 Intrinsic reflection asymmetry in atomic nuclei Rev. Mod. Phys. 68 349-421

[143] Dracoulis G D, Riess F, Baxter A M and Stuchbery A E 1991 Lifetimes in ${ }^{216}$ Ra and ${ }^{217} \mathrm{Ra}$; Development of Collectivity in Trans-Lead Nuclei J. Phys. G 17 1795-802

[144] Dracoulis G D, Riess F, Stuchbery A E, Bark R A, Gupta S L, Baxter A M and Kruse M 1989 Properties of $(15 / 2)^{-}$States in ${ }^{215} \mathrm{Ra}$ and ${ }^{217} \mathrm{Th}$; Evaluation of the $(15 / 2)^{-}$to $(9 / 2)^{+} \mathrm{E} 3$ strength in $\mathrm{N}=127$ isotones Nucl. Phys. A493 145-56

[145] Hauschild K, Rejmund M, Grawe H, Caurier E, Nowacki F, Becker F, Le Coz Y, Korten E, Döring J, Górska M, Schmidt K, Dorvaux O, Helariutta K, Jones P, Julin R, Juutinen S, Kettunen H, Leino M, Muikku M, Nieminen P, Rahkila P, Uusitalo J, Azaiez F and Belleguic M 2001 Isomer Spectroscopy in ${ }_{90}^{216} \mathrm{Th}_{126}$ and the Magicity of ${ }_{92}^{218} \mathrm{U}_{126}$ Phys. Rev. Lett. 87 072501

[146] Faessler A, Ploszajcak M and Devi K R S 1976 Possible mechanism for the formation of yrast traps at very high spins Phys. Rev. Lett. 36 1028-31

[147] Blomqvist J 1979 Structure of yrast states in the $N \sim 126$ and $N \sim 82$ regions from a shell model perspective Proceedings "Symposium on High-spin Phenomena", March 17, 1979, Argonne National Laboratory, Argonne, Illinois, ANL/PHY-79-4, p155-63

[148] Schiffer J P and True W W 1976 The effective interaction between nucleons deduced from nuclear spectra Rev. Mod. Phys. 48 191-217

[149] Molinari A, Johnson M B, Bethe H A and Alberico W M 1975 Effective Two-Body Interaction in Simple Nuclear Spectra Nucl. Phys. A239 45-73

[150] Kuo T T S and Herling G H 1971 NRL Report no. 2258 (Naval Research Laboratory, Washington, 1971), unpublished.

[151] Bayer S, Byrne A P, Dracoulis G D, Baxter A M, Kibédi T, Kondev F G, Mullins S M and McGoram T R 1999 High-Spin States, Yrast Isomers and Residual Interactions in the OddOdd Nucleus ${ }^{212}$ At Nucl. Phys. A650 3-36

[152] Szpak B, Maier K H, Smolkowska A S, Fornal B, Broda R, Carpenter M P, Cieplicka N, Janssens R V F, Krolas W, Pawlat T, Wrzesinski J and Zhu S 2011 Yrast structure of the two-protonand three-neutron-hole nucleus ${ }^{203} \mathrm{Hg}$ from the decay of a $53 / 2^{+}$isomer Phys. Rev. C83 064315

[153] Maier K H, Kibédi T, Dracoulis D G, Boutachkov P, Aprahamian A, Byrne A P, Davidson P M, Lane G J, Marie-Jeanne M, Nieminen P and Watanabe H 2007 Measurement of conversion electrons with the ${ }^{208} \mathrm{~Pb}(\mathrm{p}, \mathrm{n})^{208} \mathrm{Bi}$ reaction and derivation of the shell model proton neutron hole interaction from the properties of ${ }^{208}$ Bi Phys. Rev. C 76064304

[154] Maier K H and Rejmund M 2002 Shell Model Interaction between Proton Holes and between Proton Holes and Neutron Particles Around ${ }^{208} \mathrm{~Pb}$ Eur. Phys. J. A14 349-53

[155] Byrne A P and Dracoulis G D 1982 Energy Depression of High-Spin Core-Excited States in Radon Nuclei Nucl. Phys. A391 1-8

[156] Hübel H 2005 Magnetic rotation in nuclei Prog. Part. Nucl. Phys. 54 1-69

[157] Clark R M and Macchiavelli A O 2000 The Shears Mechanism in Nuclei Annu. Rev. Nucl. Part. Sci. 50 1-36

[158] Amita, Jain A K and Singh B 2000 Table of Magnetic Dipole Rotational Bands At. Data Nucl. Data Tables 74 283-331

[159] Dracoulis G D, Lane G J, Kibédi T and Nieminen P 2009 Characterization of the $32 \mu$ s isomer in ${ }^{189} \mathrm{~Pb}$ as a shears-mode bandhead Phys. Rev. C79 031302(R)

[160] Bergström I and Fant B 1985 Systematics of B(E3) Values in the Trans-Lead Region Phys. Scr. 31 26-30

[161] Poletti A R, Dracoulis G D, Byrne A P, Stuchbery A E, Poletti S J, Gerl J and Lewis P M 1985 Magnetic Moments, E3 Transitions and the Structure of High-Spin Core Excited States 
in ${ }^{211}$ Rn Phys.Lett. B 154 263-267

[162] Poletti S J, Dracoulis G D, Poletti A R, Byrne A P, Stuchbery A E and Gerl J 1986 gFactors in ${ }^{210} \mathrm{Rn}$ and Octupole Coupling of Core-Excited States in ${ }^{210} \mathrm{Rn},{ }^{211} \mathrm{Rn}$ and ${ }^{212} \mathrm{Rn}$ Nucl. Phys. A448 189-204

[163] Dracoulis G D, Davidson P M, Byrne A P, Fabricius B, Kibédi T, Baxter A M, Stuchbery A E, Poletti A R and Schiffer K J 1990 High-Spin Yrast Isomers in ${ }^{211} \mathrm{Rn}$ and ${ }^{212} \mathrm{Rn}$ with Enhanced E3 Decays Phys. Lett. B 246 31-35

[164] Byrne A P, Dracoulis G D, Fahlander C, Hübel H, Poletti A R, Stuchbery A E, Gerl J, Davie R F and Poletti S J 1986 Spectroscopy of High-Spin States in ${ }^{211,212,213}$ Fr Nucl. Phys. A448 $137-88$

[165] Poletti A R, Dracoulis G D, Byrne A P and Stuchbery A E 1987 The low-lying yrast structure of ${ }^{212}$ Po Nucl. Phys. A473 595-604

[166] Kudo H, Nomura T, Sueki K, Magara M and Yoshida N 1989 Isomeric transitions of ${ }^{211 m}$ Po and ${ }^{212 m} \mathrm{Po}$ and E4 effective charge Nucl. Phys. A494 203-13

[167] Dracoulis G D, Lane G J, Byrne A P, Davidson P M, Kibédi T, Nieminen P, Maier K H, Watanabe $\mathrm{H}$ and Wilson A N 2008 Neutron core excitations in the $\mathrm{N}=126$ nuclide ${ }^{210} \mathrm{Po}$ Phys. Rev. Cr7 034308

[168] McGoram T R, Dracoulis G D, Byrne A P, Poletti A R and Bayer S 1998 High-Spin Isomers in ${ }^{211} \mathrm{Po}$ and Related Structures in ${ }^{210} \mathrm{Po}$ and ${ }^{212} \mathrm{Po}$ Nucl. Phys. A637 469-90

[169] Lane G J, Byrne A P and Dracoulis G D 2003 High-Spin Isomers, Residual Interactions and Octupole Correlations in $\mathrm{N}=128$ Isotones ${ }^{211} \mathrm{Bi},{ }^{212} \mathrm{Po}$ ans ${ }^{213}$ At AIP Conf. Proc. 656 386-93

[170] Fornal B, Broda R, Królas W, Pawłat T, Wrzesiński J, Daly P J, Bhattacharyya P, Grabowski Z W, Zhang C T, Bazzacco D, Lunardi S, Rossi Alvarez C, de Angelis G and Napoli D R 1998 High Spin States Above the $\alpha$-Decaying Isomer In ${ }^{211}$ Po Eur. Phys. J. A1 355-7

[171] Lane G J, Broda R, Fornal B, Byrne A P, Dracoulis G D, Blomqvist J, Clark R M, Cromaz M, Deleplanque M A, Diamond R M, Fallon P, Janssens R V F, Lee I Y, Macchiavelli A O, Maier K H, Rejmund M, Stephens F S, Svensson C E, Vetter K, Ward D, Wiedenhover I and Wrzesiński $\mathrm{J} 2001$ Structure of Exotic Nuclei Near and Above ${ }^{208} \mathrm{~Pb}$ Populated via Deep-Inelastic Collisions Nucl. Phys. A682 71c-78c

[172] Garnsworthy A B, Thompson N J, Podolyák Zs, Walker P M, Williams S J, Dracoulis G D, de France G, Lane G J, Andgren K, Bruce A M, Byrne A P, Catford W N, Cederwall B, Jones G A, McGuirk B, Mandal S, Paul E S, Pucknell V, Redon N, Rosse B, Senior R J and Sletten G 2005 Spectroscopy of ${ }^{212} \mathrm{Po}$ and ${ }^{213} \mathrm{At}$ using a ${ }^{8} \mathrm{He}$ radioactive beam and EXOGAM J. Phys. G31 S1851-S1854

[173] Astier A, Porquet M -G, Delion D S and Schuck P 2010 Novel Manifestation of $\alpha$-Clustering Structures: New " $\alpha+{ }^{208} \mathrm{~Pb}$ " States in ${ }^{212} \mathrm{Po}$ Revealed by Their Enhanced E1 Decays Phys. Rev. Lett. 104042701

[174] Astier A, Porquet M -G, Delion D S and Schuck P 2010 Coexistence of " $\alpha+{ }^{208} \mathrm{~Pb}$ " cluster structures and single-particle excitations in ${ }_{84}^{212} \mathrm{Po}_{128}$ Eur. Phys. J. A46 165-85

[175] Astier A and Porquet M -G 2011 High-spin states in the five-valence-particle nucleus ${ }^{213}$ Po 2011 Phys. Rev. C83, 034302; Erratum Phys. Rev. C86 049905

[176] Astier A and Porquet M -G 2011 First observation of high-spin states in ${ }^{214}$ Po: Probing the valence space beyond ${ }^{208} \mathrm{~Pb}$ Phys. Rev. C83 034311

[177] Byrne A P, Lane G J and Dracoulis G D 2003 Residual interaction and high spin states in A=211 isobars Acta Phys. Pol. B34 2285-94

[178] Warburton E K 1991 Shell-model interpretation of the $\beta^{-}$decay of ${ }^{212} \mathrm{Bi}^{g},{ }^{212} \mathrm{Bi}^{m 1}$, and ${ }^{212} \mathrm{Bi}^{m 2}$ Phys. Rev. C44 261-7

[179] Chen L, Walker P M, Geissel H, Litvinov Yu A, Beckert K, Beller P, Bosch F, Boutin D, Caceres L, Carroll J J, Cullen D M, Cullen I J, Franzke B, Gerl J, Górska M, Jones G A, Kishada A, Knöbel R, Kozhuharov C, Kurcewicz J, Litvinov S A, Liu Z, Mandal S, Montes F, Münzenberg G, Nolden F, Ohtsubo T, Patyk Z, Plass W R, Podolyák Zs, Rigby S, Saito N, Saito T, 
Scheidenberger C, Simpson E S, Shindo M, Steck M, Sun B, Williams S J, Weick H, Winkler M, Wollersheim H -J and Yamaguchi T 2013 Direct Observation of Long-Lived Isomers in ${ }^{212} \mathrm{Bi}$ Phys. Rev. Lett. 110122502

[180] Andreyev A N, Huyse M, Van Duppen P, Weissman L, Ackermann D, Gerl J, Hessberger F P, Hofmann S, Kleinbohl A, Münzenberg G, Reshitko S, Schlegel C, Schaffner H, Cagarda P, Matos M, Saro S, Keenan A, Moore C, O'Leary C D, Page R D, Taylor M, Kettunen H, Leino M, Lavrentiev A, Wyss R and Heyde K 2000 A triplet of differently shaped spin-zero states in the atomic nucleus ${ }^{186} \mathrm{~Pb}$ Nature (London) $405430-433$

[181] Fossion R, Heyde K, Thiamova G and Van Isacker P 2003 Intruder bands and configuration mixing in lead isotopes Phys. Rev. C67 024306

[182] Ionescu-Bujor M, Iordachescu A, Ur C A, Marginean N, Suliman G, Bucurescu D, Brandolini F, Della Vedova F, Chmel S, Lenzi S M, Marginean R, Medina N H, Napoli D R, Pavan P and Ribas R V $2010 \mathrm{~g}$ factors of coexisting isomeric states in ${ }^{188} \mathrm{~Pb}$ Phys. Rev. C81 024323

[183] Jiao C F, Shi Y, Liu H L, Xu F R and Walker P M 2015 Shape-coexisting rotation in neutrondeficient $\mathrm{Hg}$ and $\mathrm{Pb}$ nuclei Phys. Rev. C91 034309

[184] Fornal B, Broda R, Maier K H, Wrzesiński J, Lane G J, Cromaz M, Macchiavelli A O, Clark R M, Vetter K, Byrne A P, Dracoulis G D, Carpenter M P, Janssens R V F, Wiedenhoever I, Rejmund M and Blomqvist J 2001 Effective Charge of the $\pi \mathrm{h}_{11 / 2}$ Orbital and the Electric Field Gradient of $\mathrm{Hg}$ from the Yrast Structure of ${ }^{206} \mathrm{Hg}$ Phys. Rev. Lett. 87212501

[185] Steer S J, Podolyák Zs, Pietri S, Górska M, Regan P H, Rudolph D, Werner-Malento E, Garnsworthy A B, Hoischen R, Gerl J, Wollersheim H J, Maier K H, Grawe H, Becker F, Bednarczyk P, Cáceres L, Doornenbal P, Geissel H, Grębosz J, Kelic A, Kojouharov I, Kurz N, Montes F, Prokopowicz W, Saito T, Schaffner H, Tashenov S, Heinz A, Pfützner M, KurtukianNieto T, Benzoni G, Jungclaus A, Balabanski D L, Brandau C, Brown B A, Bruce A M, Catford W N, Cullen I J, Dombrádi Zs, Estevez M E, Gelletly W, Ilie G, Jolie J, Jones G A, Kmiecik M, Kondev F G, Krücken R, Lalkovski S, Liu Z, Maj A, Myalski S, Schwertel S, Shizuma T, Walker P M and Wieland O 2008 Single-particle behavior at $\mathrm{N}=126$ : Isomeric decays in neutron-rich ${ }^{204} \mathrm{Pt}$ Phys. Rev. C78 061302(R)

[186] Wrzesiński J, Lane G J, Maier K H, Fornal B, Dracoulis G D, Rejmund M, Broda R, Byrne A P, Carpenter M P, Clark R M, Cromaz M, Janssens R V F, Machiavelli A O and Vetter K 2014 High-spin yrast isomers in ${ }^{204} \mathrm{Hg}$ Symposium "Nuclear Structure Physics with Advanced Gamma-Detector Arrays" (NSP13), June 10-12, 2013, Palazzo del Bo', Padova, Italy

[187] Fornal B, Szpak B, Janssens R V F, Broda R, Carpenter M P, Dracoulis G D, Maier K H, Lane G J, Wrzesiński J and Zhu S 2011 Neutron-particle and proton-hole excitations in the $\mathrm{N}=128$ isotones ${ }^{208} \mathrm{Hg}$ and ${ }^{209} \mathrm{Tl}$ from spectroscopy following ${ }^{208} \mathrm{~Pb}+{ }^{238} \mathrm{U}$ deep-inelastic reactions J. Phys. Conf. Ser. 267012035

[188] Broda R, Maier K H, Fornal B, Wrzesiński J, Szpak B, Carpenter M P, Janssens R V F, Krolas W, Pawlat T and Zhu S 2011 High-spin states and isomers in the one-proton-hole and threeneutron-hole ${ }^{204} \mathrm{Tl}$ isotope Phys. Rev. C84 014330

[189] Wrzesiński J, Broda R, Fornal B, Królas W, Pawłat T, Carpenter M P, Janssens R V F, Seweryniak D, Lunardi S, Ur C A, Viesti G, Cinausero M, Marginean N and Maier K H 2004 The $\pi \mathrm{h}_{11 / 2}^{1} \nu \mathrm{i}_{13 / 2}^{-2}$ three-hole isomeric state and octupole core excitation in the ${ }^{205} \mathrm{Tl}$ nucleus Eur. Phys. J. A20 57-8

[190] Lane G J, Maier K H, Byrne A P, Dracoulis G D, Broda R, Fornal B, Carpenter M P, Clark R M, Cromaz M, Janssens R V F, Macchiavelli A O, Wiedenhover I and Vetter K 2005 High-spin isomers and three-neutron valence configurations in ${ }^{211} \mathrm{~Pb}$ Phys. Lett. B606 34-42

[191] Cieplicka N, Maier K H, Fornal B, Szpak B, Janssens R V F, Alcorta M, Broda R, Carpenter M P, Chiara C J, Hoffman C R, Kay B P, Kondev F G, Królas W, Lauritsen T, Lister C J, McCutchan E A, Pawłat T, Rogers A M, Seweryniak D, Sharp N, Walters W B, Wrzesiński $\mathrm{J}$ and Zhu S 2012 Yrast structure of ${ }^{206} \mathrm{Bi}$ : Isomeric states and one-proton-particle, threeneutron-hole excitations Phys. Rev. C86 041304 
[192] Pfützner M, Armbruster P, Baumann T, Benlliure J, Bernas M, Catford W N, Cortina-Gil D, Daugas J M, Geissel H, Górska M, Grawe H, Grzywacz R, Hellström M, Iwasa N, Janas Z, Junghans A R, Karny M, Leenhardt S, Lewitowicz M, Mueller A C, de Oliviera F, Regan P H, Rejmund M, Rykaczewski K and Sümmerer K 1998 New isotopes and isomers produced by the fragmentation of $238 \mathrm{U}$ at $1000 \mathrm{MeV} /$ nucleon Phys. Lett. B444 32-7

[193] Gottardo A, Valiente-Dobon J J, Benzoni G, Nicolini R, Gadea A, Lunardi S, Boutachkov P, Bruce A M, Górska M, Grebosz J, Pietri S, Podolyák Zs, Pfützner M, Regan P H, Weick H, Alcántara Núñez J, Algora A, Al-Dahan N, de Angelis G, Ayyad Y, Alkhomashi N, Allegro P R P, Bazzacco D, Benlliure J, Bowry M, Bracco A, Bunce M, Camera F, Casarejos E, Cortes M L, Crespi F C L, Corsi A, Bacelar A M D, Deo A Y, Domingo-Pardo C, Doncel M, Dombradi Zs, Engert T, Eppinger K, Farrelly G F, Farinon F, Farnea E, Geissel H, Gerl J, Goel N, Gregor E, Habermann T, Hoischen R, Janik R, Klupp S, Kojouharov I, Kurz N, Lenzi S M, Leoni S, Mandal S, Menegazzo R, Mengoni D, Million B, Morales A I, Napoli D R, Naqvi F, Nociforo C, Prochazka A, Prokopowicz W, Recchia F, Ribas R V, Reed M W, Rudolph D, Sahin E, Schaffner H, Sharma A, Sitar B, Siwal D, Steiger K, Strmen P, Swan T P D, Szarka I, Ur C A, Walker P M, Wieland O, Wollersheim H -J, Nowacki F, Maglione E and Zuker A P 2012 New Isomers in the Full Seniority Scheme of Neutron-Rich Lead Isotopes: The Role of Effective Three-Body Forces Phys. Rev. Lett. 109 162502

[194] Gottardo A, Valiente-Dobon J J, Benzoni G, Gadea A, Lunardi S, Boutachkov P, Bruce A M, Górska M, Grebosz J, Pietri S, Podolyák Zs, Pfützner M, Regan P H, Weick H, Alcántara Núñez J, Algora A, Al-Dahan N, de Angelis G, Ayyad Y, Alkhomashi N, Allegro P R P, Bazzacco D, Benlliure J, Bowry M, Bracco A, Bunce M, Camera F, Casarejos E, Cortes M L, Crespi F C L, Corsi A, Bacelar A M D, Deo A Y, Domingo-Pardo C, Doncel M, Dombradi Zs, Engert T, Eppinger K, Farrelly G F, Farinon F, Farnea E, Geissel H, Gerl J, Goel N, Gregor E, Habermann T, Hoischen R, Janik R, John P R, Klupp S, Kojouharov I, Kurz N, Lenzi S M, Leoni S, Mandal S, Menegazzo R, Mengoni D, Million B, Modamio V, Morales A I, Napoli D R, Naqvi F, Nicolini R, Nociforo C, Prochazka A, Prokopowicz W, Recchia F, Ribas R V, Reed M W, Rudolph D, Sahin E, Schaffner H, Sharma A, Sitar B, Siwal D, Steiger K, Strmen P, Swan T P D, Szarka I, Ur C A, Walker P M, Wieland O and Wollersheim H -J 2013 New $\mu \mathrm{s}$ isomers in the neutron-rich ${ }^{210} \mathrm{Hg}$ nucleus Phys. Lett. B725 292-6

[195] Dracoulis G D, Lane G J, Byrne A P, Davidson P M, Kibédi T, Nieminen P H, Watanabe H and Wilson A N 2008 High-spin isomers in ${ }^{212} \mathrm{Rn}$ in the region of triple neutron core-excitations Phys. Lett. B662 19-25

[196] Dracoulis G D, Lane G J, Byrne A P, Davidson P M, Kibédi T, Nieminen P H, Watanabe H, Wilson A N, Liu H L and Xu F R 2009 Structure of the $\mathrm{N}=126$ nuclide ${ }^{212} \mathrm{Rn}$ : Valence and core excited configurations Phys. Rev. C80 054320

[197] Horn D, Häusser O, Faestermann T, McDonald A B, Alexander T K, Beene J R and Herrlander C J 1977 Core-Excited High-Spin Isomers in ${ }^{212}$ Rn Phys. Rev. Lett. 39 389-91

[198] Horn D, Häusser O, Haas B, Alexander T K, Faestermann T, Andrews H R and Ward D 1979 High Spin Yrast States in N=126 Isotones Nucl. Phys. A317 520-34

[199] Stuchbery A E, Dracoulis G D, Byrne A P and Poletti A R 1988 Spectroscopy of ${ }^{212} \mathrm{Rn}$ Nucl. Phys. A486 397-413

[200] Dracoulis G D 1990 Structure of high-spin isomers in trans-lead nuclei Proceedings of the $X X V$ Zakopane School on Physics, Zakopane, Poland 28 April- 12 May 1990: Selected Topics in Nuclear Structure Ed. Jan Styczen and Z. Stachura, Vol. 2 p3-28, World Scientific, Singapore

[201] Bayer S, Byrne A P, Dracoulis G D, Baxter A M, Kibédi T and Kondev F G 2001 Core-Excited States and Core-Polarization Effects in ${ }^{210}$ At and ${ }^{211}$ At Nucl. Phys. A694 3-62

[202] Byrne A P, Bayer S, Dracoulis G D, Kibédi T 1998 Blocking of Octupole Correlations Deduced from the Decay of a Multiparticle Isomer in ${ }^{212}$ At Phys. Rev. Lett. 80 2077-80

[203] Dafni E, Hass M, Naim E, Rafailovich M H, Berger A, Grawe H and Mahnke H -E 1985 Electric Quadrupole Moment of the ${ }^{211} \mathrm{Rn} 63 / 2^{-}$Isomer: Absence of core deformation at very high 
spins Phys. Rev. Lett. 55 1269-72

[204] Poletti A R, Byrne A P, Dracoulis G D, Kibédi T and Davidson P M 2005 High spin states in ${ }^{210} \mathrm{Rn}$ approaching the region of 3-particle-hole neutron excitations Nucl. Phys. A756 83-117

[205] Byrne A P, Müsseler R, Hübel H, Maier K H and Kluge H 1990 Quadrupole Moments of Isomeric States in ${ }^{213} \mathrm{Fr}$ and ${ }^{212} \mathrm{Fr}$ Nucl. Phys. A516 145-56

[206] Hardeman F, Neyens G, Scheveneels G, Nouwen R, S'heeren G, Van Den Bergh M, Coussement R, Byrne A P, Müsseler R, Hübel H and Baldsiefen G 1991 Quadrupole Moments of High Spin Isomers in ${ }^{213} \mathrm{Fr},{ }^{212} \mathrm{Fr}$, and ${ }^{211} \mathrm{Fr}$ Phys. Rev. C43 514-520

[207] Dracoulis G D, Davidson P M, Lane G J, Byrne A P, Kibédi T, Nieminen P H, Watanabe H and Wilson A N 2009 Spectroscopy and high-spin structure of ${ }^{209}$ Fr Phys. Rev. C79 054313

[208] Nilsson S G, Tsang C F, Sobiczewski A, Szymański Z, Wycech S, Gustafson C, Lamm I-L, Möller P and Nilsson B 1969 On the Nuclear Structure and Stability of Heavy and Superheavy Elements Nucl. Phys. A131 1-66

[209] Lönnroth T, Beausang C W, Fossan D B, Hildingsson L, Piel Jr W F and Warburton E K 1989 Very-High-Spin and Core-Coupled States in ${ }^{213} \mathrm{Rn}$. A Test of the Applicability of the Deformed Independent Particle Model Phys. Scr. 39 56-65

[210] Nazarewicz W, Dudek J, Bengtsson R, Bengtsson T and Ragnarsson I 1985 Microscopic study of the high-spin behaviour in selected $\mathrm{A} \approx 80$ nuclei Nucl. Phys. A435 397-447

[211] Dracoulis G D, Fahlander C and Poletti A R 1981 High-Spin Isomer in ${ }^{211} \mathrm{Rn}$, and the Shape of the Yrast Line Phys. Rev. C24 2386-9

[212] Sagawa H and Arima A 1988 Static and Dynamic Quadrupole Moments of High-Spin Isomers in the Pb-Region Phys. Lett. B202 15-20

[213] Døssing, Neergård K, and Sagawa H 1981 Yrast Spectra in the ${ }^{146}$ Gd Region Phys. Scr. 24 258-65

[214] Xu F R, Wyss R and Walker P M 1999 Mean-field and blocking effects on odd-even mass differences and rotational motion of nuclei Phys. Rev. C60 051301(R)

[215] Odahara A, Gono Y, Fukuchi T, Wakabayashi Y, Sagawa H, Satuła W and Nazarewicz W 2005 Pairing correlations in high-spin isomers Phys. Rev. C72 061303(R)

[216] McNeill J H, Chishti A A, Daly P J, Gelletly W, Hotchkis M A C, Piiparinen M Varley B J, Woods P J and Blomqvist J 1993 Isomeric Decay Studies using a Recoil Mass Separator. Exotic $\mathrm{N}=82$ and 83 Nuclei ${ }^{153} \mathrm{Yb},{ }^{153} \mathrm{Lu},{ }^{154} \mathrm{Lu}$ and ${ }^{154} \mathrm{Hf}$ Z. Phys. A344 369-79

[217] McNeill J H, Chishti A A, Daly P J, Hotchkis M A C, Piiparinen M and Varley B J 1989 A 15 $\mu$ s Isomer in the $\mathrm{Z}=70 \mathrm{~N}=83$ Nucleus ${ }^{153} \mathrm{Yb}$ Z. Phys. A332 105-6

[218] McNeill J H 1987 The proton-rich $\mathrm{N}=82$ and $\mathrm{N}=83$ nuclei ${ }^{151} \mathrm{Tm},{ }^{150} \mathrm{Ho},{ }^{151} \mathrm{Er}$ and ${ }^{152} \mathrm{Tm}$ Diss. Abst. Int. 474517

[219] McNeill J, Broda R, Chung Y H, Daly P J, Grabowski Z W, Helppi H, Kortelahti M, Janssens R V F, Khoo T L, Lawson R D, Radford D C and Blomqvist J 1986 Level Structure of Proton-Rich $\mathrm{N}=83$ Nuclei ${ }^{150} \mathrm{Ho}$ and ${ }^{152} \mathrm{Tm}$ Z. Phys. A325 27 -35

[220] Fukuchi T, Hori T, Masue T, Tajiri K, Sato A, Furukawa T, Odahara A, Shimoda T, Wakabayashi Y, Gono Y, Suzuki T, Ukai M, Wakui T, Yamazaki A, Miyashita Y, Sato N, Tateoka M, Ohguma M, Shinozuka T, Koike T, Shirotori K, Miura Y, Kinoshita S, Ma Y, Fu Y Y and Tamura H 2009 Revised spin-parity assignment and a new interpretation of the high-spin isomer in ${ }^{151} \mathrm{Er}$ Eur. Phys. J. A39 49-51

[221] André S, Foin C, Barci V, Barnéoud D, Genevey J and Gizon A 1990 High-Spin Yrast Isomer in the $\mathrm{N}=83{ }^{151} \mathrm{Er}$ Nucleus Z. Phys. A337 349-50

[222] Foin C, Gizon A, Barnéoud D, Genevey J, Gizon J, Santos D, Farget F, Paris P, Liang C F, Bucurescu D and Plochocki A 2000 New Investigation of the Decay of the High-Spin Isomer in ${ }^{151}$ Er Eur. Phys. J. A8 451-4

[223] Collatz R, Kleinheinz P, Zhang C T, Keller H, Kirchner R, Klepper O and Roeckl E 1997 GammaDecay of the $(27 / 2)^{-}$0.6s E3-Isomer in ${ }_{68}^{151} \mathrm{Er}_{83}$ Z. Phys. A359 115-6

[224] Barden R, Plochocki A, Schardt D, Rubio B, Ogawa M, Kleinheinz P, Kirchner R, Klepper O 
and Blomqvist J 1988 Beta-Decay of 27/2- Isomers in $\mathrm{N}=83$ Nuclei Z. Phys. A329 11-20

[225] Fukuchi T, Tanaka S, Sasaki T, Gono Y, Odahara A, Morikawa T, Shibata M, Watanabe H, Motomura S, Tsutsumi T, Kashiyama O, Saitoh K, Wakabayashi Y, Kishida T, Kubono S and Ishihara M 2006 Level structure and excitation energy of a high-spin isomer in ${ }^{150} \mathrm{Ho}$ Phys. Rev. C73067303

[226] Gono Y, Odahara A, Fukuchi T, Ideguchi E, Kishida T, Kubo T, Watanabe H, Motomura S, Saito K, Kashiyama O, Morikawa T, Cederwall B, Zhang Y H, Zhou X H, Ishihara M and Sagawa H 2002 Systematics of high-spin isomers in $\mathrm{N}=83$ isotones and a high-spin isomer beam Eur. Phys. J. A13 5-8

[227] Watanabe H, Ueno H, Kameda D, Sato W, Yoshimi A, Miyoshi H, Kishida T, Kobayashi Y, Odahara A, Gono Y and Asahi K 2003 g-Factor of the high-spin isomer in ${ }^{149}$ Dy and a multiquasiparticle configuration caused by the $\mathrm{N}=82$ core excitations Nucl. Phys. A728 365-78

[228] Ideguchi E, Gono Y, Mitarai S, Morikawa T, Odahara A, Kidera M, Sibata M, Tsuchida H, Miyazaki K, Oshima M, Hatsukawa Y, Hamada S, Iimura H, Shibata M, Ishii T, Murakami T, Kusakari H, Sugawara M, Kishida T, Morita K, Kumagai H and Ishihara M 1995 High-Spin States in ${ }^{148} \mathrm{~Tb}$ Z. Phys. A352 363-4

[229] Broda R, Kleinheinz P, Lunardi S, Styczen J and Blomqvist J 1982 The Decay of the $560 \mathrm{~ns}$ Isomer at $8.6 \mathrm{MeV}$ in ${ }_{64}^{147} \mathrm{Gd}_{83}$ Z. Phys. A305 281-8

[230] Borggreen J, Sletten G, Bjørnholm S, Pedersen J, Del Zoppo A, Radford D C, Janssens R V F, Chowdhury P, Emling H, Frekers D and Khoo T L 1987 Single Particle Radiation between High Spin States in ${ }^{147}$ Gd Nucl. Phys. A466 371-84

[231] Ideguchi E, Zhou X H, Gono Y, Mitarai S, Morikawa T, Kidera M, Tsuchida H, Shibata M, Watanabe H, Miyake M, Odahara A, Oshima M, Hatsukawa Y, Hamada S, Iimura H, Shibata M, Ishii T, Kishida T and Ishihara M 1999 A New High Spin Isomer in ${ }^{146}$ Eu Eur. Phys. J. A6 $387-90$

[232] Ercan A, Broda R, Kleinheinz P, Piiparinen M, Julin R and Blomqvist J 1988 The Two Particle-Phonon Multiplet $\left(\pi \mathrm{h}_{11 / 2} \nu \mathrm{f}_{7 / 2}\right) \otimes 3^{-}$and Higher-Lying Four-Nucleon Yrast States in the Doubly-Odd Nucleus ${ }_{63}^{146} \mathrm{Eu}_{83}$ Z. Phys. A329 63-8

[233] Odahara A, Gono Y, Mitarai S, Ideguchi E, Kidera M, Shibata M, Morikawa T, Kishida T, Morita K, Yoshida A, Kumagai H, Zhang Y H, Ferragut A, Murakami T, Oshima M, Iimura H, Shibata M, Hamada S, Kusakari H, Sugawara M, Ogawa M, Nakajima M, Min B J, Kim J C, Chae S J and Sagawa H 1994 High-Spin States of ${ }^{145} \mathrm{Sm}$ Z. Phys. A350 185-6

[234] Odahara A, Gono Y, Mitarai S, Morikawa T, Shizuma T, Kidera M, Shibata M, Kishida T, Ideguchi E, Morita K, Yoshida A, Kumagai H, Zhang Y H, Ferragut A, Murakami T, Oshima M, Iimura H, Shibata M, Hamada S, Kusakari H, Sugawara M, Ogawa M, Nakajima M, Min B J, Kim J C, Chae S J and Sagawa H 1997 High-Spin Isomer and Level Structure of ${ }^{145} \mathrm{Sm}$ Nucl. Phys. A620 363-84

[235] Jessen K, Andrejtscheff W, Bergstrom M, von Brentano P, Dewald A, Herskind B, Meise H, Schumacher C, Sletten G, Stuch O, Weisshaar D, Wiedenhover I and Wrzesinski J 1999 A New High-Spin Isomer in ${ }^{145} \mathrm{Sm}$ Eur. Phys. J. A4 9-10

[236] El-Badry A M, Kuroyanagi T, Mitarai S, Odahara A, Gono Y, Morinobu S and Ogawa K 1998 Recoil Distance Half-Life Measurements of the Excited States in ${ }^{145} \mathrm{Sm}$ Eur. Phys. J. A3 133-8

[237] Ferragut A, Gono Y, Murakami T, Morikawa T, Zhang Y H, Morita K, Yoshida A, Oshima M, Kusakari H, Sugawara M, Ogawa M, Nakajima M, Mitarai S, Odahara A, Ideguchi E, Shizuma T, Kidera M, Kim J C, Chae S -J, Min B -J and Kumagai H 1993 A New High-Spin Isomer in ${ }^{145} \mathrm{Sm}$ J. Phys. Soc. Jpn. 62 3343-4

[238] Murakami T, Gono Y, Ferragut A, Zhang Y H, Morita K, Yoshida A, Ogawa M, Nakajima M, Min B J, Kumagai H, Oshima M, Morikawa T, Sugawara M and Kusakari H 1993 High-Spin Isomeric State in ${ }^{144} \mathrm{Pm}$ Z. Phys. A345 123-4

[239] Zhou X H, Tsuchida H, Gono Y, Odahara A, Ideguchi E, Morikawa T, Shibata M, Watanabe H, Miyake M, Tsutsumi T, Motomura S, Kishida T, Mitarai S and Ishihara M 1999 Level 
structure in ${ }^{143} \mathrm{Nd}$ Phys. Rev. C61 014303

[240] Häusser O, Taras P, Trautmann W, Ward D, Alexander T K, Andrews H R, Haas B and Horn D 1979 g Factors of High-Spin Yrast Traps in ${ }^{146,147}$ Gd Phys. Rev. Lett. 42 1451-4

[241] Dafni E, Bendahan J, Broude C, Goldring G, Hass M, Naim E, Rafailovich M H, Chasman C, Kistner O C and Vajda S 1985 Nuclear Polarization and Signs of Quadrupole Moments of High-Spin Gd Isomers Nucl. Phys. A443 135-56

[242] Häusser O, Mahnke H E, Alexander T K, Andrews H R, Sharpey-Schafer J F, Swanson M L, Ward D, Taras P and Keinonen J 1982 Quadrupole Moments of Yrast Isomers in ${ }^{144,147,148} \mathrm{Gd}$ Nucl. Phys. A379 287-312

[243] Odahara A, Gono Y, Fukuchi T, Wakabayashi Y, Sagawa H and Yoshida N 2008 Possible Shape isomers at High-Spin States of N=83 Isotones J. Phys. Soc. Jpn. 77114201

[244] Janssens R V F and Khoo T L 1991 Superdeformed Nuclei Annu. Rev. Nucl. Part. Sci. 41 321-55

[245] Takahara S, Tajima N and Onishi N, Study of Superdeformation in Non-Rotating States using the Skyrme-Hartree-Fock Method Nucl. Phys. A642 461-79

[246] Girod M, Delaroche J P and Berger J F 1988 Preliminary Selection of Candidates for Shape Isomerism from Even-Even Nuclei with Masses $\mathrm{A}<208$ Phys. Rev. C38 1519-22

[247] Bonche P, Krieger S J, Quentin P, Weiss M S, Meyer J, Meyer M, Redon N, Flocard H and Heenen P -H 1989 Superdeformation and Shape Isomerism at Zero Spin Nucl. Phys. A500 308-22

[248] Krieger S J, Bonche P, Weiss M S, Meyer J, Meyer M, Flocard H and Heenen P -H 1992 SuperDeformation and Shape Isomerism: Mapping the isthmus Nucl. Phys. A542 43-52

[249] Bonche P, Dobaczewski J, Flocard H, Heenen P -H and Meyer J 1990 Analysis of the Generator Coordinate Method in a Study of Shape Isomerism in ${ }^{194} \mathrm{Hg}$ Nucl. Phys. A510 466-502

[250] Bonche P, Dobaczewski J, Flocard H, Heenen P -H, Krieger S J, Meyer J and Weiss M S 1990 Quadrupole Collective Correlations and the Depopulation of the Superdeformed Bands in Mercury Nucl. Phys. A519 509-20

[251] Tajima T, Flocard H, Bonche P, Dobaczewski J and Heenen P -H, 1992 Generator coordinate kernels between zero- and two-quasiparticle BCS states Nucl. Phys. A542 355-67

[252] Krieger S J, Bonche P, Flocard H, Heenen P -H, Weiss M S 1994 GCM Calculation of the E2 Decay Lifetimes of Shape Isomers Nucl. Phys. A572 384-400

[253] Krieger S J, Bonche P, Flocard H, Heenen P -H , Mehrem R and Weiss M S 1996 Generator Coordinate Method Calculation of the E2 Decay Lifetime of Shape Isomers in the Actinide Region Phys. Rev. C54 2399-403

[254] Delaroche J -P, Girod M, Goutte H and Libert J 2006 Structure properties of even-even actindies at normal and super deformed shapes analysed using the Gogny force Nucl. Phys. A771 103-68

[255] Bürvenich T, Bender M, Maruhn J A and Reinhard P-G 2004 Systematics of fission barriers in superheavy elements Phys. Rev. C69 014307

[256] Jachimowicz P, Kowal M and Skalski J 2011 Superdeformed oblate superheavy nuclei Phys. Rev. C83054302

[257] Liu Hong-Liang 2013 Oblate Superdeformed High-K States in Superheavy Nuclei Commun. Theor. Phys. 60 577-80

[258] Minkov N and Walker P M 2012 Magnetic moments of K isomers as indicators of octupole collectivity Eur. Phys. J. A48 80

[259] Liu H L and Xu F R 2013 Enhanced octupole correlation due to unpaired nucleons in actinide K-isomeric states Phys. Rev. C87 067304

[260] Möller P, Sierk A J, Bengtsson R, Sagawa H and Ichikawa T 2009 Global Calculation of Nuclear Shape Isomers Phys. Rev. Lett. 103212501

[261] Möller P, Sierk A J, Bengtsson R, Sagawa H and Ichikawa T 2012 Nuclear Shape Isomers At. Data Nucl. Data Tables 98 149-300

[262] Marinov A, Gelberg S and Kolb D 2001 Discovery of Long-Lived Shape Isomeric States which Decay by Strongly Retarded High-Energy Particle Radioactivity Int. J. Mod. Phys. E10 185- 
208

[263] Marinov A, Rodushkin I, Kashiv Y, Halicz L, Segal I, Pape A, Gentry R V, Miller H W, Kolb D and Brandt R 2007 Existence of long-lived isomeric states in naturally-occurring neutrondeficient Th isotopes Phys. Rev. C76 021303(R)

[264] Marinov A, Gelberg S, Kolb D, Brandt R and Pape A 2003 Coherent Description for Hitherto Unexplained Radioactivities by Super- and Hyperdeformed Isomeric States Int. J. Mod. Phys. E12 661-8

[265] Barber R C and De Laeter J R 2009 Comment on "Existence of long-lived isomeric states in naturally-occurring neutron-deficient Th isotopes" Phys. Rev. C79 049801

[266] Marinov A, Rodushkin I, Kashiv Y, Halicz L, Segal I, Pape A, Gentry R V, Miller H W, Kolb D and Brandt R 2009 Reply to "Comment on 'Existence of long-lived isomeric states in naturallyoccurring neutron-deficient Th isotopes" Phys. Rev. C79 049802

[267] Polikanov S M, Druin V A, Karnaukhov V A, Mikheev V L, Pleve A A, Skobelev N K, TerAkopyan G M and Fomichev V A 1962 Spontaneous Fission with an Anomalously Short Period Zhur. Eksptl. i Teoret. Fiz. 42 1464-71; Soviet Phys. JETP 15 1016-21

[268] Britt H C 1973 Properties of fission isomers At. Data Nucl. Data Tables 12 407-14

[269] Vandenbosch R 1977 Spontaneously Fissioning Isomers Ann. Rev. Nucl. Sci. 27 1-35

[270] Bjørnholm S and Lynn J E 1980 The Double-Humped Fission Barrier Rev. Mod. Phys. 52 725-931

[271] Metag V, Habs D and Specht H J 1980 Spectroscopic Properties of Fission Isomers Phys. Rep. 65 $1-41$

[272] Hall H L and Hoffman D C 1992 Delayed Fission Annu. Rev. Nucl. Part. Sci. 42 147-75

[273] Singh B, Zywina R and Firestone R B 2002 Table of Superdeformed Nuclear Bands and Fission Isomers Nucl. Data Sheets 97 241-592

[274] Thirolf P G and Habs D 2002 Spectroscopy in the Second and Third Minimum of Actinide Nuclei Prog. Part. Nucl. Phys. 49 325-402

[275] Liu H L, Xu F R, Sun Y, Walker P M and Wyss R 2011 On the stability of high-K isomers in the second well of actinide nuclei Eur. Phys. J. A47 135

[276] Ren Z and Xu C 2005 Spontaneous fission half-lives of heavy nuclei in ground state and in isomeric state Nucl. Phys. A759 64-78

[277] Möller P, Sierk A J, Ichikawa T, Iwamoto A, Bengtsson R, Uhrenholt H and Åberg S 2009 Heavy-element fission barriers Phys. Rev. C79 064304

[278] Abusara H, Afanasjev A V and Ring P 2010 Fission barriers in actinides in covariant density functional theory: The role of triaxiality Phys. Rev. C82 044303

[279] Åberg S, Flocard H and Nazarewicz W 1990 Nuclear Shapes in Mean Field Theory Annu. Rev. Nucl. Part. Sci. $40439-528$

[280] Dancer H, Bonche P, Flocard H, Heenen P-H, Meyer J and Meyer M 1998 Monopole strength and decay out of superdeformed bands in the $\mathrm{A}=190$ mass region from theories beyond the mean field Phys. Rev. C58 2068-2072

[281] Zganjar E F and Wood J L 1990 Electric Monopole transitions and shape coexistence in nuclei Nucl. Phys. A520 427c-436c

[282] A.Ghiorso, K.Eskola, P.Eskola, M.Nurmia 1973 Isomeric States in ${ }^{250}$ Fm and ${ }^{254}$ No Phys. Rev. Cy 2032-6

[283] Leino M and Hessberger F P 2004 The Nuclear Structure of Heavy-Actinide and Transactinide Nuclei Ann. Rev. Nucl. Part. Sci. 54 175-215

[284] Herzberg R -D 2004 Spectroscopy of Superheavy Elements J. Phys. G 30 R123-41

[285] Herzberg R -D and Greenlees P 2008 In-beam and Decay Spectroscopy of Trans-Fermium Nuclei Prog. Part. Nucl. Phys. 61 674-720

[286] Herzberg R -D and Cox D M 2011 Spectroscopy of Actinide and Transactinide Nuclei Radiochim. Acta 99 441-57

[287] Clark R 2011 The Heaviest Atomic Nuclei and Their Metastable States Nucl. Phys. News 21 No. 3, 13-6 
[288] Greenlees P T 2013 Understanding the Nuclear Structure of Heavy Elements Phys. Scr. T152 014016

[289] Herzberg R -D, Greenlees P T, Butler P A, Jones G D, Venhart M, Darby I G, Eeckhaudt S, Eskola K, Grahn T, Gray-Jones C, Hessberger F P, Jones P, Julin R, Juutinen S, Ketelhut S, Korten W, Leino M, Leppänen A -P, Moon S, Nyman M, Page R D, Pakarinen J, Pritchard A, Rahkila P, Sarén J, Scholey C, Steer A, Sun Y, Theisen Ch and Uusitalo J 2006 Nuclear Isomers in Superheavy Elements as Stepping Stones Towards the Island of Stability Nature 442 896-9

[290] Tandel S K, Khoo T L, Seweryniak D, Mukherjee G, Ahmad I, Back B, Blinstrup R, Carpenter M P, Chapman J, Chowdhury P, Davids C N, Hecht A A, Heinz A, Ikin P, Janssens R V F, Kondev F G, Lauritsen T, Lister C J, Moore E F, Peterson D, Reiter P, Tandel U S, Wang X and Zhu S 2006 K Isomers in ${ }^{254}$ No: Probing Single-Particle Energies and Pairing Strengths in the Heaviest Nuclei Phys. Rev. Lett. 97082502

[291] Hessberger F P, Antalic S, Sulignano B, Ackermann D, Heinz S, Hofmann S, Kindler B, Khuyagbaatar J, Kojouharov I, Kuusiniemi P, Leino M, Lommel B, Mann R, Nishio K, Popeko A G, Šáro Š, Streicher B, Uusitalo J, Venhart M and Yeremin A V 2010 Decay Studies of K Isomers in ${ }^{254}$ No Eur. Phys. J. A43 55-66

[292] Greenlees P T, Herzberg R -D, Ketelhut S, Butler P A, Chowdhury P, Grahn T, Gray-Jones C, Jones G D, Jones P, Julin R, Juutinen S, Khoo T -L, Leino M, Moon S, Nyman M, Pakarinen J, Rahkila P, Rostron D, Sarén J, Scholey C, Sorri J, Tandel S K, Uusitalo J and Venhart M 2008 High-K structure in ${ }^{250} \mathrm{Fm}$ and the deformed shell gaps at $\mathrm{N}=152$ and $\mathrm{Z}=100$ Phys. Rev. C78 $021303(R)$

[293] Sulignano B, Theisen Ch, Delaroche J -P, Girod M, Ljungvall J, Ackermann D, Antalic S, Dorvaux O, Drouart A, Gall B, Gorgen A, Greenlees P T, Hauschild K, Herzberg R -D, Hessberger F P, Jakobsson U, Jones P, Julin R, Juutinen S, Ketelhut S, Korten W, Leino M, Lopez-Martens A, Nyman M, Obertelli A, Pakarinen J, Papadakis P, Parr E, Peura P, Piot J, Rahkila P, Rostron D, Ruotsalainen P, Sáren J, Scholey C, Sorri J, Uusitalo J, Venhart M and Zielinska M 2012 Investigation of high-K States in ${ }^{252}$ No Phys. Rev. C86 044318

[294] Jeppesen H B, Dragojevic I, Clark R M, Gregorich K E, Ali M N, Allmond J M, Beausang C W, Bleuel D L, Cromaz M, Deleplanque M A, Ellison P A, Fallon P, Garcia M A, Gates J M, Greene J P, Gros S, Lee I Y, Liu H L, Macchiavelli A O, Nelson S L, Nitsche H, Pavan J R, Stavsetra L, Stephens F S, Wiedeking M, Wyss R and Xu F R 2009 Multi-quasiparticle states in ${ }^{256}$ Rf Phys. Rev. C79 031303

[295] Robinson A P, Khoo T L, Seweryniak D, Ahmad I, Asai M, Back B B, Carpenter M P, Chowdhury P, Davids C N, Greene J, Greenlees P T, Hauschild K, Heinz A, Herzberg R -D, Janssens R V F, Jenkins D G, Jones G D, Ketelhut S, Kondev F G, Lauritsen T, Lister C J, Lopez-Martens A, Marley P, McCutchan E, Papadakis P, Peterson D, Qian J, Rostron D, Shirwadkar U, Stefanescu I, Tandel S K, Wang X and Zhu S 2011 Search for a 2-quasiparticle high-K isomer in ${ }^{256} \mathrm{Rf}$ Phys. Rev. C83 064311

[296] Rissanen J, Clark R M, Gregorich K E, Gates J M, Campbell C M, Crawford H L, Cromaz M, Esker N E, Fallon P, Forsberg U, Gothe O, Lee I-Y, Liu H L, Machiavelli A O, Mudder P, Nitsche H, Pang G, Rice A, Rudolph D, Stoyer M A, Wiens A and Xu F R 2013 Decay of the high-K isomeric state to a rotational band in ${ }^{257} \mathrm{Rf}$ Phys. Rev. C88 044313

[297] Lopez-Martens A, Hauschild K, Yeremin A V, Dorvaux O, Belozerov A V, Briançon Ch, Chelnokov M L, Chepigin V I, Curien D, Désesquelles P, Gall B, Gorshkov V A, Guttormsen M, Hanappe F, Kabachenko A P, Khalfallah F, Korichi A, Larsen A C, Malyshev O N, Minkova A, Oganessian Yu Ts, Popeko A G, Rousseau M, Rowley N, Sagaidak R N, Sharo S, Shutov A V, Siem S, Stuttgé L, Svirikhin A I, Syed N U H and Theisen Ch 2007 Isomeric states in ${ }^{253}$ No Eur. Phys. J. A32 245-50

[298] Lopez-Martens A, Wiborg-Hagen T, Hauschild K, Chelnokov M L, Chepigin V I, Curien D, Dorvaux O, Drafta G, Gall B, Görgen A, Guttormsen M, Isaev A V, Izosimov I N, Kabachenko 
A P, Katrasev D E, Kutsarova T, Kuznetsov A N, Larsen A C, Malyshev O N, Minkova A, Mullins S, Nyhus H T, Pantelica D, Piot J, Popeko A G, Saro S, Scintee N, Siem S, Syed N U H, Sokol E A, Svirikhin A I and Yeremin A V 2011 Spectroscopy of ${ }^{253}$ No and its daughters Nucl. Phys. A852 15-35

[299] Heßberger F P 2007 Nuclear Structure Investigations in the Region of Superheavy Nuclei Phys. At. Nucl. 70144551

[300] Qian J, Heinz A, Khoo T L, Janssens R V F, Peterson D, Seweryniak D, Ahmad I, Asai M, Back B B, Carpenter M P, Garnsworthy A B, Greene J P, Hecht A A, Jiang C L, Kondev F G, Lauritsen T, Lister C J, Robinson A, Savard G, Scott R, Vondrasek R, Wang X, Winkler R and Zhu S 2009 Spectroscopy of ${ }^{257}$ Rf Phys. Rev. C79 064319

[301] Berryman J S, Clark R M, Gregorich K E, Allmond J M, Bleuel D L, Cromaz M, Dragojevic I, Dvorak J, Ellison P A, Fallon P, Garcia M A, Gros S, Lee I Y, Macchiavelli A O, Paschalis S, Petri M, Qian J, Stoyer M A and Wiedeking M 2010 Electromagnetic decays of excited states in ${ }^{261} \mathrm{Sg}(\mathrm{Z}=106)$ and ${ }^{257} \mathrm{Rf}(\mathrm{Z}=104)$ Phys. Rev. C81 064325

[302] Antalic S, Heßberger F P, Hofmann S, Ackermann D, Heinz S, Kindler B, Kojouharov I, Kuusiniemi P, Leino M, Lommel B, Mann R, Nishio K, Sáro S, Streicher B, Sulignano B and Venhart M 2008 Decay studies of neutron-deficient lawrencium isotopes Eur. Phys. J. A38 219-26

[303] Hauschild K, Lopez-Martens A, Yeremin A V, Dorvaux O, Antalic S, Belozerov A V, Briançon Ch, Chelnokov M L, Chepigin V I, Curien D, Gall B, Görgen A, Gorshkov V A, Guttormsen M, Hanappe F, Kabachenko A P, Khalfallah F, Larsen A C, Malyshev O N, Minkova A, Popeko A G, Rousseau M, Rowley N, Sharo S, Shutov A V, Siem S, Stuttgé L, Svirikhin A I, Syed $\mathrm{N} \mathrm{U}$ H, Theisen Ch and Venhart M 2008 High-K, $\mathrm{t}_{1 / 2}=1.4(1) \mathrm{ms}$, isomeric state in ${ }^{255} \mathrm{Lr}$ Phys. Rev. C78 021302

[304] Jeppesen H B, Clark R M, Gregorich K E, Afanasjev A V, Ali M N, Allmond J M, Beausang C W, Cromaz M, Deleplanque M A, Dragojevic I, Dvorak J, Ellison P A, Fallon P, Garcia M A, Gates J M, Gros S, Lee I Y, Macchiavelli A O, Nelson S L, Nitsche H, Stavsetra L, Stephens F S and Wiedeking M 2009 High-K Multi-Quasiparticle States and Rotational Bands in ${ }_{103}^{255} \mathrm{Lr}$ Phys. Rev. C80 034324

[305] Kondev F G, Dracoulis G D, Khoo T -L, Lane G J, Byrne A P, Kibédi T, Ahmad I, Back B, Carpenter M P, Janssens R V F, Lauritsen T, Lister C J, Peterson D, Seweryniak D, Zhu S, Chowdhury P and Tandel S K 2008 Studies of multi-quasiparticle K-isomers in rare-earth and trans-fermium nuclei International Conference on Nuclear Data for Science and Technology, Nice, FRANCE, APR 22-27, 200, Proceedings (Editors: Bersillon, $O$ and Gunsing, $F$ and Bauge, E and Jacqmin, $R$ and Leray, S) Vol 1, 61-4

[306] Liu H L, Xu F R, Walker P M and Bertulani C A 2011 Effects of High-order Deformation in High-K Isomers in Superheavy Nuclei Phys. Rev. C83 011303(R)

[307] Chasman R R, Ahmad I, Friedman A M and Erskine J R 1977 Survey of single-particle states in the mass region $A>228$ Rev. Mod. Phys. 49 833-891

[308] Möller P, Nix J R, Myers W D and Swiatecki W J 1995 Nuclear ground-state masses and deformations At. Data and Nucl. Data Tables 59 185-381

[309] Liu H L, Walker P M and Xu F R 2014 Favored configurations for four-quasiparticle K isomerism in the heaviest nuclei Phys. Rev. C89 044304

[310] Prassa V, Lu B N, Niksić T, Ackermann D and Vretenar D 2015 High-K isomers in transactinide nuclei close to $\mathrm{N}=162$ Phys. Rev. C91 034324

[311] Hofmann S, Hessberger F P, Ackermann D, Antalic S, Cagarda P, Cwiok S, Kindler B, Kojouharova J, Lommel B, Mann R, Munzenberg G, Popeko A G, Saro S, Schott H J and Yeremin A V 2001 The new isotope ${ }^{270} 110$ and its decay products ${ }^{266} \mathrm{Hs}$ and ${ }^{262} \mathrm{Sg}$ Eur. Phys. J. A10 5-10

[312] Ackermann D, Hessberger F P, Antalic S, Block M, Burkhard H G, Comas V F, Greenlees P, Heinz S, Hofmann S, Ketelhut S, Khuyagbaatar J, Kindler B, Kojouharov I, Mazzocco M, 
Leino M, Lommel B, Mann R, Mauer J, Popeko A G, Sorri J, Uusitalo J and Yeremin A V 2011 Discovery of a K-Isomer in ${ }^{266} \mathrm{Hs}$ GSI Sci. Rep. 2011208

[313] Ackermann D $2012{ }^{270}$ Ds and its decay products - K-isomers, $\alpha$-sf competition and masses Proceedings of Science Bormio2012030

[314] David H M, Chen J, Seweryniak D, Kondev F G, Gates J M, Gregorich K E, Ahmad I, Albers M, Alcorta M, Back B B, Baartman B, Bertone P F, Bernstein L A, Campbell C M, Carpenter M P, Chiara C J, Clark R M, Cromaz M, Doherty D T, Dracoulis G D, Esker N E, Fallon P, Gothe O R, Greene J P, Greenlees P T, Hartley D J, Hauschild K, Hoffman C R, Hota S S, Janssens R V F, Khoo T L, Konki J, Kwarsick J T, Lauritsen T, Macchiavelli A O, Mudder P R, Nair C, Qiu Y, Rissanen J, Rogers A M, Ruotsalainen P, Savard G, Stolze S, Wiens A and Zhu S 2015 Decay and Fission Hindrance of Two- and Four-Quasiparticle K Isomers in ${ }^{254} \mathrm{Rf}$ Phys. Rev. Lett. 115 132502

[315] Xu F R, Zhao E G, Wyss R and Walker P M 2004 Enhanced Stability of Superheavy Nuclei Due to High-Spin Isomerism Phys. Rev. Lett. 92 252501

[316] Peterson D, Back B B, Janssens R V F, Khoo T L, Lister C J, Seweryniak D, Ahmad I, Carpenter M P, Davids C N, Hecht A A, Jiang C L, Lauritsen T, Wang X, Zhu S, Kondev F G, Heinz A, Qian J, Winkler R, Chowdhury P, Tandel S K and Tandel U S 2006 Decay modes of ${ }^{250}$ No Phys. Rev. C74 014316

[317] Baran A and Lojewski Z 1986 Spontaneous Fission of Isomeric States in ${ }^{250} \mathrm{Fm}$ and ${ }^{254}$ No Phys. Lett. B176 7-8

[318] Baran A and Lojewski Z 1987 Spontaneous Fission of Isomeric States of Actinide Nuclei Nucl. Phys. A475 327-37

[319] Lazarev Yu A 1987 Influence of Pairing Correlations on the Probability and Dynamics of Tunnelling Through the Barrier in Fission and Fusion of Complex Nuclei Phys. Scr. 35 255-66

[320] Walker P M, Xu F R, Liu H L and Sun Y 2012 On the possibility of enhanced fission stability for broken-pair excitations J. Phys. G 39105106

[321] Sandulescu A and Mirea M 2014 Cold fission from isomeric states of superheavy nuclei Eur. Phys. J. A50 110

[322] Sadhukhan J, Dobaczewski J, Nazarewicz W, Sheikh J A and Baran A 2014 Pairing-induced speedup of nuclear spontaneous fission Phys. Rev. C90 061304(R)

[323] Delion D S, Liotta R J and Wyss R 2007 Alpha-Decay of High-Spin Isomers in Superheavy Nuclei Phys. Rev. C76 044301

[324] Rissanen J, Clark R M, Macchiavelli A O, Fallon P, Campbell C M and Wiens A 2014 Effect of the reduced pairing interaction on $\alpha$-decay half-lives of multi-quasiparticle isomeric states Phys. Rev. C90 044324

[325] Walker P M, Lalkovski S and Stevenson P D 2010 Configuration dependence of K-forbidden transition rates from three-quasiparticle isomers Phys. Rev. C81 041304(R)

[326] Dracoulis G D, Lane G J, Kondev F G, Byrne A P, Kibédi T, Watanabe H, Ahmad I, Carpenter M P, Freeman S J, Janssens R V F, Hammond N J, Lauritsen T, Lister C J, Mukherjee G, Seweryniak D, Chowdhury P and Tandel S K 2005 Structure of two-, four-, and six-quasiparticle isomers in ${ }^{174} \mathrm{Yb}$ and K-forbidden decays Phys. Rev. C71 044326; Erratum Phys. Rev. C73 019901

[327] Hult M, Gasparro J,Marissens G, Lindahl P, Wätjen U, Johnston P N, Wagemans C and Köhler M 2006 Underground search for the decay of ${ }^{180} \mathrm{Ta}^{m}$ Phys. Rev. C74 054311

[328] Schlegel Ch, von Neumann-Cosel P, de Boer J, Gerl J, Kaspar M, Kozhoukharov I, Loewe M, Maier H J, Napiorkowski P J, Peter I, Rejmund M, Richter A, Schaffner H, Srebrny J, Würkner $\mathrm{M}$, Wollersheim H J, and the EBGSI96-Collaboration 2001 Depopulation of the $\mathrm{J}^{\pi}=9^{-}$Isomer in ${ }^{180} \mathrm{Ta}$ to the $\mathrm{J}^{\pi}=1^{+}$Ground State by Coulomb Excitation Eur. Phys. J. A10 135-8

[329] Hayes A B, Cline D, Moody K J, Ragnarsson I, Wu C Y, Becker J A, Carpenter M P, Carroll J J, Gohlke D, Greene J P, Hecht A A, Janssens R V F, Karamian S A, Lauritsen T, Lister C J, Macri R A, Propri R, Seweryniak D, Wang X, Wheeler R and Zhu S 2010 Coulomb 
excitation of a ${ }^{242} \mathrm{Am}$ isomeric target: E2 and E3 strengths, rotational alignment, and collective enhancement Phys. Rev. C82 044319

[330] Deylitz S, Valnion B D, El Abiary K, de Boer J, Gollwitzer N, Hertenberger R, Graw G, Kulessa R, Briançon Ch, Le Du D, Meunier R, Hussonnois M, Constantinescu O, Fortier S, Kim J B, Rosier L H, Rotbard G, Oganessian Yu Ts, Karamian S A, Wollersheim H J, Folger H, Gerl J, Happ Th and Hategan C 1996 Inelastic deuteron scattering from the high-spin isomer ${ }^{178} \mathrm{Hf}^{\mathrm{m} 2}$ $\left(16^{+}\right)$Phys. Rev. C53 1266-72

[331] Rotbard G, Berrier-Ronsin G, Constantinescu O, Fortier S, Gales S, Hussonnois M, Kim J B, Maison J M, Rosier L -H, Vernotte J, Briançon Ch, Kulessa R, Oganessian Yu Ts and Karamian S A 1993 Blocking effect on the $16^{+} \rightarrow 16^{+}$(p,t) transition on the isomeric ${ }^{178} \mathrm{Hf}^{m 2}$ target Phys. Rev. C48 R2148-50

[332] Muradian G V, Shatrov O Ya, Voskanian M A, Yastrebova L P, Volkov V L, Briançon Ch, Hussonnois M, Oganessian Yu Ts, Karamian S A and Constantinescu O 2003 Search for Neutron Resonances in the ${ }^{178 m 2} \mathrm{Hf}$ Isomer and Their Investigation Phys. At. Nucl. 66 6-16

[333] Karamian S A and Carroll J J 2011 Cross section for inelastic neutron "acceleration" by ${ }^{178} \mathrm{Hf}^{\mathrm{m} 2}$ Phys. Rev. C83 024604

[334] Roig O, Bélier G, Daugas J -M, Delbourgo P, Maunoury L, Méot V, Morichon E, Sauvestre J -E, Aupiais J, Boulin Y, Fioni G, Letourneau A, Marie F and Ridikas D 2004 High spin K isomeric target of ${ }^{177 m} \mathrm{Lu}$ Nucl. Instrum. Meth. A521 5-11

[335] Roig O, Meot V, Rosse B, Belier G, Daugas J -M, Letourneau A, Menelle A and Morel P 2011 Direct evidence for inelastic neutron "acceleration" by ${ }^{177} \mathrm{Lu}^{m}$ Phys. Rev. C83 064617

[336] Kondurov I A, Korothikh E M, Petrov Y V and Shuljak G I 1981 Acceleration of thermal neutrons by isomeric nuclei $\left({ }^{180} \mathrm{Hf}^{m}\right)$ Phys. Lett. B106 383-5

[337] Rykaczewski K, Grzywacz R, Lewitowicz M and Pfützner M 1998 New $\mu$ s-Isomers and Isomeric Beams Nucl. Phys. A630 30\%c-15c

[338] Walker P M 2006 Isomer beams Int. J. Mod. Phys. E15 1637-44

[339] Ishii T, Itoh M, Ishii M, Makishima A, Ogawa M, Hossain I, Hayakawa I and Kohno T 1997 Isomer-Scope: A new instrument for in-beam $\gamma$-ray spectroscopy through deep inelastic collisions Nucl. Instrum. Meth. Phys. Res. A395 210-16

[340] Morikawa T, Gono Y, Morita K, Kishida T, Murakami T, Ideguchi E, Kumagai H, Liu G H, Ferragut A, Yoshida A, Zhang Y H, Oshima M, Sugawara M, Kusakari H, Ogawa M, Nakajima M, Tsuchida H, Mitarai S, Odahara A, Kidera M, Shibata M, Kim J C, Chae S J, Hatsukawa $\mathrm{Y}$ and Ishihara M 1995 Coulomb Excitation of ${ }^{174} \mathrm{Hf}$ K-Isomer. $\gamma$-Ray Spectroscopy with HighSpin Isomer Beam Phys. Lett. B350 169-72

[341] Gono Y, Murakami T, Morikawa T, Ferragut A, Zhang Y H, Morita K, Yoshida A, Kumagai H, Oshima M, Kusakari H, Sugawara M, Ogawa M, Nakajima M, Mitarai S, Odahara A, Ideguchi E, Shizuma T, Kidera M, Kim J C, Chae S J and Min B J 1993 High-Spin Isomers and High-Spin Isomer Beams Nucl. Phys. A557 341c-6c

[342] Gono Y, Morikawa T, Kishida T, Morita K, Odahara A, Ideguchi E, Murakami T, Oshima M, Sugawara M, Kusakari H, Kumagai H, Tsuchida H, Ogawa M, Kidera M, Shibata M, Miyazaki K, Mitarai S, Zhang Y H, Ferragut A, Liu G H, Hatsukawa Y, Kim J C, Shin S A and Ishihara M 1995 Gamma-Ray Spectroscopy with High-Spin Isomer Beams Nucl. Phys. A588 241c-6c

[343] Watanabe H, Asahi K, Kishida T, Ueno H, Sato W, Yoshimi A, Kobayashi Y, Kameda D, Miyoshi H, Fukuchi T, Wakabayashi Y, Sasaki T, Kibe M, Hokoiwa N, Odahara A, Cederwall B, Lagergren K, Podolyák Zs, Ishihara M and Gono Y 2004 Application of the high-spin isomer beams to the secondary fusion reaction and the measurement of g-factor Nucl. Phys. A746 $540 c-3 c$

[344] Rowley N, Leigh J R, Wei J X and Lindsay R 1993 Obtaining average angular momenta from fusion excitation functions near the Coulomb barrier Phys. Lett. B314 179-84

[345] Butt R D, Dasgupta M, Gontchar I, Hinde D J, Mukherjee A, Berriman A C, Morton C R, Newton J O, Stuchbery A E and Lestone J P 2002 Effects on Finite Ground-State Spin on 
Fission Fragment Angular Distributions following Collisions with Spherical or Deformed Nuclei Phys. Rev. C65 044606; Erratum Phys. Rev. C65 069904

[346] Carroll J J, Karamian S A, Rivlin L A and A. A. Zadernovsky A A 2001 X-ray driven gamma emission, Hyperfine Interact. 135 3-50

[347] Ledingham K W D, McKenna P and Singhal R P 2003 Applications for Nuclear Phenomena Generated by Ultra-Intense Lasers Science 300 1107-11

[348] Liao Wen-Te, Adriana Pálffy A and Keitel C H 2013 Three-beam setup for coherently controlling nuclear-state population Phys. Rev. C87 054609

[349] Carroll J J 2007 Nuclear structure and the search for induced energy relase from isomers Nucl. Instrum. Meth. Phys. Res. B261 960-4

[350] Walker P M 2002 Mechanisms of K-mixing: The role of octupole vibrations in ${ }^{180} \mathrm{Ta}$ Hyperfine Interact. $143143-51$

[351] Collins C B, Davanloo F, Iosif M C, Dussart R, Hicks J M, Karamian S A, Ur C A, Popescu I I, Kirischuk V I, Carroll J J, Roberts H E, McDaniel P and Crist C E 1999 Accelerated Emission of Gamma Rays from the 31-yr Isomer of ${ }^{178} \mathrm{Hf}$ Induced by X-Ray Irradiation Phys. Rev. Lett. 82 $695-8$

[352] Collins C B, Zoita N C, Davanloo F, Yoda Y, Uruga T, Pouvesle J M and Popescu II 2005 Nuclear resonance spectroscopy of the 31-yr isomer of ${ }^{178} \mathrm{Hf}$ Laser Phys. Lett. 2 162-7

[353] Ahmad I, Banar J C, Becker J A, Bredeweg T A, Cooper J R, Gemmell D S, Kraemer A, Mashayekhi A, McNabb D P, Miller G G, Moore E F, Palmer P, Pangault L N, Rundberg R S, Schiffer J P, Shastri S D, Wang T -F and Wilhelmy J B 2005 Search for x-ray induced decay of the 31-yr isomer of ${ }^{178} \mathrm{Hf}$ using synchrotron radiation Phys. Rev. C71 024311

[354] Carroll J J, Karamian S A, Propri R, Gohlke D, Caldwell N, Ugorowski P, Drummond T, Lazich J, Roberts H, Helba M, Zhong Z, Tang M -T, Lee J -J and Liang K 2009 Search for low-energy induced depletion of ${ }^{178} \mathrm{Hf}^{m 2}$ at the SPring-8 synchrotron Phys. Lett. B679 203-8

[355] Sun Y, Zhou X -R, Long G -L, Zhao E -G and Walker P M 2004 Nuclear structure of ${ }^{178} \mathrm{Hf}$ related to the spin-16, 31-year isomer Phys. Lett. B589 83-8

[356] Hayes A B, Cline D, Wu C Y, Ai J, Amro H, Beausang C, Casten R F, Gerl J, Hecht A A, Heinz A, Hughes R, Janssens R V F, Lister C J, Macchiavelli A O, Meyer D A, Moore E F, Napiorkowski P, Pardo R C, Schlegel Ch, Seweryniak D, Simon M W, Srebrny J, Teng R, Vetter K and Wollersheim H J 2006 Breakdown of K Selection in ${ }^{178}$ Hf Phys. Rev. Lett. 96 042505

[357] Hayes A B, Cline D, Wu C Y, Ai J, Amro H, Beausang C, Casten R F, Gerl J, Hecht A A, Heinz A, Hua H, Hughes R, Janssens R V F, Lister C J, Macchiavelli A O, Meyer D A, Moore E F, Napiorkowski P, Pardo R C, Schlegel Ch, Seweryniak D, Simon M W, Srebrny J, Teng R, Vetter K and Wollersheim H J 2007 Spin dependence of K mixing, strong configuration mixing, and electromagnetic properties of ${ }^{178} \mathrm{Hf}$ Phys. Rev. C75 034308

[358] Hayes A B, Cline D, Wu C Y, Hurst A M, Carpenter M P, Greene J P, Janssens R V F, Lauritsen T, Seweryniak D, Zhu S, Karamian S A, Walker P M, Swan T P D, Rigby S V, Cullen D M, Lumley N M, Mason P, Carroll J J, Detwiler B, Harle T, Mills I and Trees G 2011 New structures in ${ }^{178} \mathrm{Hf}$ and Coulomb excitation of isomers Int. J. Mod. Phys. E20, 474-81

[359] Karpeshin F F, Trzhaskovskaya M B and Zhang J 2009 Prospect of triggering the ${ }^{178 m 2} \mathrm{Hf}$ isomer and the role of resonance conversion Eur. Phys. J. A39 341-8

[360] Kishimoto S, Yoda Y, Seto M, Kobayashi Y, Kitao S, Haruki R, Kawauchi T, Fukutani K and Okano T 2000 Observation of Nuclear Excitation by Electron Transition in ${ }^{197} \mathrm{Au}$ with Synchrotron X Rays and an Avalanche Photodiode Phys. Rev. Lett. 85 1831-4

[361] Gosselin G, Méot V and Morel P 2007 Modified nuclear level lifetime in hot dense plasmas Phys. Rev. C76 044611

[362] Gosselin G and Morel P 2004 Enhanced nuclear level decay in hot dense plasmas Phys. Rev. C70 064603

[363] Granja C, Kuba J, Haiduk A and Renner O 2007 Survey of nuclei for low-energy nuclear excitation 
in laser-produced plasma Nucl. Phys. A784 1-12

[364] Hasegawa M, Sun Y, Tazaki S, Kaneko K and Mizusaki T 2011 Characteristics of the 21/2+ isomer in ${ }^{93} \mathrm{Mo}$ : Toward the possibility of enhanced nuclear isomer decay Phys. Lett. B696 197-200

[365] Gunst J, Litvinov Y A, Keitel C H and Pálffy A 2014 Dominant Secondary Nuclear Photoexcitation with the X-Ray Free-Electron Laser Phys. Rev. Lett. 112082501

[366] Stefanescu I, Georgiev G, Ames F, Äystö J, Balabanski D L, Bollen G, Butler P A, Cederkäll J, Champault N, Davinson T, De Maesschalck A, Delahaye P, Eberth J, Fedorov D, Fedosseev V N, Fraile L M, Franchoo S, Gladnishki K, Habs D, Heyde K, Huyse M, Ivanov O, Iwanicki J, Jolie J, Jonson B, Kröll Th, Krücken R, Kester O, Köster U, Lagoyannis A, Liljeby L, Lo Bianco G, Marsh B A, Niedermaier O, Nilsson T, Oinonen M, Pascovici G, Reiter P, Saltarelli A, Scheit H, Schwalm D, Sieber T, Smirnova N, Van De Walle J, Van Duppen P, Zemlyanoi S, Warr N, Weisshaar D and Wenander F 2007 Coulomb Excitation of ${ }^{68,70} \mathrm{Cu}$ : First Use of Postaccelerated Isomeric Beams Phys. Rev. Lett. 98 122701

[367] Chen L, Plass W R, Geissel H, Knöbel R, Kozhuharov C, Litvinov Yu A, Patyk Z, Scheidenberger C, Siegień-Iwaniuk K, Sun B, Weick H, Beckert K, Beller P, Bosch F, Boutin D, Caceres L, Carroll J J, Cullen D M, Cullen I J, Franzke B, Gerl J, Górska M, Jones G A, Kishada A, Kurcewicz J, Litvinov S A, Liu Z, Mandal S, Montes F, Münzenberg G, Nolden F, Ohtsubo T, Podolyák Zs, Propri R, Rigby S, Saito N, Saito T, Shindo M, Steck M, Walker P M, Williams S, Winkler M, Wollersheim H -J and Yamaguchi T 2012 New results on mass measurements of stored neutron-rich nuclides in the element range from $\mathrm{Pt}$ to $\mathrm{U}$ with the FRS-ESR facility at 360-400 MeV/u Nucl. Phys. A882 71-89

[368] Walker P M, Litvinov Yu A and Geissel H 2013 The ILIMA project at FAIR Int. J. Mass Spectr. 349-350 247-254

[369] Grawe H, Langanke K and Martínez-Pinedo G 2007 Nuclear structure and astrophysics Rep. Prog. Phys. 70 1525-82

[370] Hayakawa T, Kajino T, Chiba S and Mathews G J 2010 New estimate for the time-dependent thermal nucleosynthesis of ${ }^{180 \mathrm{~m}}$ Ta Phys. Rev. C81 052801(R)

[371] Gosselin G, Morel P and Mohr P 2010 Modification of nuclear transitions in stellar plasma by electronic processes: $\mathrm{K}$ isomers in ${ }^{176} \mathrm{Lu}$ and ${ }^{180} \mathrm{Ta}$ under s-process conditions Phys. Rev. C81 055808

[372] Kajino T, Matthews G J and Hawakawa T 2014 Neutrinos in core-collapse supernovae and nucleosynthesis J. Phys. G: Nucl. Part. Phys. 41044007

[373] Auerbach N and Zelevinsky V 2014 Decay through a doorway state and the puzzle of ${ }^{180}$ Ta Phys. Rev. C90 034315

[374] Heil M, Winckler N, Dababneh S, Käppeler F, Wisshak K, Bisterzo S, Gallion R, Davis A M and Rauscher T $2008{ }^{176} \mathrm{Lu} /{ }^{176} \mathrm{Hf}$ : A Sensitive Test of s-Process Temperature and Neutron Density in AGB Stars Astrophys. J. 673 434-44

[375] Walter S, Stedile F, Carroll J J, Fransen C, Friessner G, Hollmann N, von Garrel H, Jolie J, Karg O, Käppeler F, Kneissl U, Kohstall C, von Neumann-Cosel P, Linnemann A, Mücher D, Pietralla N, Pitz H H, Rusev G, Scheck M, Scholl C, Schwengner R, Werner V and Wisshak K 2007 Photon scattering experiments on the quasistable, odd-odd mass nucleus ${ }^{176}$ Lu Phys. Rev. C75 034301

[376] Clayton D D 1964 Cosmoradiogenic Chronologies of Nucleosynthesis Astrophys. J. 139 637-63

[377] Hayakawa T, Shizuma T, Kajino T, Chiba S, Shinohara N, Nakagawa T and Arima T 2005 New s-Process Path and Its Implications for a ${ }^{187}$ Re- ${ }^{187}$ Os Nucleo-Cosmochronometer Astrophys. J. 628 533-40

[378] Wiescher M, Käppeler F and Langanke K 2012 Critical Reactions in Contemporary Nuclear Astrophysics Annu. Rev. Astron. Astrophys. 50 165-210

[379] Aprahamian A, Langanke K and Wiescher M 2005 Nuclear Structure Aspects in Nuclear Astrophysics Prog. Part. Nucl. Phys. 54 535-613 
[380] Watanabe H, Lorusso G, Nishimura S, Otsuka T, Ogawa K, Xu Z Y, Sumikama T, Soderstrom P -A, Doornenbal P, Li Z, Browne F, Gey G, Jung H S, Taprogge J, Vajta Zs, Wu J, Yagi A, Baba H, Benzoni G, Chae K Y, Crespi F C L, Fukuda N, Gernhauser R, Inabe N, Isobe T, Jungclaus A, Kameda D, Kim G D, Kim Y K, Kojouharov I, Kondev F G, Kubo T, Kurz N, Kwon Y K, Lane G J, Moon C -B, Montaner-Piza A, Moschner K, Naqvi F, Niikura M, Nishibata H, Nishimura D, Odahara A, Orlandi R, Patel Z, Podolyák Zs, Sakurai H, Schaffner H, Simpson G S, Steiger K, Suzuki H, Takeda H, Wendt A and Yoshinaga K 2014 MonopoleDriven Shell Evolution below the Doubly Magic Nucleus ${ }^{132}$ Sn Explored with the Long-Lived Isomer in ${ }^{126} \mathrm{Pd}$ Phys. Rev. Lett. 113042502

[381] Simpson G S, Gey G, Jungclaus A, Taprogge J, Nishimura S, Sieja K, Doornenbal P, Lorusso G, Soderstrom P -A, Sumikama T, Xu Z Y, Baba H, Browne F, Fukuda N, Inabe N, Isobe T, Jung H S, Kameda D, Kim G D, Kim Y -K, Kojouharov I, Kubo T, Kurz N, Kwon Y K, Li Z, Sakurai H, Schaffner H, Shimizu Y, Suzuki H, Takeda H, Vajta Z, Watanabe H, Wu J, Yagi A, Yoshinaga K, Bonig S, Daugas J -M, Drouet F, Gernhauser R, Ilieva S, Kroll T, Montaner-Piza A, Moschner K, Mucher D, Naidja H, Nishibata H, Nowacki F, Odahara A, Orlandi R, Steiger K and Wendt A 2014 Yrast $6^{+}$Seniority Isomers of ${ }^{136,138}$ Sn Phys. Rev. Lett. 113132502

[382] Patel Z, Soderstrom P -A, Podolyák Zs, Regan P H, Walker P M, Watanabe H, Ideguchi E, Simpson G S, Liu H L, Nishimura S, Wu Q, Xu F R, Browne F, Doornenbal P, Lorusso G, Rice S, Sinclair L, Sumikama T, Wu J, Xu Z Y, Aoi N, Baba H, Bello Garrote F L, Benzoni G, Daido R, Fang Y, Fukuda N, Gey G, Go S, Gottardo A, Inabe N, Isobe T, Kameda D, Kobayashi K, Kobayashi M, Komatsubara T, Kojouharov I, Kubo T, Kurz N, Kuti I, Li Z, Matsushita M, Michimasa S, Moon C -B, Nishibata H, Nishizuka I, Odahara A, Sahin E, Sakurai H, Schaffner H, Suzuki H, Takeda H, Tanaka M, Taprogge J, Vajta Zs, Yagi A and Yokoyama R 2014 Isomer Decay Spectroscopy of ${ }^{164} \mathrm{Sm}$ and ${ }^{166} \mathrm{Gd}$ : Midshell Collectivity Around N=100 Phys. Rev. Lett. 113 132502

[383] Cutler C S, Hennkens H M,Sisay N, Huclier-Markai S and Jurisson S S 2013 Radiometals for Combined Imaging and Therapy Chem. Rev. 113 858-83

[384] Pedersen J, Back B B, Bernthal F M, Bjørnholm S, Borggreen J, Christensen O, Folkmann F, Herskind B, Khoo T L, Neiman M, Pühlhofer F and Sletten G 1977 Island of High-Spin Isomers Near $\mathrm{N}=82$ Phys. Rev. Lett. 39 990-3

[385] Davids C N 2003 Recoil Separators Nucl. Instrum. Meth. B204 124-8

[386] Pfützner M, Karny M, Grigorenko L V and Riisager K 2012 Radioactive decays at limits of nuclear stability Rev. Mod. Phys. 84 567-619

[387] Leino M 2003 Gas-filled separators - An overview Nucl. Instrum. Meth. B204 129-37

[388] Schmidt K -H, Simon R S, Keller J -G, Hessberger F P, Münzenberg G, Quint B, Clerc H -G, Schwab W, Gollerthan U and Sahm C -C 1986 Gamma-Spectroscopic Investigations in the Radiative Fusion Reaction ${ }^{90} \mathrm{Zr}+{ }^{90} \mathrm{Zr}$ Phys. Lett. B168 39-42

[389] Paul E S, Woods P J, Davinson T, Page R D, Sellin P J, Beausang C W, Clark R M, Cunningham R A, Forbes S A, Fossan D B, Gizon A, Gizon J, Hauschild K, Hibbert I M, James A N, LaFosse D R, Lazarus I, Schnare H, Simpson J, Wadsworth R and Waring M P 1995 In-Beam $\gamma$-Ray Spectroscopy Above ${ }^{100} \mathrm{Sn}$ using the New Technique of Recoil Decay Tagging Phys. Rev. C51 $78-87$

[390] Seweryniak D, Woods P J, Ressler J J, Davids C N, Heinz A, Sonzogni A A, Uusitalo J, Walters W B, Caggiano J A, Carpenter M P, Cizewski J A, Davinson T, Ding K Y, Fotiades N, Garg U, Janssens R V F, Khoo T L, Kondev F G, Lauritsen T, Lister C J, Reiter P, Shergur J, Wiedenhover I 2001 Rotational Bands in the Proton Emitter ${ }^{141}$ Ho Phys. Rev. Lett. 86 1458-61

[391] Lagergren K, Joss D T, Wyss R, Cederwall B, Barton C J, Eeckhaudt S, Grahn T, Greenlees P T, Hadinia B, Jones P M, Julin R, Juutinen S, Karlgren D, Kettunen H, Leino M, Leppanen A -P, Nieminen P, Nyman M, Page R D, Pakarinen J, Paul E S, Rahkila P, Scholey C, Simpson $\mathrm{J}$, Uusitalo J and Wiseman D R 2006 High-spin states in the proton-unbound nucleus ${ }^{161}$ Re Phys. Rev. C74 024316 
[392] Joss D T, Thomson J, Scholey C, Erturk S, Simpson J, Page R D, Bianco L, Cederwall B, Darby I G, Eeckhaudt S, Gomez Hornillos M B, Grahn T, Greenlees P T, Jones P M, Julin R, Juutinen S, Ketelhut S, Lagergren K, Leino M, Leppanen A -P, Nyman M, O'Donnell D, Pakarinen J, Paul E S, Rahkila P, Sandzelius M, Sorri J, Saren J, Uusitalo $\mathrm{J}$ and Venhart M 2008 Spectroscopy of the neutron-deficient isobars ${ }^{163} \mathrm{Re}$ and ${ }^{163} \mathrm{~W}$ using tagging techniques Proc.Nuclear Physics and Astrophysics: From Stable Beams to Exotic Nuclei, Cappadocia, Turkey, June 25-30, 2008, I.Boztosun, A.B.Balantekin, Eds., p.154 (2008); AIP Conf. Proc. 1072 154-9

[393] Sandzelius M, Scholey C, Cederwall B, Ganioglu E, Andgren K, Appelbe D E, Barton C J, Back T, Eeckhaudt S, Grahn T, Greenlees P T, Hadinia B, Johnson A, Jones P M, Joss D T, Julin R, Juutinen S, Kettunen H, Lagergren K, Leino M, Leppanen A -P, Nieminen P, Page R D, Pakarinen J, Perkowski J, Rahkila P, Simpson J, Uusitalo J, Van de Vel K, Warner D D, Wiseman D R and Wyss R 2007 First identification of excited states in ${ }^{169}$ Ir Phys. Rev. C75 054321

[394] Kondev F G, Carpenter M P, Janssens R V F, Abu Saleem K, Ahmad I, Amro H, Cizewski J A, Danchev M, Davids C N, Hartley D J, Heinz A, Khoo T L, Lauritsen T, Lister C J, Ma W C, Poli G L, Ressler J, Reviol W, Riedinger L L, Seweryniak D, Smith M B and Wiedenhover I 2001 Identification of Excited Structures in Proton Unbound Nuclei ${ }^{173,175,177} \mathrm{Au}$ : Shape co-existence and intruder bands Phys. Lett. B512 268-76

[395] Joss D T, Simpson J, Appelbe D E, Barton C J, Warner D D, Lagergren K, Cederwall B, Hadinia B, Eeckhaudt S, Grahn T, Greenlees P T, Jones P M, Julin R, Juutinen S, Kettunen H, Leino M, Leppanen A -P, Nieminen P, Pakarinen J, Perkowski J, Rahkila P, Scholey C, Uusitalo J, Van de Vel K, Page R D, Paul E S, Wiseman D R and Riley M A 2006 Yrast states and band crossings in the neutron-deficient platinum isotopes ${ }^{169-173} \mathrm{Pt}$ Phys. Rev. C74 014302

[396] Kondev F G, Carpenter M P, Janssens R V F, Abu Saleem K, Ahmad I, Alcorta M, Amro H, Caggiano J, Cizewski J A, Danchev M, Davids C N, Hartley D J, Heinz A, Herskind B, Kaye R A, Khoo T L, Lauritsen T, Lister C J, Ma W C, Poli G L, Ressler J, Reviol W, Riedinger L L, Seweryniak D, Siem S, Smith M B, Sonzogni A A, Varmette P G and Wiedenhover I 2001 First Observation of Excited Structures in Neutron Deficient, Odd-Mass Pt, Au and Hg Nuclei Nucl. Phys. A682 487c-92c

[397] Peura P, Scholey C, Joss D T, Juutinen S, Julin R, Back T, Cederwall B, Greenlees P T, Jakobsson U, Jones P, Judson D S, Ketelhut S, Labiche M, Leino M, Nyman M, O'Donnell D, Page R D, Rahkila P, Ruotsalainen P, Sandzelius M, Sapple P J, Saren J, Simpson J, Thomson J, Uusitalo J and Watkins H V 2014 Quasiparticle alignments and $\alpha$-decay fine structure of ${ }^{175} \mathrm{Pt}$ Phys. Rev. C89 024316

[398] O’Donnell D, Simpson J, Scholey C, Back T, Greenlees P T, Jakobsson U, Jones P, Joss D T, Judson D S, Julin R, Juutinen S, Ketelhut S, Labiche M, Leino M, Nyman M, Page R D, Peura P, Rahkila P, Ruotsalainen P, Sandzelius M, Sapple P J, Saren J, Thomson J, Uusitalo J and Watkins H V 2009 First observation of excited states in ${ }^{175} \mathrm{Hg}_{95}$ Phys. Rev. C79 051304

[399] Melerangi A, Appelbe D, Page R D, Boardman H J, Greenlees P T, Jones P, Joss D T, Julin R, Juutinen S, Kettunen H, Kuusiniemi P, Leino M, Muikku M H, Nieminen P, Pakarinen J, Rahkila P and Simpson J 2003 Shape isomerism and spectroscopy of ${ }^{177} \mathrm{Hg}$ Phys. Rev. C6 8 041301

[400] Kondev F G, Carpenter M P, Janssens R V F, Lister C J, Abu Saleem K, Ahmad I, Amro H, Caggiano J, Davids C N, Heinz A, Herskind B, Khoo T L, Lauritsen T, Ma W C, Ressler J J, Reviol W, Riedinger L L, Sarantites D G, Seweryniak D, Siem S, Sonzogni A A, Varmette P G and Wiedenhover I 2002 First Observation of Excited Structures in Neutron-Deficient ${ }^{179} \mathrm{Hg}$ : Evidence for multiple shape coexistence Phys. Lett. B528 221-7

[401] Taylor M J, Cullen D M, Procter M G, Smith A J, McFarlane A, Twist V, Alharshan G A, Ferreira L S, Maglione E, Auranen K, Grahn T, Greenlees P T, Hauschild K, Herzan A, Jakobsson U, Julin R, Juutinen S, Ketelhut S, Konki J, Leino M, Lopez-Martens A, Pakarinen J, Partanen 
J, Peura P, Rahkila P, Rinta-Antila S, Ruotsalainen P, Sandzelius M, Saren J, Scholey C, Sorri J, Stolze S, Uusitalo J and Doncel M 2015 Oblately deformed isomeric proton-emitting state in ${ }^{151} \mathrm{Lu}$ Phys. Rev. C91 044322

[402] Ding K Y, Cizewski J A, Seweryniak D, Amro H, Carpenter M P, Davids C N, Fotiades N, Janssens R V F, Lauritsen T, Lister C J, Nisius D, Reiter P, Uusitalo J, Wiedenhover I and Macchiavelli A O 2001 Excited States in ${ }^{155} \mathrm{Yb}$ and ${ }^{155,156,157} \mathrm{Lu}$ from Recoil-Decay Tagging Phys. Rev. C64 034315

[403] Seweryniak D, Uusitalo J, Bhattacharyya P, Carpenter M P, Cizewski J A, Ding K Y, Davids C N, Fotiades N, Janssens R V F, Lauritsen T, Lister C J, Macchiavelli A O, Nisius D, Reiter P, Walters W B and Woods P J 2005 Multiparticle configurations in N $=84$ isotones located at the proton drip line Phys. Rev. C71 054319

[404] Carroll R J, Page R D, Joss D T, Uusitalo J, Darby I G, Andgren K, Cederwall B, Eeckhaudt S, Grahn T, Gray-Jones C, Greenlees P T, Hadinia B, Jones P M, Julin R, Juutinen S, Leino M, Leppanen A -P, Nyman M, O'Donnell D, Pakarinen J, Rahkila P, Sandzelius M, Saren J, Scholey C, Seweryniak D and Simpson J 2014 Blurring the Boundaries: Decays of Multiparticle Isomers at the Proton Drip Line Phys. Rev. Lett. 112092501

[405] Specht H J, Weber J, Konecny E and Heunemann D 1972 Identification of a rotational band in the ${ }^{240} \mathrm{Pu}$ fission isomer Phys. Lett. B41 43-6

[406] Jones G 2002 Detection of Long-Lived Isomers in Super-Heavy Elements Nucl. Instrum. Meth. Phys. Res. A488 471-2

[407] Cullen D M, Amzal N, Boston A J, Butler P A, Keenan A, Paul E S, Scraggs H C, Bruce A M, Parry C M, Cocks J F C, Helariutta K, Jones P M, Julin R, Juutinen S, Kankaanpaa H, Kettunen H, Kuusiniemi P, Leino M, Muikku M and Savelius A 1998 Identification of the $\mathrm{K}^{\pi}=8^{-}$Rotational Band in ${ }^{138} \mathrm{Gd}$ Phys. Rev. C58 846-50

[408] Steer A N, Jenkins D G, Glover R, Nara Singh B S, Pattabiraman N S, Wadsworth R, Eeckhaudt S, Grahn T, Greenlees P T, Jones P, Julin R, Juutinen S, Leino M, Nyman M, Pakarinen J, Rahkila P, Saren J, Scholey C, Sorri J, Uusitalo J, Butler P A, Darby I G, Herzberg R -D, Joss D T, Page R D, Thomson J, Lemmon R, Simpson J and Blank B 2006 Recoil-beta tagging: A novel technique for studying proton-drip-line nuclei Nucl. Instrum. Meth. Phys. Res. A565 630-6

[409] Nara Singh B S, Steer A N, Jenkins D G, Wadsworth R, Bentley M A, Davies P J, Glover R, Pattabiraman N S, Lister C J, Grahn T, Greenlees P T, Jones P, Julin R, Juutinen S, Leino M, Nyman M, Pakarinen J, Rahkila P, Saren J, Scholey C, Sorri J, Uusitalo J, Butler P A, Dimmock M, Joss D T, Thomson J, Cederwall B, Hadinia B and Sandzelius M 2007 Coulomb shifts and shape changes in the mass 70 region Phys. Rev. C75 061301(R)

[410] Pfützner M, Regan P H, Walker P M, Caamano M, Gerl J, Hellstrom M, Mayet P, Schmidt K -H, Podolyák Zs, Mineva M N, Aprahamian A, Benlliure J, Bruce A M, Butler P A, Cortina Gil D, Cullen D M, Döring J, Enqvist T, Fox C, Garces Narro J, Geissel H, Gelletly W, Giovinazzo J, Gorska M, Grawe H, Grzywacz R, Kleinbohl A, Korten W, Lewitowicz M, Lucas R, Mach H, O'Leary C D, De Oliveira F, Pearson C J, Rejmund F, Rejmund M, Sawicka M, Schaffner H, Schlegel Ch, Schmidt K, Theisen Ch, Vives F, Warner D D, Wheldon C, Wollersheim H $\mathrm{J}$ and Wooding S 2002 Angular momentum population in the fragmentation of ${ }^{208} \mathrm{~Pb}$ at 1 $\mathrm{GeV} /$ nucleon Comprehensinsive isomer/spin analysis Phys. Rev. C65 064604

[411] Benlliure J, Schmidt K -H, Cortina-Gil D, Enqvist T, Farget F, Heinz A, Junghans A R, Pereira $\mathrm{J}$ and Taieb J 1999 Production of neutron-rich isotopes by cold fragmentation in the reaction ${ }^{197} \mathrm{Au}+$ Be at $950 \mathrm{~A} \mathrm{MeV} \mathrm{Nucl.} \mathrm{Phys.} \mathrm{A660} \mathrm{87-100}$

[412] Bacelar A M D, Bruce A M, Podolyák Zs, Al-Dahan N, Gorska M, Lalkovski S, Pietri S, Ricciardi M V, Algora A, Alkhomashi N, Benlliure J, Boutachkov P, Bracco A, Calore E, Casarejos E, Cullen I J, Deo A Y, Detistov P, Dombradi Zs, Domingo-Pardo C, Doncel M, Farinon F, Farrelly G F, Geissel H, Gelletly W, Gerl J, Goel N, Grebosz J, Hoischen R, Kojouharov I, Kurz N, Leoni S, Molina F, Montanari D, Morales A I, Musumarra A, Napoli D R, Nicolini R, 
Nociforo C, Prochazka A, Prokopowicz W, Regan P H, Rubio B, Rudolph D, Schmidt K -H, Schaffner H, Steer S J, Steiger K, Strmen P, Swan T P D, Szarka I, Valiente-Dobon J J, Verma S, Walker P M, Weick H and Wollersheim H J 2013 The population of metastable states as a probe of relativistic-energy fragmentation reactions Phys. Lett. B723 302-6

[413] Podolyák Zs, Steer S J, Pietri S, Xu F R, Liu H L, Regan P H, Rudolph D, Garnsworthy A B, Hoischen R, Gorska M, Gerl J, Wollersheim H J, Kurtukian-Nieto T, Benzoni G, Shizuma T, Becker F, Bednarczyk P, Caceres L, Doornenbal P, Geissel H, Grebosz J, Kelic A, Kojouharov I, Kurz N, Montes F, Prokopowicz W, Saito T, Schaffner H, Tashenov S, Heinz A, Pfützner M, Jungclaus A, Balabanski D L, Brandau C, Bruce A M, Catford W N, Cullen I J, Dombradi Zs, Estevez E, Gelletly W, Ilie G, Jolie J, Jones G A, Kmiecik M, Kondev F G, Krücken R, Lalkovski S, Liu Z, Maj A, Myalski S, Schwertel S, Walker P M, Werner-Malento E and Wieland O 2009 Weakly deformed oblate structures in ${ }_{76}^{198} \mathrm{Os}_{122}$ Phys. Rev. C79 031305(R)

[414] Podolyák Zs, Farrelly G F, Regan P H, Garnsworthy A B, Steer S J, Gorska M, Benlliure J, Casarejos E, Pietri S, Gerl J, Wollersheim H J, Kumar R, Molina F, Algora A, Alkhomashi N, Benzoni G, Blazhev A, Boutachkov P, Bruce A M, Caceres L, Cullen I J, Bacelar A M D, Doornenbal P, Estevez M E, Fujita Y, Gelletly W, Geissel H, Grawe H, Grebosz J, Hoischen R, Kojouharov I, Lalkovski S, Liu Z, Maier K H, Mihai C, Mucher D, Rubio B, Schaffner H, Tamii A, Tashenov S, Valiente-Dobon J J, Walker P M and Woods P J 2009 Phys. Lett. B672 116-9

[415] Caamano M, Walker P M, Regan P H, Pfützner M, Podolyák Zs, Gerl J, Hellstrom M, Mayet P, Mineva M N, Aprahamian A, Benlliure J, Bruce A M, Butler P A, Cortina Gil D, Cullen D M, Döring J, Enqvist T, Fox C, Garces Narro J, Geissel H, Gelletly W, Giovinazzo J, Gorska M, Grawe H, Grzywacz R, Kleinbohl A, Korten W, Lewitowicz M, Lucas R, Mach H, O'Leary C D, De Oliveira F, Pearson C J, Rejmund F, Rejmund M, Sawicka M, Schaffner H, Schlegel C, Schmidt K, Schmidt K -H, Stevenson P D, Theisen Ch, Vives F, Warner D D, Wheldon C, Wollersheim H J, Wooding S, Xu F and Yordanov O 2005 Isomers in neutron-rich A $\approx 190$ nuclides from ${ }^{208} \mathrm{~Pb}$ fragmentation Euro. Phys. J. A23 201-15

[416] Steer S J, Podolyák Zs, Pietri S, Gorska M, Grawe H, Maier K H, Regan P H, Rudolph D, Garnsworthy A B, Hoischen R, Gerl J, Wollersheim H J, Becker F, Bednarczyk P, Caceres L, Doornenbal P, Geissel H, Grebosz J, Kelic A, Kojouharov I, Kurz N, Montes F, Prokopwicz W, Saito T, Schaffner H, Tashenov S, Heinz A, Pfützner M, Kurtukian-Nieto T, Benzoni G, Jungclaus A, Balabanski D L, Bowry M, Brandau C, Brown A, Bruce A M, Catford W N, Cullen I J, Dombradi Zs, Estevez M E, Gelletly W, Ilie G, Jolie J, Jones G A, Kmiecik M, Kondev F G, Krücken R, Lalkovski S, Liu Z, Maj A, Myalski S, Schwertel S, Shizuma T, Walker P M, Werner-Malento E and Wieland O 2011 Isomeric states observed in heavy neutron-rich nuclei populated in the fragmentation of a ${ }^{208} \mathrm{~Pb}$ beam Phys. Rev. C84 044313

[417] Al-Dahan N, Podolyák Zs, Regan P H, Gorska M, Grawe H, Maier K H, Gerl J, Pietri S B, Wollersheim H J, Alkhomashi N, Deo A Y, Bacelar A M D, Farrelly G, Steer S J, Bruce A M, Boutachkov P, Domingo-Pardo C, Algora A, Benlliure J, Bracco A, Calore E, Casarejos E, Cullen I J, Detistov P, Dombradi Zs, Doncel M, Farinon F, Gelletly W, Geissel H, Goel N, Grebosz J, Hoischen R, Kojouharov I, Kurz N, Lalkovski S, Leoni S, Molina F, Montanari D, Morales A I, Musumarra A, Napoli D R, Nicolini R, Nociforo C, Prochazka A, Prokopowicz W, Rubio B, Rudolph D, Schaffner H, Strmen P, Szarka I, Swan T, Thomas J S, Valiente-Dobon J J, Verma S, Walker P M and Weick H 2009 Nuclear structure "southeast" of ${ }^{208} \mathrm{~Pb}$ : Isomeric states in ${ }^{208} \mathrm{Hg}$ and ${ }^{209} \mathrm{Tl}$ Phys. Rev. C80 061302(R)

[418] Al-Dahan N, Regan P H, Podolyák Zs, Walker P M, Alkhomashi N, Dracoulis G D, Farrelly G, Benlliure J, Pietri S B, Casten R F, Stevenson P D, Gelletly W, Steer S J, Garnsworthy A B, Casarejos E, Gerl J, Wollersheim H J, Grebosz J, Gorska M, Kojouharov I, Schaffner H, Algora A, Benzoni G, Blazhev A, Boutachkov P, Bruce A M, Cullen I J, Bacelar A M D, Deo A Y, Estevez M E, Fujita Y, Hoischen R, Kumar R, Lalkovski S, Liu Z, Mason P J, Mihai C, Molina F, Mucher D, Rubio B, Tamii A, Tashenov S, Valiente-Dobon J J and Woods P J 
2012 Multiple $\beta^{-}$decaying states in ${ }^{194}$ Re: Shape evolution in neutron-rich osmium isotopes Phys. Rev. C85 034301

[419] Pietri S, Regan P H, Podolyák Zs, Rudolph D, Steer S, Garnsworthy A B, Werner-Malento E, Hoischen R, Gorska M, Gerl J, Wollersheim H J, Kojouharov I, Schaffner H, Becker F, Bednarczyk P, Caceres L, Doornenbal P, Geissel H, Grebosz J, Kelic A, Kurz N, Montes F, Prokopowicz W, Saito T, Tashenov S, Heinz A, Pfützner M, Kurtukian-Nieto T, Benzoni G, Hellstrom M, Jungclaus A, Simpson J, Andersson L -L, Atanasova L, Balabanski D, Bentley M A, Blank B, Blazhev A, Brandau C, Brown J R, Bruce A M, Camera F, Catford W N, Cullen I J, Dombradi Zs, Estevez E, Fahlander C, Gelletly W, Ilie G, Johansson E K, Jolie J, Jones G A, Kmiecik M, Kondev F G, Lalkovski S, Liu Z, Maj A, Myalski S, Shizuma T, Simons A J, Schwertel S, Walker P M and Wieland O 2007 Recent results in fragmentation isomer spectroscopy with RISING Nucl. Instr. Meth. Phys. Res. B261 1079-83

[420] Bowry M, Podolyák Zs, Pietri S, Kurcewicz J, Bunce M, Regan P H, Farinon F, Geissel H, Nociforo C, Prochazka A, Weick H, Al-Dahan N, Alkhomashi N, Allegro P R P, Benlliure J, Benzoni G, Boutachkov P, Bruce A M, Bacelar A M D, Farrelly G F, Gerl J, Gorska M, Gottardo A, Grebosz J, Gregor N, Janik R, Knöbel R, Kojouharov I, Kubo T, Kurz N, Litvinov Yu A, Merchan E, Mukha I, Naqvi F, Pfeiffer B, Pfützner M, Plass W, Pomorski M, Riese B, Ricciardi M V, Schmidt K -H, Schaffner H, Scheidenberger C, Simpson E C, Sitar B, Spiller P, Stadlmann J, Strmen P, Sun B, Tanihata I, Terashima S, Valiente Dobon J J, Winfield J S, Wollersheim H -J and Woods P J 2013 Population of high-spin isomeric states following fragmentation of ${ }^{238} \mathrm{U}$ Phys. Rev. C88 024611

[421] Kameda D, Kubo T, Ohnishi T, Kusaka K, Yoshida A, Yoshida K, Ohtake M, Fukuda N, Takeda H, Tanaka K, Inabe N, Yanagisawa Y, Gono Y, Watanabe H, Otsu H, Baba H, Ichihara T, Yamaguchi Y, Takechi M, Nishimura S, Ueno H, Yoshimi A, Sakurai H, Motobayashi T, Nakao T, Mizoi Y, Matsushita M, Ieki K, Kobayashi N, Tanaka K, Kawada Y, Tanaka N, Deguchi S, Satou Y, Kondo Y, Nakamura T, Yoshinaga K, Ishii C, Yoshii H, Miyashita Y, Uematsu N, Shiraki Y, Sumikama T, Chiba J, Ideguchi E, Saito A, Yamaguchi T, Hachiuma I, Suzuki T, Moriguchi T, Ozawa A, Ohtsubo T, Famiano M A, Geissel H, Nettleton A S, Tarasov O B, Bazin D, Sherrill B M, Manikonda S L and Nolen J A 2012 Observation of new microsecond isomers among fission products from in-flight fission of $345 \mathrm{MeV} /$ nucleon ${ }^{238} \mathrm{U}$ Phys. Rev. C86 054319

[422] Dracoulis G D, Byrne A P, Kibédi T, McGoram T R, and Mullins S M 1997 Incomplete fusion as a spectroscopic tool J. Phys. G23 1191-202

[423] Dracoulis G D, Mullins S M, Byrne A P, Kondev F G, Kibédi T, Bayer S, Lane G J, McGoram T R and Davidson P M 1998 Intrinsic states and collective structures in ${ }^{180} \mathrm{Ta}$ Phys. Rev. C58 $1444-66$

[424] McGoram T R, Dracoulis G D, Kibédi T, Byrne A P, Bark R A, Baxter A M and Mullins S M 2000 Normal and anomalous K-hindered decays from four-quasiparticle isomers in ${ }^{176} \mathrm{Lu}$ Phys. Rev. C62 031303(R)

[425] Podolyák Zs, Walker P M, Mach H, de France G, Sletten G, Azaiez F, Casandjian J M, Cederwall B, Cullen D M, Dombradi Zs, Dracoulis G D, Fraile L M, Franchoo S, Fynbo H, Gorska M, Kopatch Y, Lane G J, Mandal S, Milechina L, Molnar J, O'Leary C, Plociennik W, Pucknell V, Raddon P, Redon N, Ruchowska E, Stanoiu M, Tengblad O, Wheldon C and Wood R 2003 $\gamma$-ray spectroscopy with a ${ }^{8} \mathrm{He}$ beam Nucl. Instr. Meth. Phys. Res. A511 354-9

[426] Odahara A,Takashima A, Suga M, Tajiri K, Kurata K, Takatsu J, Ito K, Kenmoku Y, Yamaguchi K, Kazato M, Kura K, Shimoda T, Suzuki T, Watanabe H, Nishimura S, Gono Y, Ideguchi E, Go S, Wakabayashi Y, Morikawa T, Petrache C and Beaumel D 2010 Isomer Spectroscopy at RI Beam Line in RCNP Mod. Phys. Lett. A25 1951-54

[427] Wilczyǹski J 1973 Nuclear Molecules and Nuclear Friction Phys. Lett. B47 484-6

[428] Essel H, Hartel K, Henning W, Kienle P, Körner H J, Rehm K E, Sperr P and Wagner W 1979 Charge and Mass Transfer in the Reaction ${ }^{136} \mathrm{Xe}+{ }^{208} \mathrm{~Pb}$ at Energies Close to the Coulomb 
Barrier Z. Phys. A289 265-71

[429] Broda R, Quader M A, Daly P J, Janssens R V F, Khoo T L, Ma W C and Drigert M W 1990 Inelastic and Transfer Reactions in ${ }^{92} \mathrm{Mo}+255 \mathrm{MeV}^{60} \mathrm{Ni}$ Collisions Studied by $\gamma \gamma$ Coincidences Phys. Lett. B251 245-9

[430] Broda R, Zhang C T, Kleinheinz P, Menegazzo R, Maier K -H, Grawe H, Schramm M, Schubart R, Lach M and Hofmann S 1995 Collisions between ${ }^{106} \mathrm{Cd}$ and ${ }^{54} \mathrm{Fe}$ at $30 \mathrm{MeV}$ above the Coulomb barrier by high resolution $\gamma \gamma$ coincidences Phys. Rev. C49 R575-9

[431] Cocks J F C, Butler P A, Cann K J, Greenlees P T, Jones G D, Smith J F, Jones P M, Julin R, Juutinen S, Muller D, Piiparinen M, Savelius A, Broda R, Fornal B, Ahmad I, Blumenthal D J, Carpenter M P, Crowell B, Janssens R V F, Khoo T L, Lauritsen T, Nisius D, Asztalos S, Clark R M, Deleplanque M A, Diamond R M, Fallon P, Lee I Y, Macchiavelli A O, MacLeod R W, Stephens F S, Bhattacharyya P and Zhang C T 2000 Multi-Nucleon Transfer Reactions as a Tool for Spectroscopy of Heavy Nuclei J. Phys. G26 L23-32

[432] Broda R 2006 Spectroscopic studies with the use of deep-inelastic heavy-ion reactions J. Phys. G32 R151-92

[433] de Angelis G 2007 Nuclear structure at the limits. Exploring the changing of shell structure with modern $\gamma$-ray detectors Prog. Part. Nucl. Phys. 59 409-17

[434] Lunardi S, Lenzi S M, Della Vedova F, Farnea E, Gadea A, Marginean N, Bazzacco D, Beghini S, Bizzeti P G, Bizzeti-Sona A M, Bucurescu D, Corradi L, Deacon A N, de Angelis G, Fioretto E, Freeman S J, Ionescu-Bujor M, Iordachescu A, Mason P, Mengoni D, Montagnoli G, Napoli D R, Nowacki F, Orlandi R, Pollarolo G, Recchia F, Scarlassara F, Smith J F, Stefanini A M, Szilner S, Ur C A, Valiente-Dobon J J and Varley B J 2007 Spectroscopy of neutron-rich Fe isotopes populated in the ${ }^{64} \mathrm{Ni}+{ }^{238} \mathrm{U}$ reaction Phys. Rev. C76 034303

[435] Valiente-Dobon J J, Regan P H, Wheldon C, Wu C Y, Yoshinaga N, Higashiyama K, Smith J F, Cline D, Chakrawarthy R S, Chapman R, Cromaz M, Fallon P, Freeman S J, Gorgen A, Gelletly W, Hayes A, Hua H, Langdown S D, Lee I Y, Liang X, Macchiavelli A O, Pearson C J, Podolyák Zs, Sletten G, Teng R, Ward D, Warner D D and Yamamoto A D $2004{ }^{136} \mathrm{Ba}$ studied via deep-inelastic collisions: Identification of the $\left(\nu \mathrm{h}_{11 / 2}\right)_{10+}^{-2}$ isomer Phys. Rev. C69 024316

[436] Wheldon C, D'Alarcao R, Chowdhury P, Walker P M, Seabury E, Ahmad I, Carpenter M P, Cullen D M, Hackman G, Janssens R V F, Khoo T L, Nisius D, Pearson C J and Reiter P 1998 Opening up the $\mathrm{A} \approx 180 \mathrm{~K}$-Isomer Landscape: Inelastic excitation of new multi-quasiparticle yrast traps Phys. Lett. B425 239-45

[437] Wheldon C, Walker P M, D'Alarcao R, Chowdhury P, Pearson C J, Seabury E H, Ahmad I, Carpenter M P, Cullen D M, Hackman G, Janssens R V F, Khoo T L, Nisius D and Reiter P 1999 Identification of a High-K Isomer in Neutron-Rich ${ }^{185}$ Ta Eur. Phys. A5 353-5

[438] Wheldon C, Walker P M, Chowdhury P, Shestakova I, D'Alarcao R, Ahmad I, Carpenter M P, Cullen D M, Janssens R V F, Khoo T L, Kondev F G, Lister C J, Pearson C J, Podolyák Zs, Seweryniak D and Wiedenhoever I 2000 Inelastic Excitation of New High-Spin Yrast Isomers in ${ }^{180}$ Ta Phys. Rev. C62 057301

[439] Wheldon C, Walker P M, Chowdhury P, Shestakova I, D'Alarcao R, Ahmad I, Carpenter M P, Cullen D M, Janssens R V F, Khoo T L, Kondev F G, Lister C J, Pearson C J, Podolyák Zs, Seweryniak D and Wiedenhoever I 2001 First Observation of a Rotational Band in NeutronRich ${ }^{180} \mathrm{Lu}$ J. Phys. G27 L13-7

[440] Wheldon C, Valiente-Dobon J J, Regan P H, Pearson C J, Wu C Y, Smith J F, Macchiavelli A O, Cline D, Chakrawarthy R S, Chapman R, Cromaz M, Fallon P, Freeman S J, Gorgen A, Gelletly W, Hayes A, Hua H, Langdown S D, Lee I Y, Liang X, Podolyák Zs, Sletten G, Teng R, Ward D, Warner D D and Yamamoto A D 2004 Multi-quasiparticle states in ${ }^{184} \mathrm{~W}$ via multi-nucleon transfer Eur. Phys. J. A20 365-9

[441] D'Alarcao R, Chowdhury P, Seabury E H, Walker P M, Wheldon C, Ahmad I, Carpenter M P, Hackman G, Janssens R V F, Khoo T L, Lister C J, Nisius D, Reiter P, Seweryniak D and 
Wiedenhoever I 1999 High-K Isomers in Neutron-Rich Hafnium Nuclei At and Beyond the Stability Line Phys. Rev. C59 R1227-31

[442] Shestakova I, Mukherjee G, Chowdhury P, D'Alarcao R, Pearson C J, Podolyák Zs, Walker P M, Wheldon C, Cullen D M, Ahmad I, Carpenter M P, Janssens R V F, Khoo T L, Kondev F G, Lister C J, Seweryniak D and Wiedenhoever I 2001 Yrast Three-Quasiparticle K Isomers in Neutron-Rich ${ }^{181} \mathrm{Hf}$ Phys. Rev. C64 054307

[443] Dracoulis G D, Lane G J, Kondev F G, Watanabe H, Seweryniak D, Zhu S, Carpenter M P, Chiara C J, Janssens R V F, Lauritsen T, Lister C J, McCutchan E and Stefanescu I 2010 Two-quasiparticle structures and isomers in ${ }^{168} \mathrm{Er},{ }^{170} \mathrm{Er}$, and ${ }^{172} \mathrm{Er}$ Phys. Rev. C81 054313

[444] Hughes R O, Lane G J, Dracoulis G D, Byrne A P, Nieminen P, Watanabe H, Kondev F G, Carpenter M P, Janssens R V F, Lauritsen T, Seweryniak D, Zhu S and Chowdhury P 2012 High-spin structure, K isomers, and state mixing in the neutron-rich isotopes ${ }^{173} \mathrm{Tm}$ and ${ }^{175} \mathrm{Tm}$ Phys. Rev. C86 054314

[445] Dracoulis G D, Lane G J, Kondev F G, Byrne A P, Kibédi T, Watanabe H, Ahmad I, Carpenter M P, Freeman S J, Janssens R V F, Hammond N, Lauritsen T, Lister C J, Mukherjee G, Seweryniak D, Chowdhury P and Tandel S K 2005 Structure of two-, four-, and six-quasiparticle isomers in ${ }^{174} \mathrm{Yb}$ and K-forbidden decays Phys. Rev. C71 044326

[446] Kondev F G, Dracoulis G D, Lane G J, Ahmad I, Byrne A P, Carpenter M P, Chowdhury P, Janssens R V F, Kibédi T, Lauritsen T, Lister C J, Seweryniak D, Tandel S K and Zhu S 2009 Multi-quasiparticle isomers in ${ }^{174} \mathrm{Lu}$ Phys. Rev. C80 014304

[447] Dracoulis G D, Kondev F G, Lane G J, Byrne A P, Kibédi T, Ahmad I, Carpenter M P, Freeman S J, Janssens R V F, Hammond N, Lauritsen T, Lister C J, Mukherjee G, Seweryniak D, Chowdhury P, Tandel S and Gramer R 2004 Identification of Yrast High-K Isomers in ${ }^{177} \mathrm{Lu}$ and Characterisation of ${ }^{177 m}$ Lu Phys. Lett. B584 22-30

[448] Kondev F G, Dracoulis G D, Lane G J, Ahmad I, Byrne A P, Carpenter M P, Chowdhury P, Freeman S J, Hammond N, Janssens R V F, Kibédi T, Lauritsen T, Lister C J, Mukherjee G, Seweryniak D and Tandel S K 2004 K-Mixing and Fast Decay of a Seven-quasiparticle Isomer in ${ }^{179}$ Ta Eur. Phys. J. A22 23-7

[449] Palalani N, Lane G J, Dracoulis G D, Kondev F G, Byrne A P, Carpenter M P, Chiara C J, Chowdhury P, Hughes R O, Janssens R V F, Lauritsen T, Lister C J, McCutchan E A, Seweryniak D, Stefanescu I, Watanabe H and Zhu S 2012 Decay of a three-quasiparticle isomer in the neutron-rich nucleus ${ }^{183} \mathrm{Ta} E P J$ Web Conf. 3506004

[450] Lane G J, Dracoulis G D, Byrne A P, Hughes R O, Watanabe H, Kondev F G, Carpenter M P, Chiara C J, Janssens R V F, Lauritsen T, Lister C J, McCutchan E, Seweryniak D, Zhu S, Chowdhury P and Stefanescu I 2009 Decay of a $\mathrm{K}^{\pi}=21 / 2^{-}, 17 \mathrm{~ms}$ isomer in ${ }^{185} \mathrm{Ta}$ Phys. Rev. C80 024321

[451] Reed M W, Lane G J, Dracoulis G D, Byrne A P, Kondev F G, Watanabe H, Hughes R O, N. Palalani, Carpenter M P, Seweryniak D, Zhu S, Janssens R V F, Lister C J, Lauritsen T, Chowdhury P, Jiang W G and Xu F R 2016 Impact of triaxiality on the rotational structure of neutron-rich rhenium isotopes Phys. Lett. B752 311-316

[452] Irnich H, Geissel H, Nolden F, Beckert K, Bosch F, Eickhoff H, Franzke B, Fujita Y, Hausmann M, Jung H C, Klepper O, Kozhuharov C, Kraus G, Magel A, Munzenberg G, Nickel F, Radon T, Reich H, Schlitt B, Schwab W, Steck M, Summerer K, Suzuki T and Wollnik H 1995 Half-Life Measurements of Bare, Mass-Resolved Isomers in a Storage-Cooler Ring Phys. Rev. Lett. 75 4182-5

[453] Litvinov Yu A, Geissel H, Radon T, Attallah F, Audi G, Beckert K, Bosch F, Falch M, Franzke B, Hausmann M, Hellstrom M, Kerscher Th, Klepper O, Kluge H -J, Kozhuharov C, Lobner K E G, Munzenberg G, Nolden F, Novikov Yu N, Quint W, Patyk Z, Reich H, Scheidenberger C, Schlitt B, Steck M, Summerer K, Vermeeren L, Winkler M, Winkler Th and Wollnik H 2005 Mass measurement of cooled neutron-deficient bismuth projectile fragments with time-resolved Schottky mass spectrometry at the FRS-ESR facility Nucl. Phys. A 756 3-38 
[454] Franzke B 1987 The heavy ion storage and cooler ring project ESR at GSI Nucl. Instrum. Meth. Phys. Res. B24/25 18-25

[455] Bosch F, Litinov Yu A and Stöhlker T 2013 Nuclear physics with unstable ions at storage rings Prog. Part. Nucl. Phys. 73 84-140

[456] Bosch F and Litinov Yu A 2013 Mass and lifetime measurements at the experimental storage ring of GSI Int. J. Mass Spectr. 349-350 151-61

[457] Krumbholz K, Schmidt-Ott W -D, Hild T, Kunze V, Meissner F, Wennemann C, Keller H, Kirchner R, Klepper O, Roeckl E, Schardt D and Rykaczewski K 1995 Evidence for the $\mathrm{I}^{\pi}=8^{-}$, $\mathrm{K}=8$ isomeric state in ${ }^{184} \mathrm{Hf} Z$. Phys. A351 11-2

[458] Köster U, Arndt O, Catherall R, Correia J G, Crepieux B, Eichler R, Fernier P, Fraile L M, Hennrich S, Johnston K, Lukic S, Neuhausen J, Nara Singh B S, Pfeiffer B and Siesling E 2007 ISOL beams of hafnium isotopes and isomers Eur. Phys. J. ST 150 293-6

[459] Kristensen L, Jørgensen M, Nielsen O B and Sidenius G 1964 The Decay of $155 \mathrm{~d}{ }^{177} \mathrm{Lu}$ Phys. Lett. B8 5\%-60

[460] Kondev F G, Zhu S, Carpenter M P, Janssens R V F, Ahmad I, Chiara C J, Greene J P, Lauritsen T, Seweryniak D, Lalkovski S and Chowdhury P 2012 M3 and E4 K-forbidden decays of the $\mathrm{K}^{\pi}=23 / 2^{-}$isomer in ${ }^{177} \mathrm{Lu}$ Phys. Rev. C85 027304

[461] Grieser M, Litvinov Yu A, Raabe R, Blaum K, Blumenfeld Y, Butler P A, Wenander F, Woods P J, Aliotta M, Andreyev A, Artemyev A, Atanasov A, Aumann T, Balabanski D, Barzakh A, Batist L, Bernardes A -P, Bernhardt D, Billowes J, Bishop S, Borge M, Borzov I, Bosch F, Boston A J, Brandau C, Catford W, Catherall R, Cederkäll J, Cullen D, Davinson T, Dillmann I, Dimopoulou C, Dracoulis G, Düllmann Ch E, Egelhof P, Estrade A, Fischer D, Flanagan K, Fraile L, Fraser M A, Freeman S J, Geissel H, Gerl J, Greenlees P, Grisenti R E, Habs D, von Hahn R, Hagmann S, Hausmann M, He J J, Heil M, Huyse M, Jenkins D, Jokinen A, Jonson B, Joss D T, Kadi Y, Kalantar-Nayestanaki N, Kay B P, Kiselev O, Kluge H -J, Kowalska M, Kozhuharov C, Kreim S, Kröll T, Kurcewicz J, Labiche M, Lemmon R C, Lestinsky M, Lotay G, Ma X W, Marta M, Meng J, Mücher D, Mukha I, Müller A, Murphy A St J, Neyens G, Nilsson T, Nociforo C, Nörtershäuser W, Page R D, Pasini M, Petridis N, Pietralla N, Pfützner M, Podolyák Zs, Regan P, Reed M W, Reifarth R, Reiter P, Repnow R, Riisager K, Rubio B, Sanjari M S, Savin D W, Scheidenberger C, Schippers S, Schneider D, Schuch R, Schwalm D, Schweikhard L, Shubina D, Siesling E, Simon H, Simpson J, Smith J, Sonnabend K, Steck M, Stora T, Stöhlker T, Sun B, Surzhykov A, Suzaki F, Tarasov O, Trotsenko S, Tu X L, Van Duppen P, Volpe C, Voulot D, Walker P M, Wildner E, Winckler N, Winters D F A, Wolf A, Xu H S, Yakushev A, Yamaguchi T, Yuan Y J, Zhang Y H and Zuber K 2012 Storage ring facility at HIE-ISOLDE - Technical Design Report Eur. Phys. J. ST 207 1-117

[462] Marginean N, Balabanski D L, Bucurescu D, Lalkovski S, Atanasova L, Cata-Danil G, CataDanil I, Daugas J M, Deleanu D, Detistov P, Deyanova G, Filipescu D, Georgiev G, Ghita D, Gladnishki K A, Lozeva R, Glodariu T, Ivascu M, Kisyov S, Mihai C, Marginean R, Negret A, Pascu S, Radulov D, Sava T, Stroe L, Suliman G and Zamfir N V 2010 In-beam measurements of sub-nanosecond nuclear lifetimes with a mixed array of HPGe and $\mathrm{LaBr}_{3}: \mathrm{Ce}$ detectors Eur. Phys. J. A46 329-36

[463] Alharbi T, Regan P H, Marginean N, Podolyák Zs, Roberts O J, Bruce A M, Alkhomashi N, Britton R, Bucurescu D, Deleanu D, Filipescu D, Ghita D, Glodariu T, Mihai C, Mulholland K, Lica R, Marginean R, Nakhostin M, Negret A, Nita C R, Stroe L, Sava T, Townsley C and Zamfir N V 2015 Lifetime of the yrast $I^{\pi}=5^{-}$state and E1 hindrance in the transitional nucleus ${ }^{136}$ Ce Phys. Rev. C91 027302

[464] Zhu S, Kondev F G, Carpenter M P, Ahmad I, Chiara C J, Greene J P, Gurdal G, Janssens R V F, Lalkovski S, Lauritsen T and Seweryniak D 2011 Gamma-ray coincidence and fast-timing measurements using $\operatorname{LaBr}_{3}(\mathrm{Ce})$ detectors and Gammasphere Nucl. Instrum. Meth. Phys. Res. A652 231-233

[465] Régis J -M, Simpson G S, Blanc A, de France G, Jentschel M, Köster U, Mutti P, Paziy V, 
Saed-Samii N, Soldner T, Ur C A, Urban W, Bruce A M, Drouet F, Fraile L M, Ilieva S, Jolie J, Korten W, Kröll T, Lalkovski S, Mach H, Marginean N, Pascovici G, Podolyák Zs, Regan P H, Roberts O J, Smith J F, Townsley C, Vancraeyenest A and Warr N 2014 Germanium-gated $\gamma-\gamma$ fast timing of excited states in fission fragments using the EXILL\& FATIMA spectrometer Nucl. Instrum. Meth. Phys. Res. A 763 210-20

[466] Jolie J, Regis J -M, Wilmsen D, Saed-Samii N, Pfeiffer M, Warr N, Blanc A, Jentschel M, Köster U, Mutti P, Soldner T, Simpson G S, De France G, Urban W, Drouet F, Vancraeyenest A, Bruce A M, Roberts O J, Fraile L M, Paziy V, Ignatov A, Kisyov S, Lalkovski S, Podolyák Zs, Regan P H, Wilson E, Korten W, Ur C A, Lica R and Marginean N 2015 Test of the SO(6) selection rule in ${ }^{196} \mathrm{Pt}$ using cold-neutron capture Nucl. Phys. A934 1-7

[467] Patel Z, Browne F, Bruce A M, Chiga N,Daido R,Nishimura S,Podolyák Zs, Regan P H,Roberts O J,Sakurai H, Söderström P -A,Sumikama Tand Watanabe H 2014 Commissioning of a $\mathrm{LaBr}_{3}(\mathrm{Ce})$ array with EURICA at RIBF RIKEN Accel. Prog. Rep. 4713

[468] Scheidenberger C, Beckert K, Beller P, Bosch F, Brandau C, Boutin D, Chen L, Franzke B, Geissel H, Knobel R, Kozhuharov C, Kurcewicz J, Litvinov S A, Litvinov Yu A, Mazzocco M, Munzenberg G, Nolden F, Plass W R, Steck M, Sun B, Weick H and Winkler M 2006 Isobar separation at FRS-ESR a development towards pure isomeric stored beams Hyperfine Interact. 173 61-6

[469] Kluge H -J 2013 Penning trap mass spectrometry of radionuclides Int. J. Mass Spec. 349-350 26-37

[470] Block M, Bachelet C, Bollen G, Facina M, Folden C M, Guenaut C, Kwiatkowski A A, Morrissey D J, Pang G K, Prinke A, Ringle R, Savory J, Schury P and Schwarz S 2008 Discovery of a Nuclear Isomer in ${ }^{65} \mathrm{Fe}$ with Penning Trap Mass Spectrometry Phys. Rev. Lett. 100132501

[471] Rodriguez Triguero C, Bruce A M, Eronen T, Moore I D, Bowry M, Bacelar A M D, Deo A Y, Elomaa V-V, Gorelov D, Hakala J, Jokinen A, Kankainen A, Karvonen P, Kolhinen V S, Kurpeta J, Malkiewicz T, Mason P J R, Penttila H, Reponen M, Rinta-Antila S, Rissanen J, Saastamoinen A, Simpson G S and Aysto J 2012 Trap-assisted separation of nuclear states for gamma-ray spectroscopy: the example of ${ }^{100} \mathrm{Nb}$ J. Phys. G39 015101

[472] Stanja J, Borgmann Ch, Agramunt J, Algora A, Beck D, Blaum K, Bohm Ch, Breitenfeldt M, Cocolios T E, Fraile L M, Herfurth F, Herlert A, Kowalska M, Kreim S, Lunney D, Manea V, Minaya Ramirez E, Naimi S, Neidherr D, Rosenbusch M, Schweikhard L, Simpson G, Wienholtz F, Wolf R N and Zuber K 2013 Mass spectrometry and decay spectroscopy of isomers across the $\mathrm{Z}=82$ shell closure Phys. Rev. C88 054304

[473] Wolf R N, Wienholtz F, Atanasov D, Beck D, Blaum K, Borgmann Ch, Herfurth F, Kowalska M, Kreim S, Litvinov Yu A, Lunney D, Manea V, Neidherr D, Rosenbusch M, Schweikhard L, Stanja J and Zuber K 2013 ISOLTRAP's multi-reflection time-of-flight mass separator/spectrometer Int. J. Mass Spec. 349-350 123-33

[474] Plass W R, Dickel T and Scheidenberger C 2013 Multiple-reflection time-of-flight mass spectrometry Int. J. Mass Spec. 349-350 134-44

[475] Schury P, Wada M, Ito Y, Naimi S, Sonoda T, Mita H, Takamine A, Okada K, Wollnik H, Chon S, Haba H, Kaji D, Koura H, Miyatake H, Morimoto K, Morita K and Ozawa A 2013 A multi-reflection time-of-flight mass spectrograph for short-lived and super-heavy nuclei Nucl. Instrum. Meth. Phys. Res. B317 537-43

[476] Dickel T, Plass W R, Ayet San Andres S, Ebert J, Geissel H, Haettner E, Hornung C, Miskun I, Pietri S, Purushothaman S, Reiter M P, Rink A -K, Scheidenberger C, Weick H, Dendooven P, Diwisch M, Greiner F, Heie F, Knöbel R, Lippert W, Moore I D, Pohjalainen I, Prochazka A, Ranjan M, Takechi M, Winfield J S and Xu X 2015 First spatial separation of a heavy ion isomeric beam with a multiple-reflection time-of-flight mass spectrometer Phys. Lett. B744 $137-141$

[477] Cheal B and Flanagan K T 2010 Progress in laser spectroscopy at radioactive ion beam facilities J. Phys. G37 113101 
[478] Cheal B, Billowes J, Bissell M L, Blaum K, Charlwood F C, Flanagan K T, Forest D H, Fritzsche S, Geppert Ch, Jokinen A, Kowalska M, Krieger A, Kramer J, Mane E, Moore I D, Neugart R, Neyens G, Nortershauser W, Rajabali M M, Schug M, Stroke H H, Vingerhoets P, Yordanov D $\mathrm{T}$ and Zakova M 2010 Discovery of a long-lived low-lying isomeric state in ${ }^{80} \mathrm{Ga}$ Phys. Rev. C82 051302(R)

[479] Verney D, Tastet B, Kolos K, Le Blanc F, Ibrahim F, Mhamed M C, Cottereau E, Cuong P V, Didierjean F, Duchene G, Essabaa S, Ferraton M, Franchoo S, Khiem L H, Lau C, Le Du J -F, Matea I, Mouginot B, Niikura M, Roussiere B, Stefan I, Testov D and Thomas J -C 2013 Structure of ${ }^{80} \mathrm{Ge}$ revealed by the beta decay of isomeric states in ${ }^{80} \mathrm{Ga}$ : Triaxiality in the vicinity of ${ }^{78} \mathrm{Ni}$ Phys. Rev. C87 054307

[480] Lynch K M, Billowes J, Bissell M L, Budincevic I, Cocolios T E, De Groote R P, De Schepper S, Fedosseev V N, Flanagan K T, Franchoo S, Garcia Ruiz R F, Heylen H, Marsh B A, Neyens G, Procter T J, Rossel R E, Rothe S, Strashnov I, Stroke H H and Wendt K D A 2014 DecayAssisted Laser Spectroscopy of Neutron-Deficient Francium Phys. Rev. X4 011055

[481] Brandau C, Kozhuharov C, Müller A, Bernhardt D, Banas D, Bosch F, Currell F J, Dimopoulou C, Gumberidze A, Hagmann S, Hillenbrand P -M, Heil M, Lestinsky M, Litvinov Yu A, Märtin R, Nolden F, Reuschl R, Sanjari S, Schippers S, Schneider D, Shubina D, Simon H, Spillmann U, Stachura Z, Steck M, Stöhlker Th, Weber G, Wiedeking M, Winckler N and Winters D F A 2013 Probing nuclear properties by resonant atomic collisions between electrons and ions Phys. Scr. T156 014050 